\title{
FLUID FLOW AND HEAT TRANSFER IN A HELIUM GAS SPRING
}

- COMPUTATIONAL FLUID DYNAMICS AND EXPERIMENTS -

\section{Uroš Lekić}




\section{FLUID FLOW AND HEAT TRANSFER IN A HELIUM GAS SPRING \\ - COMPUTATIONAL FLUID DYNAMICS AND EXPERIMENTS -}

Uroš Lekić

November I6, 20I I 
This dissertation has been approved by:

Prof. dr. ir. T.H. van der Meer promoter

Dr. ir. J.B.W. Kok

assistant promoter 


\section{FLUID FLOW AND HEAT TRANSFER IN A HELIUM GAS SPRING}

- COMPUTATIONAL FLUID

DYNAMICS AND EXPERIMENTS -

\section{DISSERTATION}

to obtain

the degree of doctor at the University of Twente, on the authority of the rector magnificus, prof. dr. H. Brinksma,

on account of the decision of the graduation committee, to be publicly defended

on Wednesday the I $6^{\text {th }}$ of November 2OI I at I2:45

by

Uroš Lekić

born on I $7^{\text {th }}$ of April I98I

in Belgrade, Serbia. 


\section{Members of the Promotion Committee:}

Prof. dr. F. Eising

Prof. dr. ir. T.H. van der Meer

Dr. ir. J.B.W. Kok

Prof. dr. ir. H.W.M. Hoeijmakers

Prof. dr. ir. H.J.M. ter Brake

Prof. dr. ir. J. Huetink

Prof. dr. A.E.P. Veldman

Prof. R. Stone
Universiteit Twente, chairman and secretary

Universiteit Twente, promoter

Universiteit Twente, assistant promoter

Universiteit Twente

Universiteit Twente

Universiteit Twente

Rijksuniversiteit Groningen

University of Oxford

This research was carried out under the project name 'Design Rules for Close Tolerance Lubricant Free Piston Compressors', financed by the Technologiestichting STW.

Keywords: Heat Transfer, Fluid Flow, Piston Compressors, Gas Springs, CFD, DNS, Experimental, Thermodynamics.

ISBN 978-90-365-327I-6

Copyright (C) Uroš Lekić, 2OI I

All rights reserved. No parts of this publication may be reproduced, stored in a retrieval system, or transmitted in any form or by any means, without prior written permission of the copyright holder. 


\section{SUMMARY}

The employment of piston compression machines is today extremely wide and versatile. Examples span from common household refrigerators, or internal combustion engines, to highly efficient cryogenic compressors of all sizes and constructions for medical, military and space applications. It is therefore not difficult to grasp the continuing search for the outmost optimization of their efficiency and performance, elimination of any losses and unneeded by-products, and improvements in predictability of their operation.

To further add to these requirements, many state-of-the-art compression technologies move towards lubricant-free solutions in order to maintain the purity of the used operating mediums and so improve the output and availability of their machinery. For this, additional high efforts need to be put in providing and securing narrow tolerance windows of utilized parts and their high stability.

With this thesis and the underlying research, the author attempts to add to the above stated efforts. The work focuses on the fluid flow and heat transfer processes, and the related thermodynamic phenomena occurring in a compressed fluid and at the fluid-wall boundaries of an experimental valveless, unlubricated, one-cylinder piston gas spring.

The presented work is concentrated in three main directions - the experimental work, numerical simulations, and analytical correlating. An experimental machine is newly developed for the needs of this project and equipped with advanced measuring and data acquisition equipment. Experimental data is collected, processed and presented over a range of operating frequencies and two compression ratios. Computational Fluid Dynamics (CFD) models are successfully developed for the numerical work, in order to investigate the applicability of the existing numerical tools for capturing complex processes such as those occurring in the piston compression machines. Full compression 
cycles with no in- and out- flows are modelled. Results are compared and discussed together with the experimentally obtained sets and general thermodynamics principles. Finally, analytical models are investigated and adjusted for several thermodynamic parameters such as the cyclic compression loss, complex Nusselt number, or the thickness of the thermal boundary layers during compression and expansion.

Book in front of you should not be seen as an attempt to present sets of design rules for the piston compression machinery. It is rather a comprehensive summary of the prior existing and newly pursued explorative work in the areas of experimental techniques, numerical modelling and analytical analyses, applicable for capturing the gas-solid heat transfer and fluid-flow processes in gas springs. It should also serve as a useful base for defining additional research efforts, further aiming towards wider industrial applications. 


\section{SAMENVATTING}

Zuigerapparaten worden tegenwoordig voor veel en verschillende toepassingen gebruikt. Voorbeelden hiervan variëren van huishoudelijke koelkasten, verbrandingsmotoren, tot zeer efficiënte cryogene compressoren in een breed scala van formaten en bouw voor medische, militaire en ruimtevaar toepassingen. Het is daarom niet moeilijk te begrijpen dat er een continue zoektocht gaande is naar het verbeteren van hun efficiëntie en prestaties, het minimaliseren van verliezen en ongewenste bijproducten, en het verbeteren van de voorspelbaarheid van hun werking.

Bovenop deze eisen, bewegen vele state-of-the-art compressie technologieën in de richting van smeermiddel vrije oplossingen met het doel de zuiverheid van het gebruikte medium te behouden en op deze wijze de opbrengst en toepasbaarheid van hun apparaten te verhogen. Om dit te bereiken zal aanvullende inspanningen gestopt moeten worden in het verstrekken en behouden van fijne toleranties van de gebruikte onderdelen en verhoging van hun stabiliteit.

Doormiddel van deze thesis en het daar aan voorafgegane onderzoek, probeert de auteur bij te dragen aan de hierboven beschreven inspanningen. Het werk richt zich op de vloeistof stroming en warmte overdracht processen, en de daaraan gerelateerde thermodynamische fenomenen, welke in een samengedrukt fluïdum en aan de fluïdumwand grenzen van een kleploze, ongesmeerde, één-cylinder zuiger gasveer voorkomen.

Het gepresenteerde werk concentreert zich op drie hoofd aspecten - het experimentele werk, numerieke simulaties, en de analytische correlatie. Een experimentele machine is nieuw ontwikkeld voor de behoeften van dit project en uitgerust met geavanceerde meet en data acquisitie apparatuur. Experimentele data is vergaard, verwerkt en gepresenteerd in een gebied van bedrijfsfrequenties en voor twee compressie verhoudingen. Computational 
Fluid Dynamics (CFD) modellen zijn succesvol ontwikkeld voor het numerieke werk, om de toepasbaarheid van bestaande numerieke handvaten voor het beschrijven van complexe processen zoals deze in zuiger compressor apparaten voorkomen te onderzoeken. Volledige compressie cycli zonder in- en uit- stroming zijn gemodelleerd. Resultaten zijn vergeleken en besproken samen met de experimenteel verkregen datasets en algemene thermodynamische princiepen. Tot slot zijn analytische modellen onderzocht en aangepast voor verschillende parameters zoals de cyclische compressie verliezen, complexe Nusselt getallen, of de dikte van de thermische grenslaag tijdens compressie en expansie.

Het boek voor u zou niet gezien moeten worden als een poging om ontwerpregels voor zuiger apparaten op te stellen. Het is meer een omvangrijke samenvatting van het al bestaande en nieuw nagestreefde onderzoekend werk in het gebied van experimentele technieken, numeriek modelleren en analytische analyses, van toepassing op het beschrijven van de gas-wand warmte overdracht en de vloeistof stroming processen in gasveren. Het zou ook moeten dienen als bruikbare basis voor verdere onderzoek inspanningen, welke moet leiden naar bredere industriële toepassing. 


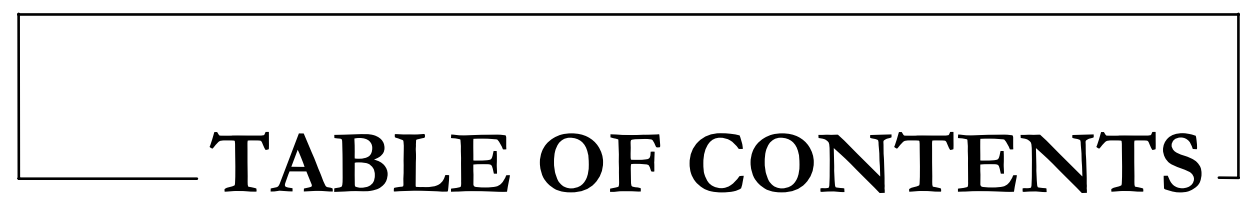

Summary

Samenvatting

Table of Contents $\quad$ IX

I. Introduction I

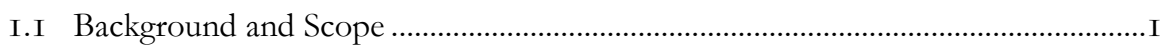

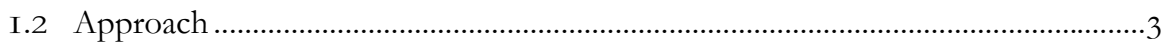

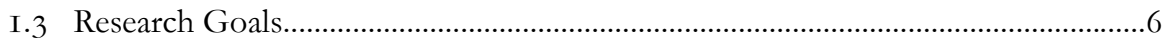

I.4 Thesis Outline ………………....................................................................................

2. Experimental Setup 9

2.I MIT Experimental Setup.........................................................................................

2.2 UT Experimental Setup ....................................................................................... I I

2.2.I Test Rig Design ................................................................................................ I I

2.2.2 Sensing Equipment ..................................................................................... I6

2.2.3 Data Acquisition............................................................................................. I9

2.2.4 Calibration and Data Processing ........................................................................ I9

2.2.5 Executing and the Range of Experiments ..................................................... 20

2.2.6 Measurement Accuracy ...................................................................................... 2I

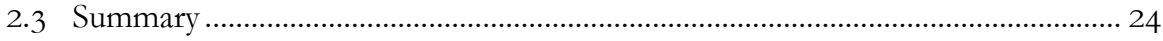

3. CFD Theory 25

3.I Formulation - Governing Equations....................................................................... 25 


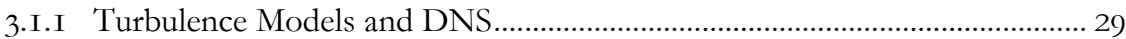

3.2 Numerical Discretization......................................................................................... 30

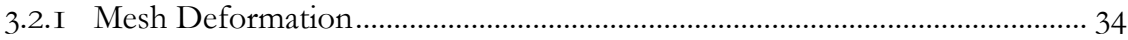

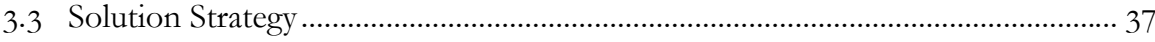

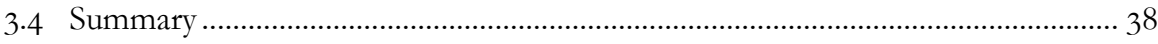

4. Numerical Modelling and Experimental Results 39

4.I Geometries and Spatial Discretization...................................................................... 40

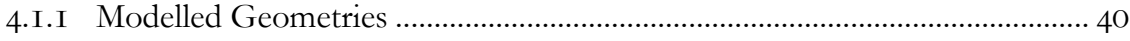

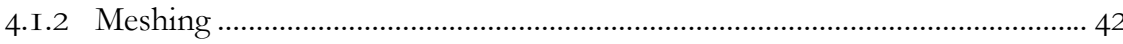

4.2 Temporal Discretization ........................................................................................ 45

4.3 Boundary Conditions and Initial Conditions ........................................................... 46

4.3.I Boundary Conditions .......................................................................................... 47

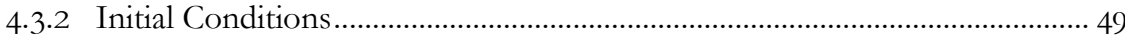

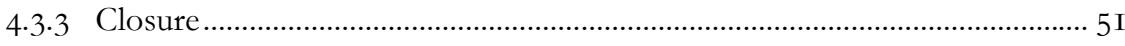

4.4 Estimating Initial Gas Temperature ................................................................... 5I

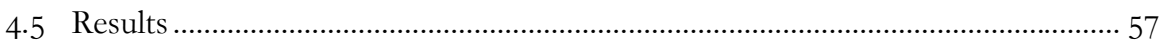

4.5.I Computational Procedure ……………................................................................ 57

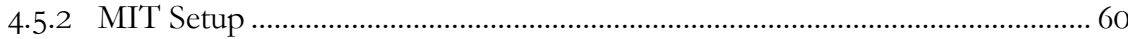

4.5.3 UT Setup - Compression Ratio 2 ……............................................................. 63

4.5.4 UT Setup - Compression Ratio 8 ………...................................................... 73

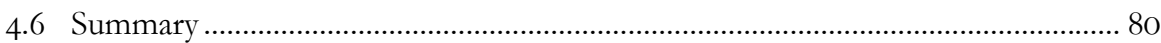

5. Thermodynamics and Fluid Flows $\quad 83$

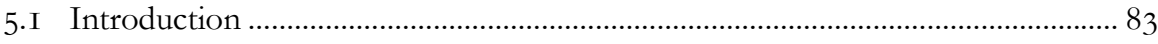

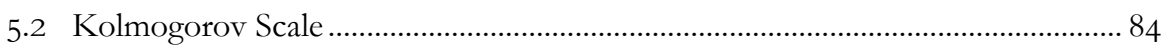

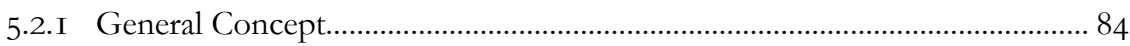

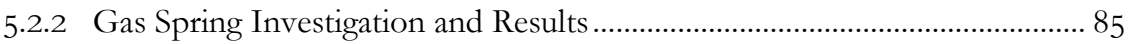

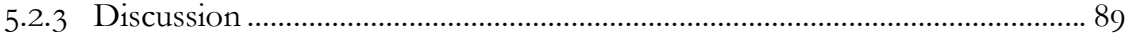

5.3 From Isothermal to Isentropic ......................................................................... 9I

5.3.I Phasing of Physical Properties.............................................................................. 9I

5.3.2 Approaching Ideal Processes............................................................................ 96

5.4 Fluid Flow Patterns and Heat Transfer …………..................................................... 99

5.5 Summary and Conclusions ...................................................................................... IO

6. Analytical Correlations for Gas Springs $\quad 107$

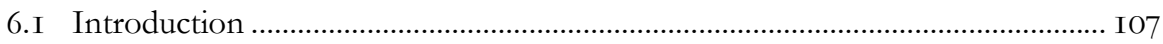


6.2 Cyclic Heat Loss, Pressure Wave Magnitude and Pressure Wave Phase Shift.. I09

6.2.I Background and Analytical Expressions ......................................................... IO9

6.2.2 Numerical Database and Data Processing................................................... I I 2

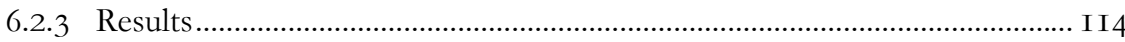

6.2.4 Discussion ...................................................................................................... I I

6.3 Complex Nusselt Number and Instantaneous Heat Transfer............................ I20

6.3.I Formulation............................................................................................... I2I

6.3.2 Analytical Expression .................................................................................. I22

6.3.3 Numerical Data Processing............................................................................... I 24

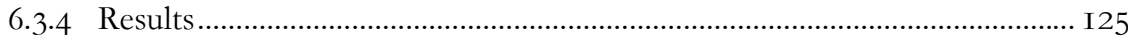

6.3.5 Conclusions .................................................................................................... I29

6.4 Thermal Boundary Layers in Gas Springs......................................................... $\mathrm{I} 30$

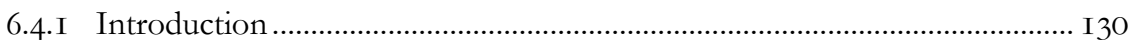

6.4.2 Numerical Results ..................................................................................... $\mathrm{I} 32$

6.4.3 Analytical Correlation and Results .................................................................. I 33

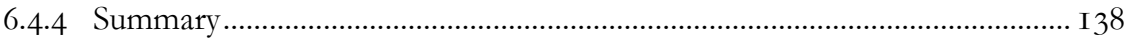

7. Conclusions and Recommendations I39

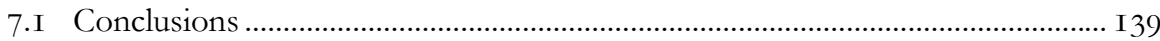

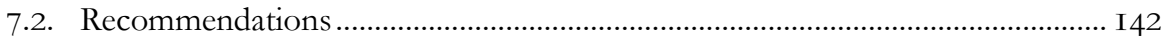

List of References $\quad$ I45

$\begin{array}{ll}\text { Appendices } & \text { I5I }\end{array}$

A List of Partners

B Heat Flux Evaluation Method

C Sensing Equipment and the Assembling Procedure I59

C.I Sensing Equipment............................................................................................... I59

C.2 Assembling Procedure......................................................................................... I6 1

D Increased Charge Pressure Experiments $\quad 165$

$\begin{array}{ll}\text { Final Words } & \text { I69 }\end{array}$ 



\section{CHAPTER INTRODUCTION}

Research that is done at the Laboratory of Thermal Engineering of the University of Twente during the course of a four-year PhD research project is reported in this thesis. This chapter introduces a reader to the motivation and the background for the pursuance of this quest, the research aims and the taken approaches, the project setting and the thesis outline. It summarizes the keywords and topics to be discussed, and aims at informing You of what attempts and achievements can be expected while reading this book.

Enjoy it.

\section{I.I Background and Scope}

Thermal science, as a combined study of thermodynamics, fluid mechanics, heat transfer and combustion, and its appearance in close to every aspect of the modern science and engineering, has been a subject of research in the physics communities for many decades, and continues to be recognized and continuously explored in a search for ever wider understanding and utilization. The purpose of this project is to add to the research methods and approaches in the heat transfer and fluid dynamics considerations, and investigate the physical phenomena related to them and occurring specifically in the reciprocating piston-cylinder machinery.

Employment of the piston compression machines in today's industrial and consumermarket products is very wide and versatile. Examples are the internal combustion engines (automotive, marine and aerospace applications), gas compressors in the cooling devices for domestic and commercial applications (refrigerators), highly efficient miniature cryogenic compressors for military and space applications (Figure I.I), single-stage to 
cascade larger industrial compressors (Figure I.2) and Stirling engines (Figure I.3), medical purposes and superconductivity technology in electronics and so on. In order to improve the reliability and efficiency of these designs, transient thermal and fluid flow phenomena in the compressed medium must be well captured and understood and predicted to the highest reachable extent. Characterization and visualization of the flows inside a compression cylinder is of importance for the design and process optimization and prevention of the instabilities, pressure waves and shocks, characterization of the secondary motion of the moving components, mixing of the compressed fluid et cetera. Accurate modelling of the gas pressures during a compression cycle is significant for optimal dimensioning of the parts, process efficiency evaluation, timing of the intake and exhaust valves, etc. Of certainly no lower importance is the prediction of the heat transfer, its magnitude and the positioning of high flux regions. This allows for correct thermal mapping and design of the compressed space and every exposed component of the machine, the positioning and dimensioning of the cooling paths and avoidance of thermally overstressed areas, as well as understanding of the distortions of the projected geometries of designed parts, especially critical in the high-efficiency machinery (Stirling engines, cryo-industry) where very low contact tolerances are imperative. The well known conventional Nu-Re correlations and its successor models, the steady-state boundary layer theory, and the turbulence closure models for the near-wall region fluid flows are not readily applicable for the transient flow and heat transfer processes in the piston-cylinder constructions, where the gas is undertaking rapid compression and expansion, gas velocities in the compressed spaces significantly fluctuate in time and space during a compression cycle, and where the flow is fully bounded by the surrounding walls.

The problem is for this research simplified to the modelling of piston-cylinder gas springs; a construction with no intake and exhaust valves. This stems from one of the main focuses of the project - the Stirling-type machinery, and the first goal to improve the understanding of the closed-volume related thermal processes. Pursued research also eliminates the presence of oil films (from lubrication) on the heat transfer surfaces and their influence on the named processes, as well as the effects of oil vapours on the properties of the compressed gasses; the motivation for this is discussed in the following paragraphs. In such research setting, a thermodynamic compression problem with ideally clean bounding surfaces and an ideal-gas utilized as the operating compression medium, is posed and investigated. 


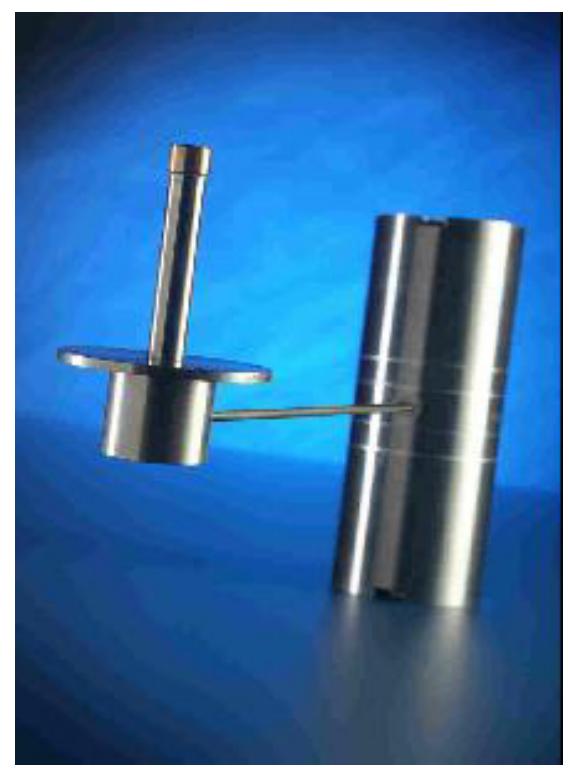

Figure 1.1: Thales miniature linear - dual opposed piston - Stirling cryogenic cooler

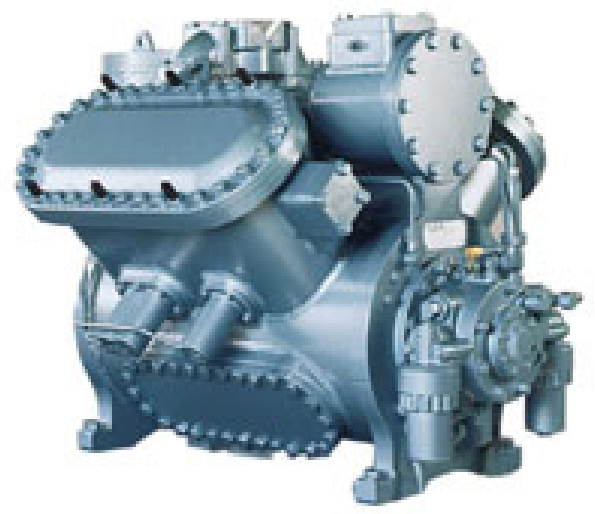

Figure 1.2: Grasso 610 heavy industrial compressor

\section{I.2 Approach}

The work presented here is embedded as a part of a larger research project, a joint effort of the Laboratory of Structural Mechanics, the Laboratory of Surface Technology and Tribology, and the Laboratory of Thermal Engineering at the University of Twente, titled "Design rules for close tolerance and lubricant free piston compressors". In this purview, the ultimate higher goal of the project is to (ideally synchronously) improve the thermomechanical, tribological, and thermal solutions and designs, and assist the performance of the present reciprocating machinery towards the high efficiency, close tolerance, and nonlubricated piston compressor designs. The thermo-mechanical and tribological aspects are in the focus of a parallel research project, done at the Laboratories of Structural Mechanics, and Surface Technology and Tribology, and published as a separate PhD thesis by Pawel Owczarek $[\boldsymbol{I}]$. Thesis in front of you thus concerns the efforts done on the research of thermal phenomena, nonetheless with strong interaction and collaboration with the aforementioned project. The research is also supported and evaluated by a number of prominent industrial partners, named in Appendix A.

Why lubricant free? In most of today's constructions the piston-cylinder contact is made fluid-tight by the piston rings continuously sliding against the cylinder wall, and are 
conventionally lubricated to reduce the frictional forces and wear and cool the contact surfaces. Nevertheless, the lubricants inevitably pollute the working medium; in the opencycle internal combustion engines this is acceptable to reasonable limits (the lubricant vapour or droplets can be combusted and removed), but when it comes to the closed cooling-cycle compression systems (modern refrigeration machinery), any presence of impurities in the thermal carrier is highly undesirable, and strong efforts are put into avoiding it. The conventional ways to achieve the cleanliness of the operating medium are thorough filtering, careful sealing, and shortening of the maintenance intervals. Nevertheless, this also consequentially reduces the operational performance of the concerned machinery and increases the constructional and operating costs. An alternative solution is then to omit the lubrication itself, and in this way eliminate their adverse effects. This option is naturally considered as the most favourable and its aspects and challenges are investigated in the project, this research is part of.

A few words on the 'thermal' research project itself. First of all, it was crucial to fully understand the nature and the extent of problems that are set before it, and this was done by pursuing extensive literature survey on the past and ongoing alike research projects and machinery prior art (this was done at the start of the project, but also naturally continuously throughout the process). After the initial overview and project definition, the approach and research efforts were clustered and pursued in four main work streams:

- The design, machining, assembly and utilization of the experimental setup,

- Numerical modelling,

- Theoretical considerations and observations, and

- Analytical modelling.

More specifically, per work stream:

- The experimental machine is designed as a joint effort with the Laboratory of Structural Mechanics at the University of Twente under the described larger project, and was designed so to also allow for the tribological experiments. The experimental work and results were very important for the validation of any numerical or analytical models, and a lot of attention was given to the construction and selection and installation of the sensing equipment, in order to obtain as wide and diverse as possible range of good-quality experimental data. In addition to the experimental database obtained from the setup developed at the University of Twente, a second validation resource was made available from a prior research done at the Massachusetts Institute of Technology by dr. Alan Abram Kornhauser [2], and these experimental results were used for validation as well. 


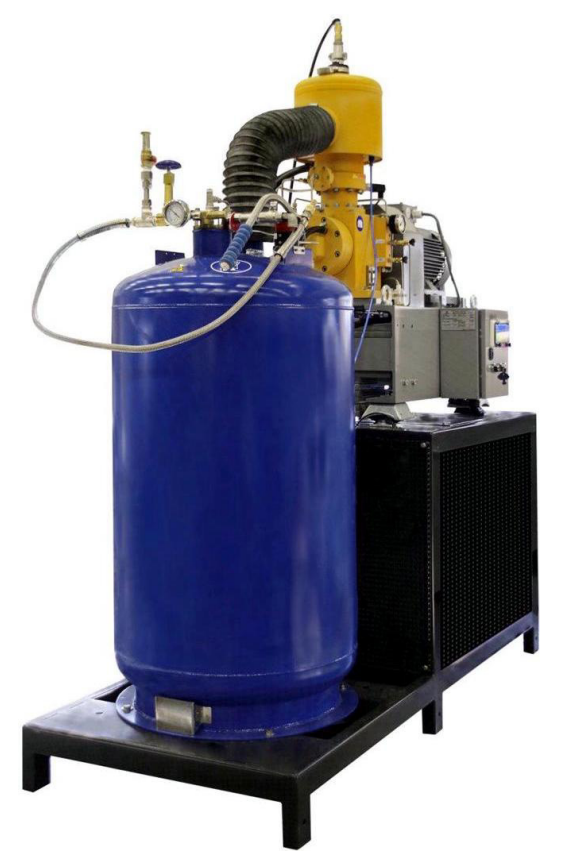

Figure 1.3: The StirLITE liquid nitrogen plant by Stirling Cryogenics

- The numerical modelling (numerical simulating) as a modern, not only research-, but vastly employed and in modern times irreplaceable industrial- design technique, allows for examining the performance of physical systems much prior to the actual physical existence of the represented operational machines. It is thus of very high importance to understand the extent of the achievable physical representation in the simulations, and also put them in the context of the computational costs on one side, and the applicability, performance and reliability of those models on the specific physical problems on the other. For this reason, enormous efforts were put into adjusting and evaluating the results of the created and examined numerical models, and comparing them to the obtained experimental bases.

- Theoretical considerations concentrated on the qualitative analyses, and aimed at gaining insights in the mechanisms of occurring physical phenomena, trying to broaden the search for windows of improvement. Presented general considerations, observations and conclusions are in this way added to the more specific, quantitatively evaluated work pursued in the other three work streams. For this purpose, extensive reading was pursued, on the theory of the thermodynamics, heat transfer processes, fluid mechanics, turbulence, and computational fluid dynamics. 
- Finally, the analytical approach on correlating the phenomena of interest was also investigated. The analytical correlations are often specific and limited to a narrow range of the investigated process conditions while few can be actually applied to more general engineering systems; nevertheless, these can be used as practical tools in obtaining, if not more, estimates for the investigated qualities; often as a first-guess calculator, that can be then followed by the more detailed experimental or numerical evaluations.

\section{I.3 Research Goals}

As described in the previous section, this project is a part of a larger intention to define the design rules for gas compressors utilizing the unlubricated piston-cylinder combination, and with the very small piston-cylinder clearances. The important aspects are to understand the thermal loads and stresses (and the consequential mechanical stresses), and control the tolerances at a (large) temperature range in order to reduce the wear to the necessary, lowest achievable levels. The research that this thesis describes, concentrates thus on this first part, and its goals are accordingly directed to the definition of accurate measurement and prediction methods of the thermal (thermodynamic, fluid mechanic and heat transfer) parameters and the related effects. The line of the project's goals can thus be broken down to the separate targets that are in line with the four work streams discussed in section I.I.2:

- Design of a functioning, modular and adjustable and robust experimental setup, equipped with the state-of-the-art sensing equipment and data logging and post-processing capacity. Validation of the applicable measurement methods, creation of the advanced calibrating, logging and post-processing algorithms;

- Creation of a broad measurement database, comprising of the measured thermal parameters such as the transient values of the gas pressure, gas temperature, cylinder and piston wall surface and in-wall temperatures, and all this for two different compression ratios, different operating frequencies and initial conditions;

- Creation of the robust numerical models using Computational Fluid Dynamics, to accurately capture and predict the above stated physical variables and related properties of interest in any part of a compression process ${ }^{\mathrm{I}}$. This includes proper meshing of the

\footnotetext{
I These models are to be extended in a continued project, with the inclusion of the solid domains and direct evaluation of the thermo-mechanical loads and stresses in the construction, as well the (crucial) distortion of the dimensions related to them. In the second step, the intake and exhaust valves and flows will be added, up to the finally fully functional numerical model, simulating the realtime operation of the compression machines, and directly evaluating all possible thermal concerns.
} 
computational domains, specification of all significant initial and boundary conditions, selection of the appropriate numerical problem solving techniques, as well as the creation of convenient post-processing strategies in order to qualitatively and quantitatively asses the obtained results;

- Investigation of the capabilities of existing analytical relations in predicting the measured and modelled phenomena and, accordingly, their adaptation and derivation of new, improved expressions;

- Increase of the overall thermodynamic knowledge on the subject, summarized and assessed in this thesis, and placement of a base for a continuation of research and further improvements through the follow-up and related projects.

\section{I.4 Thesis Outline}

Chapter I in front of you being the introduction to the thesis, Chapter 2 starts with a short outline of the experimental setup that is built and operated as a part of the mentioned earlier project at the Massachusetts Institute of Technology. Further on, the novel experimental setup designed at the University of Twente is attended in much more detail, explaining the construction specifics, dimensions and materials that are used. An overview of the installed sensing equipment is given, together with the data acquisition system and logging process description, the calibration routine and the measurement accuracy. Also the range of operating conditions is presented.

In Chapter 3 an introduction to the Computational Fluid Dynamics $(C F D)$ package used during the course of the research is given. Care has been taken to present the CFD governing principles and equations, the discretization techniques and the solution-finding methods specific to the ANSYS CFXI I.o code. Deforming meshes and the utilization of DNS approach in place of using the turbulence models are also discussed.

Chapter 4 opens by presenting the numerical models in details, with all the specifications of the simulated domains, meshing and temporal discretization specifics, the operating conditions, numerical accuracy and the computational costs. Further, a method is presented to dynamically estimate the gas temperatures and the correlated mass of the captured gas in a gas spring. This is finally followed by a wide discussion on the measurement and simulation results, starting with the processing of raw data and then moving to the actual results of the simulations. The controlled parameters are the gas pressure and the wall heat flux. The mass transfer from- and to the compressed section of the UT gas spring is further included in the models and also presented here. 
Chapter 5 concentrates on the theoretical considerations, and starts by investigating the conformance of the meshed numerical models to the Kolmogorov scale criterion. In the following subchapter, the observations on the thermodynamic phenomena related to the dynamics of the two typically slow and fast gas spring runs are presented, extended with a discussion on the phasing of gas properties and the similarities to the ideal compression processes. The chapter closes by a discussion on the fluid-flow patterns developing in the compressed closed cylindrical volume, and the forming and disappearance of the flow structures and boundary layers.

Chapter 6 gives an overview of the analytical considerations and expressions, applicable to the investigated geometries and processes. Non-dimensional thermal parameters like the cyclic hysteresis heat loss, pressure wave magnitude and pressure wave phase shift are evaluated, followed by the investigation of the complex Nusselt number, and finally the relations for the boundary layers thicknesses. The existing expressions are discussed and evaluated, and then the analytical and empirical corrections, or new expressions are proposed.

A discussion and conclusions are given at the end of every concerned chapter, while the main points are summarized in Chapter 7 , together with the ideas and recommendations for the continued interest or a follow-up research on the subject. 


\section{CHAPTER}

EXPERIMENTAL SETUP

The experimental machines in the scope of this thesis are two valveless unlubricated gas springs. One setup was built and operated at the Massachusetts Institute of Technology by A.A. Kornhauser, in pursue of his PhD degree and a short overview of it is given here. The second experimental setup is developed at the Laboratory of Thermal Engineering at the University of Twente. Diverse sensing equipment was utilized, and a wide range of experiments was pursued in order to directly measure the parameters in scope. Goal is the creation of the measurement databases for evaluation of performance of the helium gas spring concept, and validation of the theoretic, analytical and numerical models.

This chapter reports on the construction of the experimental setups, used sensing equipment, data acquisition procedures and the data processing, range of performed experiments and the measurement accuracy.

\section{I MIT Experimental Setup}

With courtesy of Dr. Alan Abram Kornhauser from the Massachusetts Institute of Technology (MIT), his extensive work on some of the topics discussed in this thesis on the experimental and analytical aspects of the thermodynamic performance of gas springs, was available very early in this project. The construction of the MIT experimental setup was in base lines very similar to the concept of the gas spring developed at the University of Twente (UT), and this allowed for numerical and theoretical work to be pursued as part 
of this $\mathrm{PhD}$ research much prior to the actual start of the UT experiments. Both experimental setups are one piston-cylinder gas springs, with pistons undergoing reciprocating motion in confined spaces with no inflow and outflow. Both gas springs are operating non-lubricated, omitting the effects of the operating gas contamination and oil films at the bounding solid walls that would affect the thermodynamic processes. Based on this similarity, conclusions drawn on one setup could also be extrapolated to the other geometries and vice-versa, and thus the work based on the experiments done at MIT holds a high value for the outcome of the research presented in this thesis.

For a detailed description of the construction of the MIT experimental setup and the specification of the installed sensing equipment the reader is referred to the $\mathrm{PhD}$ thesis of Kornhauser [2]. A short overview, for the sake of understanding of the work done in this research, is presented here. The MIT experimental setup is a single piston-cylinder, crankshaft driven gas spring without suction/exhaust valves (Figure 2.4). The test section consisting of a piston and cylinder with an adjustable flat head is installed on top of an existing compressor base. The piston top surface is made of brass, while its body is made of Micarta phenolic; the cylinder liner and the cylinder top are made of steel. The pistoncylinder contact is not lubricated, and the piston seal is constructed as a buna-n O-ring inserted between two leather backup rings. The projected radial clearance between the piston body and cylinder was approximately O.IO $\mathrm{mm}$.

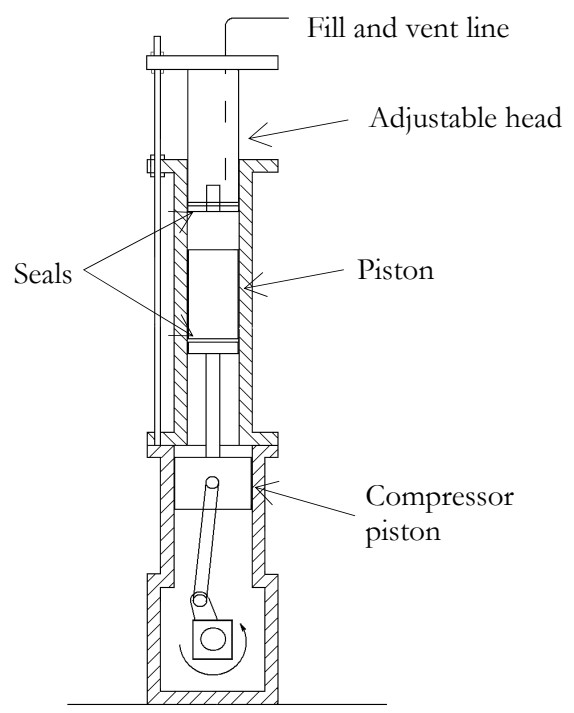

Figure 2.4: MIT one-cylinder experimental setup 
The measured experimental data were collected using a I2-bit analog to digital converter, and the recorded inputs were the pressure signal, the time signal, crank angle encoder pulse and the variable transformer voltage used to pre-set the driving motor speed. The compressed gas volume was then calculated from the crankshaft angle and the driving mechanism geometry. The data sampling was triggered by every eighth pulse of the I2OO counts-per-turn encoder, resulting in 150 data points per revolution. The time signal was provided by an analogue ramp generator. Measurements were recorded once the operating speed and the time-mean cycle pressure became steady, i.e. when the deviation was no more than O.I \% per cycle. At low speeds this criterion had to be relaxed to I \% due to the operating speed variations. Heat flux was indirectly evaluated as a function of pressure, volume and the enclosed mass of the operating fluid on base of thermodynamic relations. The algorithm for the heat flux calculation is presented in Appendix B.

Kornhauser experimented with a range of operating gasses - helium, hydrogen, argon and nitrogen, but only helium will be discussed here as this was the operating medium used in the UT experiments as well. The results for the gas spring with compression ratio 2 and two operating frequencies -2 and IOOO $\mathrm{RPM}^{2}$ are investigated here. Kornhauser also built a compression ratio 8 gas spring, but the measurement results were discussed to be doubtful [2] and were not regarded in this thesis.

\subsection{UT Experimental Setup}

\subsection{Test Rig Design}

The UT experimental setup is also a motored, non-lubricated, one-cylinder, valveless reciprocating gas spring. It is newly designed and developed specifically for the purpose of the research presented here, and, as mentioned in the Introduction, of the research done in a parallel $\mathrm{PhD}$ project at the Applied Mechanics and Surface Engineering and Tribology groups at the University of Twente. In the scope of this parallel project were the tribological aspects of the dry sliding contact and the internal stresses occurring in the parts of high-performance gas compressors. This work is published in the $\mathrm{PhD}$ thesis by Paweł Owczarek $[\boldsymbol{I}]$.

The UT test rig and its schematic representation are shown in Figure 2.5. The compressing section is mounted on the base of a Stirling cryogenerator (type SPC-I), produced and

\footnotetext{
${ }^{2}$ Kornhauser used revolutions per minute - RPM as the operating frequency notation. Since the reference to all UT experiments is more convenient in $[\mathrm{Hz}]$, considerations for the UT experiments and simulations will be in this thesis further presented with [Hz], and for the MIT setup with [RPM] notation.
} 
donated for research by Stirling BV, Netherlands. The compressing piston is in a crosshead arrangement directly coupled to the lower power section via a telescopic rod and the lower power piston, undergoing synchronous semi-sinusoidal motion with it. The lower piston is kept in its original construction and via its own connecting rod connected to a rotating crankshaft. The crankshaft continues on to an internally toothed flywheel and further to an II KW electric motor via an azimuthally rigid and axially compensating coupling. Operation of the electric motor is controlled by a frequency modulator. The whole machine is fixed on a steel frame, which is further bolted to the concrete floor via the vibration-biasing rubber dampers. The contact between the lower piston and lower cylinder is lubricated by the compressor's original oil pump pumping oil through the crankshaft channels. The whole upper section and the contact between the compressing piston and the cylinder liner are dry.

The piston and the adjoining cylinder liner are machined ${ }^{3}$ from dissimilar materials, chosen so to construct a contact with an as low as possible friction coefficient between them. As mentioned, the research on this was done as a part of a parallel project, and the tribological aspects will not be discussed here. The liner is machined from austenitic stainless steel (3I6L), hardened by Kolsterization ${ }^{\circledR}$ process ${ }^{4}$ after machining to very low surface roughness $(\mathrm{Ra}=\mathrm{O} . \mathrm{I} \mu \mathrm{m})$. The piston is made of bronze, as a good counter material for a low sliding friction coefficient contact (0.3 bronze/steel in air [I] $)$, and with similar thermal expansion coefficient $\left(\mathrm{I} 8 \cdot \mathrm{IO}^{-6} \mathrm{~m} / \mathrm{mK}\right.$ for bronze versus $\mathrm{I}^{6} \cdot \mathrm{IO}^{-6} \mathrm{~m} / \mathrm{mK}$ for stainless steel [3]). The cylinder and the piston were designed to create a radial clearance of $30 \mu \mathrm{m}$ at room temperature, but after machining and the Kolsterization of the cylinder the clearance was measured $3 \mu \mathrm{m}$ larger in diameter (Figure $2.6 \mathrm{a}$ ) and b)). The piston is axially aligned with the cylinder and the lower piston by design, and concentricity of the piston and cylinder with the connecting rod. A preventive soft pin is designed in the telescopic connecting rod that would break in the case of seizure between the piston and cylinder inner surface, and protect the lower motoring section from damage.

The compressing part of the machine is created as a modular design, easy adaptable to different compression ratio arrangements. The cylinder head is exchangeable, and by varying its inner height $h$ (Figure $2.6 \mathrm{c}$ )), and by changing only this element, a range of compression ratios can be investigated without altering the remaining of the experimental setup. During the course of this project, compression ratio 2 and 8 heads were constructed

\footnotetext{
3 Entire compressing section, consisting of the compressing piston, compressing cylinder, connecting rod, main frame, and two top frame elements are machined by Ten Heggeler Machinefabriek, Hengelo, Netherlands.

4 Kolsterization process is based on the low temperature $\left(<300{ }^{\circ} \mathrm{C}\right)$ carbon diffusion in the upper layers of stainless steel, enabling the attainment of high surface hardness (around I,O0O - I,200 HVo,05). No post-machining processes are needed after the treatment. Kolsterization is developed by Bodycote Metal Group.
} 


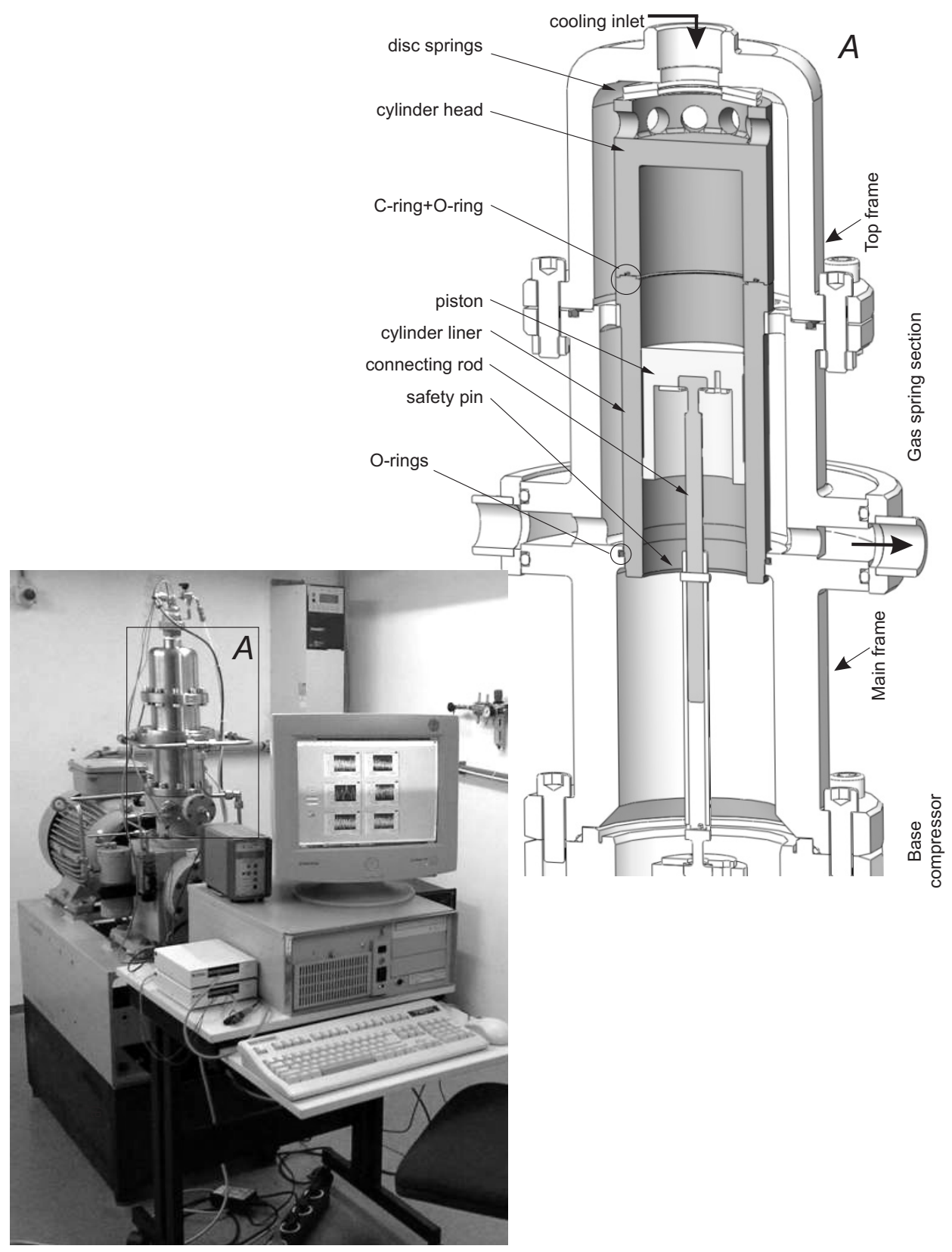

Figure 2.5: UT gas spring experimental rig 


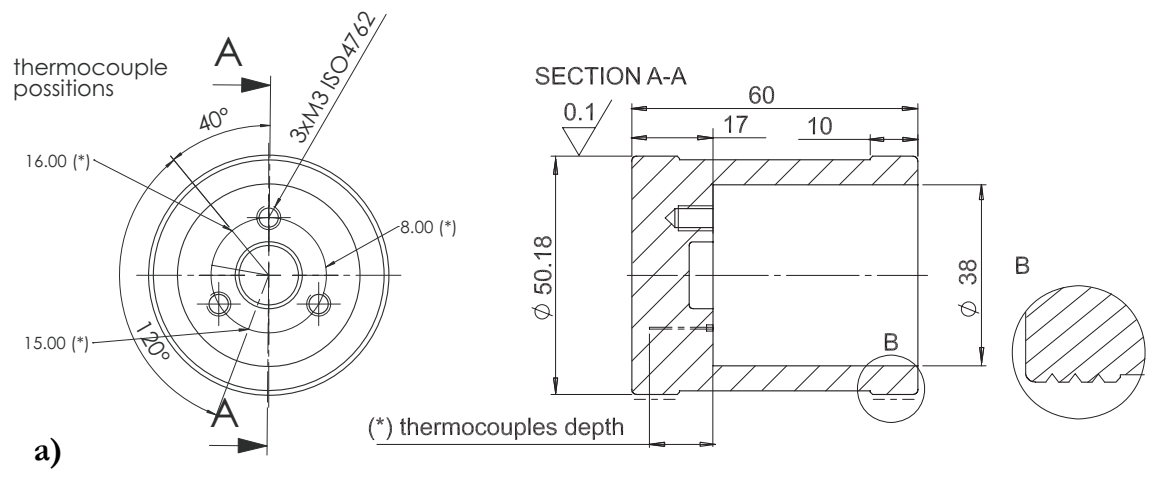

b)
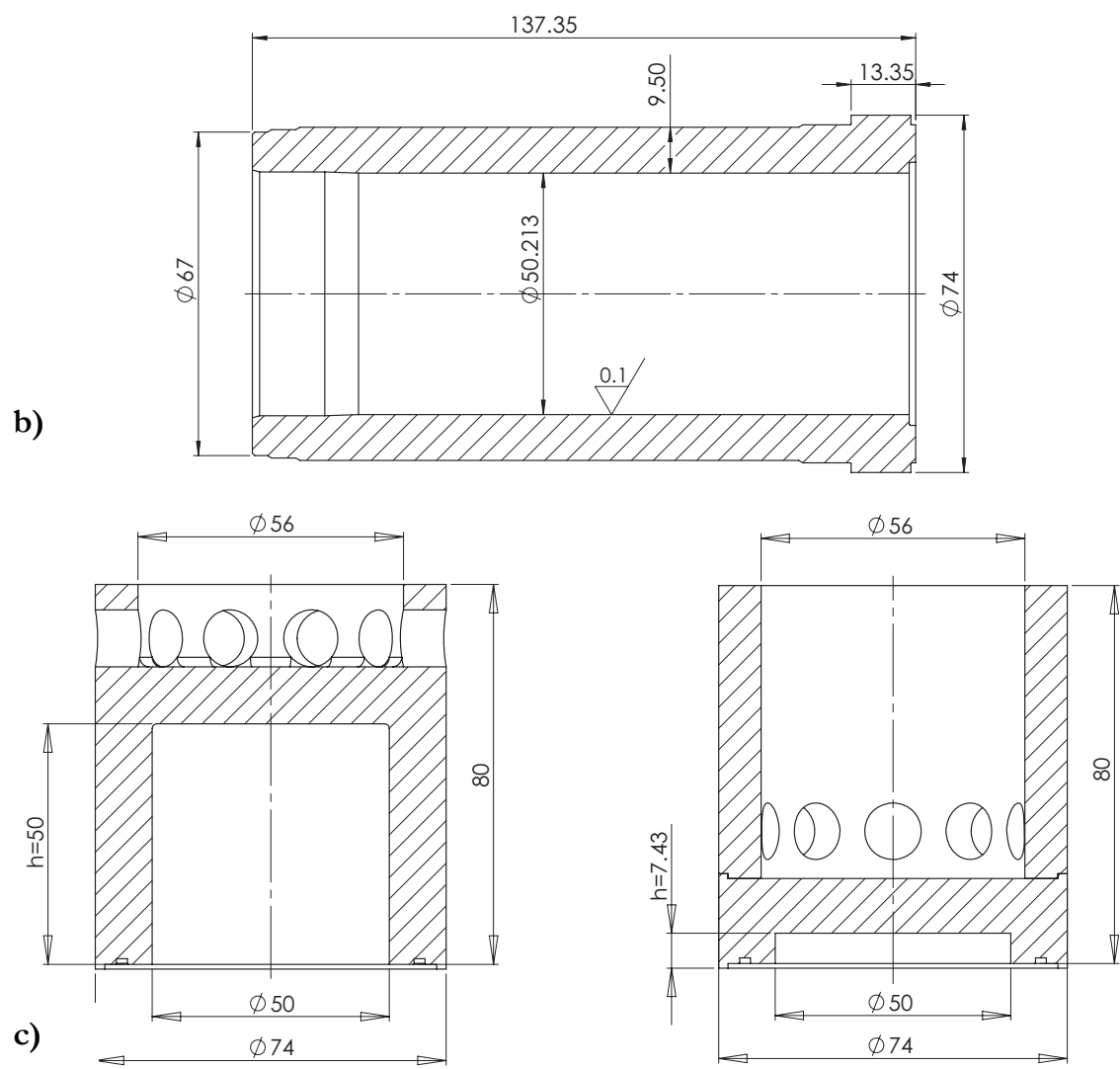

Figure 2.6: UT experimental setup elements: a) piston, b) cylinder liner and c) cylinder head, leftcompression ratio 2, and right - compression ratio 8. Dimensions are expressed in [mm] 


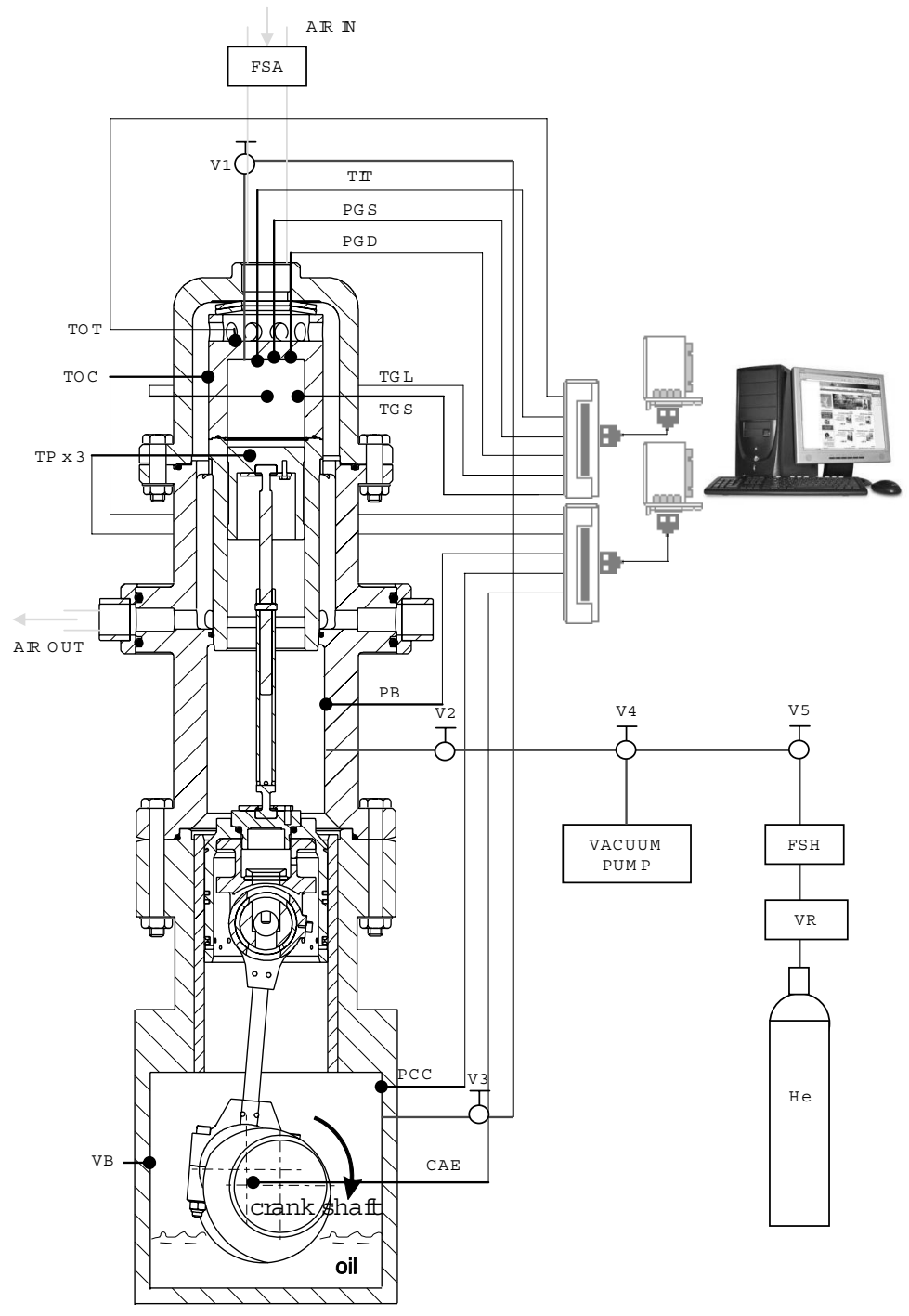

Figure 2.7: Cross-section of the UT gas spring and the schematic diagram of the installation. Legend: FS $A$ - air filtering section, $V_{1}-V_{3}, V_{5}-$ stop valves, $V_{4}-3$-way valve, TIT - inner top thermocouple, TOT - outer top thermocouple, TOC - outer cylinder liner thermocouple, TP - piston thermocouples, PGS, PGD - dynamic gas pressure transducers, TGL, TGS - gas thermocouples long and short, $P B$ - buffer pressure transducer, PCC - crank-case pressure transducer, CAE - crank angle encoder, FSH - helium filtering section, VB - bleed valve and manometer 
and machined from copper (En I360I). The piston stroke (both lower and compressing piston) is determined by the geometry of the lower Stirling driving mechanism and equals $52 \mathrm{~mm}$. Having the crankshaft connecting rod of finite length, the piston motion is not perfectly sinusoidal, and the mid-stroke was reached at $95{ }^{\circ} \mathrm{CA}$.

The operating gas (helium) is fed to the compression section by a gas supply line (Figure 2.7), and distributed by a series of reduction and stop valves, branching in three separated lines to supply the gas to the volumes below and under the compression piston, and the crankshaft housing. The operating gas is pre-filtered by 3 serially placed filters, consisting of one I $\mu \mathrm{m}$ pre-filtering element, one O.OI $\mu \mathrm{m}$ element and an active coal element. The charge pressure is controlled by a reduction valve and a precision manometer mounted on the supply line, and the pressure transducers in the operating section. Helium filling procedure is explained in Appendix C

\subsubsection{Sensing Equipment}

A range of sensing instrumentation is installed in order to capture most of the properties of interest. The compressed section is equipped with two pressure transducers - one static and one dynamic, two miniature gas thermocouples - one close to the cylinder wall and one perturbing to the centre of the compressed gas, one custom designed heat flux sensor with an inner cylinder top surface thermocouple and an in-wall thermocouple, one outer cylinder wall surface thermocouple and one outer cylinder top surface thermocouple. The piston temperature is measured with three thermocouples installed in its body on depths of I mm, $2 \mathrm{~mm}$ and $9 \mathrm{~mm}$ from its surface exposed to the compressed gas. Buffer space pressure and pressure in the crank case are also measured with precision pressure transducers. Finally the position of the piston is sampled with a crank angle encoder with 360 pulses per revolution. Specifications for all measuring equipment 5 is also given in Appendix C.

\footnotetext{
5 Not all installed sensing equipment was actually actively utilized up to the end of this project. Compressed space and buffer space pressure, gas temperature measurements and the crank angle encoder pulse were used in validation of the numerical work presented in Chapter 4. Inner cylinder top surface, outer cylinder surface and the piston temperature (closest to the compressing surface) measurements were used for setting initial and boundary conditions for the mentioned numerical simulations. In-wall cylinder top temperature measurement and outer surface cylinder top measurement were merely the steady-state indicators, although initially installed to measure the heat transfer from the compressed section. Pressure measurements of the crankcase volume were useful only during the initial helium charging process. Finally, the other two piston body thermocouples were installed to observe the temperature oscillations penetration depth and heat transfer in the piston, and estimate the thermal expansion of the piston. This was recorded but not analyzed, and together with all the above mentioned, serves as a potential source for future analyses.
} 


\section{Pressure Transducers}

The compressed gas pressure was initially measured only with a precision piezoelectric pressure transducer, selected for its very compact design and robustness. Coupled with the programmable charge amplifier it was scaled to measure the O-IO bar range with extremely low signal noise (typically less than $0.5 \%$ of the measured amplitude). Nevertheless, an inherent property of the piezoelectric sensing equipment is the charge drift which disables its usage for absolute measurements. The charge amplifier was equipped with a drift compensation circuit, but nonetheless it gave erroneous results. For this reason a second, displacement pressure transducer was built-in to measure the absolute pressure of the compressed gas and help level the dynamic pressure measurements. This sensor produced a much higher signal noise (up to IO \% of the recorded signal magnitude - section 2.2.6), but with the sample averaging routine presented later in this chapter the pressure trend and amplitude were still well captured.

The buffer and crankcase pressure were measured with conventional diaphragm pressure transducers with increased accuracy. All sensors that were installed had to have metalshielded hermetically sealed sensing elements for protection of the sensing circuits from helium perturbing to the sensor and damaging the silicone membranes, or introducing errors in measurements by means of equalizing internal pressures in front of and behind the membrane.

\section{Thermocouples}

A wide range of temperature sensing equipment was tested and installed. Attempts to measure the instantaneous gas temperature were pursued by installing custom miniature thermocouples with a sensing end in the order of $<8 \mu \mathrm{m}$ in size and I ms time response according to the producer ${ }^{6}$. Still, performance of even such a small sensing element is by design dependent on the flow rate of the measured medium, and in the geometry as investigated here where the gas comes to stand-still in parts of the cycle, their applicability to direct instantaneous temperature measurement at high operation rates was found to be inadequate. Nevertheless, a new method to estimate the cycle gas temperature from the recorded signals was developed and presented in subchapter 4.4. Two thermocouples were installed: one $6 \mathrm{~mm}$ from the wall in the gas (the shortest machineable length for the sensor), and the second in the centre of the compressed volume (25 $\mathrm{mm}$ in length).

The surface temperature of the cylinder top on the side exposed to the gas was measured with an eroding type thermocouple. The sensor is constructed by placing two isolated thin

\footnotetext{
${ }^{6}$ When calculated, the thermal mass of the sensing end for the used Chromel-Constantan E-type thermocouples amounts to $\sim \mathrm{Ie}^{-6} \mathrm{~J} / \mathrm{K}$, and the time constant, for the maximum piston velocity occurring in the examined experiments and developed flow, is $\sim \mathrm{I} .8 \mathrm{~ms}$. More on this is presented in subchapter 4.4 .
} 


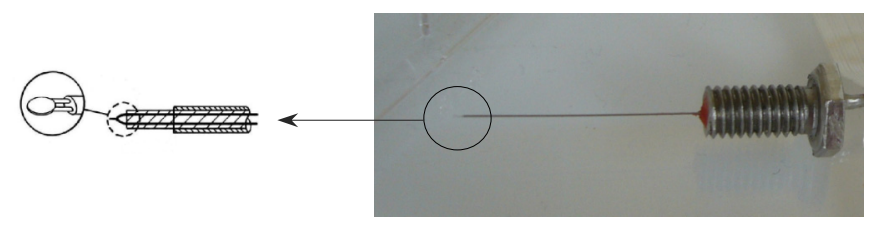

Figure 2.8: Miniature gas thermocouples; aluminium shield tube $0.2 \mathrm{~mm}$ outer diameter, micro disc junction $<7.6 e^{-3} \mathrm{~mm}$
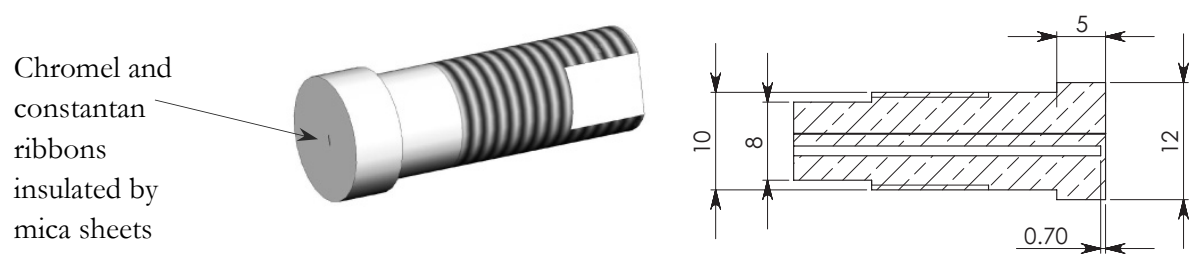

Figure 2.9: Inner cylinder top surface thermocouple. Copper body and eroded surface hot junction; in-wall thermocouple is displaced $0.7 \mathrm{~mm}$ from the exposed surface

thermocouple material ribbons in the bulk sensor body and fine-sanding the sensing surface to form the microscopic thermocouple junctions, and give time response of $8 \mu \mathrm{s}$ according to the supplier [4], and from I to $20.8 \mu$ s as reported in literature [5], [6]. The sensor body material was chosen to match the cylinder head in which it was installed (copper), so as not to disturb the heat flows in the material. The exposed sensing surface with the sanded thermocouple junction is mounted flush with the head surface. A back thermocouple with the time response of $70 \mathrm{~ms}$ is displaced from surface and mounted in the sensor body to directly measure the wall heat flux. The procedure for evaluating the heat flux directly from the two thermocouples readings was not developed before the end of this project, and the back thermocouple signal was not examined in that context but only as an independent in-body temperature measurement.

Surface thermocouples installed on the outer cylinder wall and cylinder top surfaces were off-the-shelf self adhesive thermocouples. Infra-red temperature sensing of the outer wall surface was done with a short-range pyrometer, but this method did not bring any advantages compared to the thermocouple usage and was abandoned.

The piston temperature was measured with three shielded thin thermocouples $(0.5 \mathrm{~mm}$ in diameter), placed on two depths close to the compressing piston surface and one centrally in its body ( $\mathrm{I} \mathrm{mm}, 2 \mathrm{~mm}$ and $9 \mathrm{~mm}$ from the compressing surface, Figure 2.6a). The thermocouples are also commercially available, and are immersed in the $0.75 \mathrm{~mm}$ electrically eroded sleeves and sealed with highly thermally conductive resin. 


\section{Crank Angle Encoder}

Rotationally rigid coupling between the compression machine crankshaft and the electric motor enabled the mounting of the crank angle encoder on the motor shaft end. The encoder produces 360 digital pulses per revolution (together with the 'zero' pulse), and indirectly determines the piston position and thus the instantaneous compressed volume. Matlab procedure was created to translate the pulses to the bottom-dead-centre $(B D C)$ position and compression cycle increments.

\subsubsection{Data Acquisition}

The data acquisition system is composed of two National Instruments PCI cards installed in a personal computer configuration selected for the most efficient sampling ${ }^{7}$, and two National Instruments terminal blocks as connection ports to the sensing equipment. Overall 32 single ended/I6 differential analogue input channels with input range O-IO V and 24 digital input/output channels are available. One PCI card has very high-accuracy and I8-bit resolution for accurate low-voltage measurement, typically met with thermocouple signals. The maximum sampling rate is $625 \mathrm{kS} / \mathrm{s}$ in the single channel mode, or $500 \mathrm{kS} / \mathrm{s}$ aggregate in the multichannel mode (multichannel mode used in the experiments). The second PCI card is a I6-bit high-speed card used for sampling of the pressure and encoder signals, with the capacity of up to I.25 MS/s for single, i.e. I.OO MS/s aggregate for multichannel sampling. The internal clocks of the cards are synchronised by an RTSI cable and programmed by the software interface. The sensor wires were carefully guided from the setup to the shielded terminal screw blocks. Thermocouple wires are shielded with aluminium foil and the computer casing was grounded for noise elimination.

Data is stored by the custom written LabView scripts. Data is sampled at rates from $5 \mathrm{kHz}$ for $2 \mathrm{~Hz}$ operating frequency to $36 \mathrm{kHz}$ for $25 \mathrm{~Hz}$ runs, using all 32 input channels. This allowed for a resolution of at least I440 samples/revolution for the critical - fastest run. Since the PCI cards were synchronized, the upper sampling limit for the system was determined by the card with a lower data throughput.

\subsubsection{Calibration and Data Processing}

All the sensors were carefully calibrated prior to installation. All thermocouples that are used in the setup were calibrated at once. Thermocouples were physically grouped and jointly immersed in a mixed water bath, and calibrated with a high-precision mercury

\footnotetext{
7 Intel Core2 Duo, $2.33 \mathrm{GHz}$, wide front bus for high data stream through-put: I.333MHz FSB, 4MB L2 cache.
} 
thermometer over the range of temperatures from $\mathrm{O}$ to $\mathrm{IOO}{ }^{\circ} \mathrm{C}$. Micro-voltage output was recorded using the same marked channels as in the experiments, and then the calibration curves were created for every unique sensor.

The same procedure was repeated for the pressure transducers. The high precision pressure sensor (0.04 \% FS BSL) installed in the buffer space was sent to the manufacturer for calibration, and then the other three transducers were calibrated relative to this signal. As with the thermocouples, sampling was done synchronously, on a pressurized helium line using a custom made connecting junction. Transducers were calibrated from the best achievable vacuum (using a molecular vacuum pump to the minimum of $\sim 40$ mbar) to the upper range of Io bar.

All measurement data was sampled and recorded raw, as the direct analogue and digital (from the angle encoder) output of the sensing equipment. This was done for the reduction of the processing load on the personal computer and the higher sampling rates attainment. Recorded signals were then post-processed using the calibration curves obtained earlier, as explained.

\subsubsection{Executing and the Range of Experiments}

Helium was the only investigated operating gas. Two compression ratio gas springs were constructed -2 and 8 . For every compression ratio, a gas spring was operated at a range of operating frequencies $-2,5, \mathrm{IO}, \mathrm{I} 5,2 \mathrm{O}$ and $25 \mathrm{~Hz}$. A range of initial charge pressures was also investigated for compression ratio 2, and this was set at I.5, 3, 5 and 8 bar. For the compression ratio 8 gas spring, experiments were done only with the I.5 bar charge pressure. As will be presented in the last paragraph of this section and Chapter 4, because of the inherent leakage past the compressing piston and the experimental procedure, the actual initial pressures in the representative compression cycles were significantly lower.

After the vacuumizing and purging of a gas spring prior to the experiment ${ }^{8}$, the gas spring was charged to the set pressure with the piston in the BDC position and left for a short period of time ( $\mathrm{I} 5$ minutes) for pressures in all the volumes in the setup to equalize. After that, the gas spring operation was started at the lowest frequency $-2 \mathrm{~Hz}$ and ran until the controlled parameters - gas pressure, gas temperature and temperature of the walls in the compression section would stabilize within $0.5 \%$ of the measured amplitude (this was assessed from the real-time output viewer programmed in the sampling code, and the stabilization routine typically took $\sim 50$ cycles). A batch of 20 operating cycles was

\footnotetext{
${ }^{8}$ The air evacuating and gas filling procedure is described in Appendix C
} 
recorded then 9 . After data sampling, the machine operation was directly continued with the frequency increased to the next set-point ( $5 \mathrm{~Hz}$, IO $\mathrm{Hz}$, etc.). The same cycle stabilization and sampling procedure were repeated until the maximal $-25 \mathrm{~Hz}$ operation was recorded. The machine was then stopped and measurement data post-processed.

As mentioned, the radial gap between the piston and the cylinder is the inherent shortcoming of the piston design as presented here and used in this research ${ }^{\mathrm{Io}}$. This gap allows for gas leakage past the piston (evaluated in Chapter 4), that is limited per one cycle but still relevant over a large number of cycles that were typically run from the start of operation to the actual data recording. This caused the cycle-mean pressure equalization on two sides of the compressing piston, and the lowering of the cycle BDC pressure. This translated the I.5, 3, 5 and 8 bar charge pressures to I.06, I.87, 2.83 and 3.94 bar BDC pressures respectively, for the steady-state, compression ratio $2,2 \mathrm{~Hz}$ runs. With the higher operating frequencies, pressure amplitudes were also higher, and the BDC pressures respectively lower. Exact BDC pressures, as initial conditions for all the simulations for UT experiments are summed in the tables presented at the beginning of every respective section in Chapter 4 .

\subsubsection{Measurement Accuracy}

Measuring a thermocouple signal in the $\mathrm{mV}$ range, pressure transducer signal amplification (or performing any kind of precision measurement) inherently comes with a particular uncertainty and the ever-present measured signal noise. In this research these uncertainties were tackled by several countermeasures. Sensors were calibrated by the above described extensive calibration procedures, whereas eliminating the raw signal noise showed to be a more tedious task. All the electronic equipment in the experimental setup was grounded, the wires were shielded from the surrounding magnetic fields and all the thermocouples were connected to the terminal blocks in differential arrangement. Some residual noise was still inevitably present in the sampled signals, although, for the considerations pursued in the research presented here, they could be regarded acceptable, as shown in the next paragraph.

Quantitative evaluation was made on the extent of this noise, and the sample variance of the raw measurements for all the installed sensors was computed. The results are displayed

\footnotetext{
9 This was done in intention to perform cycle-averaging of the data over a number of cycles. Since it was possible to record $\mathrm{I} 440$ to 2500 samples per cycle for the whole operating range, this was not necessary, and for the desired IOO measurements per revolution, data was averaged over a (respective) window size, in running batch windows over one cycle. This averaging procedure proved to be very effective, as shown in 2.2.6.

Io Alternative piston designs that overcome this problem are considered in (Owczarek [ $\boldsymbol{I}]$ ).
} 

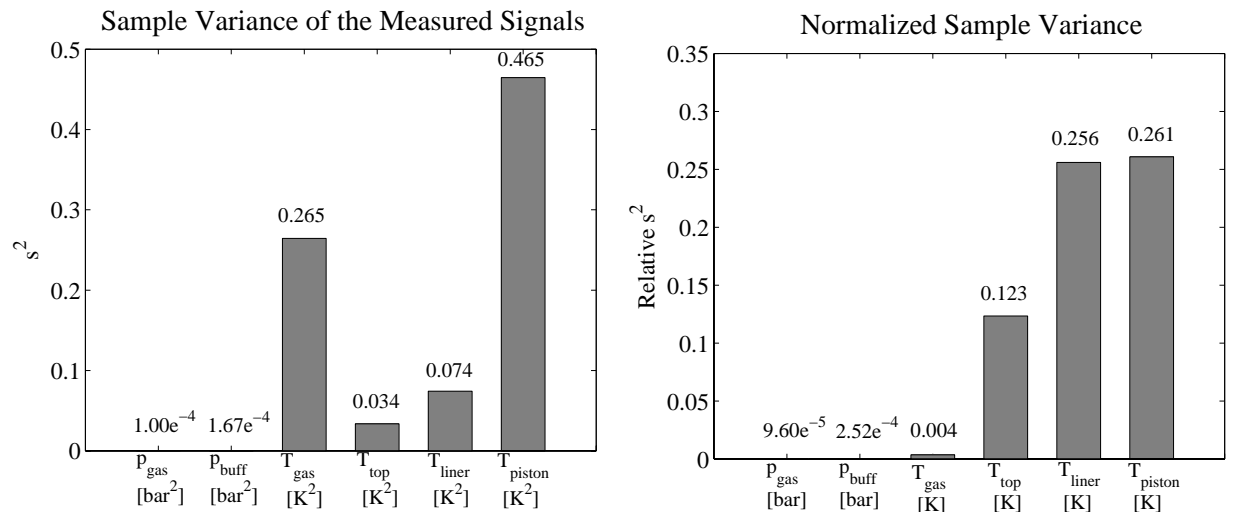

Figure 2.10: Sample variance of the sensor signals, standard variance (left) and normalized with the magnitude of the respective signal (right)
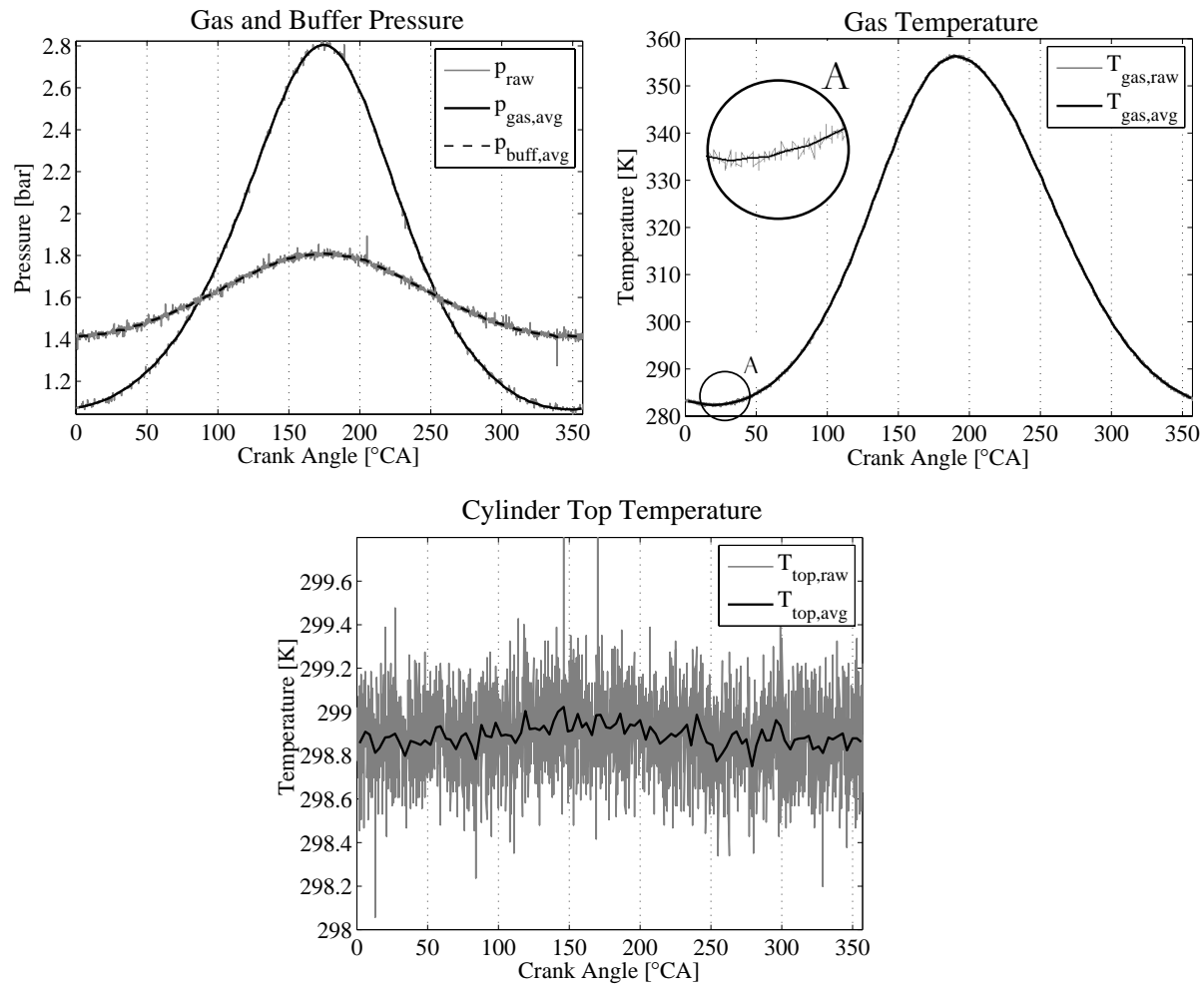

Figure 2.11: Raw and span-averaged measurement signals for the compressed gas and buffer pressure, gas temperature and the inner surface cylinder top temperature 
in Figure 2.IO, and for the scope of this research show reasonable results. Sample variance is normalized with respect to the standard Bessel's correction [7]:

$$
s_{j}^{2}=\frac{1}{n-1} \sum_{i=1}^{n}\left(x_{i}-\bar{x}_{J}\right)^{2},
$$

for $j^{\text {th }}$ batch of samples, each consisting of $n$ samples and evaluated on the span-sample mean $\overline{x_{J}}$. Effectively, it is the normalized square root of the standard deviation of the sampled signal. Figure 2.IO right shows the variance normalized with the magnitude of the respective averaged signal ( $\min$ to $\max$ ), and thus indicates its relevance to a measurement. Since the amplitudes of the cylinder top, cylinder liner and piston temperature were very small, this ratio shows a very significant relative signal noise. Nevertheless, these measurements were included in the numerical simulations as their mean (and constant over a compression cycle) values, and thus do not require additional attention here. For the qualitative surface temperature considerations, and possibly direct heat flux evaluation though, extra care will needs to be taken.

Although the signal noise for the key properties - gas pressure and gas temperature was small, the installed equipment allowed for sampling rates significantly higher than the inverse of the respective physical time-scales, and the averaging of the measured data over a span (window size) was possible. The arbitrarily chosen desired resolution of the resulting signals was 100 sample points per cycle, and this determined the window sizes ranging from $\mathrm{I} 44$ for the fastest, $25 \mathrm{~Hz}$ runs to 250 samples per one 'averaged' point for the slowest $2 \mathrm{~Hz}$ runs. The effects of window-averaging are visible on Figure 2.I I, where the smoothing of e.g. the cylinder top inner surface temperature is very obvious. The noise amplitude is here reduced by $80 \%$ - from $\sim 0.5 \mathrm{~K}$ to O.I $\mathrm{K}$ The window averaging procedure proved to be even more effective for the gas pressure and gas temperature noise, where it was basically completely eliminated and the curves smoothened out.

Since all sensors of each group (surface and piston thermocouples, pressure transducers) were calibrated simultaneously, from these measurements the absolute accuracy of the sensors could also be evaluated, after averaging and calibration corrections. For the surface thermocouples the highest absolute error was $0.3 \mathrm{~K}$ and for the piston thermocouples 0.43 $\mathrm{K}$. together with the residual measurement noise reported above. After correcting for calibration the errors of pressure transducers were practically eliminated and the inaccuracy of the pressure measurements was in the order of the residual noise.

The time synchronization of the two PCI cards was tested by connecting the angle encoder pulse and cycle-zero signals, each on one of the PCI cards and investigating the recorded signal on the time scale (obtained from the computer's internal clock, as in the 
experiments). Findings have shown a very satisfactory synchronization accuracy, being that the observed recorded signals could be mapped on another within $0.2 \mathrm{e}^{-7}$ seconds.

\subsection{Summary}

Experimental research is one of the four modules pursued in this project. Creation of experimental databases serves as an important source for theoretical considerations and validation of the modelling work. Two experimental setups have been regarded in this research for this purpose, and explained in this chapter in their key points.

Experimental work on one of the gas springs, built and operated at the Massachusetts Institute of Technology, was done by A.A.Kornhauser [2] over two decades ago. Nevertheless, due to the similarity of the utilized experimental setup to the concept of the machine built at the University of Twente, the measurement data obtained from these experiments were a useful base for the analytical and numerical work long before the inhouse experiments could start. The MIT experimental gas spring is a valveless unlubricated piston compressor, equipped with a pressure sensor and a crank-angle encoder. Compression ratio 2 experimental data were analyzed. A short overview of this setup is given in subchapter 2.I.

The gas spring developed at the University of Twente is a new setup designed to allow for a wide range of operating conditions and very diverse experimental measurements. A frequency controller and the modular construction of the compressing section allow for a wide range of operating conditions (arbitrary operation frequencies, compression ratios and charge pressures). Here are investigated compression ratios 2 and 8 , and a range of operating frequencies from 2 to $25 \mathrm{~Hz}$. Diverse sensing instrumentation is installed, including four pressure transducers, two gas thermocouples, overall seven surface and inwall thermocouples and a crank-angle encoder. The data acquisition system is composed of two PCI cards installed in a personal computer and two devoted terminal blocks. Maximum sampling rate is $500 \mathrm{kS} / \mathrm{s}$ aggregate for the synchronized sampling of measurement data on 32 input channels.

The measurement data is recorded when the steady-state operation of the machine is ensured, at sampling rates from 5 to $36 \mathrm{kHz}$ ( 2 to $25 \mathrm{~Hz}$ operation frequency), postprocessed for respective calibrations and span-averaged for noise elimination. Effectiveness of the averaging procedure is demonstrated. 


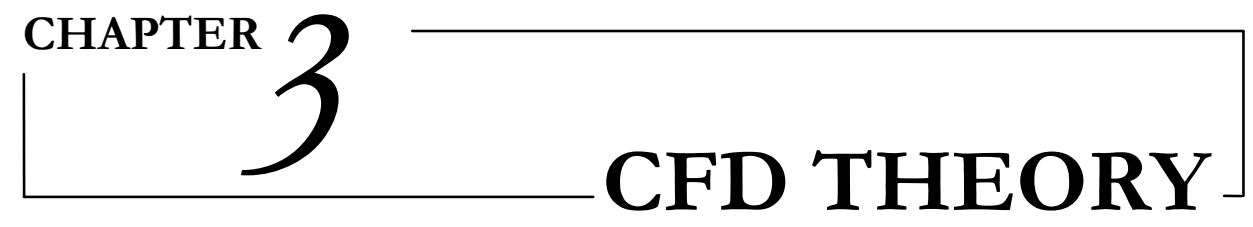

Computational Fluid Dynamics - abbreviated CFD, is a modern discipline developed in the recent scientific history as a new approach in the study of dynamics of fluids. With the vast underlying fluid dynamics theory and the complementing experiments, it extends the broadness, applicability, efficiency and the reach of the analysis and fluid dynamics problem solutions. It serves as a numerical closure to physical principles and laws, and with today's easily available and ample computing power, presents an established method of performing numerical experiments on the industrial designs of interest.

Many books have been written on this topic and it would be impossible to embosom all the equations, solution methods, analytical aspects and stability and error analyses principles in one chapter. For that reason, only the main aspects of the numerical modelling performed along the scope of this thesis, from a user's perspective, are presented here for a general physical and mathematical understanding of the performed simulations. The goal is to provide the reader with a frame for qualitative understanding of the presented results, and a reference base for a continued interest.

\section{I Formulation - Governing Equations}

Navier-Stokes equations were derived in the early nineteenth century and have been the centre point of the fluid dynamics considerations ever since. Unlike algebraic equations, they do not explicitly establish relations among the variables of interest (density, pressure, temperature, velocity), but rather describe relations of their rates of change. Navier-Stokes equations dictate velocity, not position; their solution is a velocity (flow) field, at a given point in space and time, and from it, the rest of the quantities of interest may be derived. 
Describing the general single-phase fluid, under appropriate circumstances, these equations can be somewhat simplified: one can include incompressibility in the model, an inviscid fluid will yield Euler equations, by removing the terms describing vorticity potential equations can be derived; further these can be linearized to yield the linearized potential equations etc. Here will be presented only the general form of the unsteady Navier-Stokes equations in their conservation form, as also set up in ANSYS CFXII.O, the CFD package used as the modelling tool for all of the here presented numerical simulations ${ }^{\mathrm{II}}$.

The instantaneous equations of mass, momentum and energy conservation in a stationary frame can be written as follows $[\boldsymbol{B}],[\boldsymbol{g}],[\boldsymbol{I} \boldsymbol{O}]$ :

\section{The Continuity Equation (Conservation of Mass)}

A continuity equation describes the transport of a conserved quantity (mass, energy, momentum, etc). By making a mass balance of the flowing fluid over an infinitesimal volume element fixed in space, the formulation below is generated:

$$
\frac{\partial \rho}{\partial t}+\nabla \cdot(\rho \underline{U})=0
$$

$\rho$ here is the fluid density and $\underline{U}$ is the fluid velocity (three components in orthogonal Cartesian coordinate system: $u, v$ and $w$ ). $t$ represents the time coordinate.

\section{The Momentum Equation}

Newton's law of conservation of momentum states that the rate of change of momentum of a material volume equals the total force exerted on the volume:

$$
\frac{\partial(\rho \underline{U})}{\partial t}+\nabla \cdot(\rho \underline{U U})=-\nabla p+\nabla \cdot \underline{\underline{\tau}}+\underline{S_{M}},
$$

where the viscous stress tensor, $\underset{=}{\underline{\tau}}$, is for a Newtonian fluid related to the strain rate by:

\footnotetext{
II Historically, only the momentum equations for a viscous flow were identified as the Navier-Stokes equations $[\boldsymbol{I I}]$. However, in the modern CFD literature, the terminology has been expanded to include the entire system of flow equations for the solution of a viscous flow - continuity and energy as well as momentum. Therefore, when the CFD literature discusses a numerical solution to the Navier-Stokes equations, it is usually referring to a solution of the complete system of equations.
} 


$$
\stackrel{\tau}{=}=\mu\left(\nabla \underline{U}+(\nabla \underline{U})^{T}\right)-\left(\frac{2}{3} \mu-\kappa\right) \delta \nabla \cdot \underline{U} .{ }^{12}
$$

$p$ here is the medium pressure, $\underline{S_{M}}$ is the external momentum source, $\mu$ dynamic viscosity and $\delta$ is the Kronecker delta. $\kappa$ is the dilatational viscosity, and is identically zero for monatomic gasses at low density, which, the here investigated gas - helium is [IO ].

\section{The Energy Equation}

The energy equation stems from the first law of thermodynamics applied to a fluid element moving with the flow, stating that the rate of change of energy of the fluid element during this process must equal the net flux of heat into the element plus the rate of work done on the element due to body and surface forces $[\boldsymbol{I} \boldsymbol{O}]$. In accordance with this definition, the energy equation is formulated in $\mathrm{CFX}$ as ${ }^{\mathrm{I3}}$ :

$$
\frac{\partial \rho h}{\partial t}+\nabla \cdot(\rho \underline{U} h)=\nabla \cdot(\lambda \nabla T)+\frac{\partial p}{\partial t}+\underline{U} \cdot \nabla p+\underline{\tau}: \nabla \underline{U}+\underline{U} \cdot \underline{S_{M}}+S_{E} .
$$

Here $\lambda$ is the thermal conductivity, $T$ is the fluid temperature, $\underline{U} \cdot \underline{S_{M}}$ work due to the external momentum sources and $S_{E}$ is the external energy source. The term $\underline{\tau}: \nabla \underline{U}$ represents the irreversible viscous dissipation.

Finally, the static enthalpy $h$ is related to the internal energy $e$ by:

$$
h=e+\frac{p}{\rho}
$$

so Equation (3.4) can be further written as:

$$
\frac{\partial \rho e}{\partial t}+\nabla \cdot(\rho \underline{U} e)=\nabla \cdot(\lambda \nabla T)-p \nabla \cdot \underline{U}+\underline{\tau}: \nabla \underline{U}+\underline{U} \cdot \underline{S_{M}}+S_{E} .
$$

I2 This notation is in accordance with the CFX Theory Guide [9], since this chapter directly references to the ANSYS code. In Bird et al. [Io], the viscous stress tensor is defined with the opposite sign, thus: $\underline{\underline{\tau}}=-\mu\left(\nabla \underline{U}+(\nabla \underline{U})^{T}\right)+\left(\frac{2}{3} \mu-\kappa\right) \delta \nabla \cdot \underline{U}$, which further reflects the sign of the viscous dissipation term in the energy equation.

I3 In the CFX Theory Guide [9] also referred to as the thermal energy equation. 
The set of seven unknowns $(u, v, w, p, T, \rho, h)$ is presented in the above five equations. Reading from the thermodynamic laws and with the assumption of applicability of the ideal gas relation, for simple systems there are at most two independent state variables, on which the others depend algebraically. The set is thus closed by adding two algebraic thermodynamic equations; in ANSYS CFX, the flow solver calculates fluid pressure and static enthalpy.

The thermal equation of state correlates density as a function of pressure and temperature. For an ideal gas, this relationship is described by the Ideal Gas Equation:

$$
\rho=\frac{M\left(p+p_{r e f}\right)}{R_{0} T}
$$

where $M$ is the molecular mass of the gas, $p$ and $p_{\text {ref }}$ the relative and reference pressure in the system, and $R_{0}$ is the universal gas constant.

The second closure equation relates the enthalpy to the pressure and temperature:

$$
h-h_{0}=\int_{\mathrm{T}_{0}}^{\mathrm{T}} c_{p} d T,
$$

or, translated to the internal energy:

$$
e-e_{0}=\int_{\mathrm{T}_{0}}^{\mathrm{T}} c_{v} d T .
$$

The specific heat capacity at constant pressure $c_{p}$ can in general be a function of both pressure and temperature of a concerned medium, but for an ideal gas it simplifies to change as dependent only on temperature:

$$
c_{p}=c_{p}(p, T), c_{p, \text { ideal gas }}=c_{p}(T) .
$$

For a non-ideal gas, presented state relations become somewhat more extensive ${ }^{\mathrm{I}}$, but having in mind that helium, as a fluid used in this research, behaves as an ideal gas in a very wide bandwidth of thermodynamic conditions, other equations of state will not be elaborated on further here.

\footnotetext{
I4 In ANSYS CFXII.o, the Redlich Kwong equation of state is available as a built-in option for simulating real gases. It is also available through several pre-supplied CFX-TASCflow RGP files. The Vukalovich Virial equation of state is also available by using CFX-TASCflow RGP tables [9].
} 
For additional reading on the above topics, reader is referred to sources $[\boldsymbol{8}],[\boldsymbol{g}],[\boldsymbol{I} 2]$ and [I3].

\section{Conjugate Heat Transfer in the Solid}

ANSYS CFX is one of the advanced CFD packages that allows for incorporation of solid domains and modelling of the fluid-solid interaction within one simulation model. In a solid domain, the equation for the conservation of energy is simplified since there is no flow within, or in and out of a solid, and thus conduction is essentially the only mode of energy (heat) transfer. The heat equation in the solid is hence described with the following transport equation:

$$
\frac{\partial}{\partial t}\left(\rho c_{p} T\right)=\nabla \cdot(\lambda \nabla T)+S_{E}
$$

Fluid and solid domains are coupled in ANSYS CFX via conservative domain interfaces, allowing for momentum and energy transfer across the boundaries. Two (fluid-solid) and three (fluid-solid-cooling fluid) domain models are not reported in this thesis, although initial work in meshing and investigation of this functionality has been performed [I4]. Expansion to the coupled models would definitely be one of the suggested directions for continuation of the here presented work and further efforts investment.

\section{I.I Turbulence Models and DNS}

ANSYS CFX, like most of the modern CFD tools, allows for employment of turbulence models in simulating the flows. Turbulence is a very complex stochastic phenomenon and the fluid dynamics community is in constant search for new advances and findings in understanding and describing it, and creating cheaper and more accurate instruments for its prediction. Direct Numerical Simulation-DNS approach uses no turbulence modelling; it solves the Navier-Stokes simulations at the level of the smallest scales of interest ${ }^{15}$. In accordance with this principle, it is necessary to ensure sufficient spatial and temporal resolutions for such simulations, which naturally steeply increases any computational efforts and limits its utilisation. As alternatives, when the scope of the research allows it and/or the simulation costs are not available, solutions of the Navier-Stokes equations can be simplified in a manner that either describes the turbulence in the terms of time- or ensemble-mean flow properties and their fluctuating components (Reynolds Averaged Navier Stokes - RANS simulations) with the closure models introduced, or filters the smaller-contributing turbulent structures - vortices or eddies and implicitly accounts for them, while explicitly solving the large-scale turbulence (Large Eddy Simulations - LES).

\footnotetext{
I5 These considerations are presented in subchapter 5.2: Kolmogorov Scale.
} 
Many improvements and hybrids of these two main methodologies are applicable and suitable to (specific) CFD problems, and very broad literature is in this respect available. Reader is strongly referred to [I5], [II $],[\boldsymbol{I} \boldsymbol{O}],[\boldsymbol{I} 7]$.

In the previously published work $[\boldsymbol{I} \boldsymbol{\delta}]$, we explored the employment of the $k-\omega$, ScaleAdaptive Simulation - SAS [9] and LES models for solving similar problems; nevertheless this was later abandoned because of the apparent absence of the actual developed turbulence in the compressed fluid for the gas spring operation investigated in this research $^{\mathrm{I}}$, and the capability of the ANSYS CFX DNS approach to numerically solve the attended fluid flow problems. Mass and energy conservation aspects, and the numerical accuracy related to the used models is elaborately discussed in Chapter 4. All of the here presented results are thus obtained without the use of any approximating models, but with the physical variables solved at the nodes of every meshed cell in a modelled domain, using DNS.

\subsection{Numerical Discretization}

ANSYS CFX is a Finite Volume Method - FVM code. To obtain solutions for governing equations, a numerical approach must be adopted, whereby the equations are replaced by algebraic approximations and solved using a numerical method. This approach involves discretizing the spatial domain into finite control volumes using a certain mesh. The governing equations are integrated over each mesh control volume, so that the relevant quantity (mass, momentum, energy, etc.) is conserved in it in a discrete sense. Solution is further pursued marching through the time scale, i.e. performing the above mentioned for every iteration or timestep.

Firstly the computing domain needs to be meshed; i.e. disintegrated and translated into a bonded assembly of finite volumes, each of which has its unique form and address, and encloses its appropriating node. Figure 3.I shows a typical mesh sample with unit depth (i.e. presented two-dimensional here), on which one surface of the control volume is represented by the shaded area. Each node is surrounded by a set of surfaces that define the control volume, and an element with a discrete thickness will form a $3 \mathrm{D}$ surface. All the solution variables and fluid properties are stored at the element nodes.

Let us consider the general forms of the conservation equations for mass (Equation (3.I)), momentum (Equation (3.2)) and a passive scalar $\phi$, expressed in Cartesian coordinates [9]:

${ }^{16}$ Discussed in subchapters 5.2 and 5.4 . 


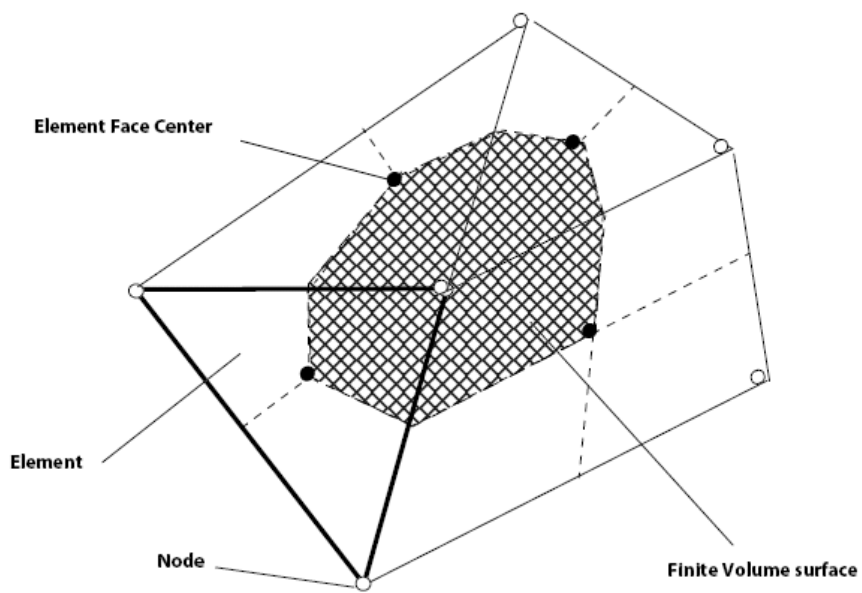

Figure 3.1: Control volume surface [9]

$$
\begin{gathered}
\frac{\partial \rho}{\partial t}+\frac{\partial}{\partial x_{j}}\left(\rho U_{j}\right)=0 \\
\frac{\partial}{\partial t}\left(\rho U_{i}\right)+\frac{\partial}{\partial x_{j}}\left(\rho U_{j} U_{i}\right)=-\frac{\partial p}{\partial x_{i}}+\frac{\partial}{\partial x_{j}}\left(\mu\left(\frac{\partial U_{i}}{\partial x_{j}}+\frac{\partial U_{j}}{\partial x_{i}}\right)-\frac{2}{3} \mu \frac{\partial U_{k}}{\partial x_{k}}\right)+S_{M} \\
\frac{\partial}{\partial t}(\rho \phi)+\frac{\partial}{\partial x_{j}}\left(\rho U_{j} \phi\right)=\frac{\partial}{\partial x_{j}}\left(\Gamma_{e f f}\left(\frac{\partial \phi}{\partial x_{j}}\right)\right)+S_{\phi}
\end{gathered}
$$

$\mu$ stands for the molecular viscosity, and $\Gamma_{e f f}$ for the effective diffusivity of a passive scalar $\phi$. These equations need to be integrated over a control volume, and Gauss' Divergence Theorem is applied to convert some of the volume integrals to surface integrals. If control volumes would not deform in time, the time derivatives could be moved outside of the volume integrals and the equations become:

$$
\frac{d}{d t} \int_{\mathrm{V}} \rho d V+\int_{S} \rho U_{j} d n_{j}=0,
$$




$$
\begin{aligned}
\frac{d}{d t} \int_{\mathrm{V}} \rho U_{i} d V+\int_{S} \rho U_{j} U_{i} d n_{j} \\
=-\int_{S} p d n_{j}+\int_{S}\left[\mu\left(\frac{\partial U_{i}}{\partial x_{j}}+\frac{\partial U_{j}}{\partial x_{i}}\right)-\frac{2}{3} \mu \frac{\partial U_{k}}{\partial x_{k}}\right] d n_{j} \\
+\int_{V} S_{M} d V \\
\frac{d}{d t} \int_{\mathrm{V}} \rho \phi d V+\int_{S} \rho U_{j} \phi d n_{j}=\int_{S} \Gamma_{e f f}\left(\frac{\partial \phi}{\partial x_{j}}\right) d n_{j}+\int_{V} S_{\phi} d V ;
\end{aligned}
$$

where $V$ and $s$ denote the volume and surface regions of integration, respectively, and $d n_{j}$ are the differential Cartesian components of the outward normal surface vector. The volume integrals represent the source or accumulation terms, and the surface integrals represent the summation of the fluxes.

The first step in the numerical solution of these integral conservation equations is to create a coupled system of linearized algebraic equations. This is done by converting each term into its discrete form. Consider, for example, an isolated mesh element like the one shown in Figure 3.2. For volumetric terms, this is done by approximating specific values in each sector and then integrating those values over all sectors that contribute to a control volume. Surface flow terms are converted into their discrete form by first approximating fluxes at integration points, ipn, which are located at the centre of each surface segment surrounding a $3 \mathrm{D}$ element of the control volume. Flows are then evaluated by integrating the fluxes over the surface segments that contribute to a control volume.

The discretized forms of the integral equations become:

$$
\begin{gathered}
V\left(\frac{\rho-\rho^{0}}{\Delta t}\right)+\sum_{i p}\left(\rho U_{j} \Delta n_{j}\right)_{i p}=0 \\
V\left(\frac{\rho U_{i}-\rho^{0} U_{i}^{0}}{\Delta t}\right)+\sum_{i p} \dot{m}_{i p}\left(U_{i}\right)_{i p} \\
=\sum_{i p}\left(p \Delta n_{i}\right)_{i p}+\sum_{i p}\left(\left[\mu\left(\frac{\partial U_{i}}{\partial x_{j}}+\frac{\partial U_{j}}{\partial x_{i}}\right)-\frac{2}{3} \mu \frac{\partial U_{k}}{\partial x_{k}}\right] \Delta n_{j}\right)_{i p} \\
+\frac{S_{M} V}{}
\end{gathered}
$$




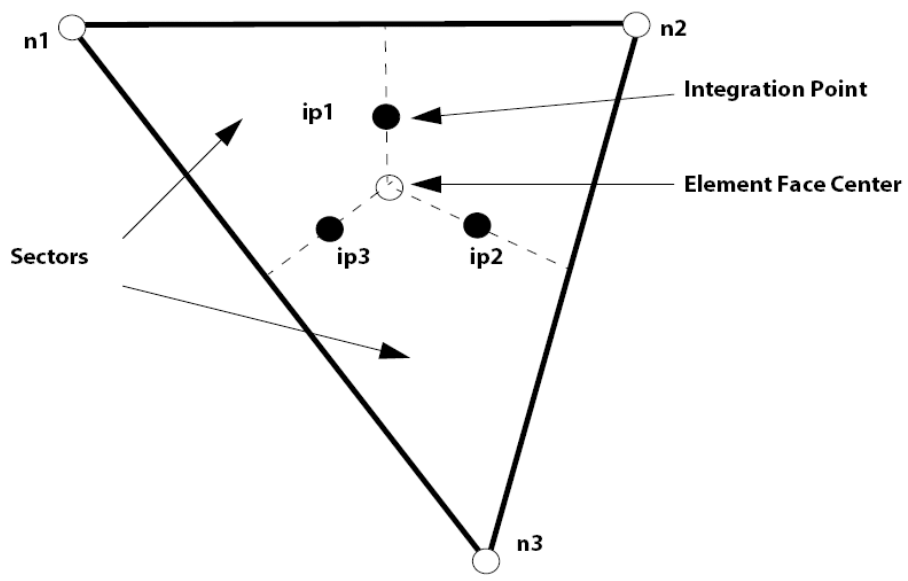

Figure 3.2: A mesh element [9]

$$
V\left(\frac{\rho \phi-\rho^{0} \phi^{0}}{\Delta t}\right)+\sum_{i p} \dot{m}_{i p} \phi_{i p}=\sum_{i p}\left(\Gamma_{e f f} \frac{\partial \phi}{\partial x_{j}} \Delta n_{j}\right)_{i p}+\overline{S_{\phi}} V
$$

where

$$
\dot{m}_{i p}=\left(\rho U_{j} \Delta n_{j}\right)_{i p}
$$

is the discrete mass flow through a surface of the control volume, $V$ is the volume of the specific control volume element, $\Delta t$ is the timestep, $\Delta n_{j}$ is the discrete outward surface vector, the subscript ip denotes evaluation at a discrete integration point, and the summations are over all the integration points of the control volume. Superscript ${ }^{\circ}$ refers to the old time level.

A Second Order Backward Euler scheme is used in all simulations presented in this thesis (also directly available in ANSYS CFXII.O). The transient term is then second-order accurate in time and, for control volumes that do not deform in time, the general discrete approximation of the transient term for the $\mathrm{n}^{\text {th }}$ timestep reads:

$$
\frac{\partial}{\partial t} \int_{\mathrm{V}} \rho \phi d V=V \frac{1}{\Delta t}\left(\frac{3}{2}(\rho \phi)-2(\rho \phi)^{0}+\frac{1}{2}(\rho \phi)^{00}\right) .
$$

Extension to the deforming mesh is discussed in the following section. 
For advection terms, High Resolution differencing scheme is employed in all simulations. The advection schemes implemented in ANSYS CFX are cast in the form:

$$
\phi_{i p}=\phi_{u p}+\beta \nabla \phi \cdot \vec{l}
$$

where $\phi_{u p}$ is the variable value at the upwind node, and is $\vec{i}$ the vector from the upwind node to the integration point ip. The High Resolution scheme sets $\nabla \phi$ equal to the control volume gradient at the upwind node and computes $\beta$ locally as close to 1 as possible without introducing local oscillations. The High Resolution scheme is both accurate and bounded since it is formally second-order accurate and only reduces to first order near discontinuities [9].

Spatial derivatives of all diffusion terms are evaluated using shape functions, also used to calculate geometric properties such as integration point coordinates and surface area vectors. Shape functions used in ANSYS CFX are linear in terms of parametric coordinates; overview for all supported mesh elements is given in $[\boldsymbol{B}]$ and [9]. For a fast reference here, a derivative of a variable $\phi$ in the $x$ direction at an integration point ip will be:

$$
\left.\frac{\partial \phi}{\partial x}\right|_{i p}=\left.\sum_{n} \frac{\partial N_{n}}{\partial x}\right|_{i p} \phi_{n}
$$

where $N_{n}$ is the shape function for node $n$ and $\phi_{n}$ is the value of the variable at node $n$. The summation is then done over all the nodes for a concerned element.

\subsection{Mesh Deformation}

This research concerns dynamic systems and moving boundaries and the created models thus utilize moving meshes. When the control volumes deform in time, the integral conservation equations presented above must be modified accordingly. In presented models the piston surface is logically a moving boundary, and consequently causes the displacement of all the domain's nodes and element faces.

Modifications in the governing equations obey the Leibniz Integral Rule [9], [I9]:

$$
\frac{d}{d t} \int_{\mathrm{V}(\mathrm{t})} \phi d V=\int_{\mathrm{V}} \frac{\partial \phi}{\partial t} d V+\int_{S} \phi W_{j} d n_{j}
$$

where $W_{j}$ is the velocity of the control volume boundary. The integral conservation equations (3.I5) - (3.I7) then become: 


$$
\begin{gathered}
\frac{d}{d t} \int_{\mathrm{V}(\mathrm{t})} \rho d V+\int_{S} \rho\left(U_{j}-W_{j}\right) d n_{j}=0 \\
\frac{d}{d t} \int_{\mathrm{V}(\mathrm{t})} \rho U_{i} d V+\int_{S} \rho\left(U_{j}-W_{j}\right) U_{i} d n_{j} \\
=-\int_{S} p d n_{j}+\int_{S}\left[\mu\left(\frac{\partial U_{i}}{\partial x_{j}}+\frac{\partial U_{j}}{\partial x_{i}}\right)-\frac{2}{3} \mu \frac{\partial U_{k}}{\partial x_{k}}\right] d n_{j} \\
+\int_{V} S_{M} d V, \\
\frac{d}{d t} \int_{\mathrm{V}(\mathrm{t})} \rho \phi d V+\int_{S} \rho\left(U_{j}-W_{j}\right) \phi d n_{j}=\int_{S} \Gamma_{e f f}\left(\frac{\partial \phi}{\partial x_{j}}\right) d n_{j}+\int_{V} S_{\phi} d V .
\end{gathered}
$$

The transient term accounts for the rate of change of fluid mass in the deforming control volume, and the advection term accounts for the net advective transport per unit time across the control volume's moving boundaries.

Spurious sources of errors are avoided by ensuring that the Geometric Conservation Law $(G C L)[20]$ is satisfied in the discretization of the transient and advection terms:

$$
\frac{d}{d t} \int_{V(t)} d V=\int_{s} W_{j} d n_{j}
$$

It states that the change in volume that is accounted for by the transient term must be consistent with the volume swept by the moving boundaries of the control volume. This is satisfied by using the same routines for both the control volume and swept volume calculations, rather than by approximating the swept volumes using the mesh velocities [9].

An example of a deforming meshed model grid, as computed in the simulations discussed here, is displayed in Figure 3.3, in three instances of a compression cycle: at BDC, halfstroke, and top-dead-centre (TDC). The accuracy of the here briefly described, and throughout the entire research employed numerical schemes, is evaluated in more detail on the actual models in Chapter 4. 
Chapter 3. CFD Theory
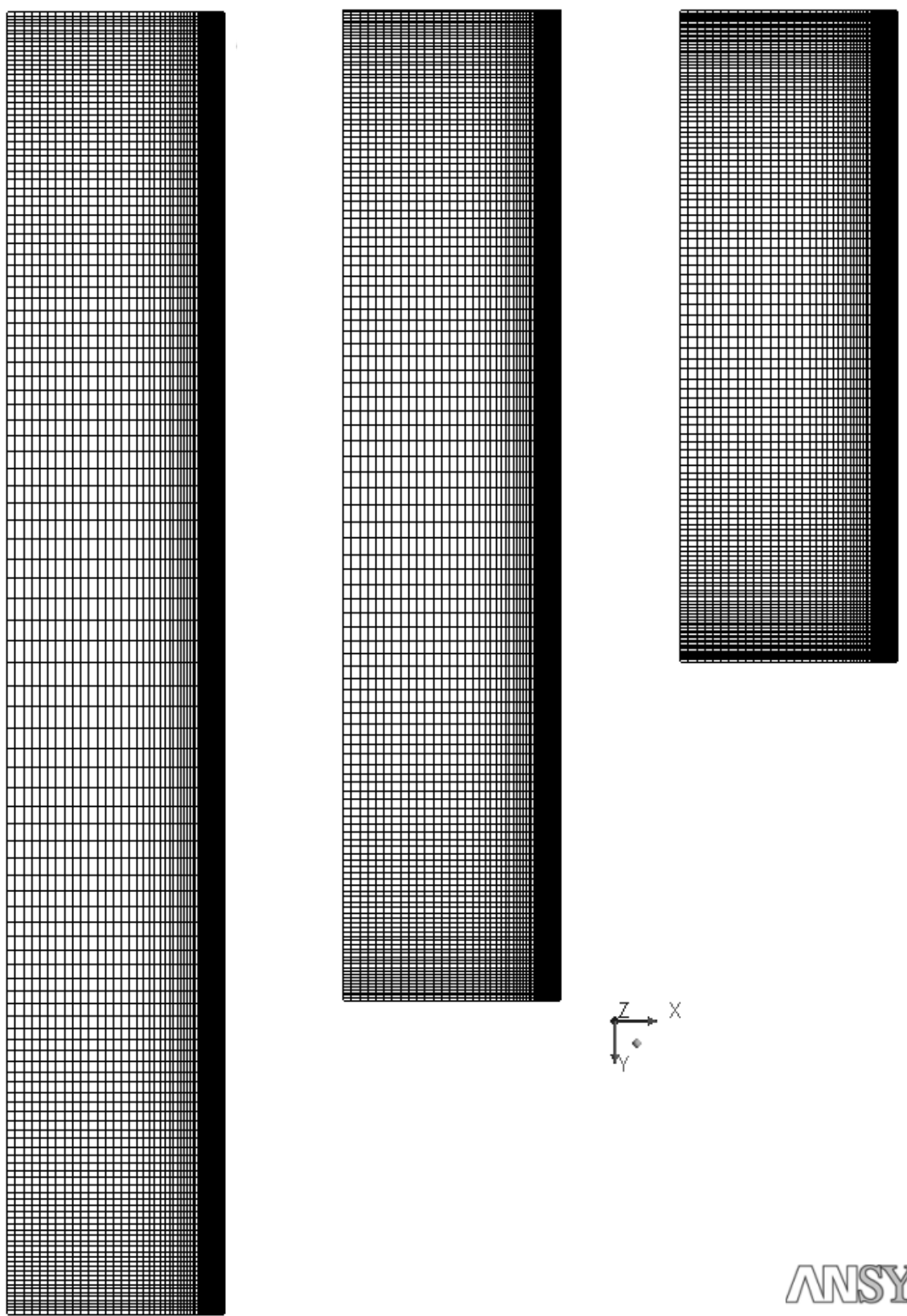

P

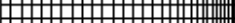

†

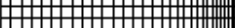

円

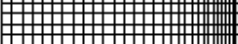

叶
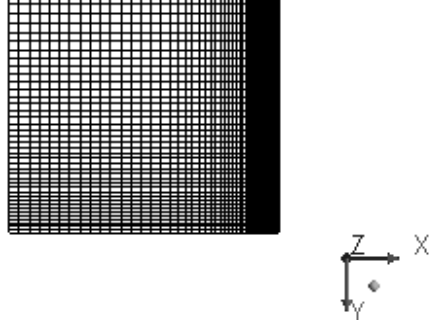

Figure 3.3: Snapshot of a computational domain deforming during one compression cycle, at three instances: BDC, half-stroke and TDC 


\subsection{Solution Strategy}

ANSYS CFX solver uses a coupled solver, which solves the discretized equations as a single system, using fully implicit discretization at any given timestep. The set of linear equations that arise from the above considered discretization of the domain can be written in the form $[\boldsymbol{B}]$ :

$$
\sum_{n b_{i}} a_{i}^{n b} \phi_{i}^{n b}=b_{i}
$$

where $\phi$ is the solution, $a$ the coefficients of the equation, $b$ the right hand side, $i$ is the identifying number of the node, and $n b$ means 'neighbour'. The node may have any number of such neighbours, so that the method is equally applicable to both structured and unstructured meshes. The solution of each set of field equations shown in the flow chart in Figure 3.4 then consists of two operations for each timestep: I - coefficient generation: the non-linear equations are linearized and assembled into the solution matrix that can be written in a general matrix form $[A][\phi]=[b]$, and 2 - equation solution where the linear equations are solved.

Reading Figure 3.4, the mesh displacement and hydrodynamic equations are solved in the iteration loops, controlled by the convergence criteria and the maximum number of iterations or coefficient loops for steady state and transient simulations, respectively. Only one inner iteration is performed per an outer iteration in steady state analyses, whereas multiple inner-iterations are performed per timestep in transient analyses. ANSYS CFX uses a multigrid accelerated Incomplete Lower Upper (ILU) factorization technique, and a particular implementation of Algebraic Multigrid called Additive Correction for solving the discrete system of equations. A multigrid process involves carrying out early iterations on a fine mesh and later iterations on progressively coarser virtual ones; the results are then transferred back from the coarsest mesh to the original fine mesh. Reasoning for this is the fact that the iterative solvers are only efficient at reducing errors which have a wavelength of the order of the mesh spacing. Using Algebraic Multigrid, the discretization is performed only once (for the original fine mesh), while using the virtually coarser grid larger errors are more efficiently eliminated, and long computing times or the necessity of meshing a geometry using a series of different mesh spacings is avoided.

For additional extensive information, please refer to [9], [2I], [22]. 


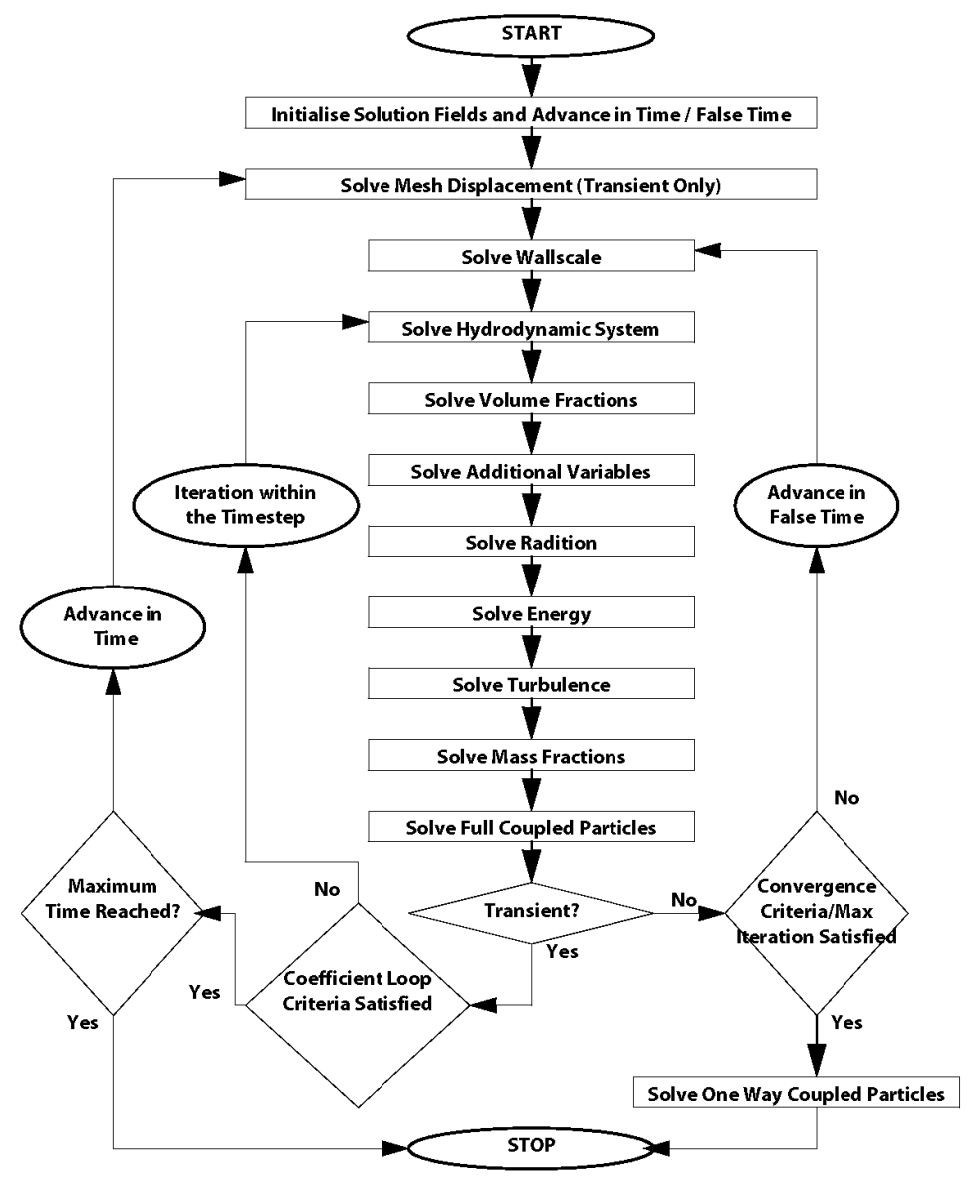

Figure 3.4: ANSYS CFX solving routine [9]

\subsection{Summary}

ANSYS CFX is a widely used CFD package, and a tool used during the course of numerical research reported in this thesis. In this chapter care has been taken to introduce the governing principles, general equations of interest that are included and solved in the presented models, and the discretization techniques and solving methods specific to the ANSYS CFXII.O code. Intention is to provide the reader with a stepping stone to understanding the simulations and results presented in the following chapters. For extended insight in CFD techniques or specifically ANSYS CFX computational code, the reader is recommended to the widely available literature, some of which are mentioned in the referred bibliography. 


\section{CHAPTER}

NUMERICAL MODELLING AND EXPERIMENTAL RESULTS

As described in the preceding chapter, CFD packages are created to enable 'numerical experiments', processes of examining the performance of a physical system in a virtual environment, without the pre-condition of physically building a scaled or a functional setup. Physical laws and processes are numerically described on a discrete computational grid, and with today's efficient and ample computer capacities allow for cheap and fast design processes and a wide base of available results. V alidation of these computational methods has to be performed in order to gain the insight in the extent of dependable modelling capabilities and complete the analyses, through comparison of numerical results to the available trustworthy benchmarks such as experimental data. In this thesis such a base is constructed on the base of experimental results, obtained from the experiments performed on the comparable physical setups at the Massachusetts Institute of Technology and the University of Twente.

In Chapter 3 the basics of CFD were explained; in Chapter 4, the applied numerical models created in ANSYS CFX11.0 are presented in details, starting from the geometry and meshing specifics, through initial and boundary conditions, to finally the results of the performed numerical simulations. These results are compared to the modelled range of experimental data and observations are discussed. This is also followed in Chapter 5 by a wider discussion on physical phenomena relying on the results of these simulations, and in Chapter 6 by comparing the numerical results to analytical and empirical correlations. 


\section{I Geometries and Spatial Discretization}

\section{I.I Modelled Geometries}

Construction specifics of two gas springs that are investigated in this research are elaborated on in details in Chapter 2. One gas spring is built and operated at the Massachusetts Institute of Technology (MIT) and the other, with two compression ratio constructions, is built at the University of Twente (UT). Both gas springs are operating with pistons undergoing semi-sinusoidal reciprocating motion in closed cylindrical volumes, sliding non-lubricated against the liner walls and compressing the enclosed operating gas. In accordance with the physical geometries, the fluid domain in the numerical models is always created as a perfect cylinder with the diameter equal to the physical model, and height corresponding to the machine with the piston in the bottomdead-centre (BDC) position (Figure 4.I). Solid structures surrounding the compressed fluid are presented in the numerical simulations by corresponding boundary conditions; models presented here refer only to the fluid mono-volumes. Computing domains are thus geometrically similar; with different diameter/stroke ratios for the two machines and compression ratios. An overview of the dimensions of interest for the simulations done for all geometries is presented in Table 4.I. Please note that $r$ in the table indicates the compression ratios.

Due to the simplicity of the experimental constructions and the axi-symmetry of the gas springs, and for the sake of reducing the necessary computational efforts, the numerical models could be further simplified. There is in reality no mechanism to act as the driving force for a flow in azimuthal direction in the gas springs, and, thus axi-symmetrical models can be assumed to give in first order a valid representation of a full geometry.

\begin{tabular}{cccccc}
\hline $\begin{array}{c}\text { Machine } \\
\text { dimensions } \\
{[\mathrm{mm}]}\end{array}$ & $\begin{array}{c}\text { Crankshaft } \\
\text { throw }\end{array}$ & $\begin{array}{c}\text { Connecting } \\
\text { rod length }\end{array}$ & Diameter & Stroke & $\begin{array}{c}\text { Dead } \\
\text { space } \\
\text { height }\end{array}$ \\
\hline \hline $\begin{array}{c}\text { MIT setup } \\
\begin{array}{c}\text { UT setup, } \\
\text { r=2 }\end{array}\end{array}$ & 38.10 & I83.00 & 50.80 & 76.20 & 76.20 \\
$\begin{array}{c}\text { UT setup, } \\
\text { r=8 }\end{array}$ & 26.00 & I 40.00 & 50.00 & 52.00 & 52.00 \\
\hline
\end{tabular}

Table 4.1: MIT and UT experimental setup dimensions 

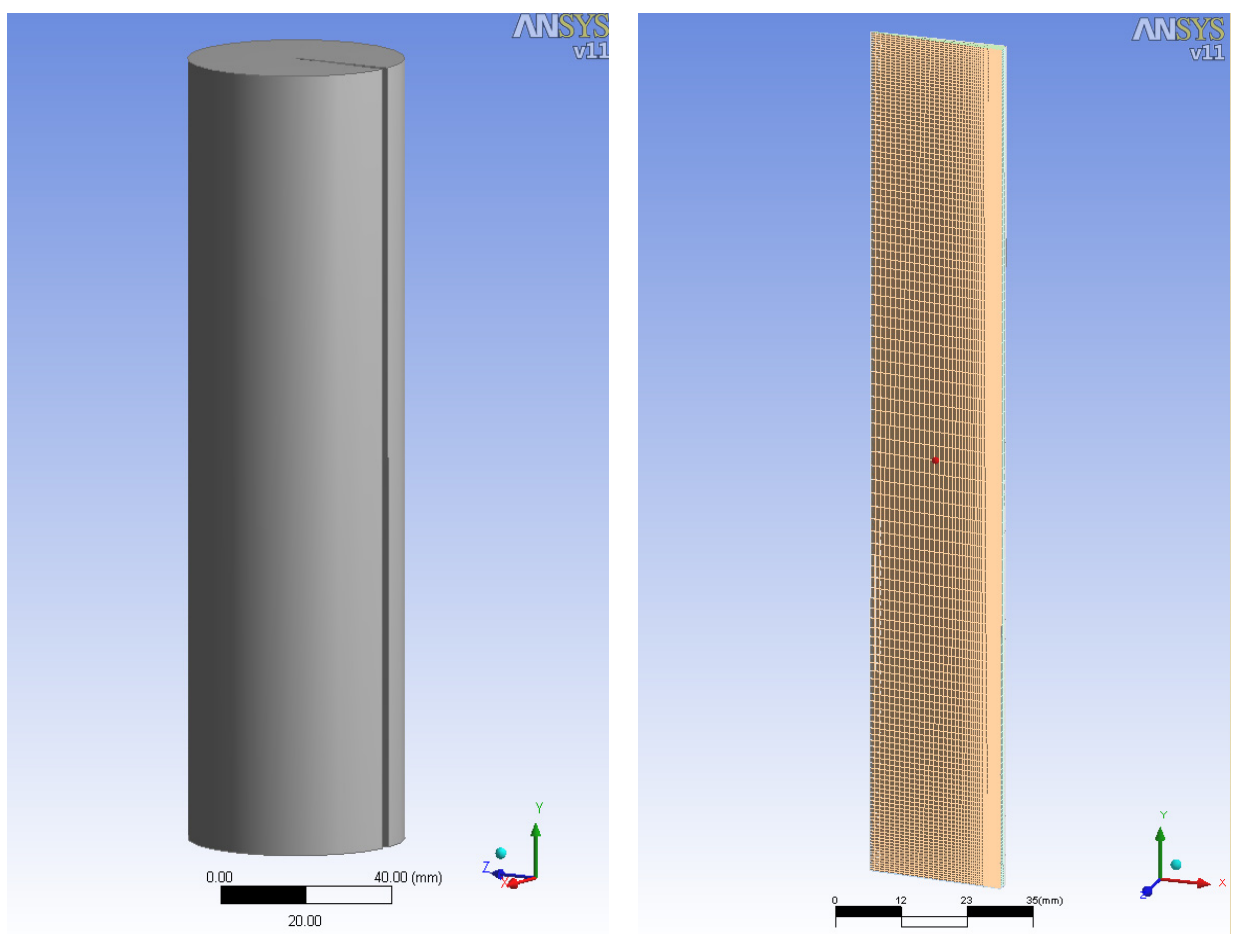

Figure 4.1: The compression-ratio 2 MIT gas spring model with a $5^{\circ}$ axial cut

Figure 4.2: Meshed 3D geometry of a gas spring model, $5^{\circ}$ slice used in the simulations
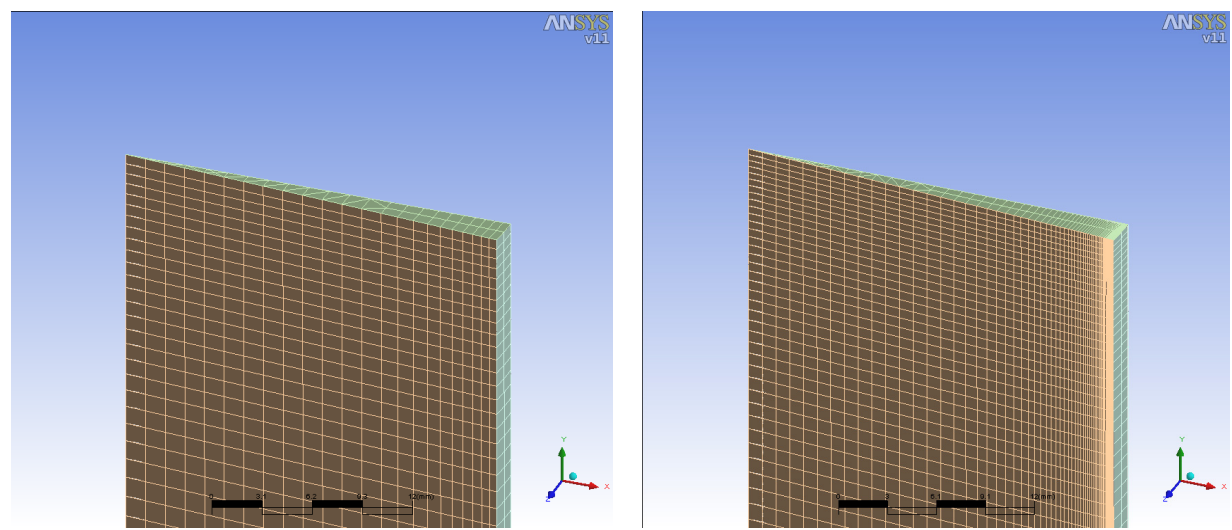

Figure 4.3: Top-corner view of the domain for 2 different mesh resolutions: MIT model with 6000 (left) and 28000 nodes (right figure) 
Creation of planar two-dimensional-mesh simulations is not supported in ANSYS CFXII.o for the fluid-flow modelling; instead, a small-thickness slab, or a small-angle three-dimensional domain slice can be created in ANSYS Design Modeller and meshed in CFX Mesh. Due to the axi-symmetry of the cylinder and feasibility of creating a very smallangle cut from the original model, a $5^{\circ}$ slice (Figure 4.2) is a selected domain for all simulations discussed here. This limit was achieved by gradually reducing the volume of the model from the full $360^{\circ}$ model downwards, and the $5^{\circ}$ slice showed to be a reasonable limit regarding the meshing requirements (discussed in the following section) and simulation costs, while there is no compromise on the thermodynamic results accuracy.

\section{I.2 Meshing}

For the spatial discretization of the models - meshing of the simulated domains, CFX Mesh application is used. Created meshes are structured in the axial and radial direction and generated using the Advancing Front option and Extruded 2D Mesh meshing strategy available in CFX Mesh, with the lower (piston) and upper (cylinder top) surfaces specified as Extruded Periodic Pairs. The mesh is strongly refined at the walls to capture the high temperature and density gradients expected at the wall boundaries ${ }^{17}$; Inflated Layers option in radial, and Symmetric Distribution in axial direction are used for this purpose, with the mesh spacing gradually growing towards the centre of the domain. The meshing specifics are optimized for every investigated geometry, and the effects of meshing resolution were examined on several control parameters. Gradual spatial refinement, from the initial coarse mesh ( 6000 nodes for the MIT model - Figure 4.3), was done to ensure the gridindependent solutions of the characteristic thermodynamic properties - gas pressure, gas temperature and wall heat flux, as well as to provide sufficient resolution both in the bulk volume and near the wall regions. The latter was necessary for the purposes of quantitative investigation of the radial temperature profiles and wall heat flux, but also for qualitative fluid-flow visualisation ${ }^{18}$. It should be noted that ANSYS CFX Solver proved to be very robust with respect to the spatial resolution, and gave convergence even for comparatively very coarse meshes.

For every geometry, the mesh was optimized on its critical simulation case - simulation for the highest operating frequency, being that, during the high speed operation, instabilities and effects of occurrence of turbulence are expected to have a negative effect on the numerical convergence and results. This was I6.67 Hz (IOOO RPM) for the MIT setup and $25 \mathrm{~Hz}$ for the UT setup. Resulting meshes are then used as generic, for the

\footnotetext{
${ }_{17}$ In accordance with the DNS approach, discussed in Chapter 3.

${ }^{18}$ Discussed in subchapter 5.4.
} 

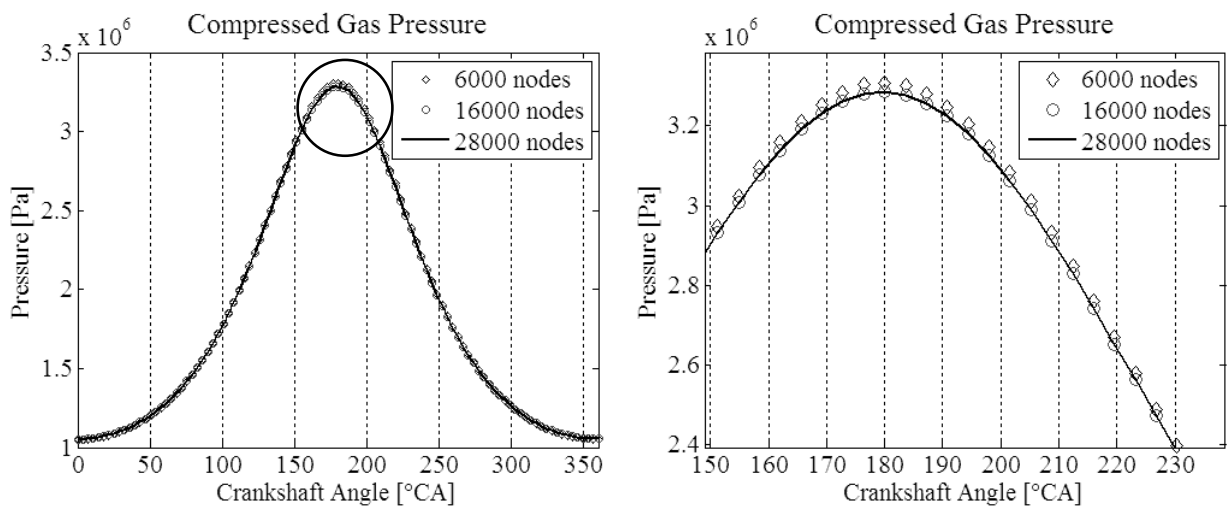

Figure 4.4: Effects of different mesh resolutions on the simulation results for the gas pressure
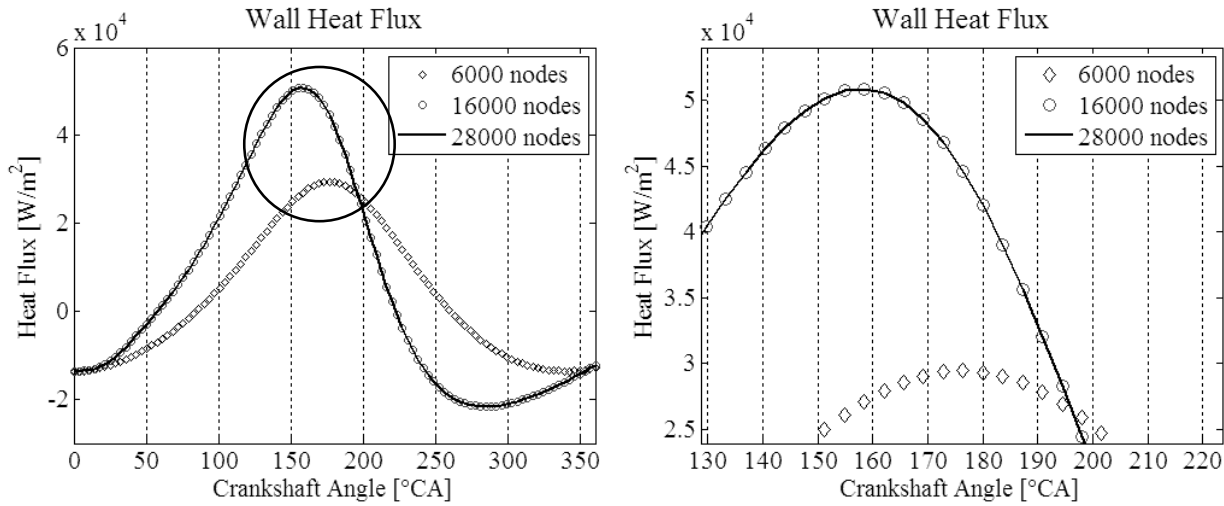

Figure 4.5: Effects of different mesh resolutions on the heat flux results

remaining range of investigated operating conditions applied to the same geometry (lower operating frequencies).

Figure 4.4 shows an example of the effects of the described mesh refinement procedure. Displayed are the pressure curves along one full compression-expansion cycle, for three resolutions of the MIT model, with $\sim 6000, \sim \mathrm{I} 6000$ and $\sim 28000$ nodes. Models show to be very insensitive with respect to the results for the simulated pressure: the error in the peak pressure results, for the displayed coarsest mesh, is no more than $\mathrm{I} \%$ of the cycle amplitude as compared to the finest, while the other two give identical results. However, as expected, the wall heat flux is much more affected by the meshing refinement (Figure 4.5), and most specifically by the resolution at the wall. The heat flux in the numerical simulations is derived directly from the temperature gradients in the gas at the wall 


\begin{tabular}{cccc}
\hline $\begin{array}{c}\text { Meshing } \\
\text { specifics }\end{array}$ & $\begin{array}{c}\text { MIT } \\
\text { model }\end{array}$ & $\begin{array}{c}\text { UT, r=2 } \\
\text { model }\end{array}$ & $\begin{array}{c}\text { UT, r=8 } \\
\text { model }\end{array}$ \\
\hline $\begin{array}{c}\text { \# of } \\
\text { nodes } \\
\begin{array}{c}\text { \# of } \\
\text { elements }\end{array}\end{array}$ & 27903 & I8I20 & I2I20 \\
\hline
\end{tabular}

Table 4.2: Meshing specifics for the MIT and UT models

boundaries, and in the case of the coarser meshes, cannot be correctly captured. The error for the coarsest mesh, close to the expansion end of the cycle is far over $100 \%$ and also out of phase; while for the other two, finer meshes, the heat flux results are again identical. After several consecutive revisions, the finite volume edge length in the centre of the volume for the MIT simulations and compression ratio 2, (Figure 4.3), was set to I $\mathrm{mm}$, with layers at the wall reducing down to $\mathrm{I} 2 \mu \mathrm{m}$ and the model so optimized to $\sim 28000$ nodes ( 20000 elements). A mesh with $\sim$ I6000 nodes already gave very accurate results for the heat flux as well as for the pressure ${ }^{19}$. Nevertheless, as mentioned, the refined mesh was further used in the simulations for the purpose of flow visualization, and correct capturing of the radial temperature profiles. For the UT simulations additional refinement had to be pursued at the wall boundaries, because of the occurring leakage of the gas at the piston-cylinder wall interface and a requirement for sufficient resolution of the leakage gap (will be discussed with the UT results - section 4.5.3). This reduced the first layer thickness at the cylinder wall down to I $\mu \mathrm{m}$. With this refinement and a cell thickness expansion factor slightly higher to compensate for the extremely fine mesh at the wall (I.3 in the boundary layer for UT model versus I.I for MIT model), the number of nodes for UT model was optimized to $\sim$ I 8000 (I 3500 elements) for compression ratio 2 , and $\sim \mathrm{I} 2000$ (9000 elements) for compression ratio 8 . This data is presented in Table 4.2. All elements are either prisms or hexahedral.

I9 It should be noted here that the mesh refinement was made with expansion factors in both directions - radial and axial - without taking specific care to keep the proportion between them constant. This could reflect to the fact that there is no gradual 'loss of accuracy' in Figure 4.5, going from the coarsest to the finest mesh. 


\subsection{Temporal Discretization}

The simulation models were also optimized for the sensitivity to temporal discretization settings. Choices for the temporal refinement were made in accordance with the recommendations given in the ANSYS Solver Guide [23], and consecutively increasing the number of timesteps per cycle by a reasonable factor. The temporal optimization was performed on the presented MIT model with the adopted 28000 nodes and again on the faster - I6.67 Hz (IOOO RPM) operating frequency simulation. Resolutions of IOO, 500, IOOO, 2000 and 4000 timesteps/cycle are examined and the results are shown in Figure 4.6
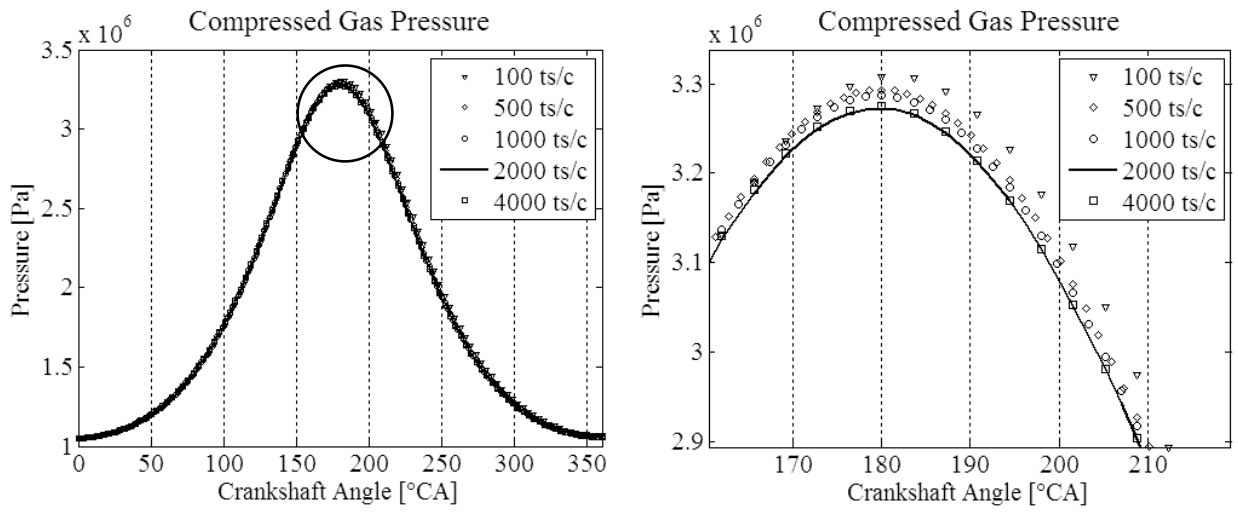

Figure 4.6: Effects of temporal refinement on the pressure results
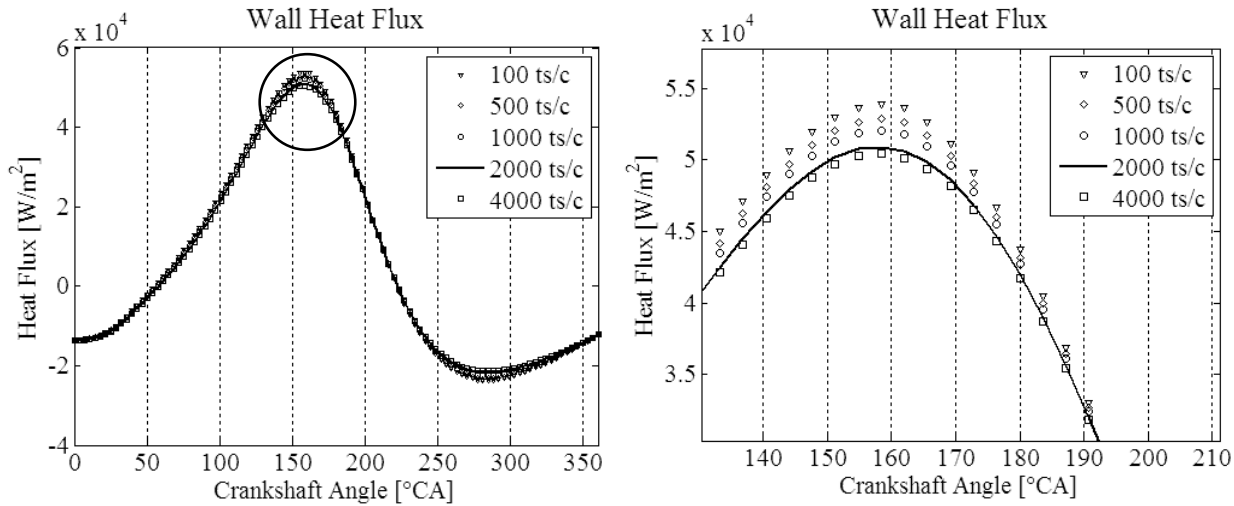

Figure 4.7: Effects of temporal refinement on the heat flux results 
and Figure $4.7^{20}$. As well as with the spatial refinement, a weak influence of the timestep size is observed on the computed values for the gas pressure. It amounts to a maximum of I. $46 \%$ error of the pressure amplitude at TDC, as compared between the two extreme temporal resolutions: IOO and 4000 timesteps/cycle. For the heat flux, though, a much smaller effect of this resolution is noticed than with the spatial refinement. The largest error is once more observed for IOO timesteps/cycle, and it amounts at its maximum $\left(\mathrm{I} 6 \mathrm{O}^{\circ}\right.$ CA) to approximately $6.6 \%$.

The obvious most accurate models, as visible in Figure 4.7, are the simulations with 2000 and 4000 timesteps/cycle. Smaller time-steps naturally cause an increase in the computational costs, and when evaluated on a typical simulation as observed here (described highest operating frequency run and 28000 nodes), a model with the temporal resolution of 4000 timesteps/cycle costs $\sim 22 \mathrm{hrs} /$ cycle CPU time on 3 processors, versus I 6 hrs/cycle for the model with 2000 timesteps/cycle. Error for the 'lighter' models with 2000 timesteps is $0.09 \%$ of the pressure cycle amplitude, and $0.8 \%$ of the heat flux peak. Adopting these as the acceptable errors in the view of significant computation costs saving, 2000 timesteps per cycle was chosen as a generic temporal resolution for all simulations presented in this thesis. The same two settings were re-examined for the UT model, and the results were very repeatable.

\subsection{Boundary Conditions and Initial Conditions}

As explained in previous paragraphs, the model shown in Figure 4.2 represents a sample of the compressed working fluid medium, i.e. the fluid domain. By defining the model's geometry and projecting a mesh to it, a model is physically described and prepared for a numerical experiment. Definition of a dynamic physical problem, to be set before the static model in such a numerical simulation, starts with the settings of the boundary and initial conditions.

20 ANSYS CFX uses a fully implicit solver, making no tight restrictions on the maximum Courant number (Courant-Friedrichs-Lewy - CFL condition) for convergence. Nevertheless, for the reader's feel, with the varying velocities and the deforming mesh, the cycle-maximum values of the Courant number for the selected 2000 timesteps/cycle are 0.I4 max RMS and 2.52 ensemble-max absolute value. Maximum absolute values are calculated at the very corner of the cylinder wall and cylinder top, where the spatial resolution is the smallest. The Courant number values for other temporal resolutions are inversely proportional to the number of timesteps, since the presented optimization is performed on a single grid. 


\subsection{Boundary Conditions}

Definition of the boundary conditions in a realistic numerical model has to rely on the properties of the physical situation, preferably known in advance and through the entire experiment. A full, $360^{\circ}$ numerical model (Figure 4.I) requires three boundaries to be physically described, i.e. for the

- cylinder wall - boundary in the azimuthal direction,

- $\quad$ ylinder top - upper boundary in the cross-sectional direction, and

- piston - lower boundary in the cross-sectional direction.

For the $5^{\circ}$ domain cut (Figure 4.2) that is presented in the preceding subchapters, two more boundaries need to be defined:

- $\quad$ two interface planes sharing the symmetry axis.

All solid boundaries (cylinder wall, cylinder head and the piston) in the simulation settings of all computational models are prescribed as wall boundaries, allowing no mass or momentum flux in the direction perpendicular to them. They are also assigned a no-slip condition, imposing the zero-velocity condition at all the walls. Interface planes are specified as Rotational Periodicity Interfaces, in this way ensuring the virtual multiplication of the $5^{\circ}$ geometry slice around the symmetry axis and allowing for the mass, momentum and energy periodic continuum of the model in the azimuthal direction.

The piston is the only moving body in the machine and, in accordance with this, the piston top, i.e. the lower domain boundary in the axial direction, is the only moving boundary in the computational model. All other boundaries, together with the coordinate frame, are set as stationary in space and time. Motion of the pistons for both setups (MIT and UT) is determined by the geometries of the driving machines guiding the pistons, i.e. the crankshaft throws and connecting rod lengths, copied from the real physical models (Table 4.I). With the rotating crankshaft, the displacement of the pistons can be derived from the geometrical relations [24]:

$$
P=a(1-\cos \theta)-l+\sqrt{l^{2}-a^{2} \sin ^{2} \theta}
$$

where $P$ is the piston displacement measured from its BDC position, $a$ is the crankshaft throw, $l$ is the connecting rod length and $\theta=\theta(t)$ is the crank angle also measured from the initial BDC state.

ANSYS CFXII.O allows for the moving/deforming mesh, i.e. the finite volumes are capable of compressing and redistributing in space during the simulation. In the models created and investigated in this research, every node position in every moment is specified through an explicit function. This is done in order to ensure uniform cell-distortion and 

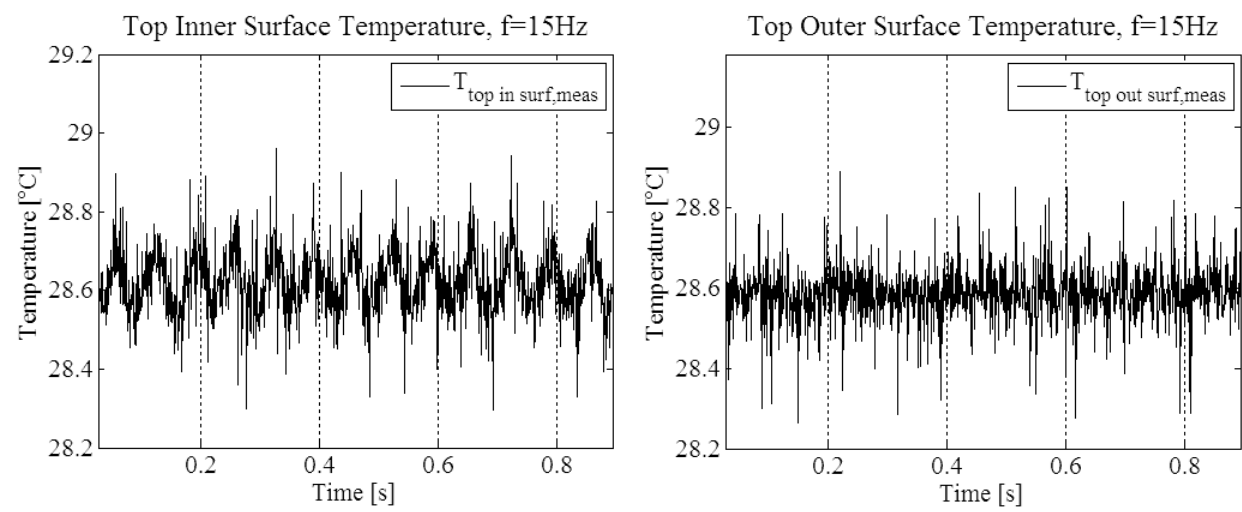

Figure 4.8: Measured surface temperatures on the compressed gas side (left) and the outer side (right) of the cylinder top

avoid the occurrence of 'negative' volume elements, due to numerical inaccuracies and a finite number of floating points in calculations of large deformations of small elements. This is done by correlating every node's and piston's initial position, to the piston's instantaneous coordinates in the observed moment of time, and thus displacing every node proportionally to the piston's displacement:

$$
N=\frac{y_{\text {Node, ini }}}{y_{\text {Pist }, \text { ini }}} \cdot P
$$

where $y_{P i s t, i n i}$ and $y_{N o d e, i n i}$ are the initial axial coordinates of the piston and the node in scope, and $N$ is that specific node's displacement in $y$ direction, measured from $y_{N o d e, i n i}$.

Next to the physical influence of boundaries on the position and motion of the fluid cells, their energy characteristics need to be correctly described as well. The piston surface in the numerical models for the MIT gas spring is set as an adiabatic wall, while other bounding surfaces were imposed constant temperatures of the coolant on the other side. This reflected the situation in the MIT test rig where the piston body was made of Micarta phenolic with thin brass surface layer and the cylinder wall and head were steel [2]. For the UT experiments, all wall boundaries are defined as walls with constant temperatures and allowing for the heat transfer. The values of these temperatures are specified on basis of the conducted experiments; they are measured with the thermocouples installed as described in Chapter 2 and then cycle-averaged. The approximation of the constant temperature of the walls is here adopted and considered to be allowed in accordance with the fact that the oscillations in the wall temperatures are very small compared to the temperature swings in the gas. In Figure 4.8 these are $\sim 0.2{ }^{\circ} \mathrm{C}$ on the inner surface of the cylinder top for a I5 Hz run. The bounding solids have a very high thermal inertia 
compared to the operating gas, and the small oscillation in the surface temperature will have negligible impact on the amplitude of the heat flux. This was also reported in earlier work, [25] and [26], in motored piston engines. In these experiments oscillation was shown to be no more than $\pm 0.5{ }^{\circ} \mathrm{C}[25]$ to $\pm 3{ }^{\circ} \mathrm{C}[26]$ in amplitude. Calculations of the effect of this assumption on the conditions in the examined MIT experiments were also performed by [27], and it was shown that the wall temperature can be safely regarded to as constant with a view to its effect on the wall heat flux.

\subsubsection{Initial Conditions}

Parameters of interest, and properties that thermodynamically determine a gas system, following the ideal gas equation: $p V=m R T$, are the pressure of the gas $p$, compressed gas volume $V$, mass $m$, (specific) gas constant $R$ and the gas temperature $T$. At least four of these properties have to be specified to thermodynamically describe any gas state, including the initial state for any numerical simulation. Being that the gas constant is a known gas property and the volume is uniquely dictated by the piston position, two extra parameters have to be determined; with mass being not an easily measured property, these have to be the pressure and the temperature of the gas. Gas pressure is a real-time obtainable parameter, and its instantaneous value (and thus also the initial value for the simulations, measurement at BDC), can be accurately sampled with available sensing equipment. The instantaneous temperature on the other side is very difficult to sample fast and accurately; subchapter 4.4 is devoted to this.

Measurements on both the MIT and UT experimental setups were logged once the gas spring had reached a cyclic steady state in its operation, i.e. when the cycle mean parameters of interest (gas pressure and temperature, wall temperature) remained constant - with variation less than $\mathrm{I} \%$. One full cycle was then isolated and taken as representative, and parameters from the beginning of the cycle, with the piston at BDC, were extracted. These parameters are the gas pressure, gas temperature, initial position of the piston and temperature of all the surrounding solids - piston, cylinder wall and cylinder top. These values were then taken as the input for the initial conditions in the respective numerical simulations.

At this point several remarks should be made, for full understanding of the limitations and approximations that were necessary to be done in order to correlate the experimental measurements to the results of the numerical simulations.

Firstly, all experimentally measured values hold coordinate information, i.e. they are sampled at discrete positions within a geometry. Contrary to this, initial values of pressure and temperature in the numerical simulations are volume parameters, i.e. a value applied to the entire gas domain assuming it to be uniform. Care has to be taken then to understand 
to what extent a parameter sampled at a single point can be translated to an isotropic initial value for a simulation. A pressure wave propagates with the speed of sound in a fluid, and the measured pressure was considered to be safely interpreted as homogeneous in all the compressed volumes, at every instance of a cycle and thus also for the initial value for the simulations ${ }^{21}$. The gas temperature, on the other hand, is highly position-dependent as will be also discussed in the following subchapter, and this kind of assumption cannot be valid. Effects of the adoption of uniform temperatures of the surrounding solid surfaces as the initial boundary conditions are minor and already discussed in the previous sections.

Further, when the numerical simulations are started, all gas velocity components are set to zero in the BDC starting position, since a correct mapping of initial velocity vectors in a compressed volume is impossible to impose. This is a close approximation, but of course not in exact accordance with the steady-state operation. To tackle this problem sufficient operating time (IO cycles) was simulated in order to also numerically reach the steady state, controlling the same parameters as in the physical experiment. Only then simulation results were sampled and taken as representative.

Finally, it should be emphasized that all initial values for the numerical simulations are very different to the measurements when the experimental machine is in the stand-still and these should in no way be combined. E.g. initial values of helium pressures for a numerical simulation are not identical to the measured charge pressures prior to the start of operation in the experiment. For the simulations, the initial pressure values are taken as the BDC measurements in the gas spring after a sufficient time of operation and steady state stabilization. Only the mean cycle pressure will be close to the charge pressure initially measured when the whole machine is at rest. Gas is, with this machine construction, unavoidably allowed to leak past the compressing piston, and although its displaced amount during one cycle is small and alternating in direction following the pressure potential, the overall leakage along the cycles before reaching a steady state is such that the mean pressures in the compressed and buffer space (above and below the piston) tend to equalize. This mass transfer is discussed and estimated in section 4.5.3. As examples of this problem: I.35 bar of charge (stand-still) pressure led to $\sim_{\text {I }}$ bar of BDC steady state pressure value during the experiment (initial for the simulations), or I.8 of

2I Speed of sound in helium (ideal gas) at room temperature is $c_{\text {helium }}=\sqrt{\frac{\gamma R T}{M}} \cong 1007 \frac{\mathrm{m}}{\mathrm{s}}$. For the gas spring operating at $25 \mathrm{~Hz}$ operating frequency, the wavelength of the acoustic waves will be $\lambda=\frac{c}{f} \cong$ $40 \mathrm{~m}$. Comparing it to the piston stroke: $\frac{\lambda}{s}=\frac{40}{0.052} \gg 1$, the statement that the pressure will be uniform in the domain during the oscillation of the piston is argumented and valid. This was also investigated by post-processing the numerical simulation for the fastest operation of the UT $r=2$ model, and the cycle-maximum spatial deviation over a sample plane showed to be less than $0.04 \%$ of the volume averaged cycle pressure amplitude. 
BDC pressure for a 3 bar charge. For the sake of clear comparison, term initial values will be further referred to as the set of initial parameters for the numerical simulations.

\subsubsection{Closure}

Considerations for the boundary and initial conditions set-up in the described models are presented. With all limitations and necessary approximations described above, in this way specified numerical models represent the physical setups to the highest extent possible, and are a base for performing the simulations and obtaining trustworthy results shown and analyzed in the following paragraphs.

A measurement results database for the MIT experiments was obtained by courtesy of Professor Alan Abram Kornhauser [2], and all boundary and initial conditions in the simulations of the MIT setup are thus based on these data. Recorded values in the MIT setup were the temperature of the captured gas measured directly prior to the start of the experiments and the pressure measured continuously during a compression cycle. The walls surrounding the compressed gas were conditioned and their temperature kept constant.

Experimental database for the simulations of runs performed at the University of Twente is obtained in continuous measurements during the experiments, and comprises a broader set of parameters. Gas pressure and temperature, as well as temperatures of the cylinder wall, cylinder top and piston were continuously monitored and recorded, and initial and boundary conditions for the simulations set accordingly, as explained.

All initial settings and boundary conditions, for all the simulations for MIT and UT experiments, are summarized in the tables presented at beginning of every respective section below.

\subsection{Estimating Initial Gas Temperature}

The compressed gas temperature in a compressing machine is, as well as the gas pressure, a highly dynamically changing value. With today's sensing equipment, capturing gas pressure at high sampling rates is well established and for standard and moderately advanced purposes an easily pursuable task. The situation is largely different with the temperature sensing equipment. Various types of thermal sensors for measuring gas temperatures do exist - thermocouples, resistors, thermistors, hot wire anemometry etc, but the performance of all of them is extremely dependent of the conditions of use, i.e. all of them are performing by the principle of heat exchange with the surrounding medium 
by mechanism of convection, and their accuracy and time responsiveness (time constants) are directly dependent on it. In an environment such as a helium gas spring, where the piston and the gas it compresses are undertaking a semi-sinusoidal motion and at its turnpoints are coming to rest, gas velocities approach zero and there occurs virtually no heat transfer between the gas and the sensing end of the equipment (other than the negligible processes of free convection, conduction in the sensor and gas apart, and radiation from a very thin and pure gas such as helium). The time constant of the sensor is directly dependent on the heat transfer coefficient between the measured medium and the sensor exposed surface:

$$
\tau=\frac{\rho_{s} V_{s} c_{s}}{h A_{s}}
$$

with $\tau$ the time constant, $V_{s}$ and $A_{s}$ the sensor's hot junction volume and area, $c_{s}$ and $\rho_{s}$ material's specific heat capacity and density, and $h$ the heat transfer coefficient ${ }^{22}$. Having this in mind, the small gas velocity (and thus $h$ ) will highly increase the reaction times of the sensing ends of the common equipment (thermocouples, resistors). For others, with possibly significantly lower thermal mass, but directly dependent on the medium velocity (hot wire anemometry), it will require an extremely extensive calibration procedure, and additional equipment for the measurement of velocities in the immediate vicinity of the sensor. Thus a direct, full-cycle measurement of the compressed gas temperature is very difficult, if not impossible, to implement in the here investigated types of processes. On the other hand, the temperature is, at every instant of a compression cycle, a highly position-dependent value, as it varies significantly along the radius of the compressed volume (considerations on this are available in Chapters 5 and 6), and this presents an added obstacle for gaining a dependable, one-value, directly measured representation of the gas temperature in the compression-expansion piston machines.

This being stated, certain approximations have to be introduced here, first of all a representative volume-averaged temperature has to be adopted as the initial value for the simulation since the thermal map cannot be known a-priori. For the MIT setup that utilizes a piston with sealing piston rings, the mass of the captured fluid can be assumed to be constant through the machine operation. In MIT experiments this enables the gas temperature to be correlated to the initial captured mass (volume at the known initial position of the piston and gas density for the initial temperature), and instantaneous values of the measured gas pressure and volume of the compressed gas (for the piston position at the observed moment) through the ideal gas law. The temperature obtained this way represents a 'thermodynamic' temperature, i.e. a value uniform for the entire compressed 
volume, and recalculated from the uniform gas pressure. This routine is used in the gas temperature determination for the MIT model simulations.

The construction of the UT setup is different in that the piston contains no sealing rings, and the fluid is unavoidably allowed to leak from the compressed space past the piston. In the UT experiments the temperature is sampled with miniature thermocouples as described in Chapter 2, extremely low in thermal mass ${ }^{23}$, but still not fast enough to accurately capture the highly transient changes in gas temperature. Before the beginning of an experiment, while the machine at rest, the temperature of the gas in the enclosed cylindrical volume is uniform and equal to that of its surrounding walls and can be accurately measured with the installed equipment, next to the measured pressure and known volume of the captured gas. Being that, for the simulations, the steady-state cycle parameters need to be known, the above information are not sufficient and cannot be adopted as the initial values for any operation cycle at a later point during the experiment. The enclosed mass of the compressed gas along the operation will be significantly different since mass exchange to- and from the compressed gas is unavoidable as described, and this distorts the direct link between the mass determined prior to the operation, the unknown transient gas temperature, and the measureable pressure and gas volume at later points. Thus a representative value for the gas temperature at the beginning of a cycle is in this case not straightforward to determine from the experiments. For this reason, an indirect method for determining the initial value for the gas temperature was developed and is described below.

The sensors used for the instantaneous measurement of the gas temperature are two miniature thermocouples ${ }^{24}$; one placed close to the cylinder wall $-6 \mathrm{~mm}$ in the gas, and a second placed radially in the centre of the compressed volume. Both thermocouples are axially positioned half-way of the 'dead' compressed volume, i.e. in the axial middle of the compressed volume with the piston in TDC position. Typical measurements are presented in Figure 4.9 for each thermocouple. It can be observed that the effect of the finite thermal mass of the hot junctions of the thermocouples is obvious; the amplitude of the measured temperature dampens out with the increase of operating frequency, which is directly opposite to a realistic situation - with the increase of operating speed both pressure and temperature amplitudes will actually rise. Also the maximum measured signal is observed to move towards a larger crank angle with the increasing frequency. The phenomenon that occurs here is that the sensor gets exposed for the longer period of time

22 Time constant of the miniature thermocouples installed in the UT experimental setup, for the maximum piston velocity occurring in examined experiments and developed flow is in the order of I.8 ms.

23 For the Chromel-Constantan E-type miniature thermocouples installed in the UT experimental setup with the patented MicroDisc ${ }^{\mathrm{TM}}$ junction, thermal mass amounts to $\sim \mathrm{Ie}^{-6} \mathrm{~J} / \mathrm{K}$.

24 Details in section 2.2.2 and Appendix C. 

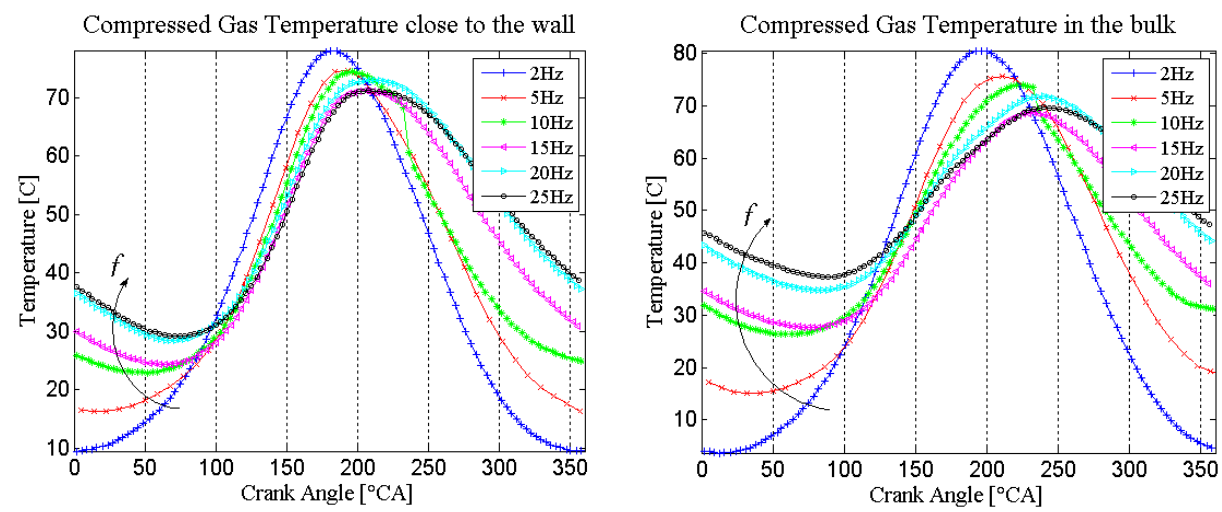

Figure 4.9: Measured gas temperatures for different operating frequencies and same initial conditions

to the slower changing impacts for the slower runs, and the measurement becomes more accurate. With the higher operating frequencies, the negative effect of the sensor's finite thermal constant in relative comparison to the time scale of the changes becomes larger, and the measured signal fails to follow its potential - the gas temperature.

Nevertheless, although the temperature signal was obviously not following the exact temperature-time curve, a concept is developed that, at the measured extremes, i.e. at the moments of change of sign of the recorded signal gradient, the sensor (no matter how slow it would be), has to record the exact actual temperature of the gas. At that point the potential for cooling down (or warming up) of the thermocouple junction is exhausted the actual gas temperature has 'passed' the temperature of the hot junction, and the thermocouple starts warming up (or cooling down). The routine for recalculating the gas temperature at the start of a steady-state compression cycle is then based on determination of the mass of the compressed gas in the moment of change of the recorded signal gradient, in the vicinity of an observed cycle (Figure 4.IO). The temperature and pressure signal are recorded synchronously (as for all measurements), and then for a temperature zero-gradient moment in the bulk-gas temperature measurement (Figure 4.IO top left), the time and the temperature value are isolated. For that time, the gas pressure is read (not necessarily at the BDC), and from these two values and the instant volume obtained from the piston position and defined by the crank angle, the captured mass is recalculated through the ideal gas law. For a steady-state BDC pressure in immediate vicinity of the observed cycle, the BDC initial temperature is then calculated with this obtained helium mass. In this way, the system of the initial conditions for a numerical simulation starting at $\mathrm{BDC}$ piston position is defined.

The accuracy of the presented method is initially dependent on the accuracy of the pressure reading and the time-synchronisation of the recorded signals. These are already 

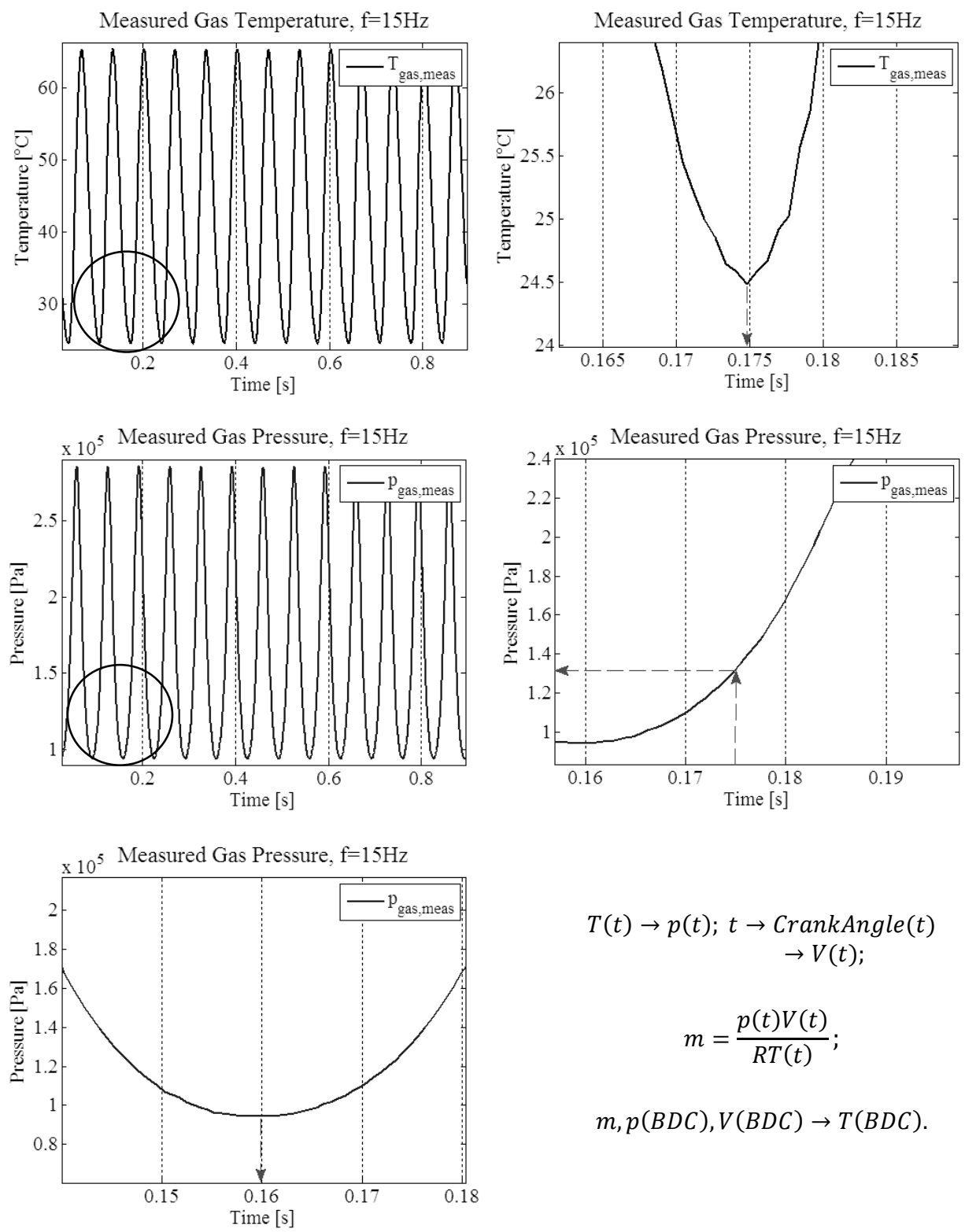

$$
\begin{gathered}
T(t) \rightarrow p(t) ; t \rightarrow \underset{\rightarrow V(t) ;}{\text { CrankAngle }(t)} \\
m=\frac{p(t) V(t)}{R T(t)} ; \\
m, p(B D C), V(B D C) \rightarrow T(B D C) .
\end{gathered}
$$

Figure 4.10: Procedure for estimating the initial gas temperature 
Measured and Simulated Temperature, $\mathrm{f}=2 \mathrm{~Hz}$

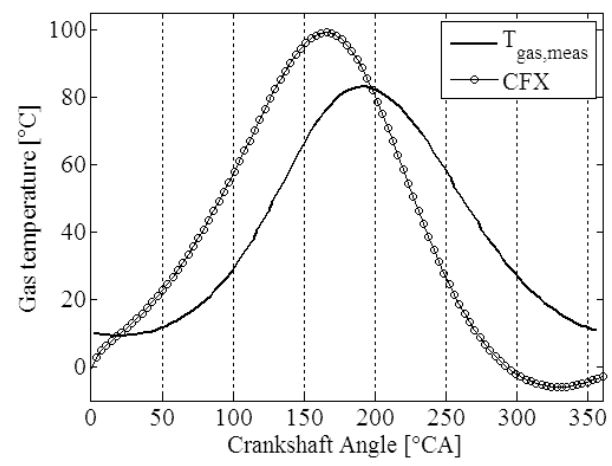

Measured and Simulated Temperature, $\mathrm{f}=25 \mathrm{~Hz}$

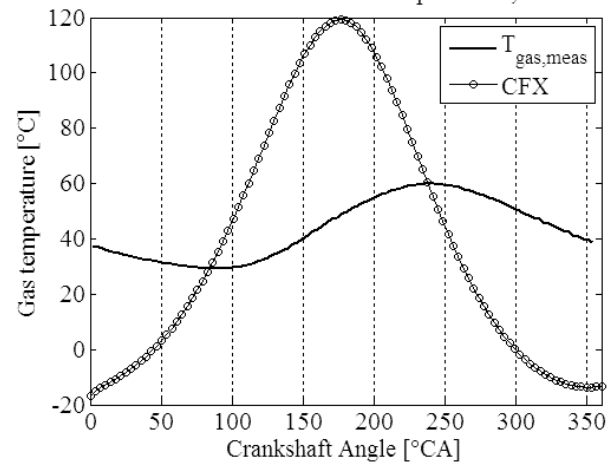

Figure 4.11: Validation of the initial temperature estimation method: comparison of the measured signal and the simulated gas temperatures for $2 \mathrm{~Hz}$ and $25 \mathrm{~Hz}$ runs; the signals cross at the peaks of the measured signal

discussed in Chapter 2, and for the scope of these evaluations can be regarded as negligible.

Another approximation that needs be mentioned is imposed by adopting the sampled bulk-gas temperature, at only one point in the compressed volume as a volume-mean temperature for the entire fluid domain. The error due to this approximation is difficult to quantify, and is related to the radial temperature profiles in the gas, dependent on the operating frequency. Temperature of the gas in the vicinity of the bounding solid walls is not directly related to the gas core temperature, with the layers in between again behaving very differently for different compression speeds. Much more on this is presented in Chapters 5 and 6.

Nevertheless, an intuitive method for validation of the method for evaluating the initial gas temperature presented here was the comparison of experimentally measured temperatures (signals from the sensor) to the end results of this research - the simulated gas temperatures at the coordinates of the sampling points of measurements. These results are typically presented in Figure 4.II for the two ends of the operating range of the UT experiments $-2 \mathrm{~Hz}$ and $25 \mathrm{~Hz}$. Control parameters here are the crossing points of the two curves; in accordance to the presented evaluation principle, as long as the signal and 'real' curves cross at the peaks of the recorded measured signal, the above stated concept confirms its validity. As shown in Figure 4.I I, this is to high extent true. 


\subsection{Results}

\subsection{Computational Procedure}

Since the full-form Navier-Stokes equations are numerically solved in ANSYS CFX, as explained in Chapter 3 and in [23], a very wide spectrum of physical properties is directly readable and producible from the results of the numerical simulations. Of interest here are parameters like the mass conservation and medium density, volume of the compressed finite volumes at every instant of the compression cycle, instantaneous values of gas pressure and gas temperature of every node, to finally the gas velocity vectors and the wall heat flux. This allows for very broad thermodynamical and fluid-mechanical considerations, some of which are presented in following paragraphs and chapters. For the purpose of validation of the numerical models and their comparison to the experimental data, gas properties that are taken as typical for the immediate interest of this research are the instantaneous gas pressure and the instantaneous heat transfer to the surrounding solid walls. In the following paragraphs these two properties are examined and plotted against the experimentally obtained values, and results are discussed.

\section{Absolute Pressure}

Experimental results from the gas pressure measurements are obtained from the installed equipment described in Chapter 2, using the advanced pressure sensors and then manipulating the recorded signals to include the sensor calibrations. These values are then presented as measured absolute pressure of the compressed gas.

In the numerically obtained results the pressure is calculated for every finite volume of the modelled domain; nevertheless, as previously explained in section 4.3.2, the gas pressure can be considered to be volume-uniform in the time-scales of concern here, and thus the gas pressure is averaged over the domain cells, and single volume-averaged values of calculated absolute pressures at every instant of a compression cycle are here referred to as "CFX" results. These are then plotted and compared to experimentally obtained pressures, and a discussion is given.

\section{Wall Heat Flux}

The second property that was investigated here is the instantaneous, area-averaged wall heat flux. For the experiments performed at MIT, the only available set of experimental data was the set of instantaneous pressure measurements (function of time), elapsed from the start of operation, and the experiment initial values of the gas temperature and of the 
surrounding walls. For the UT setup, a method for accurately estimating the heat flux from the obtained measured data was not fully developed during the course of this research ${ }^{25}$. Wall heat transfer thus had to be indirectly evaluated by calculating the instantaneous piston work and gas internal energy, and by the estimating the energy balances according to the First Law of Thermodynamics. This method was also suggested in [2] and is in details explained in this thesis in Appendix B, and is a straightforward means of additional validation of performance of numerical models. It has to be noted though, that the wall heat flux derived in this way is not a local property, as it is derived from the internal energy and it can only be understood as a domain area-averaged value. Nonetheless, since this heat transfer rate was the only considered in this thesis, experimental results manipulated with the above explained method will here be termed "the measured" data.

The numerically simulated wall heat flux on the other hand is a parameter obtained directly by the CFXII.o code, and it is calculated for every element face at the virtual fluid-wall interface, from the temperature gradient of the fluid at the wall. As such, calculated wall heat flux is not per se uniform (which is also the case in reality), but more intensive in the regions of higher velocities and less in the 'dead' zones ${ }^{26}$ (Figure 4.I2). Still, for the comparison to the measured heat flux (terminology explained above), numerical results for the wall heat flux are also area-averaged over the computational fluid domain's outer surface, and presented as a unique value for every time-step. These values are then named "CFX" wall heat flux, and compared to the measured heat flux.

\section{Numerical Conservation}

After the numerically defined geometries were meshed and the models set-up as described in subchapters 4 .I to 4.3 , and prior to finally performing the full simulations, the performance of these numerical models was initially tested. As a general criterion for correct set-up of the presented simulations, the mass and energy conservation during the compression process are verified on models with all walls set as adiabatic boundaries. In such a model, the energy has to be conserved in every moment of the compressionexpansion cycle. This then can be validated by the general adiabatic thermodynamic

\footnotetext{
25 Development of a method for correlating the sampled cylinder top surface and in-wall temperatures to the instantaneous heat transfer rates is in the suggested scope of the continuation of this project.

${ }^{26}$ In this way, numerical solvers can be utilized for various important thermal analyses. 'Thermally active' areas in a geometry can be identified, and optimisation of cooling (heating) of the solid can be preformed accordingly. This can also help in the identification of the hot-spots in a geometry and prediction of the correlated thermal distortions. Finally, the directly measured heat flux with a measuring element such as installed in the UT setup is in this way numerically simulated, and the results can be compared at specific coordinates (at the measuring points).
} 


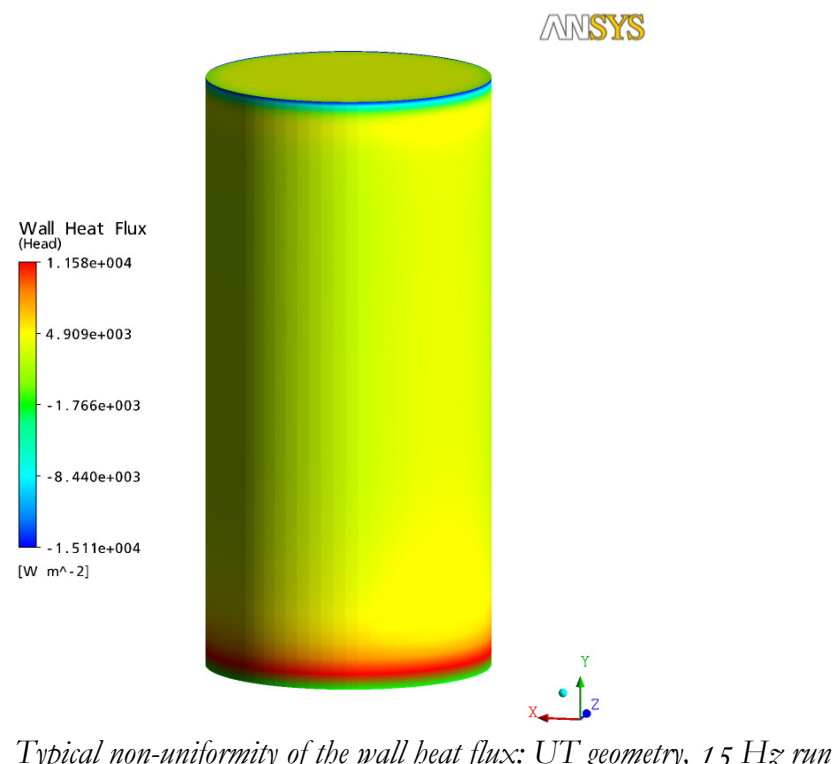

Figure 4.12: Typical non-uniformity of the wall heat flux: UT geometry, $15 \mathrm{~Hz}$ run

analogy $\left(p V^{\mu}=\right.$ const, $x$ being the adiabatic exponent), controlling the compressed gas pressure over a period of IO operating cycles. The analytic analogy is derived starting from the initial pressure and volume as set-up in the simulations, and then by directly calculating the pressure at each new timestep, for the adiabatic compression exponent and known volume change. Results of the mass and energy conserving numerical simulations have to be identical to this. Examples of such a procedure are presented in Figure 4.I3 and

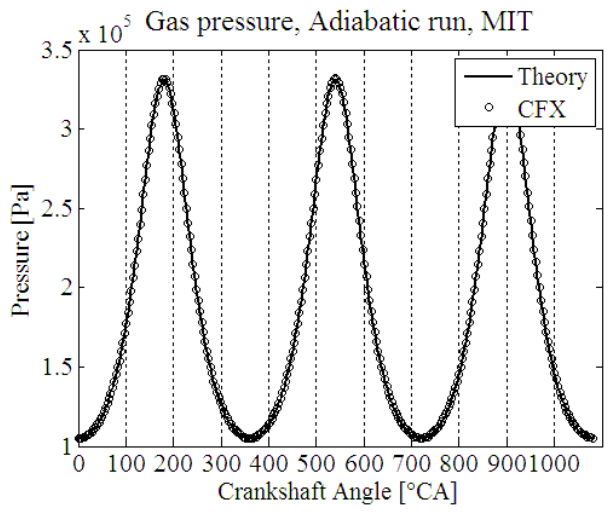

Figure 4.13: Adiabatic simulation and the thermodynamic analogy for the MIT $r=2$ model

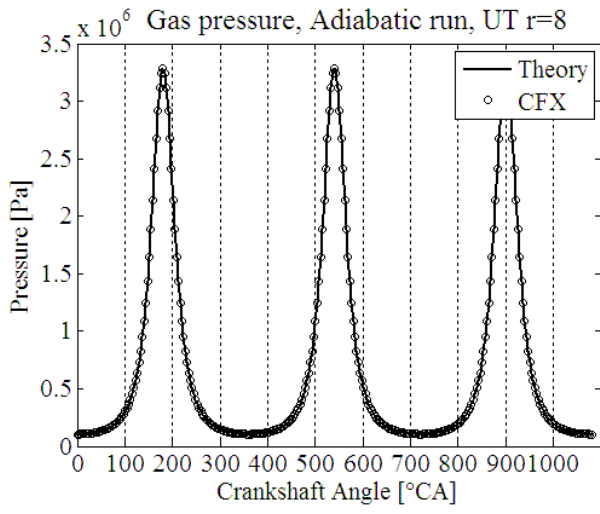

Figure 4.14: Adiabatic simulation and the thermodynamic analogy for the UT $r=8$ model 
Figure 4.I4, where the compression processes for two representative cases are displayed; one is the MIT model (compression ratio 2) and second the UT compression ratio 8 model ${ }^{27}$. The adiabatic models perform perfectly, and for two displayed, significantly different geometries, with meshes optimized through the earlier stated procedures, they are fully energy and mass conserving at every instance of a cycle.

This being confirmed, the adiabatic compression was not of interest for further research. Non-adiabatic cases will be discussed in the following paragraphs, as these could be validated on experimental measurements, and are in the central scope of this thesis.

\subsubsection{MIT Setup}

Simulations for the MIT model are compared to and validated on the experimentally obtained results, produced by A.A.Kornhauser during his $\mathrm{PhD}$ research on the same phenomena [2]. The geometry of the numerical models is created with respect to the real experimental setup and an overview of its dimensions is given earlier in this chapter in Table 4.I. Overview of the initial conditions for the MIT simulations is given in Table $4.3^{28}$. The temperature of all the surrounding walls is adopted constant, as reported in Kornhauser's thesis [2]. Gas spring walls in the MIT setup were cooled and maintained at a constant temperature.

\begin{tabular}{cccc}
\hline $\begin{array}{c}\text { Initial } \\
\text { conditions }\end{array}$ & $\begin{array}{c}\mathrm{P}_{\text {gas ini }} \\
{[\mathrm{Pa}] \cdot \mathrm{IO}^{5}}\end{array}$ & $\mathrm{~T}_{\text {gas,ini }}[\mathrm{K}]$ & $\mathrm{T}_{\text {walls }}[\mathrm{K}]$ \\
\hline \hline 2 RPM & 0.77757 & 295.3 & 295 \\
IOOO RPM & I0.4859 & 245.4 & 295 \\
\hline
\end{tabular}

Table 4.3: Initial conditions for the MIT models

\section{RPM}

After performing the numerical simulations, the results were post-processed and appropriately presented using CFX Post engine and Matlab. As described in the previous

\footnotetext{
27 Operating frequency is irrelevant for the adiabatic compression.

${ }^{28}$ Kornhauser [2] used revolutions per minute - [RPM] as notation for the operating frequency. Since the reference to all UT experiments is more convenient in $[\mathrm{Hz}]$, considerations for the UT experiments and simulations will be presented in this thesis with [Hz], and for the MIT setup with [RPM] notation.
} 


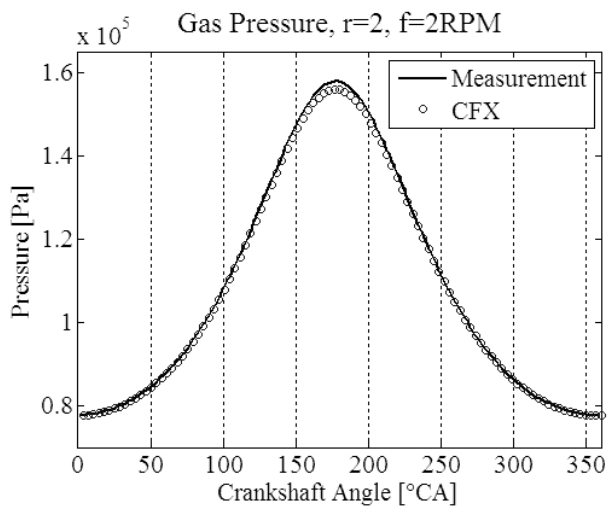

Figure 4.15: Measured and simulated gas pressure for the slow run, $r=2$

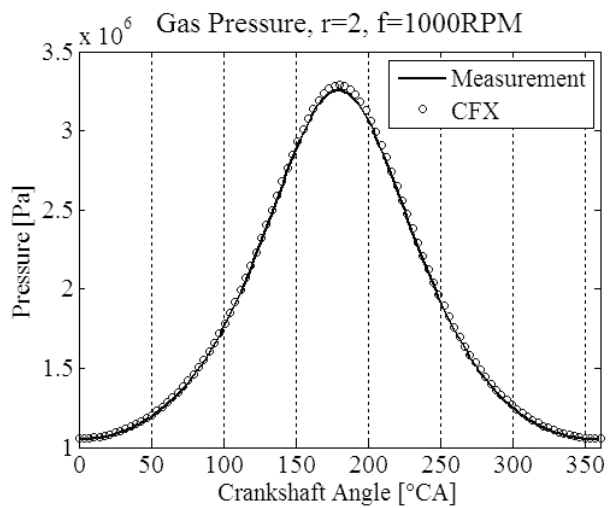

Figure 4.17: Measured and simulated gas pressure for the fast run, $r=2$

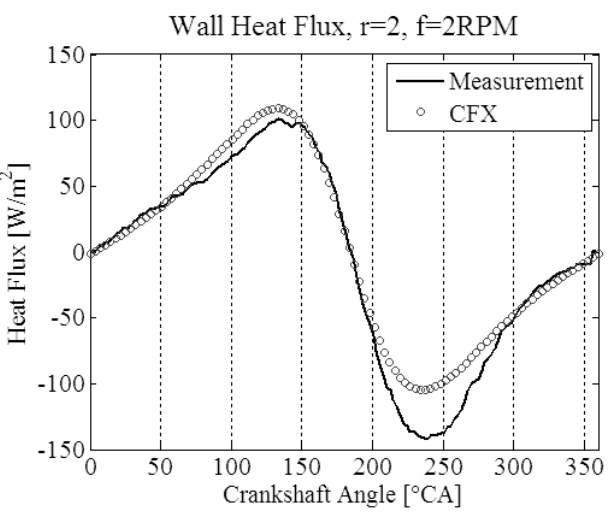

Figure 4.16: Measured and simulated wall heat flux for the slow run, $r=2$

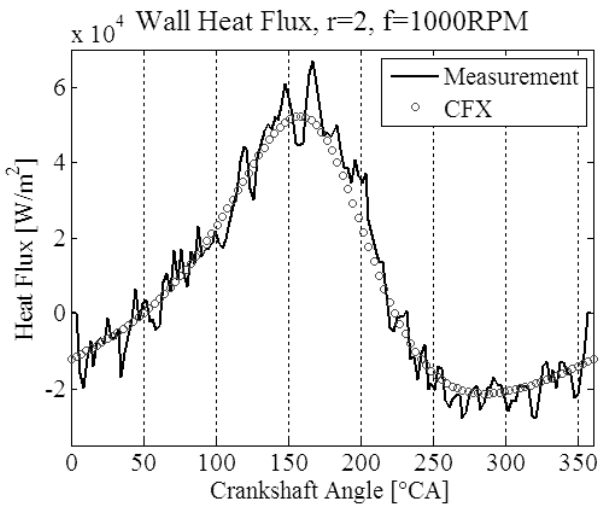

Figure 4.18: Measured and simulated wall heat flux for the fast run, $r=2$

paragraphs, a sufficient number of simulations was performed to ensure that the change of the mean observed properties was no more than $\mathrm{I} \%$ per cycle. After that a representative cycle was isolated, and the volume-averaged gas pressure and area-averaged wall heat flux were exported in function of time to comma-separated-values - .csv files. The time was then in Matlab translated to the angular position of the crankshaft, and simulated values were plotted against the experimentally measured data in representative figures. Results of the experiments and numerical simulations for compression ratio 2 and 2 RPM $(0.03 \mathrm{~Hz})$ runs are presented in Figure 4.I5 and Figure 4.I6.

As can be seen in Figure 4.I5, the numerically simulated pressure approaches very closely the experimentally obtained values. The pressure is oscillating between approximately 78 
$\mathrm{kPa}$ and $\mathrm{I} 60 \mathrm{kPa}$. The simulated pressure at the TDC is slightly lower than the measured pressure and the under-prediction accounts to $2.44 \%$ of the cyclic pressure amplitude. The phase of the measured pressure is perfectly followed though. It is interesting to notice that the peak pressure precedes the adiabatic pressure wave (peak at I $80{ }^{\circ} \mathrm{CA}$ ), but by no more than $2{ }^{\circ} \mathrm{CA}$. This is further discussed in Chapter 5 and Chapter 6.

Figure 4.I6 shows the measured and simulated wall heat flux. Heat transfer is well predicted for the $\mathrm{O}-2 \mathrm{IO}{ }^{\circ} \mathrm{CA}$ and $300-360{ }^{\circ} \mathrm{CA}$ ranges. It is positive in the compression phase of the cycle, positive being the heat transfer from the gas to the cylinder walls. Past the top-dead-centre, the expansion stroke starts and the heat flux becomes negative, with a negative maximum at approximately $235{ }^{\circ} \mathrm{CA}$. The numerical model under-predicts the reported measured values at this part of the cycle; the maximum negative heat flux is underestimated by almost $30 \%$. Some uncertainty about the measurements arises at this point, as from the graph it appears that more heat is being cyclically received than released by the compressed gas. The MIT gas spring is reported to be in the steady-state during the experiments [2], and the gas spring would have to actually produce work to balance this received heat and retain the zero-cycle-energy steady state equilibrium, while the mean compressed-gas temperature is actually slightly higher than the wall temperature as reported in the measurement database, bringing the process to be thermodynamically impossible. It is suspected that this discrepancy comes from the deviation of the projected piston trajectory and a small phase lag between the actual pressure and shaft position measurements, due to the motor loading at low speeds (also reported by Kornhauser).

\section{I000 RPM}

Results for the gas pressure and wall heat flux are presented in Figure 4.I7 and Figure 4.I8. The procedure for setting up and analyzing the numerical simulations as for 2 RPM runs is followed here, with initial and boundary conditions stated in Table 4.3. The measured pressure on Figure 4.I7 is observed to vary between I MPa and 3.3 MPa, with the maximum pressure again preceding the top-dead-centre, approximately at the same $\sim \mathrm{I}^{\circ}{ }^{\circ} \mathrm{CA}$. This is numerically reproduced with an over-prediction of $\mathrm{I} .4 \mathrm{I} \%$ of the pressure amplitude, but with a good match in phase.

The wall heat flux is plotted in Figure 4.I8; the amplitude varies from $\sim 50 \mathrm{~kW} / \mathrm{m}^{2}$ to $\sim-2 \mathrm{O}$ $\mathrm{kW} / \mathrm{m}^{2}$, with the peak heat flux in the compression stroke at approximately $\mathrm{I} 55^{\circ} \mathrm{CA}$. The instability of the measured heat flux is suspected to stem from the noise in the measured pressure signal. The wall heat flux is evaluated according to the described method (section 4.5.I and Appendix B). Still, the trend of the measurements, and both phasing and amplitude are clearly very well captured by the CFD model for the higher operating frequency. 


\section{Summary}

The results of simulations of the experiments done by A.A.Kornhauser [2] are presented in the above paragraphs. Two typical cases are investigated: slow (2 RPM) and fast (IOOO RPM) runs. Numerical results for the absolute pressure and wall heat flux are obtained by post processing according to the described method and compared to the experimentally measured values. The numerical models perform very satisfactory and the predicted values are matching the experiments well in both amplitude and phase.

\subsubsection{UT Setup - Compression Ratio 2}

The investigated range of operational conditions for the UT setup is much broader and comprises two compression ratios -2 and 8 , a spectrum of operating frequencies -2 to 25 $\mathrm{Hz}$ (I2O to I5OO RPM), and different initial charge pressures for compression ratio 2 experiments. Presentation of these results is thus sectioned by the compression ratios, each section covering the range of operating frequencies that are investigated.

Dimensions of the modelled domains for both compression ratios are presented earlier in Table 4.I, whereas the experimental machine design specifics are, together with the drawings of the crucial parts, sensing equipment specifics and the calibration procedure, described in details in Chapter 2 and Appendix C. An overview of the initial and boundary conditions for the simulations of experiments with the compression ratio 2 setup is given

\begin{tabular}{cccccc}
\hline $\begin{array}{c}\text { Initial } \\
\text { conditions, } \\
\mathbf{r = 2}\end{array}$ & $\begin{array}{c}\mathrm{P}_{\text {gas,ini }} \\
{[\mathrm{Pa}] \cdot \mathrm{IO}^{2}}\end{array}$ & $\mathrm{~T}_{\text {gas,ini }}[\mathrm{K}]$ & $\mathrm{T}_{\text {Head }}[\mathrm{K}]$ & $\mathrm{T}_{\text {Cylinder }}[\mathrm{K}]$ & $\mathrm{T}_{\text {Piston }}[\mathrm{K}]$ \\
\hline \hline $\mathrm{Hz}$ & $\mathrm{I} .06 \mathrm{I}$ & 272.7 & 298.8 & 298.8 & $298 . \mathrm{I}$ \\
$5 \mathrm{~Hz}$ & $\mathrm{I} .024$ & 269.9 & 299.3 & 299.4 & 298.5 \\
$\mathrm{IO} \mathrm{Hz}$ & 0.998 & 266.4 & 300.7 & 300.9 & 299.7 \\
$\mathrm{I} 5 \mathrm{~Hz}$ & 0.938 & 254.3 & 301.6 & 301.8 & 300.7 \\
$20 \mathrm{~Hz}$ & 0.936 & 253.8 & 302.5 & 302.8 & 301.8 \\
$25 \mathrm{~Hz}$ & 0.932 & 252.2 & 303.4 & 303.7 & 302.8 \\
\hline
\end{tabular}

Table 4.4. Initial conditions for the UT models, compression ratio 2 
in Table 4.4, and results of measurements and simulated runs are presented in Figure 4.I9 to Figure 4.23. As in the MIT considerations, initial and boundary conditions for the simulations are obtained as steady-state measured values from the respective experiments, and controlled parameters for the comparison between the experimental measurements and numerical results are the compressed gas pressure and wall heat flux.

\section{Gas Pressure}

This section presents the pressure measurements of the performed experiments and the results of respective numerical simulations for the investigated range of operating frequencies and the base helium charge. The pressure signals from sensing equipment are corrected for the calibrations determined prior to the experiments (as explained in Chapter 2) and plotted against the synchronously sampled crank angle. The numerically obtained pressure on the other hand is sampled as a function of time, and then correlated to the crank angle position. The obtained results are presented below.

Figure 4.I9 shows the results of the simulations, which were set-up as explained in the previous sections. All model boundaries are no-slip, solid walls, with imposed constant boundary conditions and a moving piston surface. Helium is specified as the ideal gas, undergoing a compression process imposed by the piston and exchanging heat with the surrounding walls.

The simulated pressures in Figure 4.I9 are observed to come close to the measured values, matching the measured compression curve closely but not entirely, especially for the slower runs $(2-\mathrm{I} 5 \mathrm{~Hz})$. The simulated pressure curve is slightly lagging behind the measured pressure, resulting in a small phase shift error (I. ${ }^{\circ} \mathrm{CA}$ for the $2 \mathrm{~Hz}$ run). The pressure is also over-predicted for the slower runs, and at the compression end it results in $\sim 8 \%$ higher pressure amplitude for the $2 \mathrm{~Hz}$ operating frequency. Interestingly, both phenomena seem to vanish with increasing operating frequency. This inconsistency required further research and resulted in the following.

As explained in Chapter 2, in the construction of the UT setup there are no piston rings to seal the gap between the compressing piston sides and cylinder wall. This unavoidably leads to the leakage of a very thin and diffusive gas such as helium. With the piston undergoing the compression stroke, the pressure above it rises and at the compression end becomes significantly higher than the pressure on the other side of the compressing piston: the volume here termed the buffer space. This pressure difference is a continuous potential for the equalization of pressures, and the related mass exchange between the two volumes. The pressure in the buffer space oscillates slightly as well, and is, due to the synchronized motion of the guiding and compressing piston and the geometry of the volume contained between them, in phase with the 'operating' compression (Figure 2.7), 

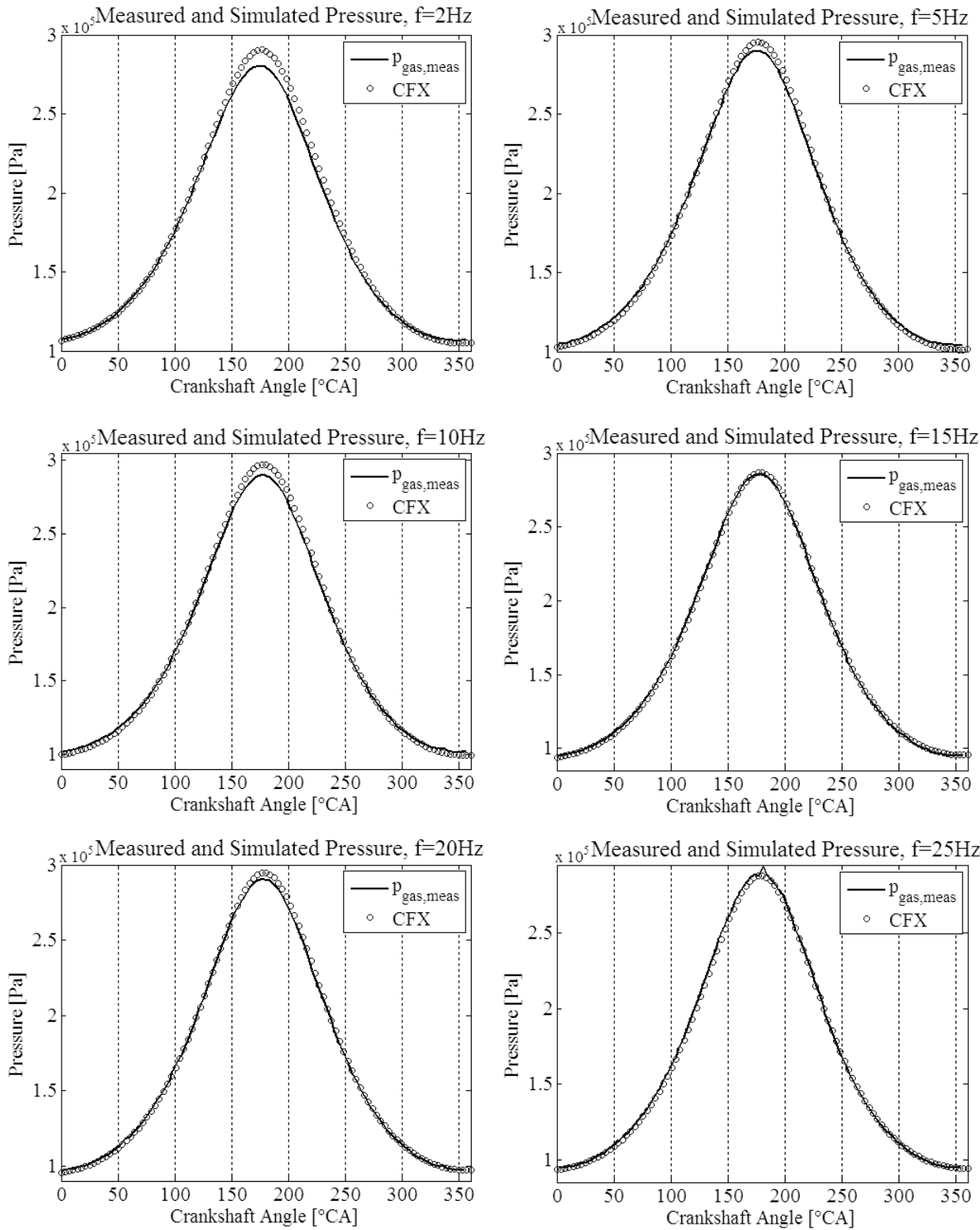

Figure 4.19: Experimentally measured and numerically simulated gas pressure, $r=2$ UT setup 


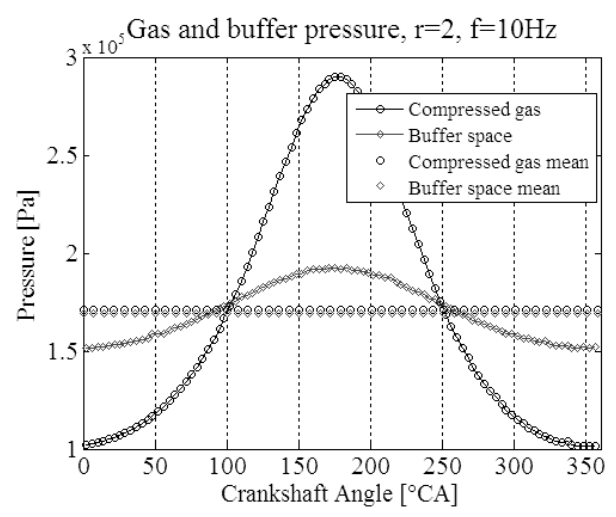

Figure 4.20: Experimentally measured pressure in the compression and buffer space, $r=2 U T$ setup, $f=10 \mathrm{~Hz}$

but with a much smaller amplitude. The compression ratio for the buffer space is approximately I.2 (Figure 4.20). After long enough operation and having reached the steady state, the cycle-mean pressures above and below the piston will equalize (dotted lines for two pressures almost on top of another in Figure 4.20). In the first half of the compression cycle, during the steady state operation, the pressure potential is firstly negative - helium flows from the buffer space to the compressed area, then after approximately half the stroke $\left(\sim \mathrm{IO}_{5}^{\circ} \mathrm{CA}\right)$, over the TDC towards half of the expansion stroke, it is positive - gas leaks from the compression space, and finally closing the cycle at $\mathrm{BDC}$ with negative pressure difference again. This phenomenon will reduce the pressure amplitude, as compared to the ideally sealed construction, since the mass is no longer conserved and at parts of the cycle less helium is being compressed in the experiment. Contrary to this, the numerical models presented in Figure 4.I9 simulate this ideal case, i.e. ensure the mass conservation in every time-step and thus over the whole operating cycle. This effect has the largest impact for the slower runs, which is in accordance with the theoretical expectations; this is further elaborated on in the following paragraphs.

\section{Modelling the Mass Transfer}

This leakage phenomenon is modelled by introducing an artificial mass source (sink). The mass source is created at the edge of the piston boundary, with the mass flux proportional to the expected leading parameters: the instantaneous pressure difference and constant length and width of the gap, according to the laminar flow theory, assuming a combined Hagen-Poiseuille and Couette flow [Io], [28]: 


$$
\dot{m}(t)=\pi D_{c y l} \rho(t)\left(R^{\prime 3} \frac{p_{g a s}(\mathrm{t})-p_{\text {buff }}(\mathrm{t})}{12 \mu L}-V_{P}(\mathrm{t}) \frac{R^{\prime}}{2}\right)
$$

$\dot{m}$ here is the instantaneous mass flow from the compressed volume to the buffer space, $D_{c y l}$ is the inner cylinder diameter, $R^{\prime}$ is the radial clearance between the piston and cylinder in the physical setup, and $L$ is the length of the axial piston-cylinder contact. $\mu$ is the gas dynamic viscosity and $V_{P}$ the instant velocity of the piston, positive in the compression stroke. The last factor, mass displaced by the piston can be neglected, as it is of low magnitude compared to the pressure induced transfer. The density of the gas is directly influencing the leakage, and it is here implemented as a transient value calculated in the model through the conservation equations. Leakage is localized at the edge of the piston by using a Heaviside step function in the CFX Expression Language (CEL):

$$
\dot{m}=\dot{m} \cdot \operatorname{step}\left(\sqrt{x^{2}+z^{2}}-\frac{D_{\text {Pist }}}{2}\right)
$$

where $x$ and $z$ are radial coordinates in the Cartesian coordinate system, $y$ being the axial component. The Heaviside (unit) step function's value returns zero for a negative argument, one for a positive argument and $1 / 2$ if the argument equals zero.

The typically simulated mass oscillation of the captured gas during one cycle, resulting from this model is shown in Figure 4.2I. The mass oscillates according to the pressure potential on two sides of the piston-cylinder contact, and its grows in the first part of the cycle, up to $\sim \mathrm{IOO}{ }^{\circ} \mathrm{CA}$, with the gas leaking from the buffer space to the compressed volume. Mass flux then changes the sign, becomes positive and the gas leaks from the compressed domain. After $\sim 24 \mathrm{O}^{\circ} \mathrm{CA}$, the compressed gas pressure again drops below the

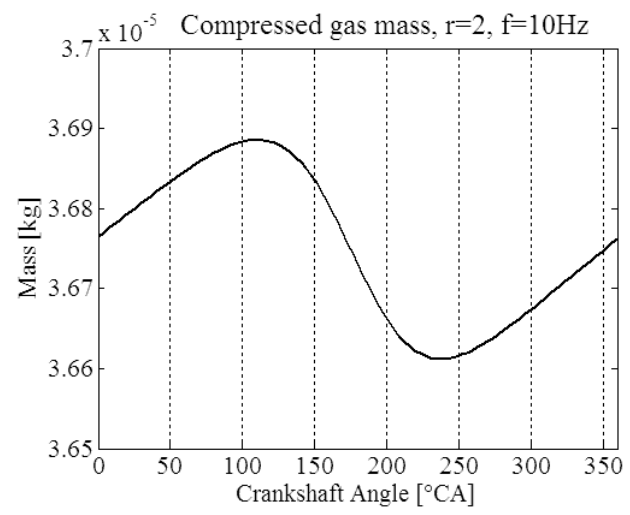

Figure 4.21: Oscillation in the mass of the compressed gas, $r=2$ UT setup, $f=10 \mathrm{~Hz}$ 


\begin{tabular}{ccccccc}
\hline $\begin{array}{c}\text { Displaced } \\
\text { mass } \\
\text { amplitude }\end{array}$ & $2 \mathrm{~Hz}$ & $5 \mathrm{~Hz}$ & IO Hz & I5 Hz & $20 \mathrm{~Hz}$ & $25 \mathrm{~Hz}$ \\
\hline \hline $\mathbf{k g}$ & $6.063 \mathrm{E}-07$ & $2.6 \mathrm{IIE}-07$ & $\mathrm{I} .34 \mathrm{OE}-07$ & $8.365 \mathrm{E}-08$ & $6.9 \mathrm{I} 7 \mathrm{E}-08$ & $\begin{array}{c}5 . \mathrm{I} 78 \mathrm{E}- \\
08\end{array}$ \\
$\begin{array}{c}\% \text { mean } \\
\text { mass }\end{array}$ & I.59I & 0.70I & 0.365 & $0.23 \mathrm{I}$ & 0.187 & 0.145 \\
\hline
\end{tabular}

Table 4.5: Amplitude of the mass oscillation during one compression cycle, UT setup $r=2$

pressure in the buffer space, and the gas leaks upwards. The maximum amplitude of the mass exchange is here calculated to be approximately $0.4 \%$ of the contained mass.

The magnitudes of the displaced helium mass, obtained in all the simulations that will be discussed in the following paragraphs, are stated in Table 4.5. It is interesting to observe the percentage of the operating fluid being cyclically displaced for the range of operating speeds. The rate of the mass displacement is the highest for the slowest run, and the amplitude of the maximum mass exchange amounts to $\sim$ I. $6 \%$ of the cycle mean mass of the operating gas, while for the fastest runs it is lower by approximately an order of magnitude. This stems from the dynamics of the process; leakage occurs during a much longer period of time for the slower processes, with comparable pressure differences. A linear correlation between the compression cycle duration and the mass leakage rate is disturbed by the higher pressure amplitudes for the higher operating frequencies (as the process approaches isentropic compression). The buffer space pressure amplitude does not strongly depend on the frequency but only on the initial gas charge, which is approximately the same for all compression ratio 2 runs, and thus the pressure-difference potential for the gas leakage comes dependent only on the process happening above the piston.

Further, from Equation (4.4) it is obvious that the radial clearance $R^{\prime}$ and the pistoncylinder contact length $L$ are strongly influential parameters for the gas leakage rate. Both parameters can be directly determined from the piston and cylinder geometries (Chapter 2), but their effective values during a compression cycle are very discussible. There are several degrees of freedom:

Being that the piston is profiled at its contact surface to the cylinder, the effective contact length here is not straightforward to determine. The influence of radial grooves (Figure 2.3 a)) is reported to be beneficial to the reduction of gas leak, $[\boldsymbol{I}]$ and [29], but this was not found to be precisely quantified. On the other hand, both the contact length, and especially effective radial clearance are sensitive to a possible inclination of the compressing piston. The compressing piston lies on a very small diameter horizontal surface of the connecting rod, with additional thin rubber cylinders placed at the contacts 
on both ends of the rod (constructed for the flexibility and the reduction of contact forces in the case of axial misalignment of the piston and cylinder). This hardly assures the strictly central positioning of the piston. Lastly, the compressing piston built in at this stage of the project is made of soft bronze that was imposed to wear-off during the course of experiments $^{29}$. All of this highly hampers a good definition of projected dimensions.

Nonetheless, after the introduction of the mass source at the piston as described above, the initial model was created on basis of the machined dimensions of the compression domain, thus with the gas domain diameter equal to the inner diameter of the installed cylinder $\left(D_{g l l}=50.2 \mathrm{I} 3 \mathrm{~mm}\right)$, the mean radial clearance of $\mathrm{I} 6.5 \mu \mathrm{m}$, and the contact length of I $5 \mathrm{~mm}$. This model performed very well, with the over-prediction of pressure at the compression end of only $2.5 \%$ for the slowest runs, as compared to the $8 \%$ for the model with leakage not modelled. The results for the higher operating frequencies were less affected by the leakage even prior to the introduction of the mass source, as already discussed, and the imposed errors for faster runs are ever smaller. Still, having in mind the above stated degrees of freedom of the model, the radial clearance was gradually increased to meet the exact match for the measured pressure. This correction came as the first choice and most obvious, since the experimental piston was actually expected to have physically worn out and reduced its diameter. The radial clearance was increased to I 9.5 $\mu \mathrm{m}$, and the obtained results of this model are presented in Figure 4.22.

With the described method implemented in the previous model, results came much closer to the measured values. On Figure 4.22 one can see the clear improvement for the gas pressure results, most obvious for the slower runs. Errors are eliminated both in amplitude and phase, and the whole range of operating frequencies is properly simulated and very closely matches the measured values. The largest error is here for the simulation of the IO $\mathrm{Hz}$ experiment and amounts to I.9 \% of over-prediction. The presented method can be adopted as very effective, and increases the simulation potential even for the non massconserving construction of the compression machines such as the UT setup.

\section{Wall Heat Flux}

In section 4.5.2, in the MIT setup considerations, the surface averaged wall heat flux was found to be predictable to a high extent. It was explained that both measured and simulated heat flux are directly dependant on the piston work and the internal energy of the compressed medium at every time instance of the cycle. With this in view, a precise determination of the mass of the fluid inside the compressed volume becomes crucial for the performance of predicting models and accuracy of the evaluated heat flux.

\footnotetext{
29 Piston diameter at the end of experiments was not precisely measured.
} 

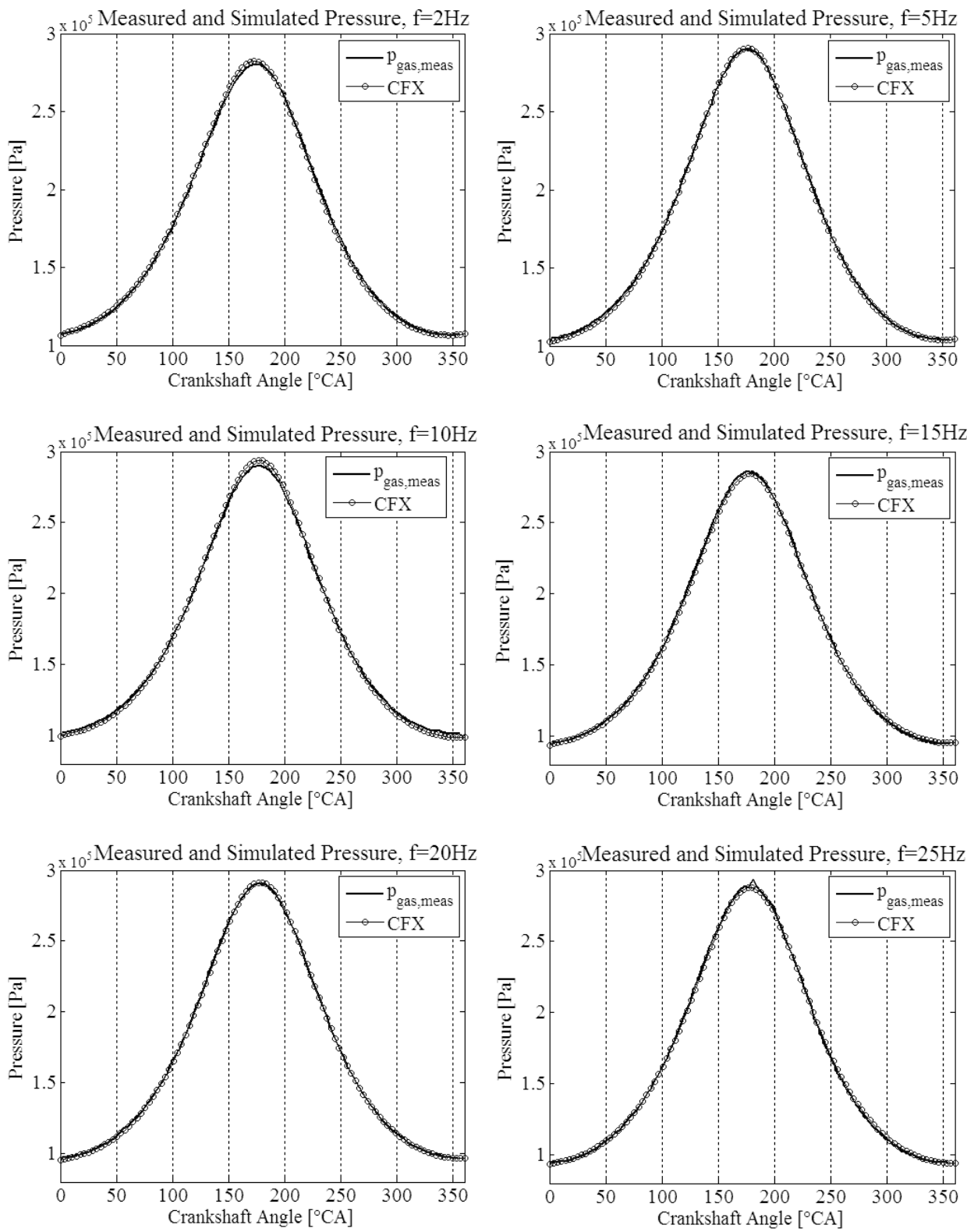

Figure 4.22: Experimentally measured and numerically simulated gas pressure with the modelled gas blow-by, $r=2$ UT setup 

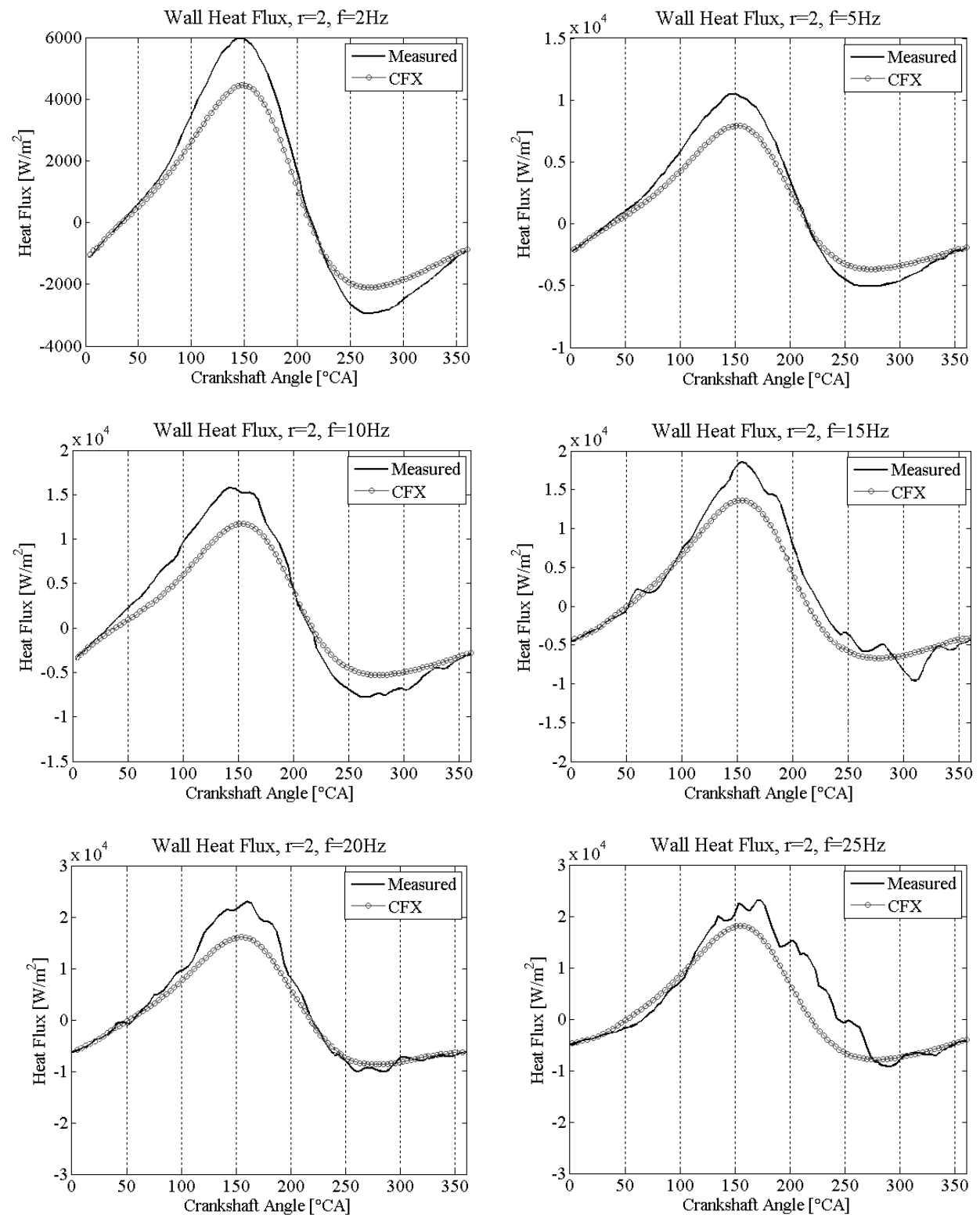

Figure 4.23: Calculated and simulated wall heat flux with the modelled gas blow-by, $r=2$ UT setup 
In the MIT setup, the construction with the piston rings prevents the gas leakage from the compressed space, and the mass of the compressed gas can be considered to be constant from the initial charge moment prior to the operation, to any cycle reasonably later in the continuous operation during the experiment (and thus also along any particular compression cycle itself). In the paragraphs above, it was shown that in the UT setup the mass conservation cannot be ensured, and that the fluid is displaced from and to the compressed section during a compression cycle and especially over a longer time of machine operation, whereas the method presented for comparing the measured results for the wall heat flux is valid only when the compressed mass is constant. Otherwise, one has to precisely determine the instantaneous mass (or temperature) at every time step of the compression cycle, which is not viable in the present experimental setup. This hampers the validation of the numerical models with respect to the wall heat flux prediction for the UT setup, in the way it was done for the MIT experiments ${ }^{30}$.

Nevertheless, it has been shown in Figure 4.I9 and Figure 4.22 that with the faster operation, leakage becomes less influential on the amplitude of the pressure curve, as relatively less fluid is being transferred from and to the compression space. Thus it is interesting to explore to what extent this affects the wall heat flux calculated from the measured pressure signal and evaluated by the previously shown procedure. This is presented in Figure 4.23, for the experiments discussed with respect to the pressure results in Figure 4.22.

The heat flux curve marked 'measured' is, as earlier, calculated for the measured pressure of the gas, through the thermodynamic laws and mass estimated at the beginning of the compression cycle. The simulated curve at the other hand is the CFX output for the areaaveraged wall heat flux, obtained from the numerical models presented in the previous section, with the mass loss modelled. Having all above in mind, the results shown in Figure 4.23 are as expected. The change of the internal energy of the gas undergoing compression is directly proportional to its mass, and, being that the mass is displaced out of the compression space during the compression stroke, an error in the increase of internal energy of the compressed gas is in this way directly introduced. The estimated heat transfer from the compressed gas to the surrounding walls has to be reduced by the amount of energy lost with the displaced gas. On the way back, energy is artificially restored by the gas returning from the buffer to the compression space, and thus again virtually less energy gets to be transferred from the walls back to the gas in order to satisfy the balance of the model. This proves the assumption that the described method for determining the average wall heat flux is applicable only when the mass conservation

\footnotetext{
30 Nevertheless, this was indirectly attended in Chapter 6, where the simulations for both MIT and UT geometries and a range of operating frequencies were evaluated on a set of analytical correlations.
} 
during the cycle is ensured. As with the pressure considerations, and in accordance to Table 4.5, with relatively less mass of the energy carrying gas being displaced also less energy will be lost, and the above stated effects are for the higher operating frequencies not as important.

\section{Increased Charge Pressures}

Higher helium charge pressures were also investigated with the compression ratio 2 UT gas spring and these pressure measurements and the respective simulations are summarized and presented in Appendix D. Results of these experiments conform to the previous observations for lower operating frequencies, whereas as the charge pressure and operating frequencies increase, the (suspected) effects of the secondary motion and/or tilting of the piston come into place and distort the predictions based on the baseline clearance of $\mathrm{I} 9.5 \mu \mathrm{m}$. These effects are also observed and discussed in the next section, for the experiments with the compression ratio 8 gas spring.

\subsubsection{UT Setup - Compression Ratio 8}

The experiments for the compression ratio 8 gas spring ${ }^{3 \mathrm{I}}$ were pursued following the same routine as described for the compression ratio 2. As already shown, the geometry of the $r=8$ gas spring is different from $r=2$ only in the height of the compression head, with the piston and cylinder geometries and the stroke of the machine kept the same as for the previous experiments. The investigated sets of operating conditions are also taken similar; the experimental gas spring was charged at room temperature to the selected pressures, and operation was started. Measurements were recorded in steady state operation, and then initial and boundary conditions for the numerical simulations were extracted from recorded representative cycles. Wall temperatures, gas pressure and crankshaft angle were directly measured, and the gas temperature was estimated using the procedure described in subchapter 4.4. An overview of the boundary and initial conditions for compression ratio 8 is given in Table 4.6. Results of the numerical simulations compared to the measurements from the respective experiments are presented in Figure 4.24 to Figure 4.27 .

\footnotetext{
${ }^{31}$ Due to the necessary connections for the sensors in the compression head, several 'dead' volumes had to be introduced in the $r=8$ compression setup. For this reason, compression ratio is in reality somewhat lower, and with all added gas connection lines and valve and sensor dead volumes taken into account, amounts to $7 \cdot 75$. Still, for the sake of simplicity of reference, these considerations will be referred to as compression ratio 8 .
} 


\begin{tabular}{cccccc}
\hline $\begin{array}{c}\text { Initial } \\
\text { conditions }\end{array}$ & $\mathrm{P}_{\text {gas }}[\mathrm{Pa}] \cdot \mathrm{IO}^{5}$ & $\mathrm{~T}_{\text {gas }}[\mathrm{K}]$ & $\mathrm{T}_{\text {Head }}[\mathrm{K}]$ & $\mathrm{T}_{\text {Cylinder }}[\mathrm{K}]$ & $\mathrm{T}_{\text {Piston }}[\mathrm{K}]$ \\
\hline \hline $2 \mathrm{~Hz}$ & $0.42 \mathrm{I}$ & 279.5 & 294.0 & 298.8 & 294.2 \\
$5 \mathrm{~Hz}$ & $0.4 \mathrm{O} 4$ & 266.9 & 295.4 & 294.5 & $295 . \mathrm{I}$ \\
$\mathrm{IO} \mathrm{Hz}$ & 0.330 & 257.7 & 297.0 & 296.0 & 296.2 \\
$\mathrm{I} 5 \mathrm{~Hz}$ & 0.272 & $2 \mathrm{I} 2.0$ & $299 . \mathrm{I}$ & 297.8 & 297.6 \\
$20 \mathrm{~Hz}$ & 0.250 & $\mathrm{I} 89.7$ & $301 . \mathrm{I}$ & 299.3 & 299.0 \\
$25 \mathrm{~Hz}$ & 0.230 & $\mathrm{I} 63.2$ & 303.0 & 300.9 & 300.5 \\
\hline
\end{tabular}

Table 4.6: Initial conditions for UT models, compression ratio 8

\section{Gas Pressure}

After conducting the described experiments, settings for the initial simulations for the compression ratio 8 were based on the conclusions from $r=2$ considerations. The performance of the simulations neglecting the compression gap leakage was also tested and proven to lead to even larger errors: simulated pressure was over-predicted by as much as $32 \%$ for the $2 \mathrm{~Hz}$ run, with a significant over-prediction of $7.4 \%$ even for the fastest $-25 \mathrm{~Hz}$ run. The next step was thus the creation of the analogous model with the mass transfer incorporated, and the settings for the $\mathrm{r}=2$ model were directly imported: the clearance gap was again set to I $9.5 \mu \mathrm{m}$ and the contact length to $\mathrm{I} 5 \mathrm{~mm}$. Results are also processed in the same way and are presented in Figure 4.24.

Observing Figure 4.24, it is obvious that the numerical model for compression ratio 8 does not perform nearly as well as for $r=2$. The disagreement is obvious through the entire range of operating frequencies, with an added error in the phasing of the pressure curve, especially visible for the slower runs. Because the compression is here underestimated in the simulations, and that this error reduces with the increase of the operating frequency, this leads to the conclusion that the gap between the piston and cylinder, and the associated leakage rate, were this time modelled too large. Logical next step was the investigation of the effect of reducing the radial clearance on the simulation results, and this was systematically done until the errors came within acceptable limits $(4 \mu \mathrm{m}$, discussed in the following paragraphs). 

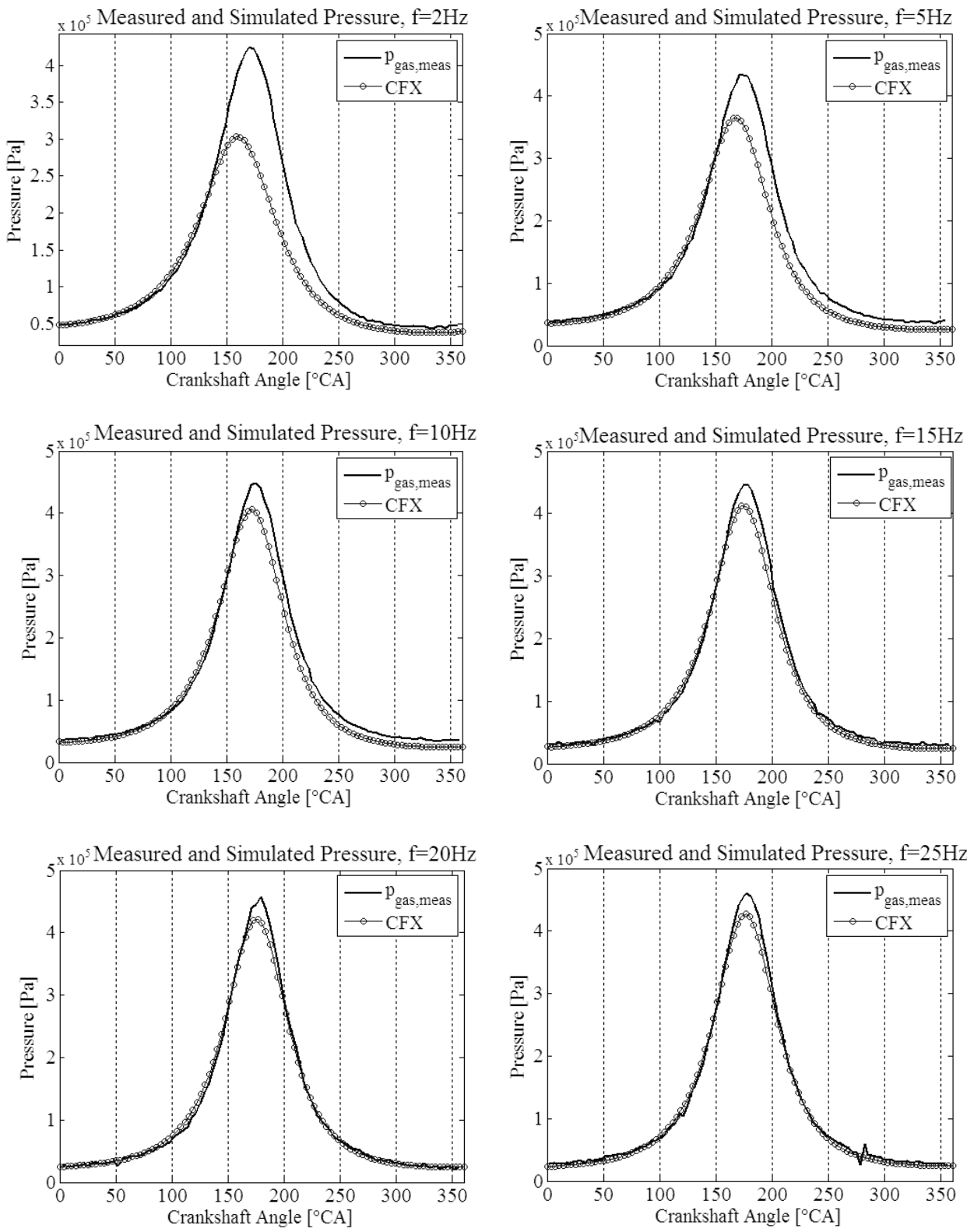

Figure 4.24: Experimentally measured and numerically simulated gas pressure, $r=8$ UT setup, with the modelled radial clearance $19.5 \mathrm{\mu m}$ (as for $r=2$ ) 


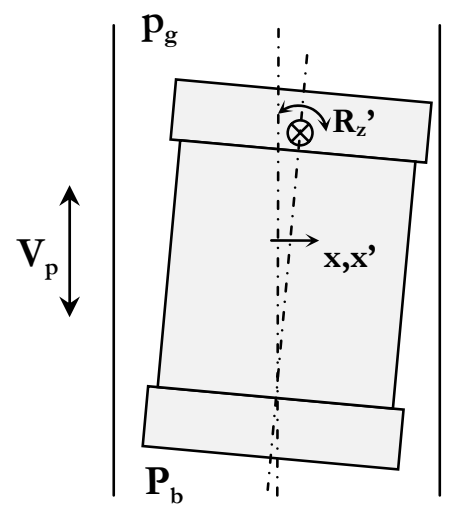

Figure 4.25: Secondary motion and misalignment of the piston

Reasons for this inconsistency with the conclusions for compression ratio 2 were investigated. There are two effects that could have contributed to this phenomenon.

Namely, the experimental setup is installed according to the assembling procedure described in Appendix C. The cylinder liner was hand-slid on the piston standing on the partially flexible connecting rod. It is well possible that, during the mounting, the piston rod got slightly bent in one of the two necks, and the piston got initially transversely displaced (Figure 4.25, $\mathrm{x}$ to $\mathrm{x}^{\prime}$ ), and/or, even more favourable to this phenomenon, rotated in the $x-y$ plane $\left(R_{z}{ }^{\prime}\right)$. Further, with the increase of the pressure amplitude of the compression cycle with the higher compression ratio, the maximum forces acting on the piston top surface also increase. By using the flexible necks on two sides of the piston rods, the piston is allowed to re-position in the cylinder and incline in the vertical plane in the tolerance of this clearance. Forces acting on the piston during compression will support this movement; once the secondary displacement of the piston gets initiated, they will not return the piston to its ideal position, but rather add to this instability and make the piston further incline towards the cylinder and possibly finally slide on its edges against the walls. With the higher pressure acting on the top surface, forces on it become stronger than the side forces originating from the pressure in the thin gas film on the piston sides, and the piston rotates around the horizontal axis, closing a part of the radial clearance. This was also observed for higher charge pressures with the compression ratio 2 gas spring and reported in Appendix D. In the same line, secondary motion of the piston was also examined in $[\boldsymbol{I}]$, and it was shown that for this piston design, radial gas film forces are too weak to stabilize the piston. At the start of the compression stroke the piston can be slightly inclined, and under the strong forces acting on the top surface of the piston, stays under this inclined angle through the rest of the stroke. The forces in case of compression ratio 8 become rapidly higher and incline the piston very early in the compression cycle. 

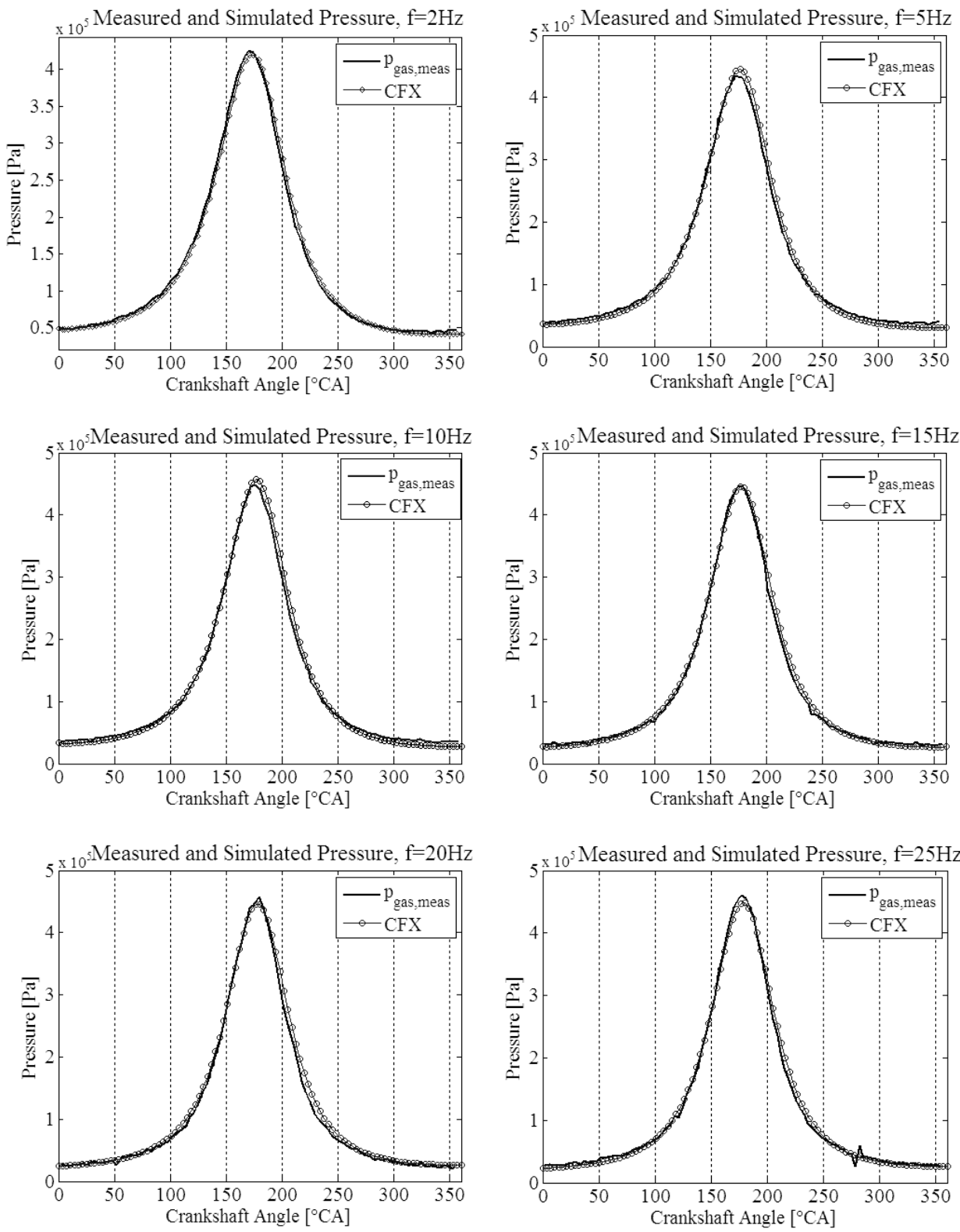

Figure 4.26: Experimentally measured and numerically simulated gas pressure, $r=8$ UT setup, with the modelled radial clearance $4 \mu \mathrm{m}$ 
The sensitivity of the gas leakage rate on the influence of the piton-cylinder clearance and the contact length was already mentioned in the previous section. With the piston inclination, the distance between the piston edge and cylinder wall at two opposite sides will reduce, while in the other, orthogonal direction, stays the same, provided there is no transverse displacement in this direction. Such inclination of the piston effectively closes the gap for the gas leakage, and the simulation model with I9.5 $\mu \mathrm{m}$ of radial clearance then overestimates the mass loss and compression is thus underestimated. Being that the accurate determination of the secondary motion of the piston is not in the scope of this thesis, an iterative approach in determining the effective radial clearance was again pursued to compensate for the exact angle of inclination and its effect to the gas leakage. Best results were obtained for the process modelled with approximately one fifth of the previously modelled radial piston clearance $-4 \mu \mathrm{m}$ and contact length kept the same. The results with this improvement are shown in Figure 4.26.

With the inclined piston, the shape of the gap allowing the leakage will be very different from a regular ring with a $4 \mu \mathrm{m}$ radial thickness, and thus this clearance will not be directly representative for the real experiment, but more of an effective parameter imposing the equivalent effects of the distorted radial clearance and contact length on the leakage rate. Results presented in Figure 4.26 are obviously significantly improved with this compensation, and the experimentally measured pressures are closely matched. The largest error is introduced in the $5 \mathrm{~Hz}$ simulation; pressure is overestimated by $2.7 \%$ of the cycle amplitude.

\section{Mass Conservation}

The mass oscillation for the compression ratio 8 gas spring with $4 \mu \mathrm{m}$ radial clearance is displayed in Table 4.7. The absolute amount of displaced gas is reduced by approximately $80 \%$ as compared to the compression ratio 2 results. This is in accordance with the suspected reduction in the leakage gaps, the radial clearance being the strongest influencing term in Equation (4.4). Gas property parameters in relation to leakage density and viscosity are similar to the values in compression ratio 2 experiments and do

\begin{tabular}{ccccccc}
\hline $\begin{array}{c}\text { Displaced } \\
\text { mass }\end{array}$ & $2 \mathrm{~Hz}$ & $5 \mathrm{~Hz}$ & IO Hz & I5 Hz & $20 \mathrm{~Hz}$ & $25 \mathrm{~Hz}$ \\
\hline \hline$\pm \mathrm{kg}$ & $\mathrm{I} .267 \mathrm{E}-07$ & $5.2 \mathrm{I} 9 \mathrm{E}-08$ & $2.526 \mathrm{E}-08$ & $\mathrm{I} .634 \mathrm{E}-08$ & $\mathrm{I} .255 \mathrm{E}-08$ & $\begin{array}{c}\text { I.084E- } \\
08\end{array}$ \\
$\begin{array}{c}\% \text { mean } \\
\text { mass }\end{array}$ & $\mathrm{I} .462$ & 0.669 & 0.350 & 0.226 & 0.169 & 0.136 \\
\hline
\end{tabular}

Table 4.7. Amplitude of the mass oscillation during one compression cycle, UT setup $r=8$ 

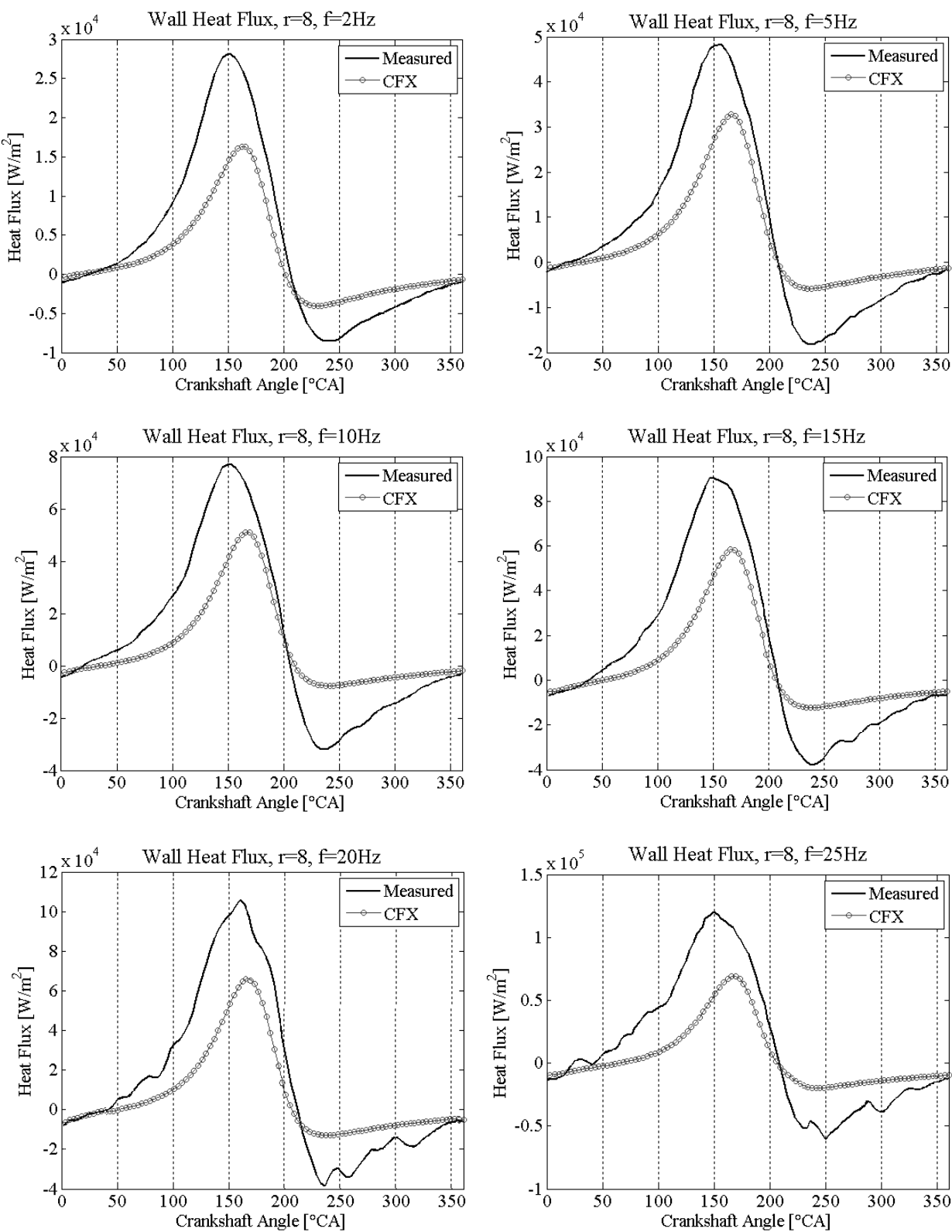

Figure 4.27: Calculated and simulated wall heat flux, $r=8$ UT setup, radial clearance $4 \mu \mathrm{m}$ 
not introduce additional effect on the leakage rates. Lastly, the pressure amplitude is expected to rise significantly, but it is re-adjusted with every compression time-step through the gas leakage. The percentage of the displaced fluid comes is in the similar range as for the compression ratio 2 , since less mass is captured in the operating volume.

\section{Wall Heat Flux}

Finally the results for the wall heat flux are discussed. The method for its indirect evaluation presented for the experimental machine used at MIT is already proven not to be valid for the UT setup with compression ratio 2 because of the mass imbalance during a compression cycle. Nevertheless, the compression ratio 8 runs were engaged as well, to check for the presented conclusions. Results of these considerations are shown in Figure 4.27, and conform to the observations for compression ratio 2. Numerical results deviate from the thermodynamically determined energy loss even more than for $r=2$. The heat transfer between the compressed gas and the surrounding solids is even more biased, and the necessity of mass conservation at every instance of compression cycle for the successful application of this method is once more obvious.

Experiments with higher charge pressures have not been performed for the compression ratio 8 setup, as these would not have an added value to this research at this stage because of the presented issues related to the compressed gas leakage.

\subsection{Summary}

Finite volume models of the investigated experimental gas springs, presented earlier in Chapter 2, are created in ANSYS CFXI I.O, their operation is simulated and the results are analyzed and compared to the experimental measurements performed at the Massachusetts Institute of Technology and the University of Twente. A measurement results database for the MIT experiments was obtained by courtesy of Professor Alan Abram Kornhauser and presents the experiments performed on the compression ratio 2 gas spring operating on two different compression frequencies ( 2 and IOOO RPM $\sim 0.03$ and $16.7 \mathrm{~Hz}$ ). Experimental database for the runs performed at the University of Twente is obtained during the course of the research presented in this thesis, and comprises a much broader set of parameters, including two compression ratios -2 and 8 , set of 6 operating frequencies $(2-25 \mathrm{~Hz})$ and different initial charge pressures.

Numerical models are presented in details in subchapter 4.I, starting from the geometry specifics of the modelled domains and their relations to the real experimental machines, the used meshing techniques and a discussion on the optimization steps of the spatial, and in subchapter 4.2 of the temporal discretization. A view on the numerical accuracy of the 
acquired results and the involved computational costs is given. This is followed in 4.3 by the description of the imposed boundary conditions to the models, boundary motion and mesh deformation modelling, and discussion on the initial conditions. The sets of initial conditions for the simulations are nevertheless presented at the beginning of every concerned section for the three investigated geometries (MIT compression ratio 2 and UT 2 and 8$)$.

In subchapter 4.4 a method is presented to estimate the gas temperatures and correlated mass of the captured gas in a gas spring during the experiment at positions close to BDC, and in this way enable numerical modelling when direct measurement of the instantaneous gas temperature is not possible to achieve. This is finally followed by a wide discussion of the results in subchapter 4.5 , with the computational procedure of the raw data presented in section 4.5.I, the results for the MIT setup in section 4.5.2, UT compression ratio 2 in section 4.5 .3 and UT compression ratio 8 in section 4.5.4. Evaluated parameters are the gas pressure and wall heat flux.

The results of the numerical simulations for the MIT experiments show to be very satisfactory. Gas pressure and wall heat flux are very well predicted in both amplitude and phase, numerical models perform very well, with the maximal imposed error in the pressure results of $2.44 \%$ of the gas pressure amplitude.

The simulations for the UT setup initiated extended considerations. Mass transfer model, from- and to the compressed section of the UT gas spring is introduced, being that the UT construction does not incorporate piston rings or other compression seals, and the gas leakage past the piston during compression is intrinsically partially allowed. The estimated mass transfer and the modelling method are presented in detail in section 4.5 .3 , together with the gas pressure results for the adjusted numerical model. Numerical models with the mass source modelled show a very significant improvement, and the largest error in the pressure amplitude on the corrected model amounts to I.9 \% of over-prediction for the baseline charge pressure. The heat flux correlation explained in section 4.5.4 Appendix B fails here, and the comparison between the modelled and experimental results for the wall heat flux cannot further be made, due to the lack of the cycle-mass-conservation precondition.

Analogous analysis was performed for the UT compression ratio 8 model, which (together with the results for increased charge pressures in the compression ratio 2 gas spring) gave insight into more phenomena involved in the compression-related processes in the investigated construction of a gas spring construction investigated here. Namely, due to the secondary piston motion and the absence of a well-controlled setup assembling procedure, the experimental piston was inclined during compression and the effective radial clearance had to be corrected (reduced). With the described correction, the modelled 
results followed the measured values very consistently for all operating frequencies, and the largest error for compression ratio 8 models was I.9 \% of the gas pressure amplitude.

ANSYS CFXII.o has proven to be a very robust and user-friendly finite volume numerical package for the purposes of research presented here. Created models showed the potential of the numerical modelling in the process evaluation and prediction of physical parameters of interest. Future work should concentrate on the development of a direct heat flux evaluation technique, which would allow for full validation of the models with a view on the spatial distribution of heat transfer, in addition to the area-averaged wall heat flux presented here. In parallel, work should be continued on the two-domain numerical models and simulations, extending the modelling potential to the thermal mapping of the surrounding solid walls and in-wall temperature distribution. Finally, the conclusions presented here will ideally be employed towards the modelling efforts in the modern oilfree compressor constructions, with the commercial designs and valve induced flows instead of closed compression. 


\section{CHAPTER THERMODYNAMICS AND FLUID FLOWS}

Quantitative analyses of thermodynamic properties, such as the gas pressure, temperature and heat loss, help understand and improve the performance of physical processes and mechanical constructions, clarify the limits of the requirements to be set before them, and extend the predictability of their behaviour. Deeper qualitative analyses to follow it, such as presented in this chapter, aim at gaining insights in mechanisms of physical phenomena and search for windows of improvement.

Having experimentally validated the numerical models on the preceding pages, thermodynamical and fluid flow analyses of the numerical and experimental results are pursued here. An attempt is made to add more general considerations, observations and conclusions to the so far presented, more specific work.

\section{I Introduction}

The wide range of physical and numerical experiments pursued in this research, some of which is presented in Chapter 4, invites for better understanding of occurring physical phenomena. On the following pages, several topics that intuitively attracted attention during research work are being discussed. These could serve in the scientific and engineering investigations, triggering the design and optimization paths, as well as a general observation summary. 
The work presented here strongly relies on the results of numerical work discussed to this point, and incorporates theoretical considerations met in general physics, thermodynamics and fluid mechanics. Subchapter 5.2 evaluates the described spatially discretization of the numerical models on the Kolmogorov scale, and presents the related considerations. In subchapter 5.3, a general discussion on investigated thermodynamical properties (gas pressure, temperature and the heat transfer) is summarized, with a comparison of the obtained relations with the existing thermodynamic laws. Finally, extended observations on the fluid flow patterns that develop in the observed gas spring geometries close these topics with subchapter 5.4. Investigations presented in this section are based on the experiments done on the MIT setup, since the validation data were available early in the project and most of this work was done prior to performing experiments with the UT setup. Nevertheless, based on the similarity of the investigated setup geometries, and encouraged by the performed cross-investigations on the translatability of conclusions between the models, a presumption is made that the conclusions made on the MIT setup can be generalized to other cases as well.

\subsection{Kolmogorov Scale}

In Chapter 4 the discretization routine of the modelled numerical domains, and the methodology of assurance of the simulation accuracy by re-meshing was presented. This was done by investigating the mesh size influence on the result parameters of interest. The intention here is to investigate to what extent the smallest fluid flow structures, according to the established turbulence theories, are taken into account in the presented simulations. A general introduction is given first, followed by a more specific discussion.

\subsection{General Concept}

The concept of the smallest energy scales in a developed turbulent flow is introduced by L.F. Richardson in I922 [30], in his concept of the energy cascade: "Big whorls have little whorls that feed on their velocity, and little whorls have lesser whorls and so on to viscosity". The idea behind is the following:

The largest eddies themselves are subject to inertial instabilities and rapidly break-down and dissolve into smaller vortices. The lifespan of a typical eddy is rather short, of the order of the so-called turn-over time, $l / u, l$ and $u$ being the length and velocity scale, respectively. Smaller eddies themselves are also unstable and they pass their energy onto even smaller structures and so on. Crucially, the whole process is driven by the inertial forces, and viscosity plays no part in this cascade for large eddies. The cascade comes to a halt, however, when the eddy size becomes so small that the Reynolds number based on 
the size of smallest eddies, is of order unity. At this point, the viscous forces become significant and dissipation starts to become important, and the rate at which the energy is passed down the energy cascade, at the smallest scales equals the rate of viscous dissipation of energy. These smallest scales are determined from the dimensional analyses and experimental investigations, and embodied in the definition of the Kolmogorov scales $[\mathbf{3} \boldsymbol{I}]$ :

- for the length scale: $\eta \sim l R e^{-3 / 4}$ (or $\left.\eta \sim\left(v^{3} / \varepsilon\right)^{1 / 4}\right)$,

- and the velocity scale: $v \sim(u R e)^{-1 / 4}\left(\right.$ or $\left.v \sim(v \varepsilon)^{1 / 4}\right)$,

$\eta, v$ and $\tau$ here are the length, velocity and time micro-scales, $v=\frac{\mu}{\rho}$ is the gas kinematic viscosity, and $\varepsilon$ is the dissipation rate of the turbulent energy. The Reynolds number, $R e=\frac{u l}{v}$, is here based on the macro-scale (the size of the largest eddies), and expressed in terms of $u$ - the macroscopic velocity and $l$ - the macroscopic length scale. We will investigate here to what extent the modelled geometries are meshed to these finest scales.

\subsubsection{Gas Spring Investigation and Results}

If one concentrates on the gas spring geometries that are investigated here, the largest length scale can at first instance be defined as the diameter of the piston (or possibly the height of the compressed domain - stroke plus the height of the 'dead' space), but since the compressed geometry changes dynamically, we will here propose to use the hydraulic diameter of the wetted area according to Hesselgreaves [32]. The characteristic length will then also have a time-dependent value:

$$
l=D_{h}(\theta)=\frac{4 V(\theta)}{A(\theta)}=\frac{D^{2} \pi(H+S-P(\theta))}{\frac{D^{2} \pi}{2}+D \pi(H+S-P(\theta))} .
$$

$H$ here is the 'dead' space height with piston in TDC position, $S$ is the piston stroke and $P$ is the instantaneous displacement of the piston from its initial position (BDC), in function of the crankshaft turn - crank angle $\theta$.

The characteristic velocity for the Reynolds number is also a transient value, in addition dependent on the coordinates of the region of interest. As there is no inlet nor outlet in the modelled geometries, the velocity of the gas in the axial direction changes along its height, from equal to the piston velocity at the piston crown, to zero at the cylinder top. In the same time, the velocity varies over the radius - near the wall the gas flow field is affected by the zero-velocity at the immediate wall boundary, and strongly differs from the 


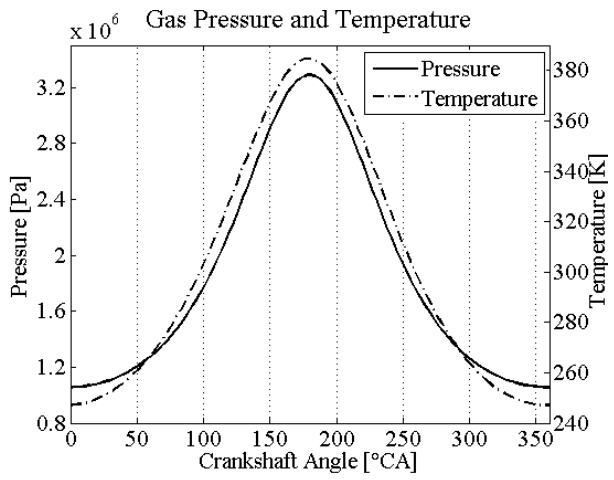

Figure 5.1: Typical pressure and temperature of the compressed gas during a compression cycle

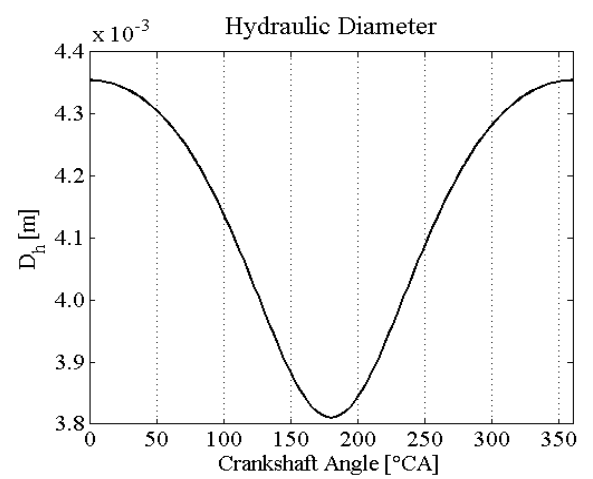

Figure 5.3: Hydraulic diameter in the compression cycle for the MIT geometry

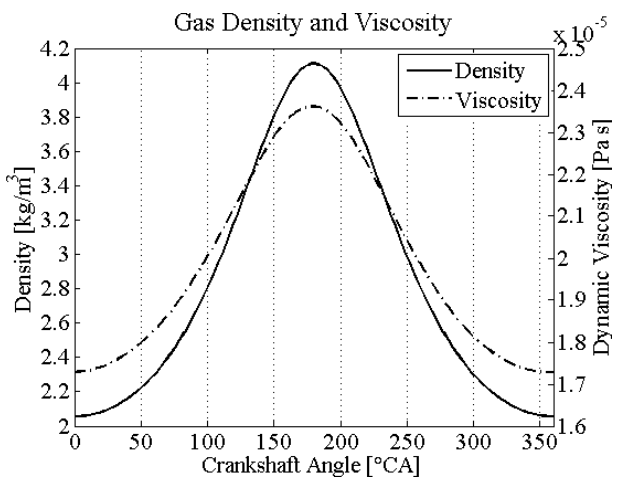

Figure 5.2: Density and viscosity of the compressed gas

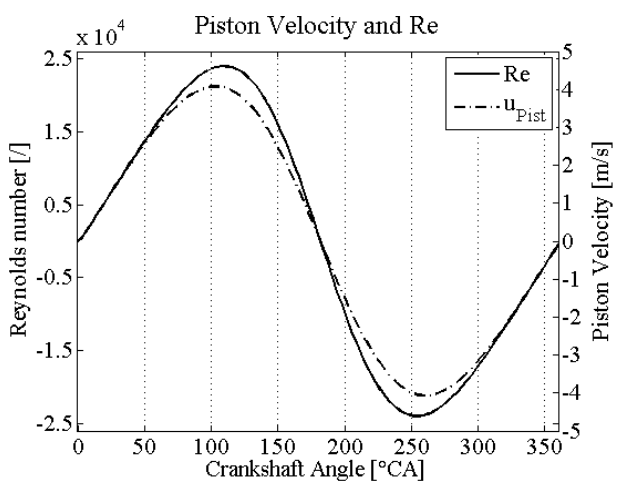

Figure 5.4: Piston velocity and Reynolds number for the $1000 \mathrm{RPM}$ run

unaffected core. Nonetheless, the critical value, leading to the highest Reynolds number and the highest chance of occurrence of turbulence, will be the highest velocity in the compression cycle, and in this view it is sensible to adopt the conditions at the piston surface as representative. The Reynolds number is then calculated as:

$$
R e=\frac{\rho u_{\text {Pist }} D_{h}}{\mu},
$$

and the turbulent length scale is:

$$
\eta=D_{h} R e^{-\frac{3}{4}}
$$


All quantities here: $R e, u_{\text {Pist }}, D_{h}$, and the kinematic viscosity $v$ are transient values, dependent on the part of the cycle, i.e. $R e, u_{\text {Pist }}, D_{h}, v=f(\theta) .3^{2}$

The simulation investigated here is the fast run (IOOO RPM) MIT model, presented in section 4.5 .2 , with the geometry meshed to $\sim 28000$ nodes. The initial and boundary conditions are already presented in Table 4.3 .

The results of these simulations are presented in Figure 5.I to Figure 5.4 as a function of the crank angle. The general thermodynamic properties of interest - gas pressure and temperature and gas density and dynamic viscosity are presented in Figure 5.I and Figure 5.2, respectively. In Figure 5.3 the change of the hydraulic diameter is displayed, and finally in Figure 5.4, the evolution of the piston velocity along the cycle and the respective Reynolds number are plotted.

Reading Figure 5.2, one can observe that the density has very high values, increasing from 2.I $\mathrm{kg} / \mathrm{m}^{3}$ to $4 . \mathrm{I} \mathrm{kg} / \mathrm{m}^{3}$ at TDC; this coming from the high charge pressure in the MIT experiment $-p_{\text {ini }}=\mathrm{I} 0.5$ bar. Dynamic viscosity is not affected by the gas pressure, and increases with the gas temperature 33 from $1.73 \mathrm{e}^{-5} \mathrm{~Pa}$ 's to $2.36 \mathrm{e}^{-5} \mathrm{~Pa}$ s. The hydraulic diameter ranges along the cycle between $43.5 \mathrm{~mm}$ and 38. $\mathrm{mm}$. Finally, with the highest velocity at the piston of $\sim 4 \mathrm{~m} / \mathrm{s}$ at $79{ }^{\circ} \mathrm{CA}$ before and after TDC, the Reynolds number calculated from Equation (5.2) peaks at

$$
R e_{\text {max }}=\frac{\rho^{\prime} u_{\text {Pist }, \text { max }} D_{h}^{\prime}}{\mu^{\prime}}=\frac{2.951\left[\mathrm{~kg} / \mathrm{m}^{3}\right] \cdot 4.058[\mathrm{~m} / \mathrm{s}] \cdot 0.041[\mathrm{~m}]}{2.0496 \cdot 10^{-5}[\mathrm{Pas}]}=23,952
$$

at $108{ }^{\circ} \mathrm{CA} 34$. Index ' marks the transient values. This calculated Reynolds number could strongly indicate a post-transitional, developed turbulent flow inside the gas spring [33].

Nevertheless, the next step is to define the characteristic length scale in the meshed geometries. The mesh for the mentioned MIT geometry with $\sim 28000$ nodes is once more presented in Figure 5.5. As described earlier, mesh is structured but not uniform, with symmetrically refined regions close to the surrounding walls and a coarser mesh in the centre of the domain. The radial dimension of the nodes varies from I $\mathrm{mm}$ at the centre

${ }^{32}$ For fast reference, this investigation could have been pursued with the cycle-mean parameters as approximation.

33 Gas dynamic viscosity change is defined in Sutherland's law $[\boldsymbol{8}],[59]: \mu=\mu_{0} \frac{T_{0}+C}{T+C}\left(\frac{T}{T_{0}}\right)^{\frac{3}{2}}$. Value of $\mathrm{C}$ in the above formula is for helium $79.4 \mathrm{~K}$ at $\mathrm{To}=273 \mathrm{~K}$.

34 The high initial charge here has an extremely strong influence on the Reynolds number, though the very high density. If compared to the same experiment, done on a gas spring charged to I bar (as in the experiments done at the University of Twente), and post-processed using the same criteria, Reynolds number peaks at only I460, thus almost twice an order of magnitude. 


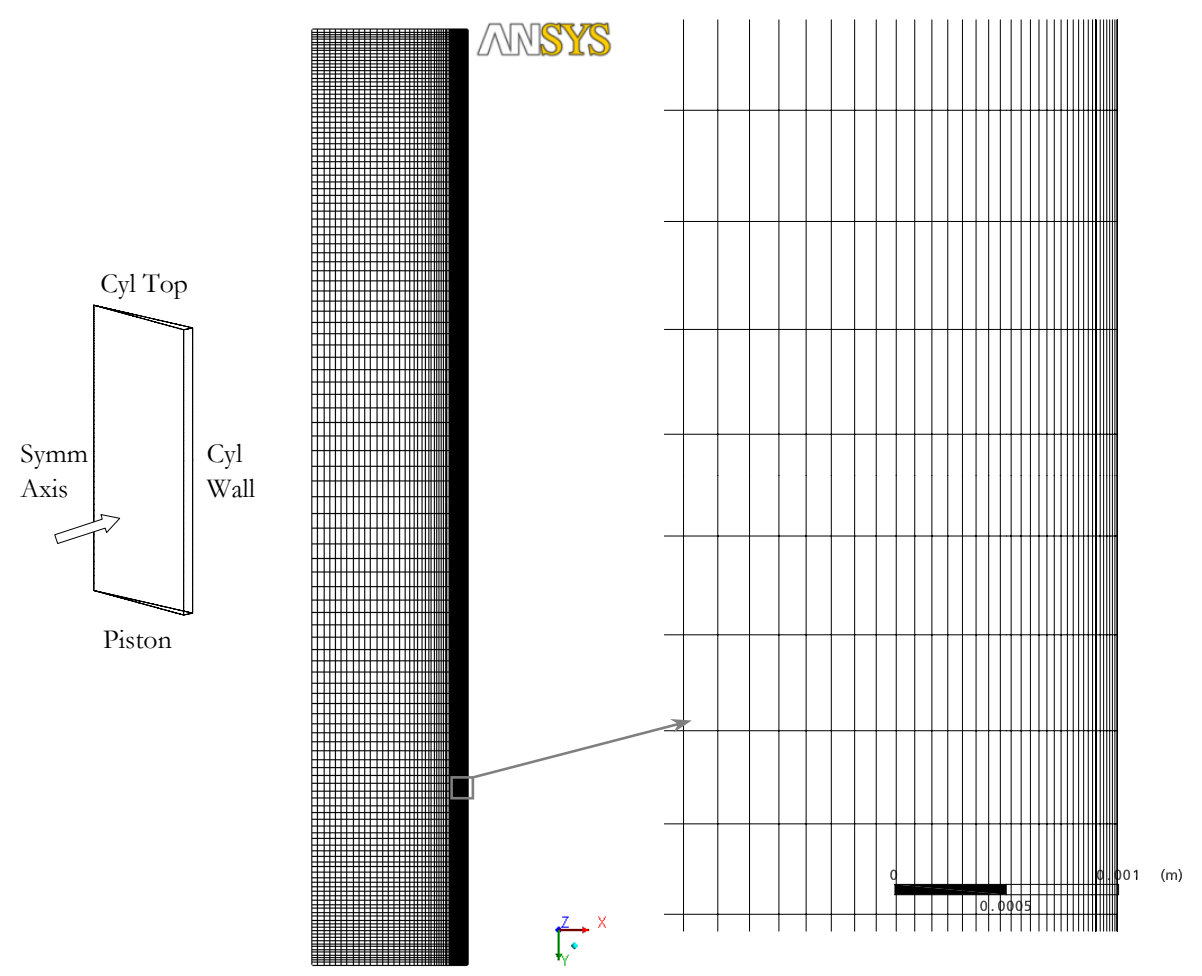

Figure 5.5: The compression-ratio 2 MIT gas spring model and the mesh refinement at the cylinder wall

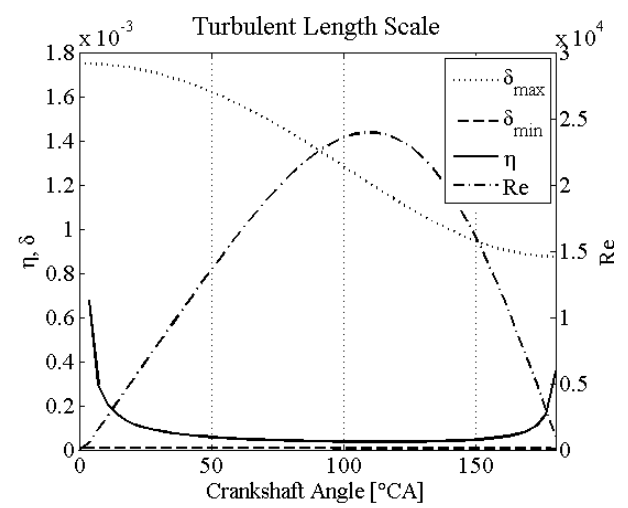

Figure 5.6: Kolmogorov turbulent length scale compared to the largest domain spacing $\left(\delta_{\text {max }}\right)$, and the smallest $\left(\delta_{\text {min }}\right)$ 
line down to O.OI $\mathrm{mm}$ at the cylinder wall 35 . In the axial direction, the proportion is somewhat different, and height of the cells ranges from $2.5 \mathrm{~mm}$ in the centre-axial plane to $\sim 0.4 \mathrm{~mm}$ at the horizontal boundaries. Nevertheless, this is before the compression, and the height of the cells will reduce proportionally to the compression ratio $(r=2$ in this case).

Thus the characteristic length scale is in this case not a constant and directly readable value, and the criterion for its selection is ambiguous. Once more assuming the worst-case parameter, the largest scale, of the central elements, is taken as representative. The central cells are I mm wide (cells width does not change with compression) and $2.5 \mathrm{~mm}$ high, and a mean arithmetic length between these two dimensions of $\delta=\mathrm{I} .75 \mathrm{~mm}$ is proposed and adopted. This value is accordingly also changing, proportional to the piston position.

Now the turbulent scale of the simulated numerical model can finally be evaluated. When the Kolmogorov scale is calculated according to Equation (5.3), the results presented in Figure 5.6 are obtained. The results are for relevance presented only for the compression stroke $\left(\mathrm{O}-\mathrm{I} 8 \mathrm{O}^{\circ} \mathrm{CA}\right)$, since the expansion process will be mirrored.

The turbulent Kolmogorov length scale based on the piston velocity ranges from infinitely large (for $u_{\text {Piston }}=0$ ) down to $0.022 \mathrm{~mm}$. In comparison to this, the maximum mesh spacing $\delta_{\max }$ is clearly much larger, and is approximately I.2 $\mathrm{mm}$ when $\eta$ is at its minimum $\left(\sim\right.$ IIO $\left.{ }^{\circ} \mathrm{CA}\right)$. This is almost two orders of magnitude higher. The smallest mesh size $\delta_{\text {min }}$ on the other hand is much smaller than the Kolmogorov length scale.

\subsubsection{Discussion}

Let's analyze these differences in comparison between the adopted largest mesh size $\delta_{\text {max }}$ and the Kolmogorov length scale $\eta$. The length scale model presented here is with a worst-case selection of parameters, aiming at the most unfavourable effects to the turbulent scale. Firstly, if we scale the characteristic velocity to the velocity at the central position (where the coarsest mesh is examined), and adopt its linear distribution in the axial direction, the gas velocity will be only half of that of the piston. This will directly result in Reynolds number equal to the half of the previous value $\left(\operatorname{Re}=\frac{1}{2 \operatorname{Re}_{\text {Pist, } \max }} \approx \mathrm{I} 2000\right)$, and further to the minimum $\eta$ of $0.036 \mathrm{~mm}$.

Further, the value of the representative mesh size is taken for the largest, central cells. Any occurrence of turbulence can indeed be expected in the 'free' core region, furthest from the influence of the stationary walls, but, as will also be discussed in subchapter 5.4, most of the visible irregularities in the gas movement is actually captured in the corner regions

35 Down to 0.00I mm for the UT models. 
of the domain, where the viscosity effects from the stationary bounding walls are the strongest. With the moving piston, the gas is pushed to shear with respect to the no-slip cylinder wall, and this is what will generate vortices and irregularities in the centre region as well. In this part of the domain, the size of the mesh cells is two orders of magnitude smaller, and plotted in Figure 5.6 as the dashed line. The thickness of these cells is less than the Kolmogorov-scale, and for the observed maximum Re moment, even 5 times smaller.

Finally, the applicability of the Kolmogorov principles as a mesh quality criterion is questionable for the application to the processes examined here. Kolmogorov hypotheses are based on the developed 'high-Reynolds-turbulent' pipe flows [3I], but a criterion for a 'sufficiently' high Reynolds number, in a geometry such as a wall-bounded compressed flow, with a small length/diameter ratio and no inlet and outlet, as the one examined here, is not clear. The transition range from laminar to turbulent flows of this type has not been extensively investigated and there are no such recommendations, to the author's knowledge. For the long and smooth pipe flows, the transitional regime between laminar and turbulent flow according to different authors ranges from $2300<\operatorname{Re}<4000$ [33] to $2300<\operatorname{Re}<\mathrm{Ie}^{4}[34]$. Over a flat plate, turbulence is ensured only for $\operatorname{Re}>\mathrm{Ie}^{5}$ [33]. It needs to be pointed out as well, that the mentioned high piston velocities and correlated Reynolds number are reached only for a very short time since the piston movement is sinusoidal.

Thus, it comes very difficult to say if the values presented here do indicate turbulent Reynolds numbers in the compression cycle and if the Kolmogorov criterion applies here for quantitative flow considerations. In parallel, and independent of these considerations, systematic refinement of modelled meshes has been pursued as described in section 4.I.2, and grid-independent solutions for the thermodynamic parameters of immediate interest to this research (gas pressure, temperature and wall heat flux), have been ensured. Meshing with parameters that would satisfy the Kolmogorov turbulent length scale in every region of the computed domain for even the fastest case presented here, would employ (apart from tedious meshing efforts) an extensive investment in computing costs to perform such simulations. ${ }^{36}$

\footnotetext{
${ }^{36}$ Some physicists today dispute the adequacy of Kolmogorov's dimensional reasoning [56]. The complete description of turbulence remains one of the unsolved problems in physics. According to an apocryphal story, Werner Heisenberg, a German theoretical physicist from mid-twentieth century, was asked what he would ask God, given the opportunity. His reply was: "When I meet God, I am going to ask him two questions: Why relativity? And why turbulence? I really believe he will have an answer for the first."
} 


\subsection{From Isothermal to Isentropic}

Work presented in this chapter describes the observations and theoretical discussion on the thermodynamic phenomena that emerged during the course of this research. Models on which these presumptions are validated are simulating the operation of the compression ratio 2 MIT setup, as examined on previous pages, and the two operational settings investigated in Chapter 4: typically very slow - 2 RPM, and reasonably fast operation - IOOO RPM. As discussed, due to the similarity of geometries, the discussion presented here can be extended to the UT and other similar geometries as well.

First discussed in this subchapter will be the phasing of the three examined thermodynamic system variables: gas pressure, gas temperature and the wall heat flux. Two mentioned operational cases can be understood as the reasonable boundaries of the expected functional settings, as a low-pressure/low-speed, and a high pressure/high speed gas spring, and thus convenient for examination of the occurring effects over this range. Correlation will be made with the behaviour in the near-wall regions and the boundary layer occurrence. Two cases will be further compared to the isothermal for the slow, and the isentropic cycle for the fast run, as theoretic analogies on the two limit cases, together with the discussion on the polytropic coefficients of compression and the compression work. Finally, compliance of the simulation results to the Clausius inequality law will be investigated.

\subsection{Phasing of Physical Properties}

Results for the simulated gas pressure and wall heat flux for the two mentioned MIT cases, validated with the experimental data were presented and quantitatively discussed in section 4.5.2. The third characteristic process property, the gas temperature, was not attended, and thus first a short discussion on the behaviour of the gas temperature during a compression process should take place here.

- Gas Temperature

Being that helium can be approximated as an ideal gas for the conditions investigated here [35], with the known pressure and mass of the gas, from the Clapeyron's equations the gas temperature is readily calculated. Nevertheless, by using a discretized numerical model such as the ones utilized here, one can determine and map the temperature and density (and any other property) of each computational cell of the domain. This can be interesting for determining the warm zones in the compressed volume, or estimate gas temperature at the exact position of a placed gas temperature sensor. Computation of the gas 
temperature, when compared to the thermodynamic analogy, also provides additional means of validating the energy balances in the modelled domain.

Due to the high thermal inertia of the solid walls surrounding the gas, as compared to that of helium, their temperature stays basically constant during a cycle. This forms strong temperature gradients in the gas, especially in the vicinity of the walls, and temperature varies in both radial and axial direction (this will be analyzed in more detail in Chapter 6). Simply volume-averaging the numerical results for the gas temperature over the compressed domain, and assuming this value as a domain-uniform equivalent, is a very close approximation, but still not entirely thermodynamically correct, and also slightly differing from the pressure calculated with the Clapeyron equation. Namely the density of the gas is not uniform either, and the mass factor of every finite volume should be taken into account as well when balancing the domain internal energy. With this reasoning, a density-weighted temperature is introduced. The numerically calculated gas temperature is stored for every time-step, and weighted with density over the domain volume (Favre averaging):

$$
\widetilde{T^{\imath}}=\frac{\int_{V} \rho_{j}^{i} T_{j}^{i} d V}{\int_{V} \rho_{j}^{i} d V}=\frac{\overline{\rho T}}{\bar{\rho}} .
$$

$i$ and $j$ here stand for the time and space coordinates, and ${ }^{\sim}$ for Favre and ${ }^{-}$for Reynolds averaging. The temperature calculated in this way matches the temperatures calculated via the ideal gas law to $0.0005 \%$, and thus on the level of numerical accuracy. The maximum errors that would be introduced by the volume averaging are higher but still moderate, and for the compression ratio 2 gas springs range from $0.7 \mathrm{I} \%$ for the slow run to $0.24 \%$ for the fast run.

Now we can go back to the phasing of the three gas properties. These are plotted in Figure 5.7, for the slow and fast run, normalized by their maximum values for convenient comparison, so that unity on the figures represents the maximum positive amplitude.

For the slow runs, the gas pressure peaks very close to the top-dead-centre (precedes it by approximately $2.5^{\circ} \mathrm{CA}$ ), and the gas temperature and wall heat flux are in phase. The heat flux here follows the heat transfer potential - temperature difference between the bulk gas and the wall temperature. The heat flux changes from positive to negative close to the $\mathrm{I} 8 \mathrm{O}$ ${ }^{\circ} \mathrm{CA}$ (zero flux occurs at approximately $\mathrm{I} 85^{\circ} \mathrm{CA}$ ), when the piston changes the direction of movement and the gas is basically quiescent. With expansion, the gas temperature becomes lower than the wall temperature, and with the increasing flow velocity this causes the occurrence of a negative heat flux. The negative and positive heat flux peak approximately $50{ }^{\circ} \mathrm{CA}$ after and before TDC, showing a direct correlation with the gas 

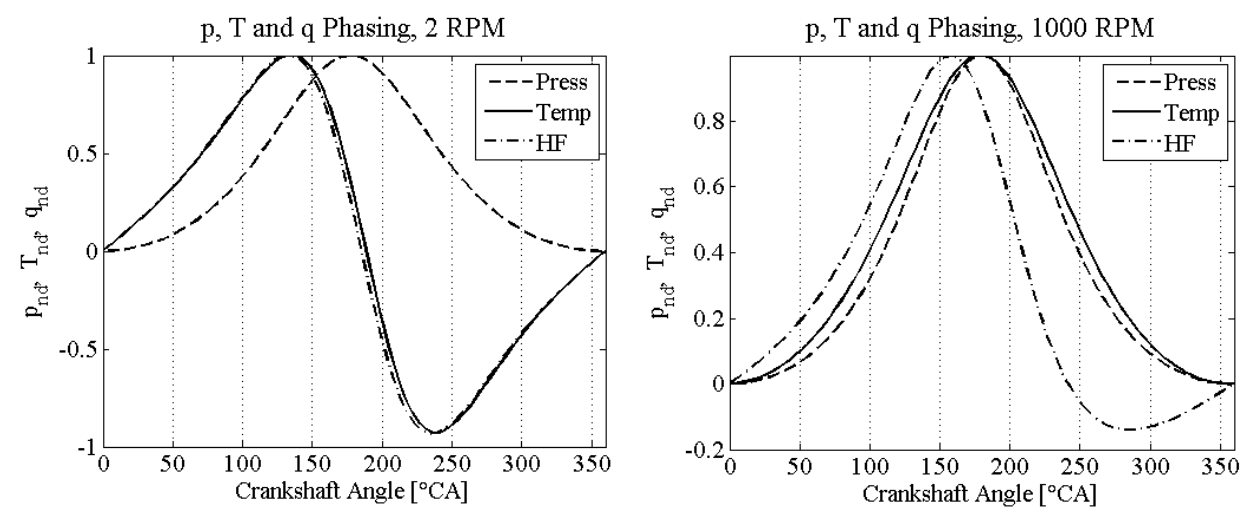

Figure 5.7: Phasing of the gas pressure, gas temperature and the wall heat flux for the slow run (left) and the fast run (right)

temperature, as obvious from the basically identical shape of the two normalized curves shown in the figure.

For the fast runs though, the temperature amplitude-wall heat transfer analogy fails (Figure 5.7 right). The gas temperature and gas pressure are now completely in phase, and peak very close to TDC (preceding it by $\sim 0.5{ }^{\circ} \mathrm{CA}$ ). The dynamics of the compression process here and the heat transfer cause the gas temperature not to be as obviously influenced by the wall heat loss as for the slower runs. In the 2 RPM gas spring, the compression process is very slow, and the compression work translated to 'excess' heat is being instantly transferred to the wall, in that way reducing the internal energy of the gas, and consequently its temperature. In the slow runs the temperature of the gas practically oscillates around the wall level37; in the vicinity of the TDC and BDC, when the piston comes to stand still and changes direction, gas velocities are very low and the gas looses heat by means of conduction to the regions close to the wall and approaches the wall temperature. In the fast run though, much more work is translated into the internal energy of the gas than taken away by the solid surroundings. The heat flux time curve for IOOO RPM is shifted 'to the right' as compared to the slow run and peaks at approximately $\mathrm{I} 55$ ${ }^{\circ} \mathrm{CA}$, thus approximately $65{ }^{\circ} \mathrm{CA}$ later than the maximum piston velocity moment, and 25 ${ }^{\circ} \mathrm{CA}$ earlier than the maximum temperature difference between the gas and the wall (for the slow run, the heat flux was directly proportional to the temperature potential). The zero heat flux also occurs later in the cycle, approximately at $240{ }^{\circ} \mathrm{CA}$. Other observed phenomena related to the phasing of described properties will be discussed in the following paragraphs.

\footnotetext{
${ }_{37}$ Gas temperature oscillation for the slow run is $\pm 5 \mathrm{~K}$, not visible here.
} 

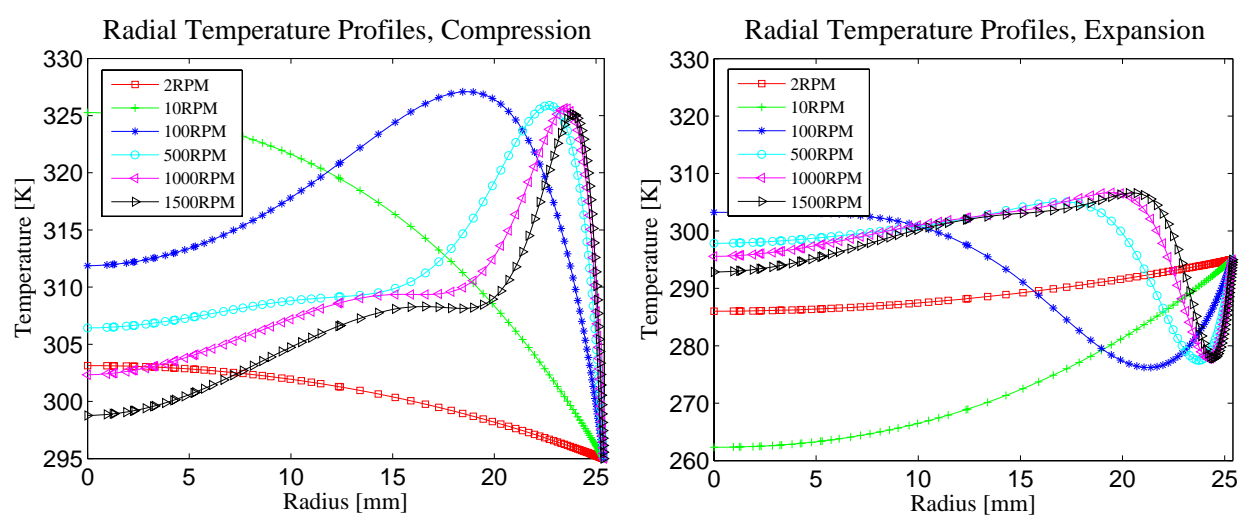

Figure 5.8: Temperature profiles in the radial direction, compression stroke (left) and expansion stroke (right)

\section{Influence of Temperature Gradients at the Wall}

For the sake of a better insight in the relations between the development of the temperature profiles in the gas and the heat transfer, a preview of the discussion on the thermal boundary layers from subchapter 6.4 is presented in Figure 5.8. Figure 5.8 presents the temperature profiles, for an extended range of operating frequencies, along the half-diameter of the gas spring and sampled from the computed gas temperatures along one radius in the domain (the method of obtaining these profiles and extended investigations are in detail presented in subchapter 6.4). Temperatures are sampled at the medium axial distance between the TDC and the cylinder top (38.I mm), and in the moment of the maximum piston velocities through the cycle $\left(79^{\circ} \mathrm{CA}\right.$ before and after TDC). As can be observed from the two figures, with the rising operating frequency, the gradients in the gas close to the wall become larger, the boundary regions become distinguished, and grow from a Gaussian-like distribution to very narrow close-to-wall regions.

This directly influences the phasing of the heat flux with the averaged gas temperature. In the slow runs, the temperature of the wall-adjacent gas layers is directly correlated to the core temperature and its amplitude is reduced and affected by the wall, but the temperature gradient along the radius is always uniformly positive or negative. This means that the gradient at the wall will also have the same sign as in the core, and the heat flux is then directly proportional to the bulk gas temperature.

During the high speed runs though, two distinguished regions in the compressed/expanded volume appear: the viscous boundary layer at the wall and the 
Radial Temperature Profile, $1000 \mathrm{RPM}, 240^{\circ} \mathrm{CA}$

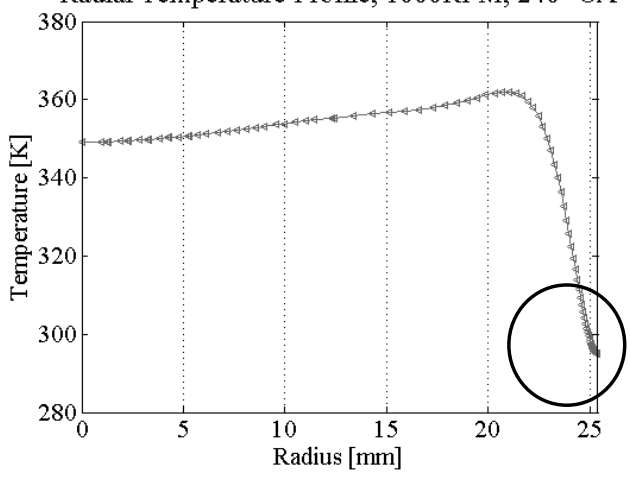

Radial Temperature Profile, $1000 \mathrm{RPM}, 240^{\circ} \mathrm{CA}$

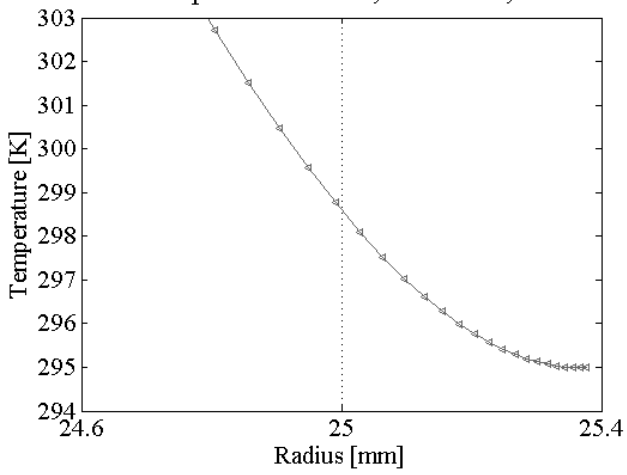

Figure 5.9: Gas temperature radial profile at $240{ }^{\circ} \mathrm{CA}$, along the radius and enlarged at the wall

inertial gas core. As the operating frequency increases, the boundary layers become narrower and the temperature gradient at the walls steeply rises, becoming completely 'detached' from the central region. Although according to some authors these regions can carry up to 30-40 \% of the compressed mass during expansion [36], still the main mass of helium is in the core and the averaged gas temperature will predominantly represent the temperature of the central region. In this situation the temperature of the gas at the wall boundaries thus cannot be directly correlated to the bulk gas temperature, and the steady-state gas-wall heat transfer analogies will fail.

In the same view, and stemming from the energy continuity at the gas-wall interface, the heat flux at the wall equals the temperature gradient in the gas at the immediate wall boundary, multiplied by the conductivity of the gas - Fourier's law:

$$
q_{x}=-k \cdot d T / d x
$$

If one looks closely to Figure 5.7, it can be observed that the heat flux in the fast gas spring will become negative (negative meaning the heat transfer occurs from the walls to the gas) at approximately $240{ }^{\circ} \mathrm{CA}$. In Figure 5.9 though, the temperature profile along the mid-'dead space' diameter of the gas spring at $240^{\circ} \mathrm{CA}$ is plotted, and it can be seen that the bulk gas temperature is significantly higher than the wall temperature (constant at 295 $\mathrm{K})$, while the temperature profile at the wall is horizontal - leading to a zero heat flux. This directly displays the inapplicability of the direct correlation assumption to general compression-process heat transfer. Even for a later expansion moment $\left(259{ }^{\circ} \mathrm{CA}\right)$, visualized in Figure 5.8 right, the bulk gas temperature for IOOO RPM is at, or higher than the wall temperature, while the gradient at the wall and the heat flux are already well negative. 


\subsubsection{Approaching Ideal Processes}

Since the here investigated compression processes are being viewed as two ends of the compression range and are named the typically slow and fast run, it was decided to investigate to which extent they actually do approach the two thermodynamic limits - the isothermal and isentropic compression process. Two pressure curves were thus calculated for comparison; one with the initial conditions as in the slow run and derived according to the isothermal compression law: $p V=$ const, and the second with initial conditions as for the fast run, simulating the isentropic change: $p V^{\kappa}=$ const. $\kappa$ is the isentropic compression exponent, and for helium $\kappa=1.67$ [23], [34]. These two curves are then plotted against the pressure simulation results already discussed here and in Chapter 4.

Results of this comparison are presented in Figure 5.IO. The slow run can be observed to be almost isothermal; its pressure curve almost perfectly follows the isothermal amplitude (overshoots it by $0.5 \%$ ), with only a small phase shift, preceding it by $2.5{ }^{\circ} \mathrm{CA}$. The fast run is very similar to the isentropic compression (adiabatic and reversible). The pressure amplitude is here underestimated by $3.2 \%$, and very closely matching its phase $\left(-0.5{ }^{\circ} \mathrm{CA}\right)$. This is also confirmed by plotting the polytropic exponents $n$ for the two processes in Figure 5.I I, recalculated from the polytropic change characteristic $p V^{n}=$ const for every time-step in the cycle. The analogy breaks down only at the start and end of the cycle, where the pressure $p$ and volume $V$ in expression

$$
n=\frac{\ln \frac{p_{0}}{p}}{\ln \frac{V}{V_{0}}}
$$

approach initial values $p_{0}$ and $V_{0}$ and result in a singularity. From another view, in these regions the piston comes to rest, no compression takes place and the only thermodynamic process occurring is the heat conduction through the gas and to the solids.

For the end of this section, it became interesting at this point to compare the compression work needed for these two typical settings, as well as the cyclic amount of energy transferred to the surroundings in the form of heat.

\section{Compression Work and Heat Loss}

Results for the pressure and wall heat flux have already been elaborated on in Chapter 4, and plotted in Figure 4.I 5 to Figure 4.I 8. Pressure-volume curves for these two cycles are presented in Figure 5.I2. The compression work for the fast run is of course several orders of magnitude higher, since the charge pressure is almost I 5 times that of the slow run (IO.49 bar as compared to 0.78 bar), and thus difficult to compare quantitatively. When integrated over one piston stroke (BDC to TDC), compression work for the slow 

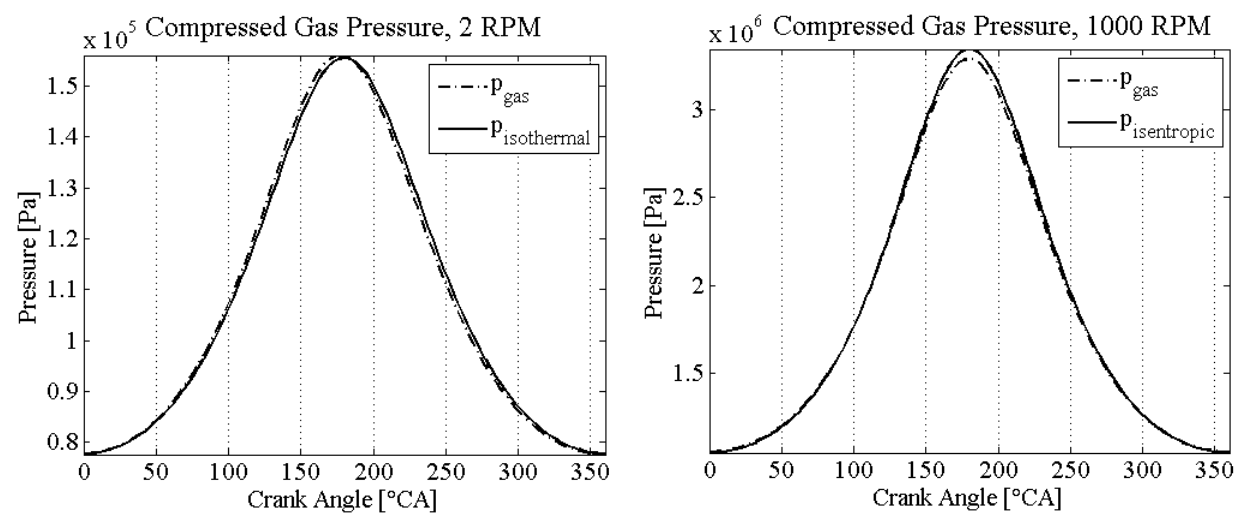

Figure 5.10: Approximation of the slow run by the isothermal compression (left), and of the fast run by the isentropic compression (right)

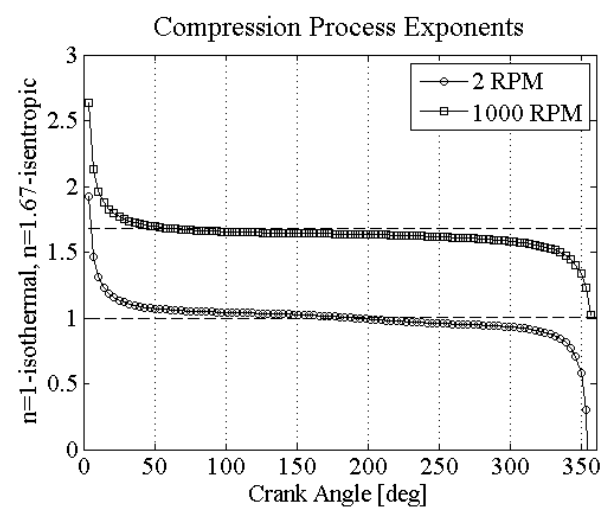

Figure 5.11: Calculated polytropic compression exponents for the slow and fast runs

run is $\sim \mathrm{I} 6.8 \mathrm{~J}$, whereas for the fast run it is $\sim 285.6 \mathrm{~J}$. What can be compared though, is the compression work for the two runs normalized with their adiabatic equivalents, and this is presented in Figure 5.I3. It can be once more observed that the fast gas spring operates almost adiabatically, and when integrated over the compression stroke, it requires over $99 \%$ of the adiabatic compression work. The compression work for the slow gas spring is lower, and requires approximately $80 \%$ of the adiabatic equivalent (compression exponent is much lower).

If the pressure is integrated over volume over the whole cycle though, one can obtain the work input, i.e. the heat loss over the steady-state cycle. This was done for the two cases, 


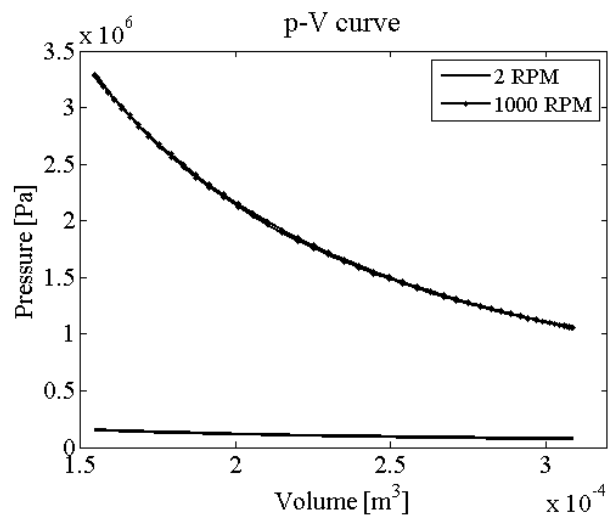

Figure 5.12: $p$-V curves for the slow and fast runs

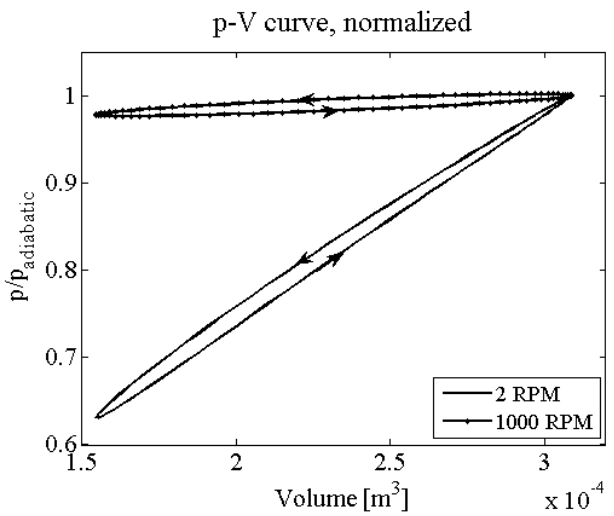

Figure 5.13: $p-V$ curves for the slow and fast run, normalized with the adiabatic compression work

\begin{tabular}{cccccc}
\hline $\begin{array}{c}\text { Piston } \\
\text { Work }\end{array}$ & $\begin{array}{c}\text { Charge } \\
\text { pressure } \\
{[\mathrm{Pa}] \cdot \mathrm{IO} 5}\end{array}$ & $\begin{array}{c}\text { Compression } \\
\text { Work [] }\end{array}$ & $\begin{array}{c}\text { Cyclic work } \\
\text { input J] }\end{array}$ & $\begin{array}{c}\text { Cyclic work } \\
\text { input/Compression } \\
\text { Work [\%] }\end{array}$ & $\begin{array}{c}\mathrm{p} / \mathrm{p}_{\text {adiabatic }} \\
{[/]}\end{array}$ \\
\hline \hline $\begin{array}{c}2 \\
\text { RPM }\end{array}$ & 0.7776 & $\mathrm{I} 6.8 \mathrm{II} 3$ & 0.3830 & 2.28 & 0.7950 \\
IOOO & I0.4859 & 285.6496 & 3.0007 & $\mathrm{I} .05$ & 0.9925 \\
RPM & & & & \\
\hline
\end{tabular}

Table 5.1: Piston work for the slow and fast compression cycles

integrating by the trapezoidal rule of integration [37], and these results are presented in Table 5.I. When normalized by the energy needed for the compression, the values basically represent the efficiency of the gas spring as a compression machine, in function of the operational parameters. It is observed that the work input is $\sim 2.2 \%$ of the compression work for the slow, and $\sim_{I} \%$ for the fast runs. This is also modelled analytically and presented in Chapter 6, for a larger range of operating conditions and two gas spring geometries.

Finally, following the first law of thermodynamics and the principle of conservation of energy, in a closed compression system with no occurring chemical reaction, such as the one investigated here, for a steady state operation the cyclic energy introduced to the system (by the compression work in this case), has to equal the energy to leave the system (heat loss to the surroundings). Thus the $p-V$ curves from Figure 5.I2 and the simulated heat flux from Figure 4.I6 and Figure 4.I8 were integrated over the full cycle and results were compared. The difference between these two amounts to the increase of the internal 


\begin{tabular}{ccc}
\hline $\begin{array}{c}\text { Cyclic Energy Balances } \\
\text { [J] }\end{array}$ & 2 RPM & IO00 RPM \\
\hline \hline Piston work & 0.3830 & 3.0007 \\
Heat loss & 0.3820 & 3.1043 \\
Int. energy increase & 0.0010 & -0.1036 \\
\hline
\end{tabular}

Table 5.2: Cyclic work input and the steady-state imbalance

energy of the system, i.e. the deviation of the system from the steady-state operation. These results are presented in Table 5.2, and it can be observed that these errors are small.

\subsection{Fluid Flow Patterns and Heat Transfer}

In connection to the considerations presented in the previous paragraphs, it was interesting to post-process the numerical simulations for visualization of the fluid flows and investigation of the development of fluid structures during one compression cycle. Since the fluid domain was carefully and sufficiently meshed for all the simulations presented so far, as explained in Chapter 4, these already computed cases could be directly and conveniently post-processed and used for this purpose. In this view, the fast run MIT case simulation (IOOO RPM) is taken in this chapter as generic, for which the visualization results and discussion are given.

Fluid flow development presented by streamlines, in 6 characteristic instants of a compression cycle, is displayed in Figure 5.I4. The figure shows half of a sampled interface-plane, with the lower horizontal line representing the piston, the upper line - the cylinder top, the left vertical boundary is the symmetry axis, and the right boundary - the cylinder wall. To remind, the piston, cylinder top and the cylinder wall are modelled as the solid boundaries with the no-slip condition imposed, and the piston is the only moving boundary. In the beginning of the simulation fluid is at a complete stand still (velocities of all nodes in the domain are equal to zero), with uniform properties - temperature, pressure and density, and the piston starts from the BDC position. To assist in comprehending the complete fluid-flow field, the shading on the sample plane represents the velocity field, with darker tones marking higher velocity regions and vice-versa, according to the accompanying legend. 

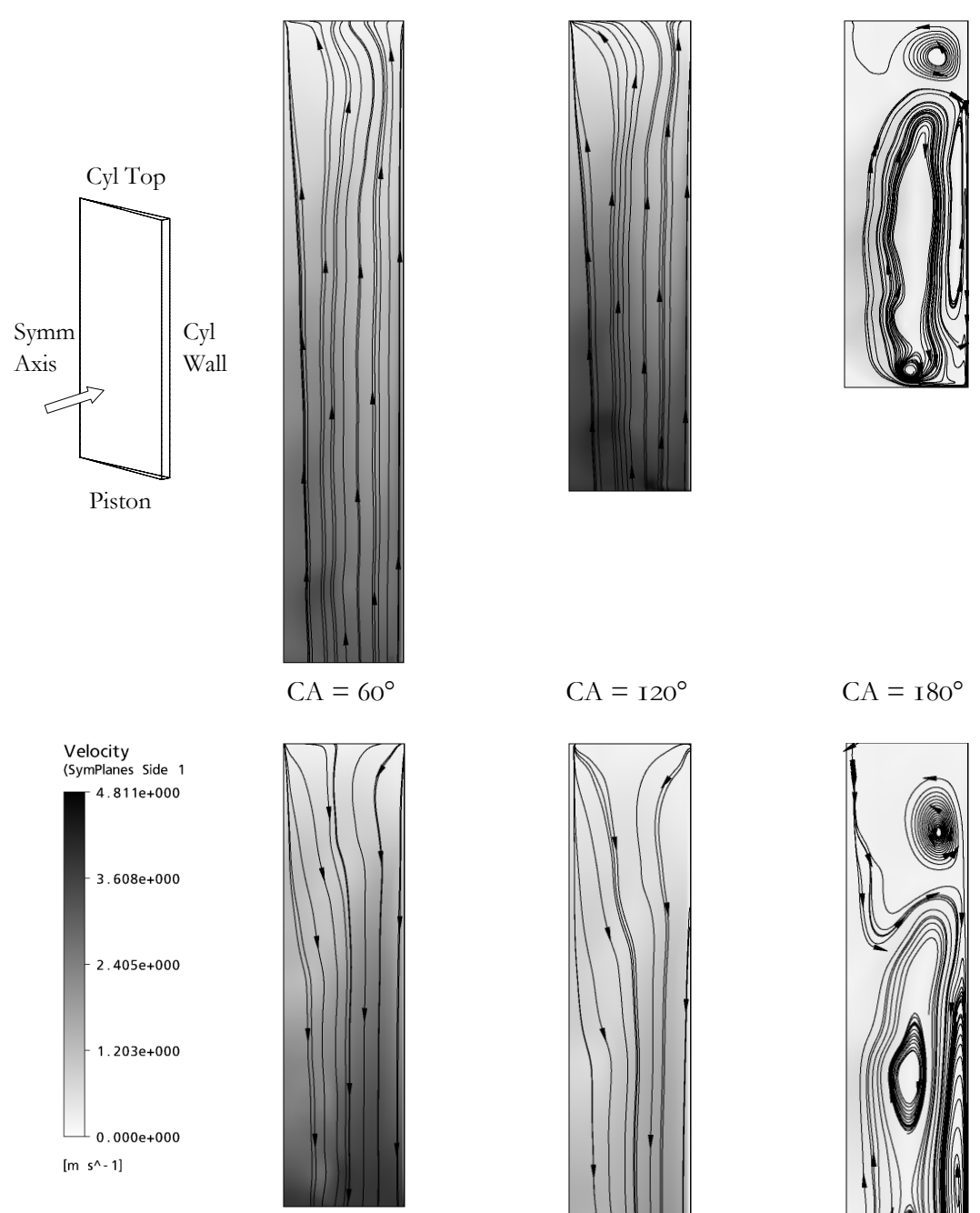

$\mathrm{CA}=\mathrm{I} 20^{\circ}$

$\mathrm{CA}=\mathrm{I} 80^{\circ}$

$$
\mathrm{CA}=60^{\circ}
$$

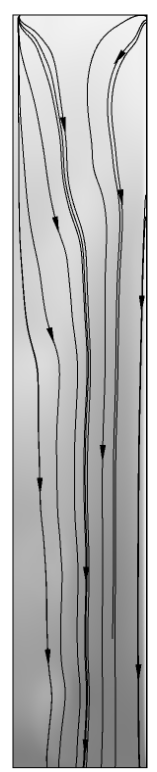

$$
\mathrm{CA}=240^{\circ}
$$$$
\mathrm{CA}=300^{\circ}
$$

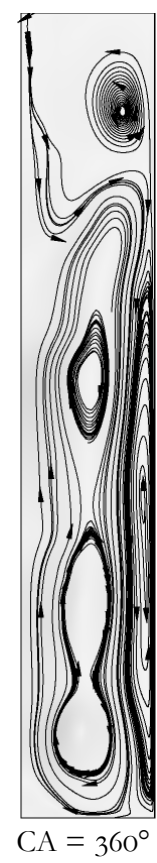

Figure 5.14: Velocity field and streamlines during one compression cycle 
In Figure 5.I 4 is displayed the $\mathrm{IO}^{\text {th }}$ cycle from the start of simulation, with flows fully developed and the gas spring in the steady-state operation. After passing the bottom-deadcentre, during the compression stroke, notable is the streamlines uniformity during the compression process. Fluid flow follows the naturally dominant axial displacement of the piston, with only some curving near the cylinder head region. Gas velocities are significantly higher close to the piston, with the fluid at the cylinder head appearing to be almost stagnant. In the moment of the change of direction of the piston movement, at the top-dead-centre, fluid viscosity and no-slip condition at the walls 'hold' the fluid particles close to the walls, while the rest of the domain is free to flow under the influence of the fluid's inertia and the driving motion of the piston. With the slowing down of the piston and then the change of the piston direction, local vortices are created that gradually lead to three main recirculation areas. One of them is on the cylinder wall boundary, one is in the 'dead' region of the cylinder and head corner, and the third is in the bulk gas area. It should be noticed, though, that the velocities of the fluid particles are very low in this part of the cycle, and these eddies appear to be very low energy structures. With the expansion, and with the piston moving in the other direction, the fluid flow again takes nearly structured vertical order, again up to the BDC moment, when vortices and recirculation areas re-appear.

Flow patterns mentioned above govern the heat transfer process during the compression/expansion cycle. During the upward and downward strokes, while the piston is moving with a significant velocity, flow inside the gas spring is very stratified and allows for high radial temperature gradients, in the conditions of very poor mixing of the fluid particles and with the heat transfer inside the gas and temperature equalization basically limited by the conductive properties of the gas. There is also no gas inflow or domain geometry to increase the mixing or stimulate the radial velocity component. Generation of vortices is caused only by viscosity-driven shearing effects in layers close to the wall; and the re-distribution of the gas temperature in the radial direction is very limited. With the low thermal conductivity of the gas, for the fast run this is a much slower process than the compression of the stratified vertical layers in the gas, imposed by the piston movement. This causes the specific temperature profiles shown typically in Figure 5.8. The fluid in the vicinity of the surrounding walls is strongly affected by the thermal mass of the solid and forms the thermal boundary layers, causing the density gradients as well (Figure 5.I5). These layers actively interact in the energy exchange with the core gas only in the moments of changing the piston motion direction, i.e. at TDC and BDC. The flow structure is then disturbed, and the gas from the core recirculates to the wall, only then forcing the actual convective heat transfer. In this way the instantaneous wall heat flux is once more shown not to be directly dependent on the temperature of the gas-spring core, but rather on the dynamics of the compression 


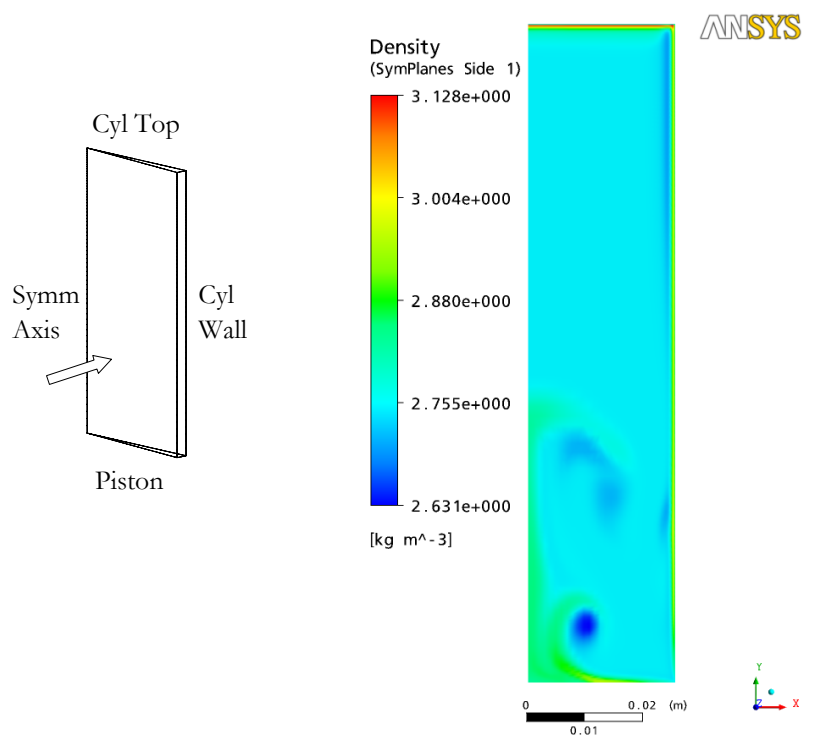

Figure 5.15: Typical density distribution, $80^{\circ} \mathrm{CA}$ after TDC

process, and end-of-stroke mixing of the fluid flows and the interaction between the core and the wall.

Figure 5.I6 displays the forming and disappearance of the vortices around the TDC in more detail. In Figure 5.I6, the interaction of the wall-affected layers and the shearing of adjacent streams is notable. The first vortices start to form in the corner of the cylinder wall and head, in the lowest velocity region. The fluid under inertia from the piston movement perturbs the near-to-wall region, creeps along it and causes a slow swirling motion of the gas and build-up of a vortex. A second vortex is formed at the cylinder wall boundary due to the no-velocity condition; with the large central vortex rotating in the clock-wise direction, while the sheering forces direct this new swirl in the counterclockwise direction and the core fluid is brought to the wall. With expansion, the vortex at the wall disperses first, and the central vortex is moved inwards. The latter swirl disappears; the vortices decay and the streamlines return to their vertically structured order.

\section{Turbulence}

Kornhauser [2] and Jak [27] in their work on the same test cases published considerations of turbulence occurring inside the same gas spring case, for the 'fast' run. In subchapter 5.2, the extent and the necessity of capturing the smallest turbulent scales were estimated 

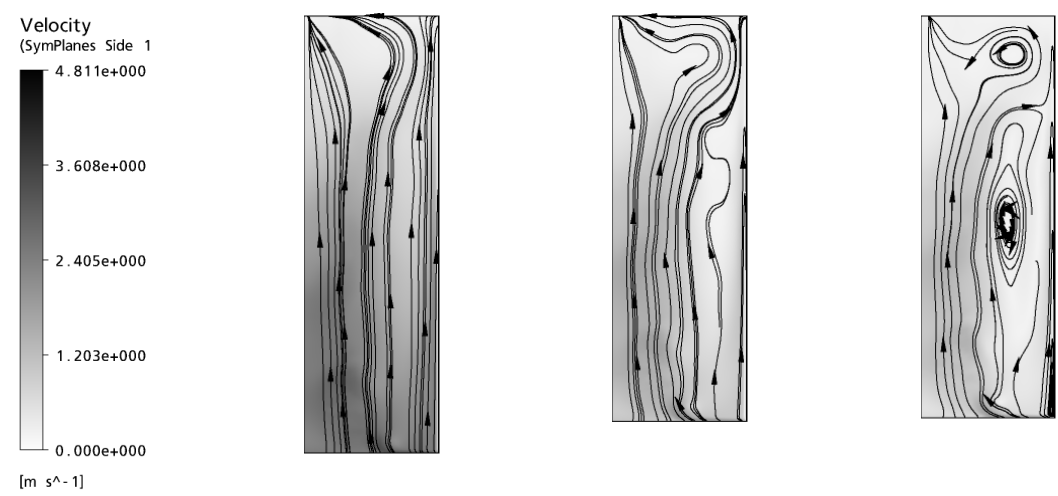

$\mathrm{CA}=\mathrm{I} 50^{\circ}$

$\mathrm{CA}=170^{\circ}$

$\mathrm{CA}=\mathrm{I} 75^{\circ}$
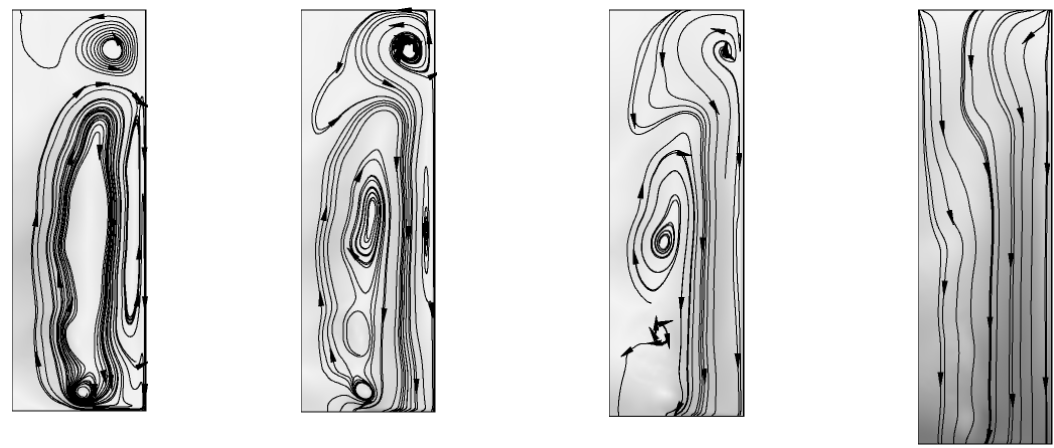

$\mathrm{CA}=\mathrm{I} 80^{\circ}$

$\mathrm{CA}=\mathrm{I} 85^{\circ}$

$\mathrm{CA}=\mathrm{I} 90^{\circ}$

$\mathrm{CA}=210^{\circ}$

Figure 5.16: Development of the flow patterns close to TDC

quantitatively, based on the peak computed Reynolds number and the Kolmogorov criterion. In addition to this, in the above qualitative discussion it is observed that even though the swirling regions are present next to the structured layers, this happens only in the low velocity parts of the cycle, thus with the Reynolds numbers very far below the established turbulence limits, or even approaching zero. In the moments of the maximum $\mathrm{R} e$ though, no swirling flow is visible in the compressed flow. Momentum imposed by piston, even in the high-speed part of the compression cycles, seems to be very quickly damped by the presence of the bounding walls and the slowing down and change of direction of the piston and the related fluid flow. In the absence of a fundamental research on the onset of turbulence and established turbulence criteria with respect to the here investigated types of flow, and based on the qualitative discussion presented above, it can thus be proposed that the observed 
flows can hardly be considered as turbulent (by the established fluid mechanics terminology).

Still, a conservative approach will very rightly propose further intensive investigations into the background of the numerical results obtained and presented here. In subchapter 5.2 it was shown that the best available computed Reynolds numbers could suggest at least a transitional regime, if not fully turbulent. In the author's previous work $[\boldsymbol{I} \boldsymbol{B}]$, numerical simulations using approximating turbulence models gave very different results for the heat transfer at the walls (and thus energy balances), which suggest very high dependency of this problem solutions and large errors imposed by using numerical models. The author thus eagerly proposes the investment of future efforts towards the investigation of alternative discretization techniques and understanding of the nature of their effects on the results of interest for these types of flows.

\subsection{Summary and Conclusions}

In this chapter, some additional questions, observations, discussions and conclusions that came along this research are presented. Results can be summarized as follows:

- Kolmogorov criterion is not directly applicable for the evaluation of computational domain mesh in numerical simulations pursued in this research. Non-uniformity of the mesh cell sizes and high gradients in the flow velocity hamper its use.

- For the typically slow runs (2 RPM), gas temperature and the wall heat flux are fully in phase and allow for a direct correlation between the gas-wall temperature difference and the instantaneous heat transfer. For the fast run though (IOOO RPM), this correlation fails and the heat flux can be viewed only to depend on the temperature gradient in the gas and existence of the boundary layers at the heat transfer boundaries.

- Slow run very closely approaches an equivalent isothermal compression process, whereas the fast run is well compared with the isentropic cycle. Polytropic compression exponents computed for the two runs confirm the isothermal $(n=1)$ and the isentropic $(n=\kappa)$ analogy for the slow and fast runs, respectfully.

- During the compression and expansion strokes the flow inside the gas spring is very stratified. The flow structure is only disturbed at the dead centre positions of the piston and very small fluid velocities, when some vorticing structures and swirling patterns can be observed. 
- This does not imply a turbulent nature of the flows inside the investigated gas springs, even though a maximum possible computed Reynolds number could suggest such a regime (with respect to the pipe-flow considerations). Suggestions are made to investigate whether the flows should be regarded as transitionally turbulent, fully understand the very high sensitivity of the obtained solutions to using turbulence models, and consider the employment of possible alternative discretization techniques to confirm the above presented conclusions on the flow characterization and development. 



\title{
CHAPTER 6 ANALYTICAL CORRELATIONS FOR GAS SPRINGS
}

\begin{abstract}
Many scientists in the past have attempted to define analytical correlations that will describe the thermodynamic behaviour of oscillatory thermal machines. These efforts, apart from the underlying theoretical stem, often have to feed on tedious experimental data in order to empirically support the approximating expressions for the phenomena of interest. Some correlations are specific and limited to a narrow range of investigated process conditions, while others can be applied to more general engineering systems. To either extent, they are used as practical tools in obtaining estimates for the investigated qualities; often as a first-guess calculator, to be followed by a detailed numerical or experimental research.
\end{abstract}

\section{I Introduction}

In previous chapters were presented experimental, numerical, and theoretical research work concerning the heat transfer in compression machinery and, more specific, in gas springs. A lot of effort was put in the construction of a novel experimental machine to directly measure the characteristic properties of interest, in the creation of numeric models in attempt to enable computer simulations that will validly represent the physical objects and occurring processes in scope, and finally, in continued analyses of the literature and knowledge available, in order to theoretically better understand the observed physical 
phenomena. This chapter concentrates on the fourth possible aspect of a scientific research; its objective is to analyze the analytical work on the described topics done to date of this publication, correlate them to presented results of numerical simulations and experiments, and propose new correlations or empirical corrections, where applicable. This work is presented in three main subjects, as summarized below:

\section{Cyclic Heat Loss, Pressure Magnitude and Pressure Wave Phase Shift}

Modelling of the heat loss in the gas spring is one of the main scopes of the research described in this thesis. Its occurrence in compression machines affects their performance in three ways $[\mathbf{3} 8]$ :

- compressed gas energy is dissipated (heat transfer hysteresis loss),

- pressure amplitude for a given piston displacement is reduced, and

- $\quad$ phase shift in pressure, relative to the piston displacement, occurs.

The objective of subchapter 6.2 is to correlate these effects to the numerical simulations for a range of operating frequencies and two different gas-spring geometries by an analytical model. An overview of the existing relations is presented. Variables are correlated to the parameters such as the compression ratio, the operating speed and the cycle mean pressure. Corrections in the parameterization that was proposed by previous authors are presented.

\section{Complex Nusselt Number and Instantaneous Heat Transfer}

The heat loss at the walls of a gas spring is governed by the instantaneous heat transfer potential - the difference in the temperatures of the solid wall and the gas in immediate contact with it. The today established and widely used analytical heat transfer models, based on Nusselt-Reynolds correlations, offer very attractive means for fairly accurate estimation of the convective heat transfer for developed flows. Nevertheless, the instantaneous convective heat transfer in oscillating machines is often out of phase with the temperature difference between the bulk gas and the counter surface - the cylinder wall. The proposed expressions incorporate a complex heat transfer coefficient as a function of the oscillation Péclet number, consisting of one part proportional to the gaswall temperature difference, and a second to the time rate of change of this difference. The analytical relations are validated by numerical simulations over a range of operating conditions and two gas spring geometries, and presented in subchapter 6.3.

\section{Thermal Boundary Layer Correlation}

The established boundary layer theory is applicable on the developed flows in certain typical geometrical arrangements (flows in annulus, flow over a flat plate). With the 
alternating compression and expansion such as in the geometries investigated here, the boundary layer structures form and disappear, and the related heat transfer processes have significantly different dynamics and relations than those established for most common developed flows. Subchapter 6.4 presents analytical expressions for the thermal boundary layer thickness developing in an oscillatory gas spring.

\subsection{Cyclic Heat Loss, Pressure Wave Magnitude and Pressure Wave Phase Shift}

\subsection{Background and Analytical Expressions}

\section{Literature Survey}

Heat loss has always been in the scope of interest of thermal research and development efforts in compression machinery. The earliest work in this direction was done already in the early I920's by Nusselt, Stokes, Reynolds, Prandtl and their contemporaries who created the concept of heat transfer coefficient and opened the door to analytical heat transfer analysis. Pfriem [39] in the I940's presented a one-dimensional theoretical analysis of engine heat transfer. In the late I960's, as a part of the internal combustion engines development, Annand [40] and Woschni [4I] proposed convective empirical correlations in the Nusselt-Reynolds form, based on the piston speed and estimated gas velocity. Breckenridge et al. [42], after first demonstrating that the wall temperature fluctuations would be negligible, approximated the temperature field in the gas, by superposing the uniform sinusoidal temperature fluctuation (of the gas) on a temperature field in a semiinfinite solid body, with sinusoidally varying wall temperature opposite in phase. The result was a constant surface temperature and oscillating core temperature. His result is widely quoted in the Stirling engine literature.

Later studies have been directed specifically at closed reciprocating engines. Faulkner and Smith [43], [44] conducted a series of experiments on heat transfer in a closed pistoncylinder apparatus, over a range of speeds, pressures and gases and presented different models to link their results to theory. Breckenridge et al.[42], Lee [45] and CookeYarborough and Ryden [46] modelled the gas-spring heat transfer based on onedimensional solid conduction. Lee and Cooke-Yarborough and Ryden solved the onedimensional energy equation for an ideal gas with sinusoidally oscillating pressure, neglecting convective terms and density variations. This resulted in equations equivalent to those of Breckenridge eta al. for a solid with a sinusoidal heat source/heat sink. Lee further made the assumption that a gas would have the same mixed-mean temperature as 
the solid and calculated the integral of $p d V$ over a cycle, whereas Cooke-Yarborough and Ryden calculated the integral of entropy generation over space and time, and thus hysteresis loss.

\section{Analytical Expressions}

Kornhauser [2], [47], [38] made extensive analyses of the extended analytical work done on these topics, and work presented in this and the following subchapter strongly relies on the results and proposals of his research. Additionally, he performed very extensive experiments including different gases, bore to stroke ratios and compression ratios, and even complex finned geometries, and created a strong database for the validation of the prediction models. Kornhauser based his work on the discoveries by Lee and CookeYarborough, non-dimensionalized their relations and extended their models to calculation of the pressure magnitude and phase shift relative to the cylinder volume curve. He also introduced a correction in one of the scaling factors used by Lee, as an improved fit for his experimental data. In his recommendations, Kornhauser limits the application of the correlations to light gases such as helium, and low compression ratios $(r \leq 2)$. This is not surprising, since the theory on which the expressions are developed, is based on small gas density variations, and fully sinusoidal pressure oscillation. Still, it was considered worth to examine whether the proposed expressions could be predictive for the two machines with a slightly higher compression ratio, examined in the research presented in this thesis.

Kornhauser's analytical expressions for prediction of the hysteresis loss, pressure wave magnitude and pressure wave phase shift are taken in their final form from his thesis, and are introduced as the basis for the work presented in this chapter. For a detailed derivation of expressions, the reader is suggested to Kornhauser's PhD thesis [2].

Analytic expressions for cyclic heat loss, compressed gas pressure magnitude and the pressure curve phase shift, as presented by Kornhauser, are:

- Hysteresis heat loss $L$ in $\rrbracket]$ :

$$
L=p_{0} V_{0} \frac{\pi}{2}\left(\frac{p_{a}}{p_{0}}\right)^{2} \frac{\gamma-1}{\gamma} \frac{1}{y}\left(\frac{\cosh y \sinh y-\sin y \cos y}{\cosh ^{2} y-\sin ^{2} y}\right)
$$

- and non-dimensionalized:

$$
L_{n d}=\frac{L}{p_{0} V_{0}\left(\frac{p_{a}}{p_{0}}\right)^{2} \frac{\gamma-1}{\gamma}}=\frac{\pi}{2} \frac{1}{y}\left(\frac{\cosh \mathrm{y} \sinh \mathrm{y}-\sin y \cos y}{\cosh ^{2} y-\sin ^{2} y}\right)
$$


- The non-dimensionalized pressure wave magnitude:

$$
p^{*}=\frac{p_{a}-p_{a, \text { isoth }}}{p_{a, a d}-p_{a, \text { isoth }}}=\frac{\gamma}{(\gamma-1)^{2} \sqrt{\left(\frac{1}{\gamma-1}+R\right)^{2}+I^{2}}}-\frac{1}{\gamma-1}
$$

- And the phase shift in [rad]:

$$
\phi=\phi_{p}-\phi_{p, i s o t h}=-\tan ^{-1}\left(\frac{I}{\frac{1}{\gamma-1}+R}\right)
$$

Parameter $y$ is initially defined by Lee:

$$
y_{\text {Lee }}=\sqrt{\frac{P e_{\omega}}{8}}
$$

and then corrected by Kornhauser:

$$
y=1.2 y_{\text {Lee }}^{0.86}=0.49 P e_{\omega}^{0.43}
$$

Variables in the presented equations are summarized below for convenience:

$p_{0}-$ cycle mean pressure,

$V_{0}$ - gas volume at mid-stroke,

$p_{a}$ - pressure wave magnitude, mean to peak (from the sinusoidal pressure wave assumption by Lee [45]:

$\left.p=p_{0}-p_{a} e^{i \omega t}\right)$,

$p_{a, i s o t h}, p_{a, a d}$ - pressure magnitudes for isothermal and adiabatic pressure wave for the same initial conditions and volume variation,

$\gamma-$ specific heat ratio,

$r$ - compression ratio,

$R=\frac{\sin y \cos y+\sinh y \cosh y}{2 y\left(\cosh ^{2} y-\sin ^{2} y\right)}$,
$I=\frac{\sin y \cos y-\sinh y \cosh y}{2 y\left(\cosh ^{2} y-\sin ^{2} y\right)}$,

$\frac{p_{a}}{p_{0}}=\frac{\frac{(r-1)}{(r+1)} \frac{\gamma}{\gamma-1}}{\sqrt{\left(\frac{1}{\gamma-1}+R\right)^{2}+I^{2}}}$

$P e_{\omega}=\frac{\omega D_{h}^{2}}{4 \alpha}-$ oscillation Peclét's number,

$\omega$ - angular frequency,

$D_{h}$ - hydraulic diameter at mid-stroke,

$\alpha$ - cycle mean thermal diffusivity. 
The analytical expressions above stated are in this research validated against the numerical simulations for two different geometries and a range of operating speeds and initial conditions.

\subsubsection{Numerical Database and Data Processing}

Two simulated geometries are modelled with respect to the MIT and UT gas springs (geometry specifics for these machines have already been elaborated on in Chapter 2 and Chapter 4). The investigated range of operating frequencies in this chapter is 2, IO, IOO, 500, IOOO, and I500 RPM for both geometries, and the operating gas is helium. Two different charge pressures are selected for a wider validation database: the initial pressures and temperatures for the MIT gas spring are $0.78 \cdot{ }^{1} O^{5} \mathrm{~Pa}$ and $295.3 \mathrm{~K}$, while for the UT setup they are $\mathrm{I} \cdot 5 \cdot \mathrm{IO}^{5} \mathrm{~Pa}$ and $295 \mathrm{~K}$. For every simulation, IOO readings of absolute gas pressure and crank-angle position are taken over one cycle. These numerical simulations serve as the validation tools for the analytical correlations presented below; the validity of the numerical models themselves is elaborated on in previous chapters. From these data and the calculated instantaneous domain volume, the numerically determined heat loss, pressure magnitude and pressure wave phase shift are calculated. The method of their derivation from numerical data is presented on the following pages.

- Cyclic heat loss:

A validation base for the cyclic hysteresis heat loss is calculated from the sampled numerically simulated instantaneous pressure and volume, by the trapezoidal rule integration of $p d V$ around the compression cycle:

$$
L_{\text {num }}=\oint p d V=\frac{1}{2} \sum_{i=1}^{n-1}\left(p_{i+1}+p_{i}\right)\left(V_{i+1}-V_{i}\right) \text {. }
$$

The parameters $i$ and $n$ here denote the time-step number of a sampling point in a numerically simulated cycle $(n=100)$.

This loss is then non-dimensionalized with the same factor as in the analytical model:

$$
L_{n d, n u m}=\frac{L_{n u m}}{p_{0} V_{0}\left(\frac{p_{a}}{p_{0}}\right)^{2} \frac{\gamma-1}{\gamma}}
$$


- Pressure wave magnitude

Kornhauser used the discrete Fourier transform of the first harmonic of the experimental pressure signal to evaluate the pressure wave magnitude and phase shift. This method is also presented in [2]. Nevertheless, with the computational means available at present it was preferred to create and use a Matlab code that processed the recorded pressure signals and directly calculated the two quantities of interest.

Since the analytical non-dimensional pressure magnitude $p^{*}$ relates the actual pressure magnitude to the magnitudes of the isothermal and adiabatic wave for the same volume change, they needed to be constructed for the numerical database too:

$$
\begin{gathered}
p_{i, \text { isoth }}=p_{\text {init }} \frac{V_{\text {init }}}{V_{i}}, \\
p_{i, a d}=p_{\text {init }}\left(\frac{V_{\text {init }}}{V_{i}}\right)^{\gamma},
\end{gathered}
$$

where $p_{\text {init }}$ and $V_{\text {init }}$ are the cycle initial pressure and volume and $V_{i}$ is the instantaneously captured volume.

The pressure magnitudes (mean to peak) were then created from the pressure wave curves of the simulated processes:

$$
p_{a}=\max (p)-\operatorname{mean}(p)
$$

The magnitudes for the isothermal and adiabatic equivalents were calculated analogously. The relative pressure magnitude was then:

$$
p^{*}=\frac{p_{a}-p_{a, i s o t h}}{p_{a, a d}-p_{a, i s o t h}}
$$

- Phase shift

Similarly to the pressure magnitude, the pressure phase is in place of the use Fourier coefficients defined directly from the pressure signals. Since the phase shift in Equation (6.4) is also expressed in the reference to the phase of the equivalent isothermal (or adiabatic) cycle and $\phi_{p, i s o t h}=\phi_{p, a d}=180^{\circ}$ relative to the volume wave, the phase of the sampled signal was defined by the angle of the recorded peak pressure and then deducted from $\phi_{p, i s o t h}\left(\phi_{p, a d}\right)$ :

$$
\phi_{\text {num }}=\phi_{p}-\phi_{p, i s o t h}
$$




\subsubsection{Results}

In order to create a validation database, the numerical simulations were performed prior to examining the performance of the above presented analytical correlations and comparison. Numerical models created for the MIT and UT experiments earlier described were used for this purpose, but with a set of operating conditions differing from ones presented in Chapter 4. This was done because the UT experimental setup was not ready for all measurements and valid operation until the last period of this research, and a provisional range of numerical validation data was in this way created far before the exact experiments were performed. Simulations were done with the compression ratio 2 numerical models; the operating gas is helium and no gas leakage during the compression cycle is modelled, i.e. the mass of the gas is fully conserved. The initial charge pressures were varied for the two different geometries, and both of them were simulated over a range of operating frequencies: 2, IO, IOO, 500, IOOO, and I50O RPM. All domain walls were specified as noslip wall boundaries with a constant temperature. The initial and boundary conditions are summarized in Table 6.I.

After the simulations were performed, the absolute gas pressure was domain-volumeaveraged and correlated to the instantaneous piston position. A Matlab code was then created and employed to post-process the data and compare the analytical and numerical results for the cyclic heat loss, pressure amplitude and pressure wave phase shift. Figure 6.I through Figure 6.3 show these results, with the numerically obtained result points presented with markers, and the analytical expressions plotted on top of them.

\begin{tabular}{cccc}
\hline $\begin{array}{c}\text { Initial and } \\
\text { boundary } \\
\text { conditions }\end{array}$ & $\mathrm{p}_{\text {ini }}[\mathrm{Pa}]$ & $\mathrm{T}_{\text {ini }}[\mathrm{K}]$ & $\mathrm{T}_{\text {walls }}[\mathrm{K}]$ \\
\hline \hline MIT setup & 0.78 & 295.3 & 295.3 \\
UT setup & $\mathrm{I} .5$ & 295 & 295 \\
\hline
\end{tabular}

Table 6.1: Initial and boundary conditions for the numerical simulations of the analytically modelled gas springs investigated in this chapter 
In the figures presented above, the quantities are plotted against the so-called oscillating ${ }^{38}$ Péclet number $P e_{\omega}[2]$, derived from the cyclic average Péclet number based on the hydraulic diameter:

$$
\begin{gathered}
P \dot{e}_{h}=\frac{\bar{\rho} c_{p} \omega S D_{h}}{\pi k}, \\
P \dot{e}_{\omega}=P \dot{e}_{h} \frac{\pi}{4} \frac{D_{h}}{S}=\frac{\bar{\rho} c_{p} \omega D_{h}^{2}}{4 k},
\end{gathered}
$$

with $\bar{\rho}$ as the cycle mean gas density, $c_{p}$ the specific heat at constant pressure, $S$ the piston stroke and $k$ the thermal conductivity of the compressed gas. The reasoning for the use of $P e_{\omega}$ over the Reynolds number is its convenience as presented by Kornhauser [2]: the Péclet number represents the ratio of the thermal energy conveyed to the fluid to the thermal energy conducted within the fluid, and in the context of a convoluted pulsating geometry (such as a gas spring investigated here), it can physically be understood as an inverted parameter of the dynamics of the heat transfer process with respect to the work input. When extended with the use of the hydraulic diameter in its formulation, it can be adjusted to compensate for different bore-to-stroke ratios. By normalizing the Péclet number with $\frac{D_{h}}{S}$, Kornhauser in this way obtained a process expression that is a function of operating frequency, hydraulic diameter, and gas properties, with no explicit gas velocity dependence.

The results of these simulations are presented with markers in the figures above: with squares for the MIT and circles for the UT simulations. They are named 'Geom' and 'Geom 2 ' since the intention here is only to numerically investigate the performance of the analytical models on two different geometries, i.e. two different bore to stroke ratios, and not the specific setups.

Now we can analyze the plotted figures. For low $P e_{\omega}$ (and low operating frequencies), Figure 6.I shows low cyclic heat losses. Operation in this region is nearly isothermal and the compression work is relatively small. The relative heat loss is also low at very high $P e_{\omega}$, where the operation approaches reversible adiabatic compression and the net work loss is small. At intermediate $P e_{\omega}$ though, the process is neither isothermal nor isentropic, and velocities and temperature differences are significant resulting in much higher losses. The peak loss occurs in the $P e_{\omega}$ range of approximately 2.5 to 25 , which stands for the IO-IOO RPM range for the presented 2 geometries and the presented set of operating conditions.

$3^{8}$ Formulation in Equation (6.I5) can be related to the oscillating flow Reynolds number $R e_{\omega}$ used in the oscillating flow studies, hence the name 'oscillating' Péclet number [2]. 

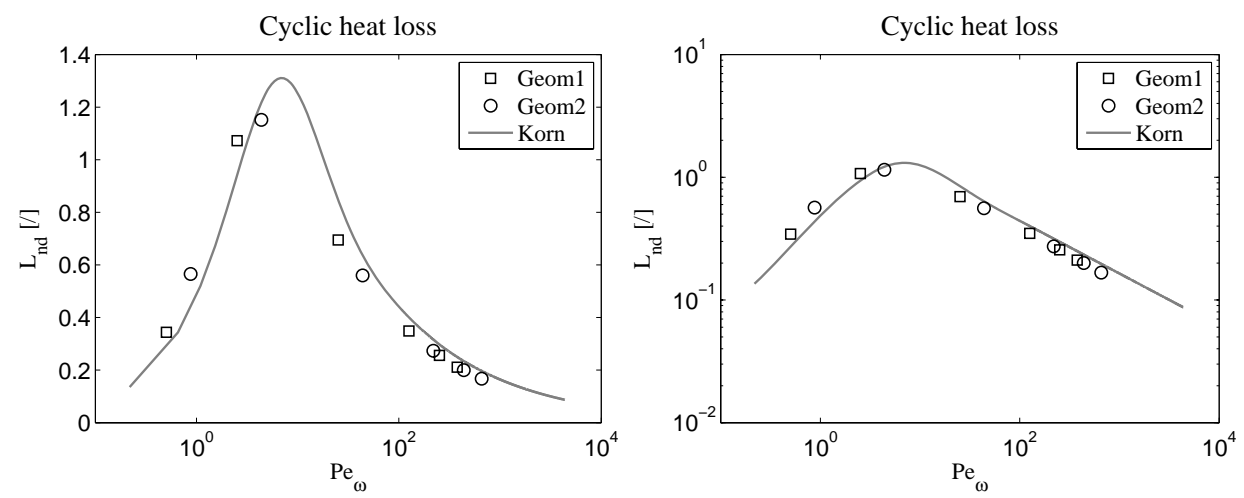

Figure 6.1: Hysteresis heat loss on the linear and logarithmic scale, and the analytic fit by Kornhauser

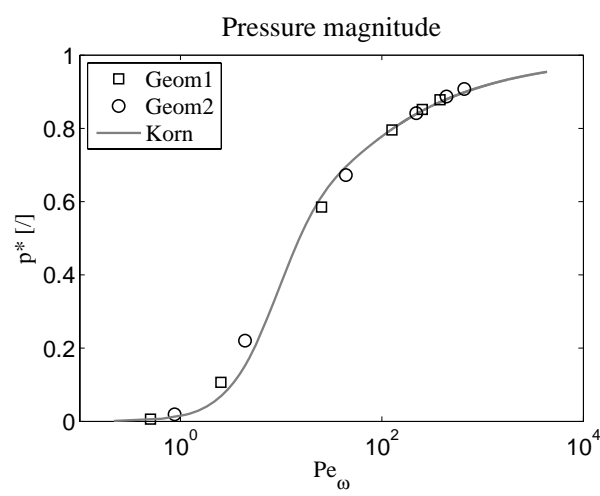

Figure 6.2: Pressure wave magnitude and the analytic fit by Kornhauser

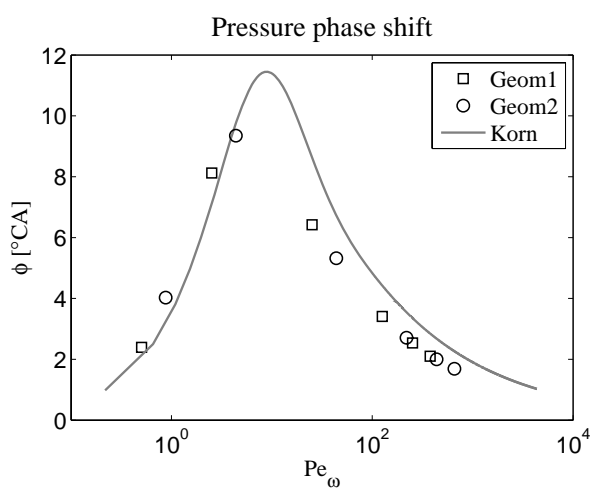

Figure 6.3: Pressure wave phase shift and the analytic fit by Kornhauser

If we further analyze the performance of the analytic expression, it can be observed that the non-dimensionalization correlates the data fairly well. Kornhauser also noticed that in the low $P e_{\omega}$ region, the non-dimensional loss increases proportionally to $P e_{\omega}$, while on the other side, at high $P e_{\omega}$ it decreases proportionally to $P e_{\omega}^{-1 / 2}$ (better visible on Figure 6.I right). The results presented here generally agree with those of Kornhauser, although the break-down of the correlation reported in his work is not observed here. Namely, Kornhauser argued the breakdown of the correlation by the Péclet number in the mid-Pé region (IO-IOOO for his experiments), reporting that for the same Péclet number, lowspeed high-pressure points would show greater loss than the high-speed low-pressure points. In the discussion presented here, two charge pressures and a range of operating frequencies (Table 6.I) were investigated and no such inconsistency was captured. Still, 


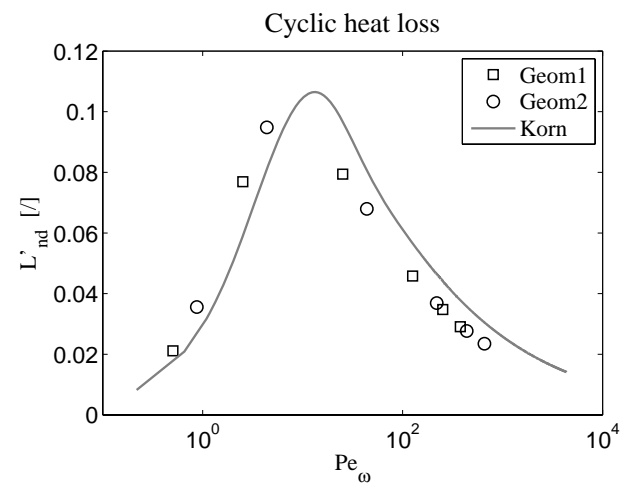

Figure 6.4: Pressure wave magnitude and the analytic fit by Kornhauser, normalized by the adiabatic compression work

possibly a wider range of charge pressures and $P e_{\omega}$ needs to be investigated for this, especially for the range concentrated in the highest loss region.

The non-dimensional pressure wave magnitude is displayed in Figure 6.2. As expected, $p^{*}$ increases from near zero at low Péclet numbers to near one at high $P e_{\omega}$, showing the full range of the possible thermodynamical behaviour in thermal machines (gas springs here) between the two theoretical extremes: from the near-isothermal system operating at low frequencies (2 RPM, Pé $\omega_{\omega}=0.5$ ), to near-isentropic at high operating frequencies (I50O RPM, $\left.P e_{\omega}=657\right)$. The analytical prediction performs here very satisfactorily.

Finally Figure 6.3 shows the pressure wave phase shift compared to the ideal compression process. The phase shift curve is very similar to that for the non-dimensional loss. Over the entire $P e_{\omega}$ range, the pressure leads adiabatic pressure wave; shift is rather small at low and high $P e_{\omega}$, and increases at intermediate $P e_{\omega}$. The maximum simulated phase shift is approximately $9{ }^{\circ} \mathrm{CA}$ at about $P e_{\omega}=9$.

In Equation (6.2), the normalization of the hysteresis loss is adopted as proposed in Kornhauser [38], and this loss is plotted in Figure 6.I. Nevertheless, this normalization factor does not provide sufficient insight in the quantitative magnitude of the loss, and for this reason the hysteresis loss was instead non-dimensionalized by the adiabatic compression work (work of adiabatic compression for the gas with same $p_{0}$ and $\left.V_{0}\right)^{39}$ :

39 This was also proposed in Kornhauser's earlier work [47] 


$$
L_{n d}^{\prime}=\frac{L}{W_{c o m p, a d}}=\frac{L}{p_{0} V_{0}\left(\frac{1+r}{2}\right)^{\gamma-1}\left(1-r^{1-\gamma}\right)} .
$$

The results are then re-plotted and the resulting graph is presented in Figure 6.4. It is now visible that the hysteresis loss in the simulated results ranges from $2 \%$ of the adiabatic compression work for $P e_{\omega}=0.5(2 \mathrm{RPM})$, to $\sim \mathrm{IO} \%$ for $P e_{\omega}=4.5(10 \mathrm{RPM})$.

\section{Correction Factors}

As explained, the full line plotted on the figures above represents the values calculated by the presented analytical expressions. It was observed that this fit could be improved for the here investigated range, and another empirical correction factor (next to Kornhauser's) is therefore introduced in the calculation of the parameter $y$ :

$$
\begin{gathered}
\text { corrfact }=1.15 \\
y_{\text {Lekic }}=\operatorname{corrfact} \cdot y=0.563 \mathrm{Pe}_{\omega}^{0.43} .
\end{gathered}
$$

Parameter $y$ in Equations (6.I) to (6.4) can now be replaced by $y_{\text {Lekic }}$, and once more compared to the numerically simulated values. Results of this correction are presented in Figure 6.5 to Figure 6.7. The corrected factor significantly improves the fit for the loss and phase shift, while the pressure magnitude still fails to provide a very good fit in the mid$P e_{\omega}$ region $\left(10<P e_{\omega}<100\right)$. As mentioned before, more efforts should be invested in this range to search for factors of influence and understand the discrepancy with the rest of the operating range.

A second empirical correction factor is proposed for the pressure wave phase shift data. It has to be noted that both corrections are obtained from a Matlab fitting procedure and do not hold a physical reasoning behind them; nevertheless, they correlate the discussed range of operating frequencies and charge pressures surprisingly well. The result for the proposed correction in Equation (6.4) is:

$$
\phi_{\text {Lekic }}=\frac{1}{\operatorname{corrfact}} \cdot \phi=-\frac{1}{\operatorname{corrfact}} \cdot \tan ^{-1}\left(\frac{I}{\frac{1}{\gamma-1}+R}\right) .
$$

Expression in Equation (6.19) is computed and presented in Figure 6.8. It follows that the pressure wave phase shift is now predicted much more accurately over the entire range of $P e_{\omega}$, including the predicted maximum values of the phase shift, as well as the decrease of it with increasing $P e_{\omega}$. 


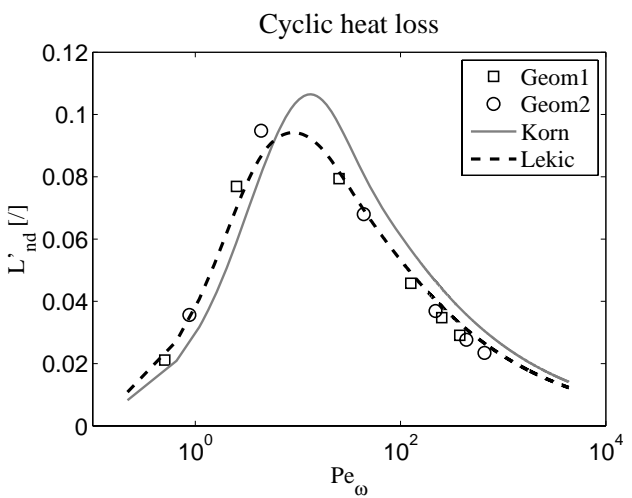

Figure 6.5: Hysteresis loss, improved fit by Lekic

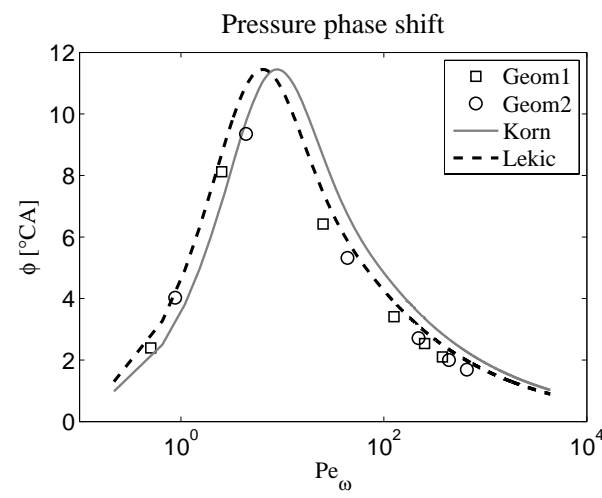

Figure 6.7: Pressure wave phase shift, improved fit by Lekic

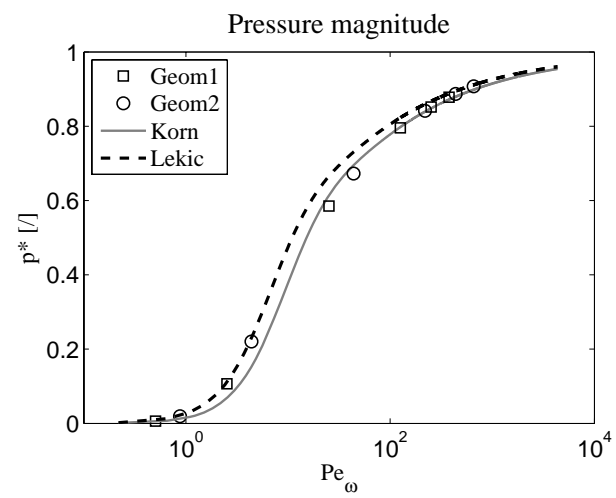

Figure 6.6: Pressure wave magnitude, improved fit by Lekic

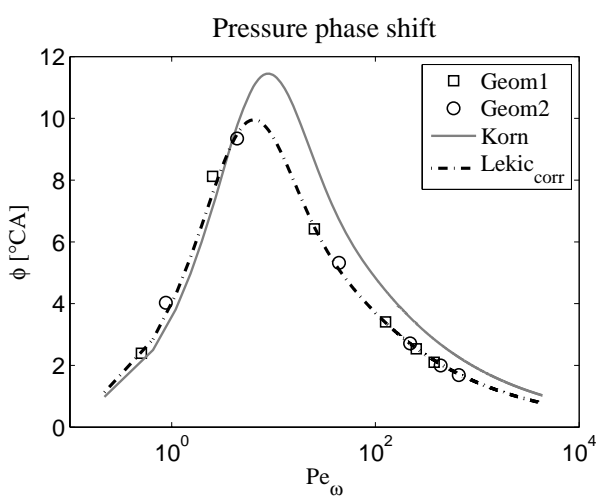

Figure 6.8: Pressure wave phase shift, with a second empirical correction

\subsubsection{Discussion}

The non-dimensional variables initially presented by Lee [45] and Cooke-Yarborough and Ryden [46], and reviewed by Kornhauser [2], [38], [47 correlate the investigated thermal phenomena - cyclic hysteresis heat loss, pressure wave magnitude and pressure wave phase shift, reasonably well over the investigated range of operating conditions for helium gas springs with compression ratio 2. The use of the oscillation Péclet number based on the hydraulic diameter as an independent variable (Equation (6.I5)) compensates for the variations in domain geometries, gas properties and operating frequency. The nondimensionalization of dependent variables compensates for different pressure amplitudes 
with operating conditions and allows for comparison with generic experimental and/or numerical data (Equations (6.2) to (6.4)).

Variables are correlated to the known volume compression ratio, operating speed and cycle mean pressure. The cyclic hysteresis heat loss, pressure wave magnitude and phase shift are shown to be fairly well predicted over the investigated range of operating speeds, initial pressures and geometries. The empirical correction factor for the expressions by Kornhauser is introduced and shows improvement in the analytical predictions (Equations (6.I7) and (6.I8)). The expression for the pressure wave phase shift can be further improved by the empirical correction shown in Equation (6.I9).

Considerations presented here can be further extended with closer investigation in the medium Péclet's number region, as this is the range of the highest hysteresis loss and pressure phase shift. This region is also reported in [2] to be a range where the correlations gave erroneous results, which is however not observed in the here presented work. In addition, expressions could be further evaluated on the higher compression ratios and for different operating gases.

\subsection{Complex Nusselt Number and Instantaneous Heat Transfer}

As indicated in multiple instances during this work, the instantaneous convective heat transfer, during compression and expansion in piston machines, is often out of phase with the bulk gas-wall temperature difference. Conventionally established heat transfer models are thus not capable of capturing this phenomenon. A heat transfer relation in these conditions must include a part other than the linear dependence on the gas-wall temperature difference. The expressions proposed in this chapter use a complex heat transfer coefficient, developed from simple heat conduction models. One part of the heat flux relation is proportional to the gas-wall temperature difference, and a second part to the time rate of change of this difference. The numerical simulations validate the analytic relation over a range of operating conditions and two gas spring geometries, using the same models as in the previous subchapter. The presented complex Nusselt number model shows to be reasonably successful in predicting the magnitude and phase of heat transfer in investigated gas springs. 


\subsection{Formulation}

Newton's law of convection directly relates the convective heat transfer, i.e. the wall heat flux $\dot{q}^{\prime \prime}$ to the heat transfer potential - the gas-wall temperature difference, through a heat transfer coefficient $h$. Nusselt number $N u$ is derived from it, as a non-dimensionalized engineering parameter proportional also to the gas conductivity $k$, and a flow-geometry characteristic length scale $L$ :

$$
\begin{gathered}
q^{\prime \prime}=h\left(T-T_{w}\right), \\
N u=\frac{h L}{k} .
\end{gathered}
$$

In the piston compression machines the in-cylinder heat transfer is often out of phase with the gas-wall temperature difference, and the correlation in Equation (6.20) fails. As proposed by Kornhauser [2], the Nusselt number and temperature can for this purpose be written as complex relations (real and imaginary part), and the hydraulic diameter $D_{h}$ can be taken as the characteristic length:

$$
\dot{q}_{c}^{\prime \prime}=\frac{k}{D_{h}} N u_{c}\left(T_{c}-T_{w}\right)=\frac{k}{D_{h}}\left(N u_{\mathcal{R}}+N u_{\mathfrak{J}}\right)\left[\mathcal{R}\left(T_{c}-T_{w}\right)+i \mathfrak{I}\left(T_{c}-T_{w}\right)\right] .
$$

$N u_{\mathcal{R}}=\mathcal{R}\left(N u_{c}\right)$ is the real part, and $N u_{\mathfrak{I}}=\mathfrak{J}\left(N u_{c}\right)$ is the imaginary part of the relation here. The temperature of the surrounding walls can be assumed to constant in time, as discussed earlier, so the wall temperature has no imaginary part. The real part of the expression for the wall heat transfer will then be:

$$
\mathcal{R}\left(\dot{q}_{c}^{\prime \prime}\right)=\frac{k}{D_{h}}\left\{N u_{\mathcal{R}}\left[\mathcal{R}\left(T_{c}\right)-T_{w}\right]-N u_{\mathfrak{J}} \mathfrak{I}\left(T_{c}\right)\right.
$$

For a sinusoidal temperature variation about a mean temperature $T_{0}$ with an amplitude $T_{a}$ and an angular frequency $\omega$, the complex temperature can be written as :

$$
T_{c}=\mathcal{R}\left(T_{c}\right)+i \mathfrak{\Im}\left(T_{c}\right)=T_{0}+T_{a} \cos \omega t+i T_{a} \sin \omega t
$$

and its time derivative

$$
\frac{d T_{c}}{d t}=-\omega T_{a} \sin \omega t+i \omega T_{a} \cos \omega t
$$

The imaginary part of the complex temperature is thus 


$$
\mathfrak{I}\left(T_{c}\right)=-\frac{1}{\omega} \mathcal{R}\left(\frac{d T_{c}}{d t}\right)=-\frac{1}{\omega} \frac{d \mathcal{R}\left(T_{c}\right)}{d t}
$$

and the heat flux

$$
\dot{q}_{c}^{\prime \prime} \equiv \Re\left(\dot{q}_{c}^{\prime \prime}\right)=\frac{k}{D_{h}}\left\{N u_{\mathcal{R}}\left[\mathcal{R}\left(T_{c}\right)-T_{w}\right]+\frac{N u_{\Im}}{\omega} \frac{d \mathcal{R}\left(T_{c}\right)}{d t}\right\}
$$

Finally, the heat flux in terms of the complex Nusselt number can now be written as:

$$
\dot{q}_{c}^{\prime \prime}=\frac{k}{D_{h}}\left[N u_{\mathcal{R}}\left(T-T_{w}\right)+\frac{N u_{\Im}}{\omega} \frac{d T}{d t}\right]
$$

where $T=\mathfrak{R}(T c)$.

\subsubsection{Analytical Expression}

The above presented idea is not recent, and numerous scientists in the past have attempted to correlate the experimentally obtained data with analytical expressions in the form of Equation (6.28). For the scope of this section, an overview of the most significant work is given here. Kornhauser [2] also gives an extensive review of the work done on this subject, and the reader is referred to his thesis for an additional survey.

\section{Background}

Pfriem [39] was one of the first to address this problem. He modelled the cylinder space with an one-dimensional energy equation in which he neglected convective heat transfer. In his model the space is divided in two regions: a conducting boundary layer at the wall and the uniform temperature core gas. Density variations are assumed small, geometry not deforming and the pressure oscillation was assumed to be sinusoidal. The method of complex temperature was used to solve the equation, resulting in a complex Nusselt number. Pfriem's complex Nusselt number is based on the difference between the wall temperature and temperature of the turbulent core gas.

$$
N u_{c, \text { Pfriem }}=D_{h} \Psi \frac{1-e^{-2 \delta \Psi}+L^{*} \Psi\left(1+e^{-2 \delta \Psi}\right)}{\left(1-e^{-2 \delta \Psi}\right)^{2}+L^{*} \Psi\left(1-e^{-2 \delta \Psi}\right)},
$$

with the variables: 


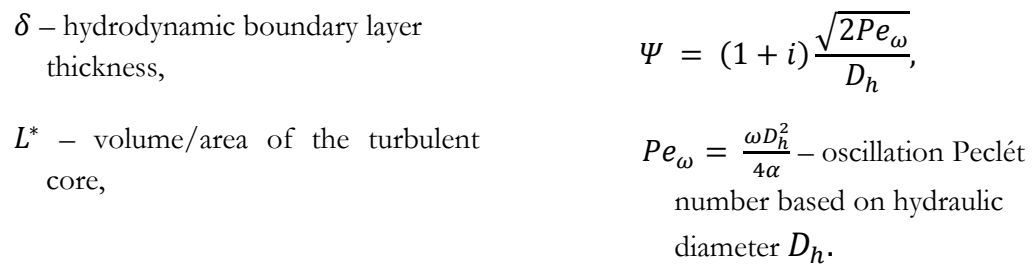

Annand and Pinfold $[48]$ measured the heat flux in a motored compression-ignition engine. Heat flux was here too found to be out of phase with temperature. They formulated a semi-empirical expression in the form:

$$
N u_{c, \text { AnnPinf }}=0.3 R e^{0.7}\left(1+0.27 \frac{D}{v \Delta T} \frac{d T}{d t}\right)
$$

Here $v$ stands for the instantaneous velocity, measured by a fine-wire element locally near the heat flux measuring point, and $R e$ is based on this $v . D$ is the cylinder diameter. This expression is essentially equivalent to the complex Nusselt number formulation presented in Equation (6.28). Put in a manipulated form of Equation (6.28), it reads:

$$
q_{\text {AnnPInf }}^{\prime \prime}=0.3 \frac{k}{D} R^{0.7}\left(T-T_{w}\right)+0.081 \frac{k}{v} R e^{0.7} \frac{d T}{d t} .
$$

Lee's [45] analysis was similar to Pfriem's. He solved the energy equation with the same simplifying assumptions, but based his model on the difference between the wall temperature and a gas mixed mean complex temperature ${ }^{40}$. Lee's complex Nusselt number was then, by using $P e_{\omega}$, put in the form:

$$
N u_{c, L e e}=\sqrt{2 P e_{\omega}} \frac{(1+i) \tanh z}{1-\frac{\tanh z}{Z}},
$$

with $z$ defined as

$$
z=(1+i) \cdot y_{\text {Lee }}=(1+i) \sqrt{\frac{P e_{\omega}}{8}} .
$$

${ }^{40}$ Gas mixed mean temperature is in [45] defined as: $T_{m m}=T_{0}+T_{0}\left(\frac{p_{a}}{p_{0}}\right) \frac{\gamma-1}{\gamma}\left(1-\frac{\tanh z}{z}\right) e^{i \omega t}$. $T_{0}$ here is the gas cycle mean temperature; other parameters are already mentioned. 


\section{Proposed Expression}

Kornhauser in his work [2], [49] reported Lee's expression to perform the best in correlating his very extensive experimental results over a wide range of operating conditions, bore-to-stroke ratios and working gases. Lee's expression - Equation (6.32) is also the one examined here and compared to the numerical data for validation. Real and imaginary parts $N u_{\Re}$ and $N u_{\mathfrak{J}}$ are then evaluated separately according to the complex expression in Equation (6.32): $N u_{\mathcal{R}}=\mathcal{R}\left(N u_{c}\right)$ and $N u_{\mathfrak{I}}=\mathfrak{I}\left(N u_{c}\right)$ and the procedure presented in the following section, for the later comparison with numerical results.

In addition, Kornhauser noticed the real and imaginary parts of complex Nusselt number to be approximately equal in magnitude and to have a power law relation with $P e_{\omega}$ in the high $P e_{\omega}$ region. For this reason, he performed a least-squares fit on the experimental data for $P e_{\omega}>100$, proposed $N u_{\Re}=N u_{\mathfrak{J}}$, and simplified the correlation to:

$$
N u_{\Re}=N u_{\mathfrak{J}}=0.56 P e_{\omega}^{0.69} .
$$

Nevertheless, this expression models the heat transfer only in the specified region of operating conditions (high Pé $e_{\omega}$ ), and thus, being not generic, will not be discussed here. In the following section the expression of Lee (Equation (6.32)) will be examined on the numerically produced data, and a correction for the parameter $z$, consistent with the empirical correction for $y$ presented in the subchapter 6.2 will also be evaluated. At the end, a fully empirical quadratic-law fits for the real and imaginary parts of the $N u_{c}$ are presented.

\subsubsection{Numerical Data Processing}

The validation base for Equation (6.32) is obtained from the results of numerical models already elaborated on in subchapter 6.2. The algorithm of processing the sampled data is presented below.

The analytical heat transfer expression to be validated is a direct function of $P e_{\omega}$ evaluated for the hydraulic diameter at mid-stroke and the cycle mean thermal diffusivity. The instantaneous $P e_{\omega}$, however, in reality does vary throughout the cycle as the density varies, and also will, much more intensively, the heat transfer coefficient. Nevertheless, for the purpose of validating the presented analytical correlation, since a cycle-mean $P e_{\omega}$ is correlated, a mean $N u_{c}$ was also created for every specific run as a best-fit for all the data in a cycle. For this purpose a least-squares technique [50] was used to determine a $N u_{c}$ which would best fit the temperature and heat transfer data for that run.

Discretization of Equation (6.28) yields [2]: 


$$
\dot{q_{l}^{\prime \prime}}=\frac{k}{D_{h}} N u_{\Re} \Delta T_{i}+\frac{k}{\omega D_{h}} N u_{\Im}\left(\frac{d(\Delta T)}{d t}\right)_{i},
$$

with

$$
\begin{gathered}
\Delta T_{i}=T_{i}-T_{w} \\
\left(\frac{d(\Delta T)}{d t}\right)_{i}=\frac{T_{i+1}-T_{i-1}}{2 \Delta t}=\Delta T^{\prime}{ }_{i}
\end{gathered}
$$

where $\dot{q}_{l}^{\prime \prime}$ is the instantaneous area-averaged wall heat flux, $T_{i}$ the instantaneous gas domain density-averaged temperature ${ }^{41}, \Delta t$ the time interval between the sampling instances $i$ and $i+1$, and $\Delta T^{\prime}{ }_{\mathrm{i}}$ is the gas-wall temperature difference time derivative, discretized by the central-differencing scheme. A least squares fit gave the real and imaginary part of $N u_{c}$ as:

$$
\begin{gathered}
N u_{\Re}=\left(\frac{D_{h}}{k}\right) \frac{\sum \dot{q}_{l}^{\prime \prime} \Delta T_{i} \sum \Delta{T^{\prime}}_{i}{ }^{2}-\sum \dot{q}_{l}{ }^{\prime \prime} \Delta T^{\prime}{ }_{i} \sum \Delta T_{i} \Delta T^{\prime}{ }_{i}}{\sum \Delta{T_{i}}^{2} \sum \Delta{T^{\prime}}_{i}{ }^{2}-\left(\sum \Delta T_{i} \Delta T^{\prime}{ }_{i}\right)^{2}}, \text { and } \\
N u_{\Im}=\left(\omega \frac{D_{h}}{k}\right) \frac{\sum \dot{q}_{l}^{\prime \prime} \Delta{T^{\prime}}_{i} \sum \Delta T_{i}{ }^{2}-\sum \dot{q}_{l}{ }_{i} \Delta T_{i} \sum \Delta T_{i} \Delta T_{i}{ }^{\prime}}{\sum \Delta{T_{i}}^{2} \sum \Delta{T^{\prime}}_{i}{ }^{2}-\left(\sum \Delta T_{i} \Delta T^{\prime}{ }_{i}\right)^{2}} .
\end{gathered}
$$

Here $\sum=\sum_{i=1}^{n}$ and $n$ is the number of sampled points per cycle.

This $N u_{c}$ is plotted against the mean $P e_{\omega}$ and presented on the following figures.

\subsubsection{Results}

A numerical database is created from the simulations already presented in Chapter 6.2. The complex Nusselt number was then calculated according to Equations (6.36) to ((6.39) from the post-processed numerical data.

Figure 6.9 shows the performance of the expression presented in Equation (6.32), as compared to the real and imaginary parts of the complex Nusselt number calculated from the numerical simulations. Analytical results for real and imaginary parts are presented by the full lines, and the numerical results for two geometries by the markers. Following the marking from the loss considerations from the previous subchapter, now squares and stars are used for the MIT geometry $\left(\mathrm{Geom}_{\mathrm{I}}\right)$, and circles and pentagrams for the UT setup $\left(G_{e o m}\right)$. It can be observed that the used complex Nusselt formulation correlates the data very well; data points are clustered in spite of two different geometries and different

${ }^{4}$ I Density-averaging routine (Favre averaging) is explained in detail in section 5.3.I. 


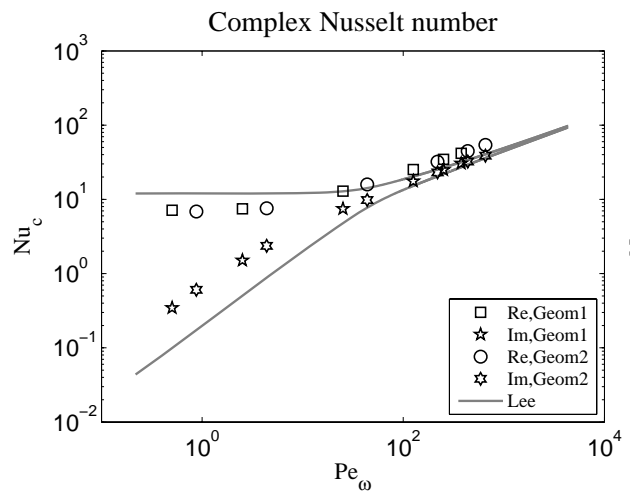

Figure 6.9: Analytical Nuc, vs. numerical data and fit by Lee

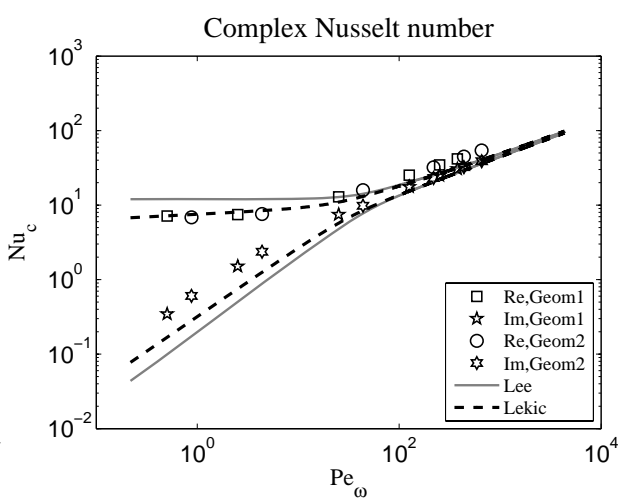

Figure 6.10: Improved analytical $N u_{0}$, Lee's expression corrected with $y_{\text {Lekic }}$

initial and operating conditions. The analytical fit by Lee qualitatevely follows the numerical data firly well - it has a similar trend, but fails to quantintatively closely predict the numerically obtained values for $N u_{\Re}$ and $N u_{\mathfrak{I}}$. It overpredicts the amplitude of $N u_{c}$ and underestimates its phase.

\section{Correction Factor}

In the hysteresis loss and pressure wave phase shift considerations, the introduction of a correction for parameter $y$ significantrly improved the analytical fit (Equation (6.I8)). In an effort to correct the defficiency of the model performance, it was decided to investigate if the expression of Lee can be somewhat improved by employing the same empirical correction here The parameter $z$ presented in Equation (6.33) was accordingly corrected by replacing $y_{\text {Lee }}$ with $y_{\text {Lekic }}$ discussed earlier in Equation (6.I8):

$$
z=(1+i) y_{\text {Lekic }}=(1+i) 0.563 P e_{\omega}^{0.43},
$$

and the performance of this model was examined. This is presented in Figure 6.IO.

Eventhough the fit is improved for the real part of the complex Nusselt number in the lower $P e_{\omega}$ region, for the higher $P e_{\omega}$ it is still underestimated, and this will cause an under-prediction of the heat flux amplitude. In the same time, the fit for the imaginary part of the expression is acceptable in the high- $P e_{\omega}$ region and with the correction slightly improved for low Peclét numbers, but still present in the entire range, and will still cause a phase shift in the heat transfer correlation. Effects of this are best visible in Figure 6.II. The heat flux is calculated with Equation (6.35) and the complex Nusselt number derived with this improvement, over the range of operating conditions and is here presented for 

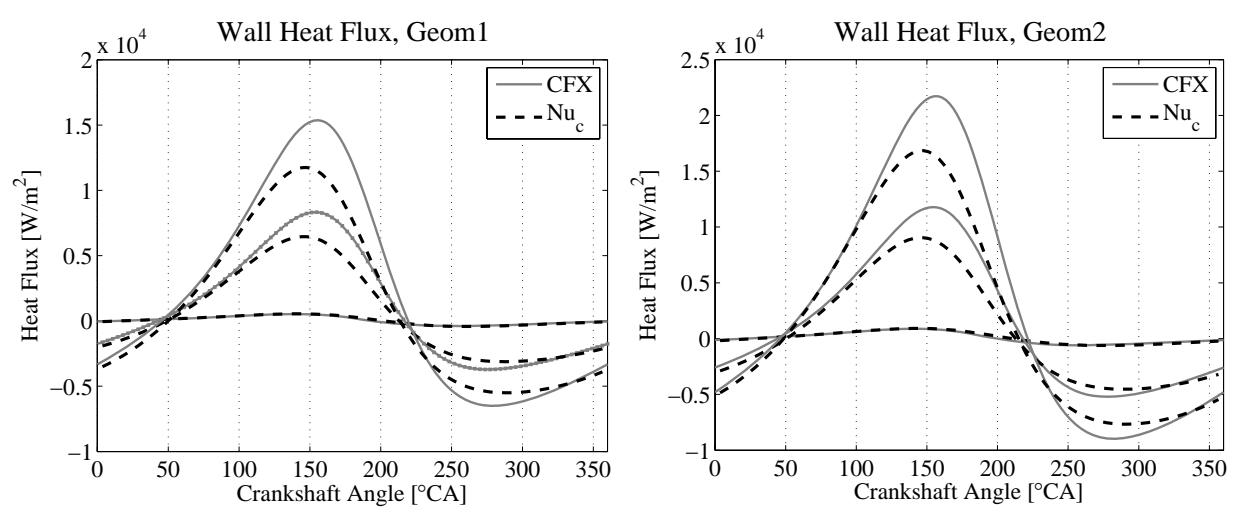

Figure 6.11: Heat flux determined analytically with by the expression of Kornhauser, plotted on the numerical data; MIT (left) and UT (right) geometry, 10, 500 and 1500 RPM

three operating frequencies - IO, 500 and I5OO RPM42. The shape of the heat flux curve is qualitatively reasonably well matched, but the values are still underpredicted by approximately $25 \%$ and advancing the numerical heat flux in phase by as much as IO ${ }^{\circ} \mathrm{CA}$. The change of direction of the heat flux though, positive being the heat flux in direction from the gas to the walls, is closely predicted. A conclusion that can be made here is that this discrepancy in the phasing and amplitude in parts of the cycle can be understood as inherent to this sort of approximation, having in mind that the presented analytical heat transfer correlation directly relates to the Péclet number averaged for the whole cycle and approximated by one, easy to evaluate cycle-specific value, while in reality, $P e_{\omega}$ is a dynamically changing quantity.

\section{Improved Empirical Fit}

Another observation lead towards an additional attempt on improving the complex Nusselt number fit. When a closer look at the shape of the real and imaginary curves in Figure 6.IO is taken, one can notice that they evolve by a power law change with respect to $P e_{\omega}$. Having in mind the lack of a very good correlation of the presented analytical relations, a curve fitting procedure was performed on the numerical data, and the result is a quadratic relation presented by Equation (6.4I):

$$
N u_{r, i}=c_{1} P e_{\omega}^{c_{2}}+c_{3}
$$

42 This corresponds to Péclet numbers 2.5, I26 and 377 for the MIT, and 4.4, 2 I9 and 657 for UT setup. Full range (2-I500 RPM) was computed for both geometries but only three frequencies are presented here for the readability of the figures. 
and the heat flux is further calculated as before, according to Equation (6.28). Two sets of coefficients, for the real and imaginary part, have accordingly been obtained. The coefficients are presented in Table 6.2.

Results of this empirical fit are presented in Figure 6.I2. The numerically obtained data for the complex Nusselt number shows now to be very well corrrelated over the whole range of $P e_{\omega}$, except for the only small dicrepancy in the phasing curve for $P e_{\omega}<1$. This refers to the operating frequency of 2 RPM, or $0.03 \mathrm{~Hz}$. For such slow runs the compression is nearly isothermal and the phase shift is very small, as also seen in the previous subchapter.

The heat flux computed using this fitted Nusselt number is displayed in Figure 6.I3. As can be seen from the figures, the wall heat flux is well correlated over the full investigated

\begin{tabular}{cccc}
\hline $\begin{array}{c}\mathrm{Nu}_{\mathrm{c}} \\
\text { coefficients }\end{array}$ & $\mathrm{c}_{\mathrm{I}}$ & $\mathrm{c}_{2}$ & $\mathrm{c}_{3}$ \\
\hline \hline $\mathrm{Nu}_{\mathrm{r}}$ & $\mathrm{I} .33$ & 0.56 & 5.36 \\
$\mathrm{Nu}_{\mathrm{i}}$ & 2.04 & 0.46 & $-\mathrm{I} .46$ \\
\hline
\end{tabular}

Table 6.2: Coefficients in the proposed empirical correlation for $N u_{c, e m p}$

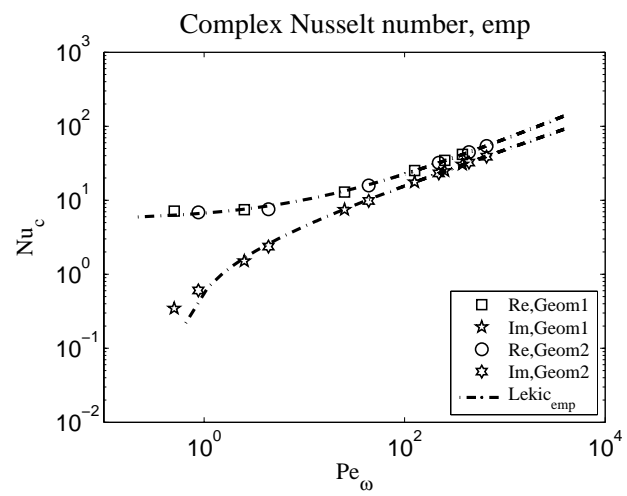

Figure 6.12: Empirically determined complex Nusselt number with expression (6.41) 

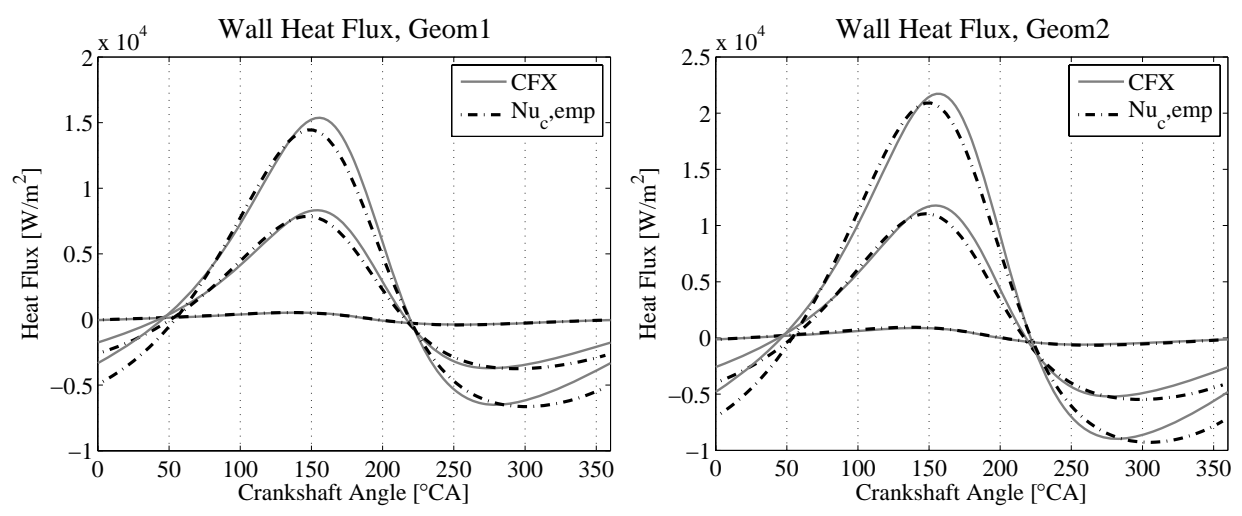

Figure 6.13: Heat flux derived with $N u_{c, \text { enp }}$; MIT (left) and UT (right) geometry, 10, 500 and 1500 $R P M$

range, and the fit compensates for the influence of all operating conditions (2-I50O RPM, 2 different geometries and different initial conditions). The heat flux magnitude is very closely matched, while the phase is still predicted to advance by approximately IO ${ }^{\circ} \mathrm{CA}$ for the fastest run. A larger inaccuracy is noticed at the expansion end; the predicted heat flux is again matching the amplitude well, but is late in phasing by as much as $25{ }^{\circ} \mathrm{CA}$.

\subsubsection{Conclusions}

The heat transfer is often out of phase with the bulk gas-wall temperature difference in reciprocating gas springs. Steady state convective heat transfer correlations are inadequate to describe this phenomenon. Researchers in the past (Pfriem, Annand and Pinfold, Lee, Kornhauser) have expressed the rate of heat transfer in terms of a complex Nusselt number, as the sum of one part proportional to the gas-wall temperature difference and another proportional to the rate of change of temperature (Equation (6.28)). Lee [45] and Kornhauser [2] relate $N u_{c}$ to a mean oscillation Péclet number based on the hydraulic diameter. The Péclet number effectively correlates the real and imaginary parts of $N u_{c}$, for the numerically obtained results taking into account the influence of different geometries, gas properties and operating conditions.

The analytical expression derived by Lee and corrected by Kornhauser shows to be reasonably successful, but still not accurate enough to be used as the sole method for modelling the helium gas spring heat transfer. A correction in parameterization presented in Equation (6.40) and in accordance to the considerations presented in subchapter 6.2 somewhat reduces the analytical modelling errors, but still allows for the under-prediction of the heat flux amplitude of $\sim 25 \%$, and a phase shift of $\sim$ IO ${ }^{\circ} \mathrm{CA}$. 
In another approach, the real and imaginary parts of the complex Nusselt number show a power-law relation to the oscillation Péclet number and can be expressed in the form $N u_{r, i}=c_{1} P e_{\omega}^{c_{2}}+c_{3}$, with empirical coefficients $c_{1}$ to $c_{3}$. The heat flux calculated in this way predicts the experimental data over a wide range of operating conditions well in amplitude, but still introducing a phase error of the same magnitude as the other presented analytical models. The presented analytical and empirical expressions still can be used as a first-guess tool for heat flux prediction in gas springs, provided that cycle-average gas properties employed in these models can be estimated with reasonable certainty.

\subsection{Thermal Boundary Layers in Gas Springs}

\subsection{Introduction}

Heat flux at the cylinder walls of an operating gas spring is the measure of transfer of energy from the operating gas to the solid walls in contact with it, and vice-versa. It is driven by the heat transfer potential at this interface - the difference between the temperature of the solid walls and the temperature of the gas in contact with it. With compression and expansion, the moving gas in immediate contact with the stationary walls undergoes processes of significantly different nature than the rest of the volume. Capturing and understanding this near-wall physical behaviour of the compressed gas is a step towards a better understanding of the occurring internal heat transfer processes, and a significant topic that was attended on several occasions in this thesis. This subchapter reports on the final efforts made to analytically describe the formation of the boundary layers and to this related observations, in order to supplement and conclude the extensive work discussed in the hitherto presented paragraphs and pages.

Behaviour of the gas in the close-to-the-wall regions in every instant of a compression cycle is determined by several important physical properties and phenomena: in the first place the gas density and viscosity, and then also the dynamics of the compression/expansion process, heat conduction in the gas, shearing effects and friction imposed by the roughness of the walls, and heat convection mechanisms from the gas to the solid. The rate of the heat transfer and its phasing with respect to the 'measurable' gas properties - the gas pressure and the bulk-gas temperature, are thus determined by the characteristics of the specific regions between the wall and the core gas - the gas boundary layers. In addition, the described regions at the bounding walls can carry a significant part of the volume-enclosed gas mass (30-40 \%, [5I], Figure 6.I4). The boundary layers are usually conceptually correlated to the presence of turbulence, but, although swirling motion can 


\section{Density}

(SymPlanes Side 1)

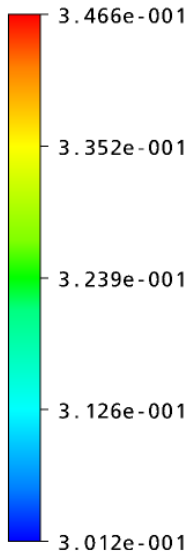

$\left[\mathrm{kg} \mathrm{m} \mathrm{m}^{\wedge}-3\right]$

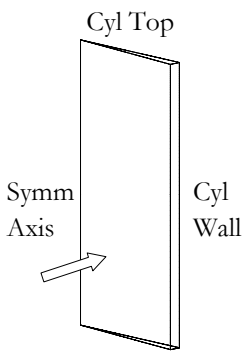

Piston

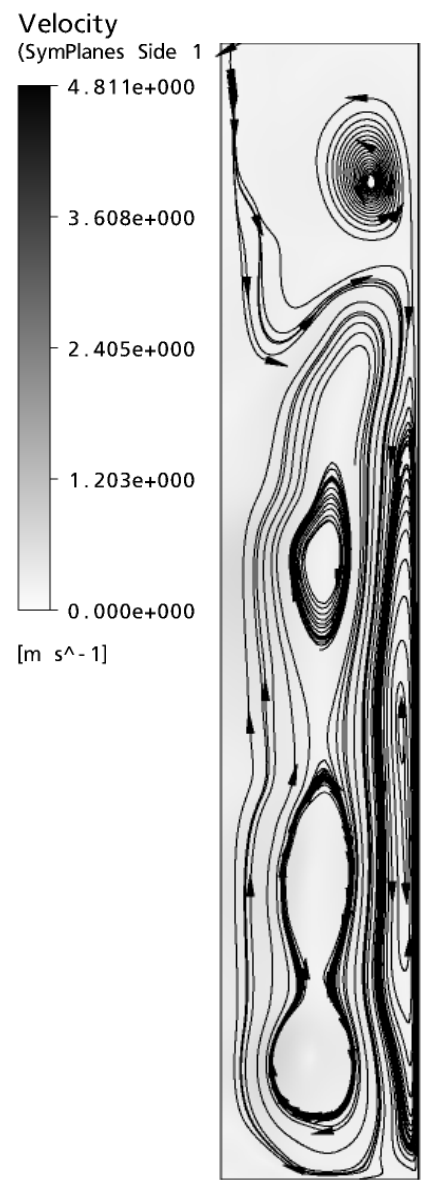

Figure 6.15: Gas streamlines and velocity field with piston in the BDC position
Figure 6.14: Typical gas density distribution, $80{ }^{\circ} \mathrm{C} A$ after TDC

be observed in the flow domains for runs discussed above (Figure 6.I I $5^{43}$ ), no developed turbulence is actually present here for any of the set operating frequencies. This is obvious from the turbulence parameters (eddy-viscosity, Reynolds number), and is actually in contrast with the conclusions reported in the work of Kornhauser [2]. The computed eddy frequency for the fast runs is three orders of magnitude smaller than the dynamic (molecular); whereas in the developed turbulent pipe flows it is three orders higher than the dynamic viscosity $[\boldsymbol{B}]$. At the same time, the Reynolds number peaks at $\sim 3000$ for the

43 The left vertical border in Figure 6.I5 represents the symmetry axis of the domain 2-D snapshot, the right is the cylinder wall, upper horizontal is the cylinder top and lower the piston. More on the development of fluid-flow structures is presented earlier, in Chapter 5. 
short-duration moment of maximum piston velocity. There are no published studies on critical Reynolds numbers in this type of geometries; but it is well established that for the pipe flows the turbulence is ensured only for $\mathrm{Re}>\mathrm{IO}^{4}$. Nevertheless, a viscous shear layer and a bulk core in inertial motion can be distinguished here as well, and this directly influences the formation of zones with highly segregated temperature profiles. During the high-speed runs, the shear layer thickness is much smaller than the characteristic length the piston diameter. In the slow runs though, the shear layer grows to the order of magnitude of the piston diameter. As mentioned, in this section the analytical analysis on the development and thickness of the thermal boundary layers are presented. Analytical results are evaluated on the results of the CFXII.O numerical simulations described in subchapters 6.2 and 6.3. The results for two different piston-cylinder geometries and a range of simulated operating conditions are taken as a validation database.

\subsubsection{Numerical Results}

The numerical models and the set of operating conditions for the numerical simulations are the two gas springs described in subchapter 6.2, over the range of operating frequencies: 2, IO, IOO, 500, IOOO and I500 RPM. This was seen as a good validation base for the investigation of the boundary layer development as well.

Although using the same numerical models, for the investigation of the temperature profiles in scope of this subchapter, post-processing of the simulations was performed in extended detail. The gas temperatures are sampled at three fixed axial heights between the TDC position and the cylinder top, and in the moment of the maximum piston velocities in the cycle $\left(78.7^{\circ}\right.$ and $79.8^{\circ}$ before and after the top dead centre, for the MIT and UT geometry, respectively). Data is sampled at every crossing node along the three horizontal lines, i.e. three axial radii. Typical produced radial temperature profiles are displayed in Figure 6.I6 and Figure 6.I744. As visible in the figures, as the frequency of the compression process rises, the forming and narrowing of the boundary layers can be observed. For the slow runs - 2\&IO RPM, the speed of the temperature propagation (which follows the heat and mass transfer in the gas) is large enough compared to the axial velocity of the gas, resulting in the Gaussian-like temperature profiles. As the piston speed grows, two regions in the compressed (expanded) volume appear: the viscous wall-affected boundary layer and the undisturbed turbulent-like core. Thermal and velocity boundary layers are formed as a consequence of the finite thermal conductivity of the gas and the finite value of the molecular viscosity, which, together with the no-slip condition at the walls and the time-scale of the momentum introduced by the piston displacement,

\footnotetext{
44 Heights of the sampling planes referred in these plots are on the axial TDC centre-line: 38.I mm and $26 \mathrm{~mm}$ below the cylinder top for the MIT and UT geometry, respectively.
} 

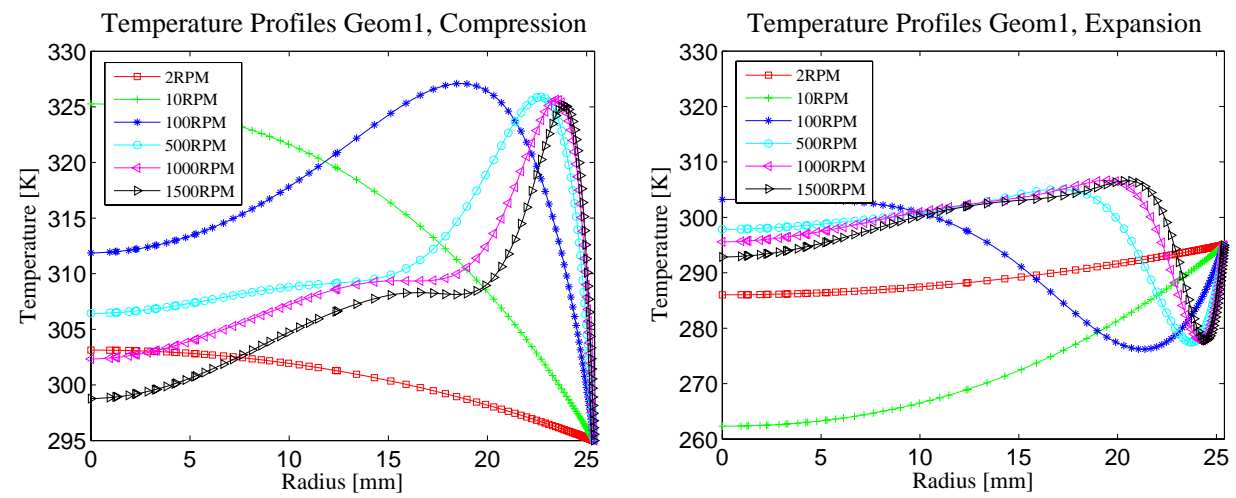

Figure 6.16: Temperature profiles in the compressed gas for the MIT gas spring, $101{ }^{\circ} \mathrm{CA}$ (left) and $259^{\circ} \mathrm{CA}$ (right)
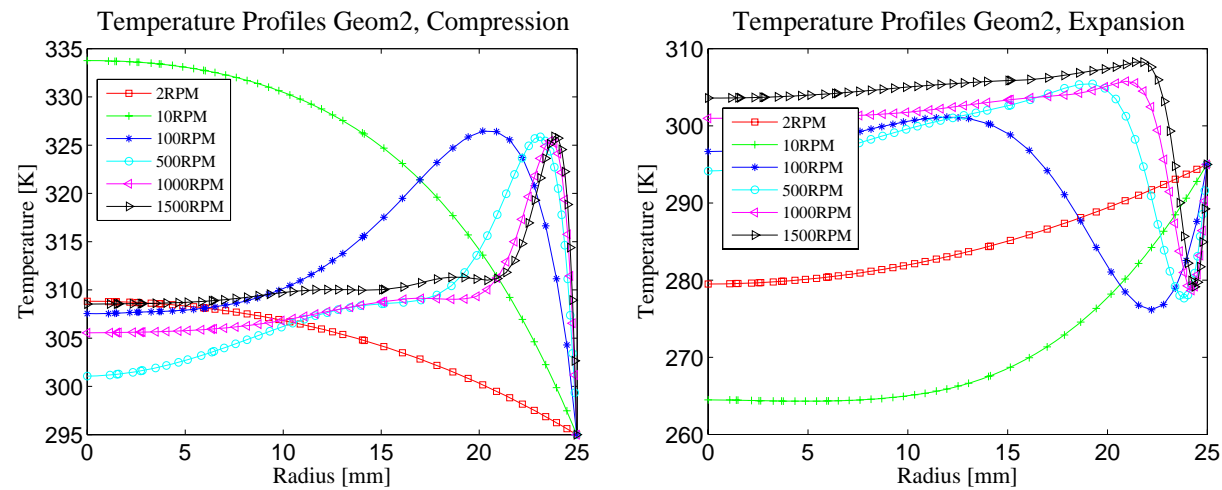

Figure 6.17: Temperature profiles in the compressed gas for the UT gas spring, $100{ }^{\circ} \mathrm{CA}$ (left) and $260^{\circ} \mathrm{CA}$ (right)

limits the radial homogenization. In the fast runs, the turbulent-like core is basically the bulk gas uniformly stretched or compressed in the axial direction.

\subsubsection{Analytical Correlation and Results}

Lyford-Pike and Heywood [5I] developed an analytical expression for the growth of the thermal boundary layers at the cylinder walls in a spark-ignition engine. They used a dimensional analysis and correlated the developed boundary layer thickness during the expansion process in a fitted form: 


$$
\delta_{\mathrm{T}}=0.6 \sqrt{\alpha t} R e^{0.2},
$$

with $\delta_{T}$ the thermal boundary layer thickness, $\alpha=\frac{k}{\rho c_{p}}$ the thermal diffusivity, $t$ the elapsed time from the TDC, and $R e=\frac{\rho v x_{0}}{\mu}$. The Reynolds number is here defined with $x_{0}-$ the distance from the sampling point to the cylinder top as the characteristic length, and velocity at the sampling point evaluated by $v=v_{p} \frac{x_{0}}{x}$. $v_{p}$ here is the piston velocity and $x$ is the distance between the piston crown and the cylinder top. The properties of the gas were evaluated for the cycle mean of the bulk gas and the wall temperature.

Lyford-Pike and Heywood determined the boundary layer thickness optically using a Schlieren visualisation system, sensitive to the density gradients. The measurements of the thickness of the boundary layers were then made from obtained photographs and movies during the expansion stroke. Since no such system was present in this study, numerical simulations are used here to determine the thermal boundary layer thickness ${ }^{45}$.

In order to discuss the boundary layer thickness correlations, first a criterion needs to be set for what should be regarded to as the actual boundary layer, i.e. where the transition point between the wall-affected and undisturbed region is. As a sensible choice, and also generally corresponding to the density-indicated visual method presented in $[\mathbf{5 I}]$, it is here proposed to adopt the transition region at the point where the temperature gradient $d T$ / $d x$, from the gas core towards the wall, is increased by $25 \%$. This criterion is specifically presented in Figure 6.I8, and correlates to the two discussed segregated

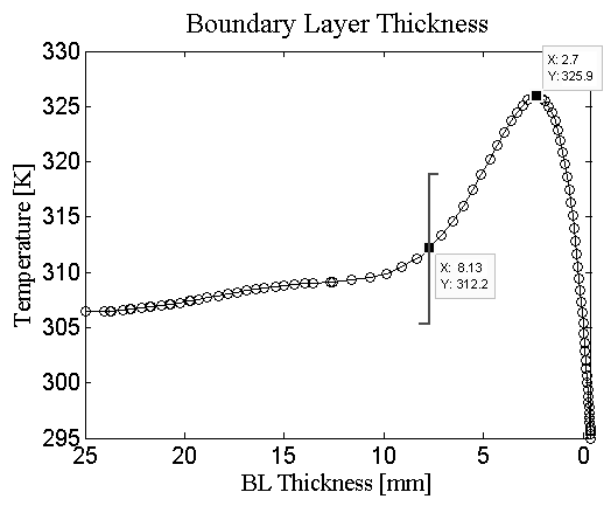

Figure 6.18: Boundary layer thickness

45 Although a visualisation of a numerically obtained density field is presented in Figure 6.I4. 


\begin{tabular}{cccc}
\hline $\mathbf{x}_{\mathbf{0}}[\mathrm{mm}]$ & Position I & Position 2 & Position 3 \\
\hline \hline Kornhauser & I9.05 & $38 . \mathrm{I}$ & $57 . \mathrm{I} 5$ \\
UT setup & $\mathrm{I} 3$ & 26 & 39 \\
\hline
\end{tabular}

Table 6.3: Gas temperature sampling positions, measured from the cylinder top

regions: of the steep near-wall boundary layers and the more uniform central region of the compressed volume discussed with Figure 6.I6 and Figure 6.I7.

It is also very interesting to point out the shape of the boundary layer: through all the here presented runs, the thermal profiles in the gas at the walls always follow a relation by which in this way set transition thickness equals very close to three times the radial distance from the wall to the peak-temperature in the boundary layer (in Figure 6.I8: $x_{B L}=8.13 \mathrm{~mm}$ versus $x_{T_{\max }}=2.7 \mathrm{~mm}$ ).

The sampling locations in numerical simulations $-x_{0}$ are specified in Table 6.3, as measured from the cylinder top. They were generally selected to sample 3 points in the unswept, 'dead' volume. Results for the boundary layer thicknesses (radial temperature profiles displayed in Figure 6.I6 and Figure 6.I7) are presented in Figure 6.I9. For operating frequencies 2 and IO RPM the boundary layers are not recognized (in agreement to the presented discussion for the slower runs) and will not further be taken in consideration. The thermal boundary layer thickness, for the rest of the operating spectrum, for the compression stroke ranges from approximately $20 \mathrm{~mm}$ for IOO RPM, to $4 \mathrm{~mm}$ for I500 RPM. For the expansion stroke, boundary layer thicknesses are somewhat smaller: from $13 \mathrm{~mm}$ for IOO RPM to $2 \mathrm{~mm}$ for I 500 RPM.

The performance of the Lyford-Pike and Heywood expression was examined as a next step. Since it is, in excess to their work, here proposed to extend correlations of the boundary layer thickness to both compression and expansion strokes (only expansion discussed in $[\mathbf{5 I}]$ ), the elapsed time in Equation (6.42) was corrected to start counting when the piston passes BDC for the compression stroke, and for the expansion stroke when it passes TDC, as before. The results for the fit at sampling position 2 (axially central in the 'dead' space) and both modelled geometries are displayed in Figure 6.20.

From the plotted analytical results it is obvious that the model does not perform satisfactorily. The relation in Equation(6.42) was in $[\mathbf{5 I}]$ fitted for the expansion stroke, two operating speeds: I380 and 8I5 RPM, bore/stroke ratio: 82.6/I I $4.3 \mathrm{~mm}$ and a higher compression ratio: $\mathrm{r}=4.8$, thus similar to the here investigated gas springs. Also, although 

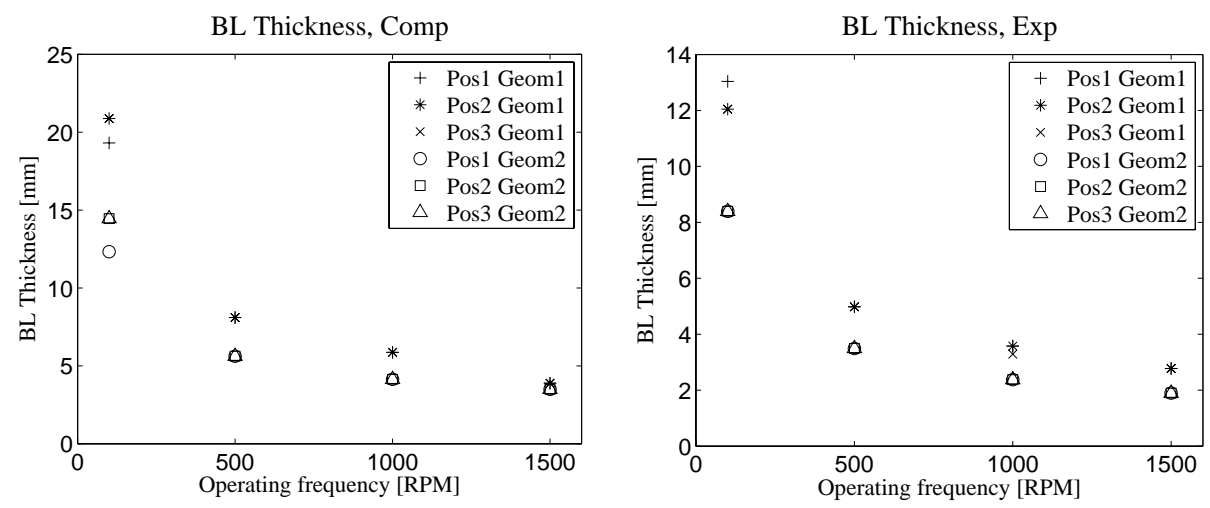

Figure 6.19: Boundary layer thickness for compression (left) and expansion stroke (right), 2 different geometries and 3 sampling positions
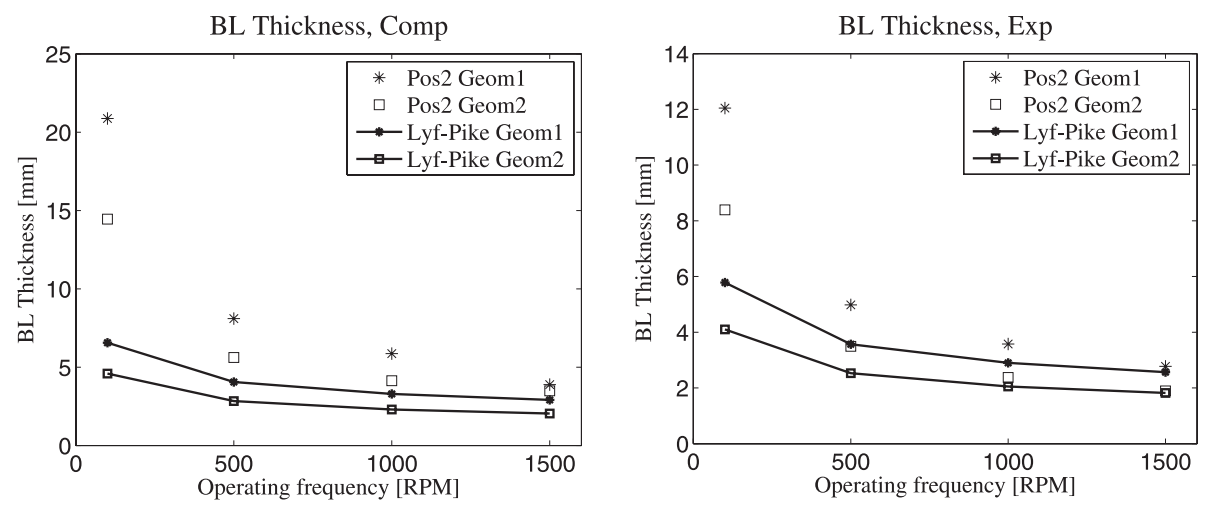

Figure 6.20: Boundary layer thickness at Position 2 and the analytical fit by Lyford-Pike and Heywood

developed for the spark-ignition engine with the intake and exhaust valves and with air as the working fluid, Equation (6.42) was derived for the expansion stroke when the valves are closed, and therefore the observed large discrepancy does come surprising. Nevertheless, care has to be taken that the flow formation processes (also discussed in subchapter 5.4) are different in a valveless gas spring machine and an intake/exhaust flow influenced construction such as one observed in $[\mathbf{5} \boldsymbol{I}]$.

It was for this reason decided to attempt to correct Equation (6.42), and investigate the scaling factors other than presented by Lyford-Pike and Heywood, that might be important for the phenomena observed in the gas springs in this research. First obvious 

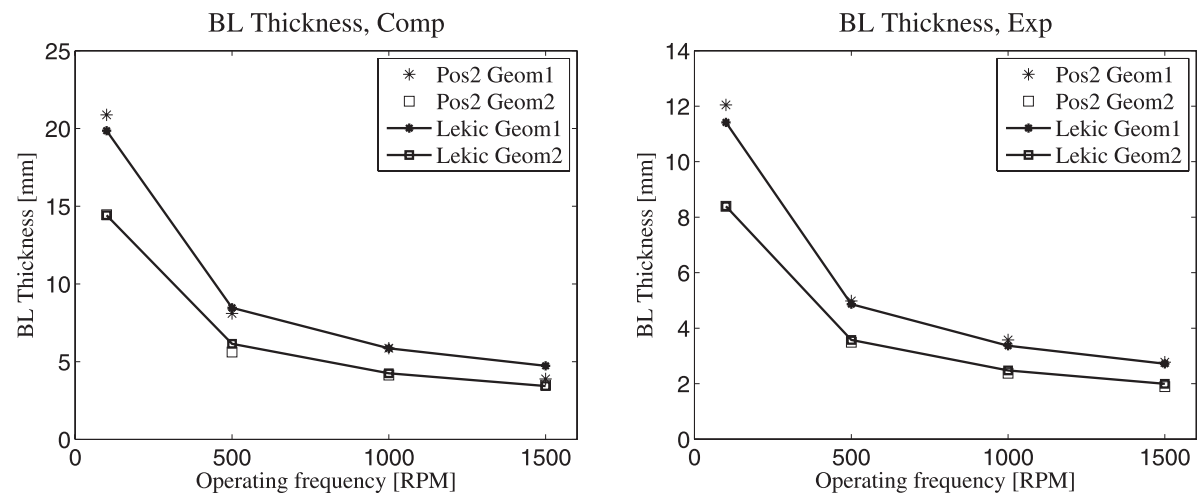

Figure 6.21: Boundary layer thickness at Position 2 and the analytical fit by Lekic

BL Thickness, Comp

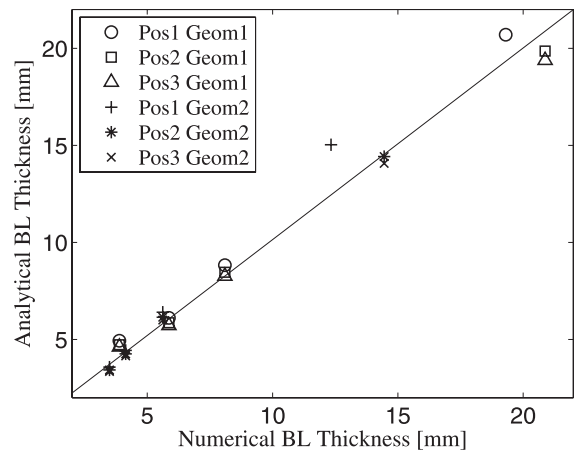

BL Thickness, Exp

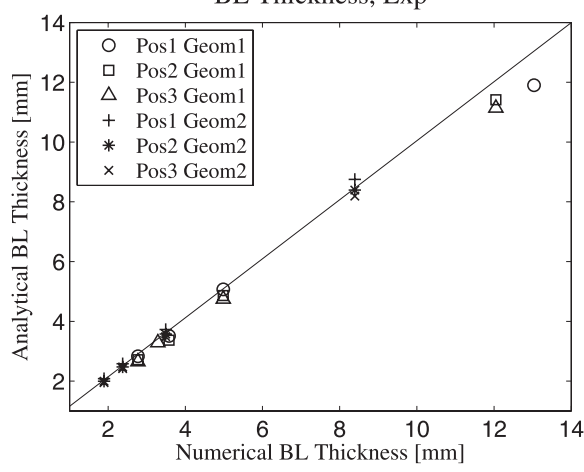

Figure 6.22: Performance of the proposed analytical correlation; boundary layer thickness over the full investigated range of operating conditions, compression (left), and expansion stroke (right)

correction was introducing a negative influence of the gas velocity through the Reynolds number; it is intuitively sensible that the boundary layer thickness will decrease with faster operation, and in Equation (6.42) the power of $R e$ is positive. Further, the bore/stroke ratio influence should be included in the expression, since the geometry of a convoluted volume must significantly influence the dynamic formation of fluid structures. Finally, the empirical coefficients were fitted, and this resulted in the following expressions:

$$
\delta_{\mathrm{T}, \mathrm{comp}}=4.6 \sqrt{\alpha \cdot t} \cdot \frac{1}{\left(\operatorname{Re} \cdot \frac{S}{D}\right)^{0.03}}=4.6 \sqrt{\alpha \cdot t} \cdot \frac{1}{\operatorname{Re}_{S}^{0.03}}
$$

for the compression stroke, and 


$$
\delta_{\mathrm{T}, \exp }=3 \sqrt{\alpha \cdot t} \cdot \frac{1}{\left(R e \cdot \frac{S}{D}\right)^{0.03}}=3 \sqrt{\alpha \cdot t} \cdot \frac{1}{R e_{S}^{0.03}}
$$

for the expansion stroke. In Figure 6.2I the improved results for the sampling position 2 are displayed. This is extended in Figure 6.22, where the results for all three sampling positions, and thus all investigated cases are summarized and compared to the numerically obtained values. The analytical models correlate the data very closely for all tested cases with exception of the results sampled at Position I for the compression stroke, where the thickness is overestimated by approximately $25 \%$ of the numerical value ${ }^{46}$. Nevertheless, the data is well matched for a wide range of settings, and it can be concluded that the presented analytical model performs well.

\subsubsection{Summary}

In view of the relevance to the thermodynamic processes and gas-wall heat transfer, the depth of penetration of the thermal boundary layers at the cylinder walls of a gas spring is analyzed. Results for temperature profiles obtained from the numerical simulations are taken as a validation source for the analytical correlations. The analytical expression derived by Lyford-Pike and Heywood [5I], modelling the thermal boundary layer thickness at the cylinder walls of a spark-ignition engine during the expansion stroke is examined and taken as the base-line. After poor comparison, it is corrected with inclusion of a factor to compensate for the negative influence of the gas velocity on the boundary layer thickness, the bore-to-stroke ratio, and extended with an added expression to model both expansion and compression strokes. The proposed correlations fit the numerical data adequately for all tested cases: for two different helium gas spring geometries with two different initial charge pressures, a range of operating frequencies and the compression and expansion stroke. It can be concluded that the presented model is a good approximation and can be used generically for the similar investigations.

\footnotetext{
${ }^{46}$ Position I is closest to the cylinder top, where the gas velocities are strongly damped through the entire compression process. For the slower run (8I5 RPM), Lyford-Pike and Heywood report the boundary layer thickness at the cylinder top and piston to be two to three times thicker than for points along the walls.
} 


\section{CHAPTER
RECONCLUSIONS AND}

As every well time-managed project, this one also comes to its end. This last chapter summarizes the conclusions drawn from the presented work, assesses the achievements and fulfilment of goals stated at the beginning of the thesis, and presents the envisioned directions for potential improvements and continued efforts on the subject.

\section{I Conclusions}

At the beginning of this thesis, in Chapter I, the goals of this project are summarized and put into the context of the project setting and pursue for definition of the design rules for the improved next-generation gas compressors (and ideally more generally extended to wider reciprocating machinery). In this chapter we want to assess how much of those goals has been achieved and what knowledge was gained along the way. If we translate those goals into concise questions, such a list would look something like this:

\section{Questions}

- Have we designed an experimental setup, that is capable of accurate and fast measurements of physical parameters of concern, for the research of thermal phenomena in piston-cylinder gas springs? Have we created a measurement database, that can be used for thermodynamic analyses and validation of the related numerical and analytical models? 
- Have we created numerical models, to simulate the operation of the experimental gas spring and capture the physical properties and processes in its fluid domains, over a span of compression ratios, operating conditions and frequencies?

- Have we assessed the available analytical expressions and models applicable to the investigated problems, and evaluated them on the obtained experimental and numerical results?

- Did we learn more, did we identify windows for improvements, and how can this research be continued and supplemented?

Discussions on the presented problems and their solutions, observations and effectiveness of the proposed methods are already presented at the ends of concerned sections or subchapters and finally overviewed at the end of every respective chapter. These are elaborate and given in details, sometimes numbers and more specific information; for the sake of compactness and a convenient overview, here they are structured so to directly answer the questions posed above.

\section{Answers}

- The experimental gas spring created at the University of Twente is made for a wide range of thermodynamical investigations and experimental measurements of concern. Its modular design allows for various adaptations, including any desired compression ratio, linear frequency modulation $(\mathrm{O}-5 \mathrm{O} \mathrm{Hz}$ ), cooling of the compressing section etc. Diverse sensing instrumentation is installed, including four robust and precise pressure transducers, two miniature gas thermocouples, seven surface and in-wall thermocouples for thermal mapping of the solid parts and a crank-angle encoder. The data acquisition system is capable of the maximum sampling rate of 500 kilo-samples per second and sampling on 32 input channels. Post-processing algorithms are developed for calibration, data noise reduction, data bucketing and convenient presentation.

A database of measured properties is created and consists of the described measured parameters, for the compression ratio 2 gas spring: operating frequencies of $2,5,10,15,20$ and $25 \mathrm{~Hz}, 3$ charge pressures (give numbers), and the compression ratio 8 gas spring: the same range of operating frequencies and one baseline gas pressure $\left(\phi_{B D C}=\sim 0.5\right.$ bar). Accurate direct measurements of gas pressures at three locations, surface and in-wall temperatures of the piston and cylinder wall and head are recorded, while transient gas temperature could not be correctly captured in spite of extremely small miniature gas thermocouples. Nevertheless, for this purpose a method to estimate the gas temperatures and correlated mass of the captured gas from the recorded signal of the installed thermocouples was developed. 
- Numerical models for all geometries (MIT and UT, compression ratios 2 and 8) are created in commercial ANSYS CFXII.O software. The computational fluid domains are represented by $5^{\circ}$ slices and optimized with respect to mesh refinement and temporal resolution; models are second-order accurate in both space and time. Boundary and initial conditions and operating frequencies can be set according to the values measured in the experiments. Numerical models are using deformable meshes; no re-meshing is needed. DNS approach is adopted and no approximating turbulence models are used. Using these numerical models virtually all physical properties can be simulated and conveniently postprocessed; for the sake of this research analyzed are gas pressure, gas temperature, gas density, wall heat flux, streamlines and velocities in the compressed gas, mass of the compressed gas and internal energy balance over the computational domain.

For the MIT runs, the simulations performed very satisfactory, both gas pressure and wall heat flux are very accurately simulated and the maximal imposed error in the pressure results is $\sim 2.5 \%$ of the gas pressure amplitude. For the simulations of the UT setup, where the physical construction is such that the leakage past the compressing piston is partly allowed, numerical models are extended with a mass source (sink) in the vicinity of the piston-cylinder contact, in order to capture the mass transfer in the experiments. Since the exact clearance in the experimental setup is not measured, iterative modelling approach led to a value of the radial clearance of $19.5 \mu \mathrm{m}$ for compression ratio 2 , and has shown very good results, with less than $2 \%$ of error in the pressure results over the entire operating frequency range, as compared to measurements. For compression ratio 8, another iterative cycle had to be performed, and gave best results for the radial clearance of $4 \mu \mathrm{m}$. This discrepancy is suspected to originate from the imprecise assembling procedure and/or the secondary motion of the piston. The error in the pressure simulations for compression ratio 8 is then no more than $2.7 \%$ for all investigated operating frequencies. Experimental evaluation of the heat flux is not justified for the UT runs (heat flux evaluation method explained in Appendix B), and thus the performance of the numerical simulations on the wall heat flux results could not be assessed.

To conclude, created numerical models perform good and are well capable of capturing the observed physical properties and processes. Simulations are robust and allow for various settings for both numerical solver and physical parameters. Post-processing is done in the same CFD package and is capable of performing a wide range of analyses. Validation of the numerical models on the experimental database is somewhat hampered due to the uncertainty in setting of some physical parameters, originating from the construction of the experimental setup.

- Vast literature was analyzed and several analytical expressions carefully assessed on the available data. As three somewhat separate phenomena were investigated, different success was accordingly acquired: 
The non-dimensional variables, initially presented by Lee [45] and Cooke-Yarborough and Ryden [46], and then reviewed by Kornhauser [2], [38], [47], correlate cyclic hysteresis heat loss, pressure wave magnitude and pressure wave phase shift reasonably well over the investigated range of operating conditions, for the gas springs with compression ratio 2 (Equations (6.I) to (6.6)). The phenomena are shown to be fairly well predicted over the investigated range of operating speeds, initial pressures and geometries. Nevertheless, empirical correction factors for the expressions by Kornhauser are introduced and show improvements (Equations (6.I7), (6.I8) and (6.I9)).

The analytical expression for the complex Nusselt number, derived by Lee and corrected by Kornhauser (Equation (6.32)), shows to be reasonably successful but still not sufficiently accurate. A correction in parameterization presented in Equation (6.40) somewhat reduces the analytical modelling errors, but still allows for the under-prediction of the heat flux amplitude of $\sim 25 \%$, and a phase shift of $\sim$ IO ${ }^{\circ} \mathrm{CA}$. In another approach, the real and imaginary parts of the complex Nusselt number show a power-law relation to the oscillation Péclet number and can be expressed in the form $N u_{r, i}=c_{1} P e_{\omega}^{c_{2}}+c_{3}$, with empirical coefficients $c_{1}$ to $c_{3}$. The heat flux calculated in this way predicts the experimental data over a wide range of operating conditions well in amplitude, but still introduces phase errors of the same magnitude as the other presented analytical models.

Depth of penetration of the thermal boundary layers at the cylinder walls of a gas spring is also investigated. Expression derived by Lyford-Pike and Heywood [5I $]$ is taken as the baseline; in its original form it models the thermal boundary layer thickness at the walls of a spark-ignition engine during the expansion stroke. After poor comparison, it is corrected to include the influence of gas velocity, bore-to-stroke ratio, and supplemented to model both expansion and compression stroke. The proposed correlations fit the numerical data adequately for all tested cases: for two different helium gas spring geometries with two different initial charge pressures, a range of operating frequencies and the compression and expansion stroke.

\subsection{Recommendations}

- Answer to the last question is optimistic. Presented results definitely add to the capabilities of scientific research in the field of thermal analyses of reciprocating compression machines. Valuable experimental setup is built and can be used for creating wider and more repeatable measurement and validation databases. ANSYS CFXII.o has proven to be a very robust and user-friendly finite volume numerical package, and the created models show full potential for the numerical modelling in the process evaluation and prediction of physical parameters of interest for the reciprocating machinery. 
Presented analytical expressions show modest generality, but still can be used as initial estimators for some generic considerations.

Work on this topic will hopefully be continued by future new research initiatives, at the University of Twente or elsewhere. Continued efforts should concentrate in several directions:

- A heat flux calculation routine should be developed to directly evaluate the heat flux from the surface and in-wall thermocouple measurements. This will allow for full validation of the numerical models and direct comparison of wall heat flux simulations;

- In parallel, numerical work should be continued on the two- and three-domain simulations (namely the compressed fluid, surrounding walls and the cooling channels), extending the numerical modelling potential to the thermal mapping of the surrounding solid walls and in-wall temperature distribution, which is crucial for the direct prediction of the distortions of geometries and material properties of the concerned machine parts;

- Models should be examined on inclusion of the charge and exhaust flows met in compressors. This way the modelling capabilities can be extended from Stirling-type machines to wider piston-cylinder constructions;

- Any future efforts need to be continuously supplemented with the results of the thermomechanical and tribological considerations, regarding the effects of frictional heating, surface degradation, wear and fatigue on the thermal processes;

- Improved models should finally be employed as modelling tools in the industrial development processes of modern compressor constructions in reciprocating machinery. 



\section{LIST OF REFERENCES}

[I] Pawel Owczarek, "On the design of lubricant free piston compressors," University of Twente, Enschede, PhD Thesis 2010.

[2] A.A. Kornhauser, "Gas-Wall Heat Transfer During Compression and Expansion," Massachusetts Institute of Technology, PhD Thesis I989.

[3] The Engineering ToolBox. Coefficients of Linear Expansion. [Online]. http://www.engineeringtoolbox.com/linear-expansion-coefficients-d_95.html

[4] Jacob Nanigian and Nanigian Daniel, "A Unique Thermocouple to Measure The Temperatures of Squibs, Igniters, Propellants and Rocket Nozzles," in Sensors for Propulsion Measurement Applications. Proceedings of SPIE, vol. 6222, 2006, pp. 6222-3.

[5] B Lawton, "Effect of compression and expansion on instantaneous heat transfer in reciprocating internal combustion engines," MIMechE Paper, vol. 20I, no. A3, I987.

[6] Jan A Gatowski, Mark K Smith, and Alex C Alkidas, "An Experimental Investigation of Surface Thermometry and Heat Flux," Experimental Thermal and Fluid Science, pp. 280-292, I989.

[7] William Navidi, Statistics for Engineers and Scientists.: McGraw-Hill, 2006.

[8] ANSYS Europe Ltd. (2005) ANSYS CFX-Solver, Release: I0.0: Theory. 
[9] ANSYS Europe Ltd. (2006, December) ANSYS CFX-Solver Theory Guide.

[Io] R. Byron Bird, Warren E. Stewart, and Edwin N. Lightfoot, Transport Phenomena, Second Edition:: John Wiley \& Sons Inc., 2002.

[II] Jr. John D. Anderson, Computational Fluid Dynamics - The basics with applications.: McGraw-Hill, Inc., I995.

[I2] Wikipedia. Ideal gas law. [Online]. http://en.wikipedia.org/wiki/Ideal_gas_equation

[I3] Wikipedia. Fluid Mechanics. [Online].

http://en.wikipedia.org/wiki/Fluid_mechanics\#The_continuum_hypothesis

[I4] H.E. Velten, "Wall heat transfer in a lubricant-free piston compressor," University of Twente, Master's Thesis 2009.

[I5] Pieter Wesseling, Principles of Computational Fluid Dynamics.: Springer series in computational mathematics, 2000.

[16] D.C. Wilcox, Turbulence Modelling for CFD. California, USA: DCW Industries, I994.

[17] S.B. Pope, Turbulent flows.: Cambridge University Press, 2000.

[i8] U. Lekić and J.B.W Kok, "Heat Flows in Piston Compressors," in 5th EUROTHERM, 2008, FCV_4.

[I9] Wikipedia. Leibniz Integral Rule. [Online].

http://en.wikipedia.org/wiki/Leibniz_integral_rule

[20] P. D. Thomas and C. K. Lombard, "The Geometric Conservation Law - A link between finite-difference and finite-volume methods of flow computation on moving grids," in Fluid and Plasma Dynamics Conference, Seattle, I978, p. I5.

[2I] Henson Van Emden. (2009, April) An Algebraic Multigrid Tutorial. [Online]. https://computation.llnl.gov/casc/linear_solvers/talks/AMG_TUT_PPT/sldooi.h tm

[22] J.H. Ferziger and M. Peric, Computational Methods for Fluid Dynamics, 3rd ed. Berlin: Springer-Verlag, 200I. 
[23] ANSYS Europe Ltd. (2006, December) ANSYS CFX-Solver Modeling Guide.

[24] Richard Stone, Introduction to Internal Combustion Engines, 3rd ed. London: MacMillan Press Ltd, I999.

[25] Adair et al., "Instantaneous heat transfer to the cylinder wall in reciprocating compressors," in 1972 Purdue Compressor Technology Conference, I972.

[26] W.J.D. Annand and D. Pinfold, "Heat Transfer in the Cylinder of a Motored Reciprocating Engine," Society of Automotive Engineers, SAE Paper 800457, I980.

[27] H.G. Jak, "Heat Transfer in a Gas Spring," University of Twente, M.Sc.Thesis $200 I$.

[28] Paul B. Bailey, Michael W. Dadd, C. Richard Stone, Jaime S. Reed, and Thomas M. Davis, "Gas Spring Losses in Linear Clearance-Seal Compressors," in International Cryocooler Conference, 2007.

[29] D.W. Dickey and Ch.D. Wood, "Analytical and experimental investigation of ringless piston concept," Gas Research Institute, Technical report I988.

[30] Lewis F. Richardson, Weather Prediction by Numerical Process.: Cambridge Press, I922.

[3I] P.A. Davidson, Turbulence: An Introduction for Scientists and Engineers: Oxford University Press, 2004.

[32] John E Hesselgreaves, Compact Heat Exchangers: Selection, Design and Operation: Elsevier Science \& Technology Books, 200I.

[33] Yunus A. Çengel and Robert H. Turner, Fundamentals of Thermal-Fluid Sciences, International Edition ed.: McGraw-Hill Higher Education, 200I.

[34] Đ. Kozić, B. Vasiljević, and V. Bekavac, Priručnik Za Termodinamiku, Ioth ed.: Mašinski Fakultet Univerziteta u Beogradu, 200I.

[35] M.W. Dadd, "Acoustic thermometry in gasses using pulse techniques," High Temperature Technology, vol. I, no. 6, pp. 333-342, November I983.

[36] E.J. Lyford-Pike and J.B. Heywood, "Thermal boundary layer thickness in the cylinder pf a spark-ignition engine," Int. J. Heat Mass Transfer, vol. 27, pp. I873-I878, I984. 
[37] Kendall A. Atkinson, An Introduction to Numerical Analysis , 2nd ed.: John Wiley \& Sons, I989.

[38] A. A. Kornhauser and Jr. J. L. Smith, "The Effects of Heat Transfer on Gas Spring Performance," Journal of Energy Resources Technology, vol. I I5, pp. 70-75, March 1993.

[39] H. Pfriem, "Periodic Heat Transfer at Small Pressure Fluctuations," NACA, no. TM-I048, I943.

[40] W.J.D. Annand, "Heat Transfer in the Cylinders of Reciprocating Internal Combustion Engines," Proceedings of the Institution of Mechanical Engineers, vol. I77, no. 36, pp. 973-996, 1963.

[4I] G. Woschni, "A Universally Applicable Equation for the Instantaneous Heat Transfer Coefficient in the Internal Combustion Engine," Society of Automotive Engineers, SAE Paper No. 6709311967.

[42] R.W. Jr. Breckenridge, T.P. Heuchling, and R.W. Jr. Moore, "Rotary-Reciprocating Cryogenic Refrigeration Studies, Part I, Analysis," Air Force Flight Dynamics Laboratory, AFFDL-TR-7I-II5, I97I.

[43] H.B. Faulkner and J.L. Jr. Smith, "Heat Transfer During Compression and Expansion in Reciprocating Gas Handling Machinery," in 18th Intersociety Energy Conversion Engineering Conference, I983, pp. 724-730.

[44] H.B. Faulkner, "An Investigation of Instantaneous Heat Transfer during Compression and Expansion in Reciprocating Gas Handling Equipment," Dept. of Mech. Eng., Massachusetts Institute of Technology, PhD Thesis I983.

[45] K.P. Lee, "A Simplistic Model of Cyclic Heat Transfer Phenomena in Closed Spaces," in 18 th IECEC, I983, pp. 720-730.

[46] E.H. Cooke-Yarborough and D.J. Ryden, "Mechanical Power Losses Caused by Imperfect Heat Transfer in a Nearly-isothermal Stirling Engine," in 20th Intersociety Energy Conversion Engineering Conference, I985, pp. 3.307-3.3I2.

[47] A.A. Kornhauser and J.L. Smith, "A Comparison of Cylinder Heat Transfer Expressions Based on prediction of Gas Spring Hysteresis Loss," Fluid Flow in Heat Transfer and Reciprocating Machinery, pp. 89-96, I987. 
[48] J.D. Annand and D. Pinfold, "Heat Transfer in the Cylinder of a Motored Reciprocating Engine," SAE Paper, no. 800457, I980.

[49] A. A. Kornhauser and J. L. Jr. Smith, "Application of a Complex Nusselt Number to Heat Transfer During Compression and Expansion," ASME, vol. iा6, pp. 536542, August I994.

[50] D.H. Menzel, Fundamental Formulas of Physics.: Courier Dover Publications, I960, vol. I.

[5I] E.J, and Heywood, J.B. Lyford-Pike, "Thermal boundary layer thickness in the cylinder pf a spark-ignition engine," Int. J. Heat Mass Transfer, vol. 27, pp. I873-I878, I984.

[52] C.R. Wylie, Advanced Engineering Mathematics.: McGraw-Hill, I960.

[53] T.T. Wu and W.H. Hsieh, "Compression processes and performance analysis of a high-pressure reciprocating gas compressor," Journal of mechanical engineering science, vol. 210, pp. I53-I65, I996.

[54] D.R. Otis, "Thermal Losses in Gas-Charged Hydraulic Accumulators," in 8th Intersociety Energy Conversion Engineering Conference, I973, pp. 466-472.

[55] J.E. McCullough, "Exploratory Development of Gas Springs as Applied to RotaryReciprocating Cryogenic Coolers," Air Force Flight Dynamics Laboratory, AFFDLTR-67-62, I976.

[56] Falkovich Gregory and Katepalli R. Sreenivasan, "Lessons from Hydrodynamic Turbulence," Physics Today, vol. 59, no. 4, pp. 43-49, April 2006.

[57] G. Borman and K. Nishiwaki, "Internal Combustion Engine Heat Transfer," Progress in Energy and Combustion Science, vol. I3, no. I, pp. I-46, I987.

[58] Dmitri Kuzmin. Introduction to CFD. [Online]. http://www.mathematik.unidortmund.de/ $\mathrm{kuzmin} / \mathrm{cfdintro} / \mathrm{cfd} . \mathrm{html}$

[59] CFX-Online. [Online]. http://www.cfd-online.com/Wiki/Sutherland_law 

APPENDICES 



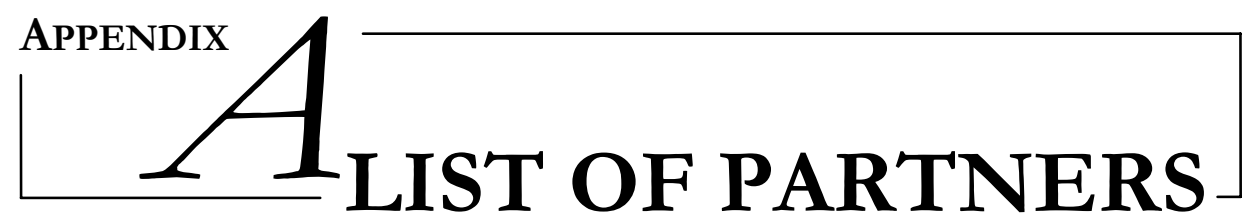

In this section an overview of the industrial partners and a short motivation for their involvement on the project "Design rules for close tolerance and lubricant free piston compressors" is given. Partners are sorted in alphabetical order.

- ANSYS Europe Ltd. The CFX Technical Services group in the UK is a department within the Fluids Business Unit of ANSYS Inc, which is responsible for the development and exploitation of the world-leading CFX software for Computational Fluid Dynamics (CFD). Its core customer base includes the Chemical and Process Industries, Power Generation, turbo-machinery, HVAC and Fire. It has well over IOOO licensees worldwide, in industry, research laboratories and academia, with its most prominent products CFX-4 to today's CFX-I3. Many of the staff have an international reputation in the development and application of CFD.

CFX forms the Fluids Business Unit of ANSYS Inc, based in Canonsburg, Pennsylvania, USA, a major developer of engineering software for stress analysis and mesh generation (ICEM). Together, the combined organisation has a formidable expertise in Engineering Analysis software for fluid flow and stress analysis. Within the UK operating company, ANSYS Europe Ltd, there are around 60 staff members, with expertise in core issues like mesh generation, turbulence modelling, stress analysis etc. CFX considers the application of Computational Fluid Dynamics linked to Computational Solid Mechanics as a research and development tool for piston compressors to be of great relevance to the performance and reliability of these machines. The further development of these computational tools with regard to moving mesh generation and to regimes of high compression ratios and for 
gaseous media at low temperature and with high diffusivity (like Helium) is important. CFX supported this development and the implementation in their CFX software very strongly.

- GEA Grasso is one of the leading manufacturers of innovative reciprocating compressors for refrigeration purposes. The head office is in 's Hertogenbosch in the Netherlands, with factories in the Netherlands and Germany. Grasso's reciprocating range includes over 50 types of compressors. Effort has been made to optimize them amongst others in the following aspects: good coefficient of performance at full and part load operation, high quality wear resistant parts, optimized lubrication, low vibration and noise level, high reliability. The refrigerant medium used can be for example NH3, Ri34a, $\mathrm{R} 404 \mathrm{~A}$ or R 507 . In order to avoid contamination of the refrigerant, carryover of lubricant has to be minimized. Vice versa, care has to be taken that refrigerant carry over will not deteriorate the performance of the lubricant. These problems are eliminated when a design can be used that is lubricant free. Essential aspect is that the reliability of the pistoncylinder system is at a very high level, to ensure smooth operation. Grasso works continuously on product improvement and innovation. This is supported by computer simulation models, computer aided design facilities and know-how in refrigeration technology. New designs are tested both in Grasso's laboratories and in field situations.

- Noble Products Twente B.V. and the daughter company Noble Products Germany GmbH NPG are engineering design profile companies that specialize in developing machine parts and machines from high performance materials. The main field in which both company are active is design parts based on kolsterised steel. Products like pumps, dosing system are supplied to a wide range of customers from chemical, food, pharmacy industry.

- Philips Domestic Appliances and Personal Care is interested in the utilisation of lubricant free PCC for a number of applications especially in pumping hot fluids and vapours for food preparation.

- Stirling Cryogenics \& Refrigeration B.V. (Stirling) is the world's leading innovator in stand-alone cooling systems. It provides solutions for gas and liquid processes at temperatures ranging from $200 \mathrm{~K}$ to $20 \mathrm{~K}$. For over half a century the company has been applying the (Philips) Stirling cycle cryogenerator technology, which enables liquid gases to be produced on-site. Stirling has developed a wide variety of applications like liquid gas production plants, process coolers, gas liquefiers, power coolers and cold cabinets, all based on the stand-alone Stirling cryogenerator technology.

Over 4,00O Stirling cooling systems are already installed worldwide. Customers include artificial insemination centres (livestock improvement), amusement parks, research \& 
development institutes, high-temperature super-conducting labs, hospitals, universities, observatories and logistic suppliers. Stirling develops and produces standard as well as customized equipment, and offers a full support package that includes installation, field service and spare parts.

The Stirling Consulting Group (SCG), a separate business unit, combines thermal knowhow with practical expertise, and offers thermodynamic consulting, design evaluation, design proposal and project management/ownership. Stirling believes that everyone who requires cold or cold gasses/liquids should be able to produce them themselves, wherever and whenever required, and independent of third-party suppliers. Stirling equipment is easy to integrate in (existing) processes, simple to operate, reliable, and extremely durable.

Stirling equipment uses highly efficient and simple technology. The straightforward construction means that reliability is designed in. New production techniques greatly decrease the frequency of maintenance intervals. Stirling equipment is built for long and reliable operation: the average lifespan of a Stirling system is 25 years! This really typifies Stirling: high-quality systems that continue to function exactly according to customer specifications.

- Thales Cryogenics - Netherlands. The former Cryogenic department of Signaal USFA, located in Eindhoven, The Netherlands and the company Cryotechnologies, located in Blagnac/Toulouse, France, are both member of the Thales Group and are operating since end 2000 under the same brand name THALES Cryogenics. These two separate companies have become a leading manufacturer of cryogenic equipment working closely together in development, production and commerce.

Thales Cryogenics manufactures high tech cooling equipment for application in the production of electronic components, silicon chips, laboratory research etc. Typical cooling is down to temperatures of $50 \mathrm{~K}$ and compressor input power $200 \mathrm{~W}$. On a yearly basis $\sim 4, \mathrm{OOO}$ units are produced. The process uses the (Philips) Stirling cycle principle with linear motor driven piston cylinder combinations. Reliable lubricant free operation under various temperature loads is of the ultimate importance. The proposed research will support this development. 



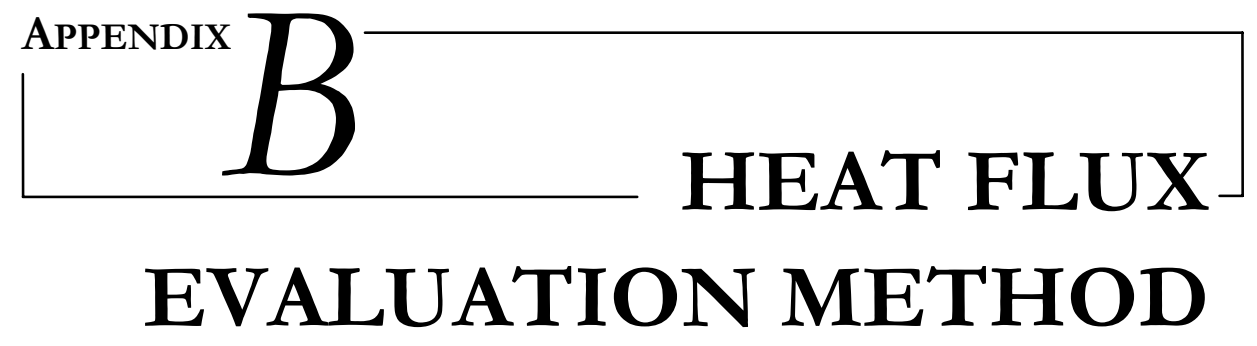

Unfortunately, during the course of this research, a method for direct evaluation of the heat transfer from the gas to, and through the surrounding walls of the gas springs was not developed. $V$ arious advanced equipment was installed in order to transiently measure the temperatures of the gas and the solids at several positions, but a generic algorithm that would correctly capture the temperature gradients in the walls and relate them to the dynamics of the process was still out of reach. Nevertheless, an indirect method, to evaluate a surface averaged heat flux, was proposed by Kornhauser [2], and adopted in this research as well. The method is based on the first law of thermodynamics and the gas equation of state, and the energy balances that correlate the other thermodynamic properties that could be measured accurately: the initial gas pressure, temperature and volume, and the instantaneous values of the compressed gas pressure and volume as functions of time.

The heat flux evaluation procedure that is referred to in multiple places in this thesis and initially proposed by Kornhauser [2] is presented by the below set of equations. The heat transfer rate was calculated from the pressure, volume and time data together with the gas equation of state and the first law of thermodynamics. The time derivatives were evaluated applying a five-point least squares fit model (Menzel [46]) to the time-dependent measured data. Since data was collected on a crank-angle basis rather than time basis, and, in first place for the slower runs, the angular frequency varied slightly over the cycle, the recorded 
data was translated to a time basis before differentiation and transferred back to a crankangle basis afterwards for plotting and comparison to the numerically obtained results.

From the initial values of pressure, temperature and volume of the gas, gas temperature at every instant of the cycle is calculated as:

$$
T_{i}=\frac{p_{i} V_{i}}{\frac{p_{\text {init }} V_{\text {init }}}{T_{\text {init }}}},
$$

for a constant mass of the compressed gas.

Time-derivatives of the gas temperature and volume at every instant of a compression cycle are then:

$$
\begin{aligned}
& \left(\frac{d T}{d t}\right)_{i}=\frac{5 \sum_{j=i-2}^{i+2} T_{j} t_{j}-\sum_{j=i-2}^{i+2} T_{j} \sum_{j=i-2}^{i+2} t_{j}}{5 \sum_{j=i-2}^{i+2} t_{j}^{2}-\left(\sum_{j=i-2}^{i+2} t_{j}\right)^{2}}, \\
& \left(\frac{d V}{d t}\right)_{i}=\frac{5 \sum_{j=i-2}^{i+2} V_{j} t_{j}-\sum_{j=i-2}^{i+2} V_{j} \sum_{j=i-2}^{i+2} t_{j}}{5 \sum_{j=i-2}^{i+2} t_{j}^{2}-\left(\sum_{j=i-2}^{i+2} t_{j}\right)^{2}} .
\end{aligned}
$$

The rate of change of internal energy is calculated as:

$$
\left(\frac{d U}{d t}\right)_{i}=m c_{v}\left(\frac{d T}{d t}\right)_{i}
$$

and the rate of heat transfer, defined as positive when heat is leaving the system:

$$
\dot{Q}_{\iota}=-\left[m c_{v}\left(\frac{d T}{d t}\right)_{i}+p_{i}\left(\frac{d V}{d t}\right)_{i}\right]
$$

Finally, the surface averaged wall heat flux at any time instant is then:

$$
\dot{q}_{l}=\frac{\dot{Q}_{l}}{A}
$$

evaluated over the entire heat transfer surface $A$. 


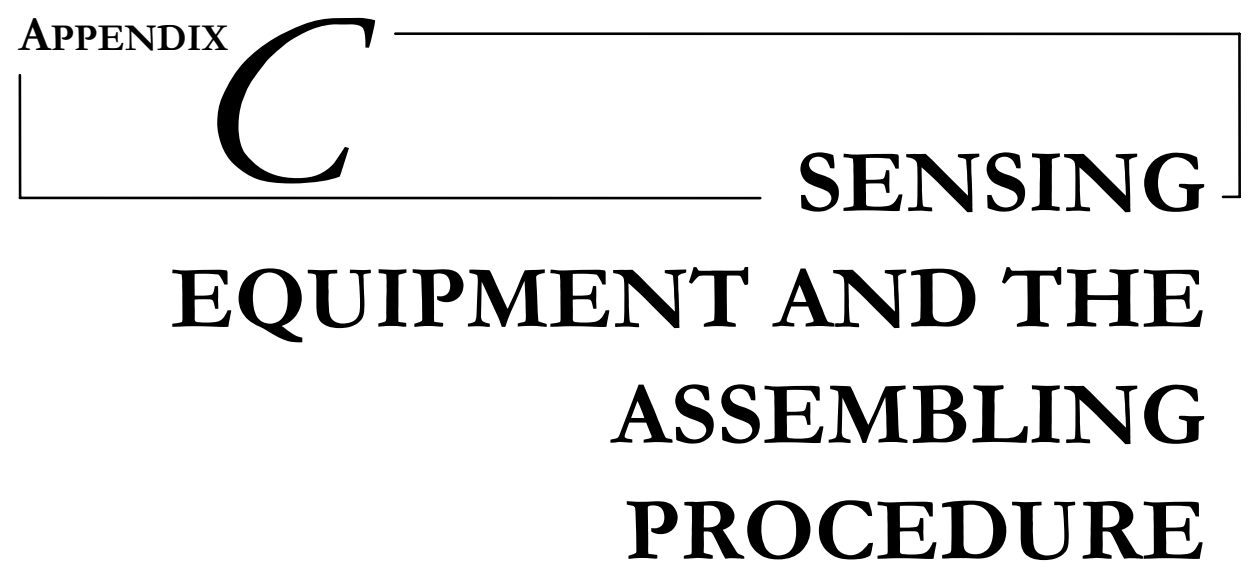

In Appendix $C$, a detailed overview of the sensing equipment, installed for the needs of this project in the experimental setup developed at the University of Twente, is given. The assembling procedure for the compressing section of the machine, and the helium charging procedure are described in the second part of it.

\section{C.I Sensing Equipment}

Measurements that were so far regarded in this thesis and related to the numerical simulations presented in Chapter 4, are the measurements of the gas pressure in the compressed section and buffer space, the compressed gas temperature, temperature of the inner surface of the cylinder head, temperature of the outer surface of the cylinder walls and the temperature of the piston. The measuring procedure, calibration, data processing and accuracy of the acquired data were earlier discussed in Chapter 2. In this Appendix we will make a larger overview of the utilized equipment itself, with specifications. 


\section{Pressure Transducers in the Compressed Space}

Pressure of the compressed gas was continuously measured with two pressure sensors. One is a Kistler Type 6052C3I piezoelectric pressure transducer, featuring very small dimensions and typically in use in internal combustion engines. The sensor uses a piezoelectric crystal which achieves high sensitivity $(\sim-20 \mathrm{pC} /$ bar $)$ that varies by not more than $\pm \mathrm{I}, \mathrm{O} \%$ in the operating temperature range, and a pressure range of $\mathrm{O}$ to $250 \mathrm{bar}$. It is installed in a $\mathrm{M}_{5} \mathrm{xO}, 5$ bore. The front seal allows very good heat dissipation and briefly a maximum operating temperature of up to $400{ }^{\circ} \mathrm{C}$. The passive acceleration compensation patented by Kistler keeps the influence of machine vibrations to a minimum. Linearity in all ranges is less than $\pm 0,4 \%$ FSO (Full Scale Output). This sensor was chosen for its allround design, extremely wide pressure range and robustness to temperature variations, vibrations and shocks, high sensitivity and very small dimensions. Nevertheless, an intrinsic property of the piezoelectric equipment is the charge drift during the measurement, and even though the sensor was coupled with an amplifier with a drift compensation feature (Kistler 5OIIBY50), it was not effective for the range and profile of experiments performed with a gas spring. This made the direct absolute measurements of the gas pressure impossible with only this sensor, and only the relative change, thus the relative pressure oscillation and the compression curve could be captured. For this reason, another more conventional pressure sensor was installed.

The second transducer mounted in the compressed space is a Kulite HKM-375M miniature pressure sensor with a metal diaphragma designed flush with the sensing surface and a piezoresistive sensing element located immediately behind this metal diaphragm which is protected by a metal screen. Force transfer is accomplished via a film of noncompressible silicone oil. This sensing sub-assembly is welded to a stainless steel body. The sensor features combined non-linearity, hysteresis and repeatability of \pm O.I $\%$ FSO BFSL (Best Fit Straight Line) in the operating temperature range of $-55^{\circ} \mathrm{C}$ to $+\mathrm{I} 75{ }^{\circ} \mathrm{C}$.

The two transducers were simultaneously used. Kistler piezoelectric sensor showed much smaller signal noise and thus provided better measurements, but the Kulite transducer was necessary to provide measurements of the absolute reference pressures and as a control measurement.

\footnotetext{
a It should be noted here that care had to be taken to meet the compatibility of the installed equipment the medium it was exposed to - helium. Namely, helium is extremely permeable and can pass the unprotected porous silicone membranes, damage them and also create a back pressure for the sealing membrane, making the measurements inaccurate. For this reason all the chosen pressure sensing equipment featured metal shielding to protect the sensing elements.
} 


\section{Pressure Transducers in the Buffer and Crankcase Space}

Transient measurements of the gas pressure in the buffer space were necessary in order to capture the leakage of the gas past the piston during the experiments and the oscillation in the 'counter' pressure on the other side of the compressing piston. This data was then used to provide the pressure profile and mass flux in the modelled mass source (sink) at the edge of the piston in the simulations. The utilized pressure transducer is a Druck PMP4070 displacement pressure sensor, with very high accuracy ( $\pm 0.08 \%$ FSO BFSL combined non-linearity, hysteresis and repeatability). The sensing silicon element is mounted within a high integrity glass-to-metal seal and is fully isolated from the pressure media by a Hastelloy isolation diaphragm.

Pressure measurements in the crankcase volume were of use during the vacuumizing and filling of the experimental setup with helium, as it was necessary to secure that all air from the setup was evacuated prior to the experiments. The installed sensor was an industrial Druck PMPI 400 sensor, with a typical accuracy of \pm O.I $5 \%$ and a stainless steel isolation shield protecting the micro-machined silicon pressure diaphragm.

\section{Gas Thermocouples}

Thermocouples measuring the compressed gas temperature were shortly described in Chapter 2. They are produced by Paul Beckman Company, Inc., Huntingdon Valley, USA. Sensors belong to the 300-A-x-O3-E series; E-type thermocouples (chromel-constantan) with a patented Micro-Disc ${ }^{\mathrm{TM}}$ hot junction $(<8 \mu \mathrm{m}$ thick). They are produced for the needs of this setup in two lengths: $25 \mathrm{~mm}$ and $6.5 \mathrm{~mm}$. The longer thermocouple was chosen to measure the temperature of the core gas, and the shorter $(6.5 \mathrm{~mm}$ was the smallest producible length) to measure the temperature of the gas in the vicinity of the wall (the boundary layer). As explained in Chapter 2, regardless of the extremely small thermal mass of the sensing end, dynamic gas temperature was not directly captured and a postprocessing algorithm had to be developed in order to use the recorded signal.

\section{Surface Temperature Sensors}

The cylinder top surface temperature sensors were also elaborated on in Chapter 2. They were initially conceptualized as heat flux sensors, having the surface and in-wall back thermocouples on a defined distance (0.5 $\mathrm{mm}$ in the wall). Nevertheless, as explained, the method for directly evaluating the heat transfer from these two temperature measurements was not developed during the course of this thesis, and the surface temperatures were used as the input for the numerical simulations, while the in-wall measurements were just an added indicator for the steady-state operation during the experiments. Construction of the surface sensor is such that a ribbon of chromel and a ribbon of constantan (E-type thermocouple), each $0.025 \mathrm{~mm}$ thick, are embedded in a copper probe body and separated 
by a sheet of mica $0.005 \mathrm{~mm}$ thick. The thermocouple junctions are formed by sanding the surface of the probe, which creates many microscopic thermocouple junctions. The back thermocouple is standard ( 2 wires and a junction) E-type thermocouple. The producer of these custom-made transducers is NANMAC Coorporation, Framingham, USA.

Surface thermocouples installed on the outer cylinder wall and cylinder top surfaces were off-the-shelf self adhesive thermocouples, type Omega SAI type T.

Infra-red temperature sensing of the outer wall surface was also attempted with a shortrange pyrometer (Raytek MI series), but this method did not bring any advantages compared to the thermocouple usage (resolution no better than $\mathrm{I}^{\circ} \mathrm{C}$, necessity to paint the surfaces or calibrate for reflectivity) and was retired.

Piston thermocouples are also mentioned in Chapter 2. Thermocouples are Thermo Electric MTS series, and the conductive resin is Arctic Silver 5.

\section{Crank Angle Encoder}

Crank angle encoder is made by Leine\&Linde, Type RHI 503. The sensor is mounted on the free end of the electric motor shaft, via a custom-made transition steel piece. The sensor has resolution of $3605 \mathrm{~V}$ TTL pulses per revolution and a zero-pulse.

\section{Data Acquisition System}

Data Acquisition System is thoroughly described in Chapter 2; here the summary of the features will be given:

- I x NI PCI-6250: I6 single ended/8 differential AI Channels; 24 Digital I/O Channels; ADC resolution: I6 bits; Input ranges: \pm IO V $\pm 5 \mathrm{~V}, \pm 2 \mathrm{~V}, \pm \mathrm{I} \mathrm{V}, \pm 0.5 \mathrm{~V}, \pm 0.2 \mathrm{~V}, \pm$ O.I V; Sampling Rate: I.25 MS/s single channel; I.OO MS/s multi-channel (aggregate); Absolute Accuracy for \pm O.I $\mathrm{V}$ range: $52 \mu \mathrm{V}$.

- I x NI PCI-6280: I6 single ended/8 differential AI Channels; 24 Digital I/O Channels; ADC resolution: I 8 bits; Sampling Rate: $625 \mathrm{kS} / \mathrm{s}$ single channel, $500 \mathrm{kS} / \mathrm{s}$ multi-channel (aggregate); Absolute Accuracy for \pm O.I V range: $28 \mu \mathrm{V}$; Programmable $40 \mathrm{kHz}$ low-pass filter.

- 2 x SCB-68 Shielded Terminal Blocks: I6 single ended/8 differential AI Channels; 2 AO

Channels; 8 DI/O Channels; IO PFI Lines; 2 x 5V Charge Lines;

- Shielded cabling. 


\section{Logging Computer}

The logging computer was optimized for high-speed sampling. Of importance here is a wide Front-Side-Bus on the processor for high data throughput, sufficient RAM memory and a fast hard disk. Selected computer thus featured an Intel ${ }^{\circledR}$ Core Duo Processor E6550 (2.33 GHz, I.333 MHz FSB, 4 MB L2 cache), 4,o GB $800 \mathrm{MHz}$ DDR2 RAM memory and a $250 \mathrm{~GB} 7200 \mathrm{rpm} 3.0 \mathrm{~Gb} / \mathrm{s}$ SATA2 hard drive.

\section{Filters}

Gas filters were utilized in the helium installation in order to eliminate any particles or humidity that would hamper the ideal gas approximation for the observed experiments. Three Walker Filtration A2O filter elements were used for this purpose: one Grade XI for I $\mu \mathrm{m}$, one Grade XA for the 0.OI $\mu \mathrm{m}$ debris filtration, and one Grade AC 0.OI $\mu \mathrm{m}$ active coal element for elimination of any possible moisture in the gas and/or the gas installation.

Another Grade $\mathrm{X}_{5}$ filter is installed in the cooling air installation, but, as described, cooling was not utilized in the experiments performed up to now.

\section{C.2 Assembling Procedure}

As described, the motoring section, comprising of the crankshaft mechanism and the lower piston, the crankcase, oil pump (for the lower piston) and charge and bleed valves, was acquired from Stirling Cryogenics \& Refrigeration B.V. It was not reconstructed in any way and for this reason it won't be described here; all the work was concentrated on the compressing section placed on top of it and in this subchapter will describe the assembling procedure of it.

First of all, all the parts, including tooling and the exposed surface of the lower piston have to be thoroughly cleaned prior to the installation. This includes degreasing with isopropanol, careful removal of any residual metal and dust particles with respect to very low tolerances in the sliding contacts, and working with latex gloves. After this, the assembling starts by placing the connecting-rod mounting plates on each of the two telescopic parts of the connecting rod, centring the pre-bored transversal holes in the two parts, placing a safety pin (Figure 2.2) and tight-pressing it (by centre-pinning). Connecting rod is then mounted on the compressing piston by placing a rubber O-ring $(3,4 \mathrm{xI}, 9)$ in the centre groove of the compressing piston seat and bolting it through the upper mounting plate. Next step is placing the free end of the rod (now with the compressing piston on the other end) in the seat of the lower piston (with another intermediate O-ring) and bolting it through the second mounting plate. The main frame is then carefully placed over the rod 
and the compressing piston and bolted to the base compressor. After this the cylinder liner is centred and slided over the piston and in the main frame until the outer rim of the liner sits on the inner rim of the main frame. Due to the very low tolerance between the compressing piston and the liner (33 $\mu \mathrm{m}$ in diameter), it is of extreme importance to very carefully position the liner with respect to the piston, in order to avoid clutching of two parts and/or plastic deformation (bending or fracture) in the flexible necks of the connecting rod. Prior to this, a rubber O-ring $(67.95 \times 2.62)$ is placed in the inner groove of the main frame, so to secure no leakage of the cooling air to the helium volume.

When the cylinder liner is fitted, a rubber O-ring (II0.72x3.53) is placed in the outer groove of the main frame, and a metal C-ring $(\varnothing 2.39,27.5 \mathrm{~mm}$ O.D.) in the inner groove of the liner. Another rubber O-ring (60.05xI.78) is placed in the bottom groove of the cylinder head, and then the head is carefully laid on the liner. After this, two disc-springs are placed on top of the cylinder head, and the top frame is centred, placed and bolted to the main frame. Feed-through plug with the helium capillary port is then fixed to the top of the top frame, and capillary further connected to the helium charge line.

During the entire mounting procedure, care has to be taken to properly route the lead wires from the sensing equipment. Before the main frame is placed, wires from the piston thermocouples need to be routed through the 3 wire ports in the wall of the frame and sealed with convenient feed-through elements. Before the top frame can be placed and bolted, all wires stemming from the gas thermocouples, cylinder wall and top outer surface thermocouples, heat flux sensor and the pressure transducer need to be guided out through the cooling inlet and the ports in the feed-through plug.

\section{Helium charging procedure}

After the machine is closed, helium lines connected and all lead wires screwed in the terminal blocks of the data acquisition system, the data monitoring software is started and the helium charging procedure can begin. A vacuum pump is connected to the filling line via a three-way valve (Figure 2.4), and the setup is vacuumized to an achievable underpressure (O.I-O.2 bar). Machine is then charged with helium to an overpressure of 5-6 bar, opening in sequence the capillary line, buffer space and crank case lines. Helium is then discharged (through the bleed valve to $\sim 2$ bar) and again vacuumized, and this sequence is repeated 3 times. Setup is then charged to the initial operating pressure and experiments can be performed. 


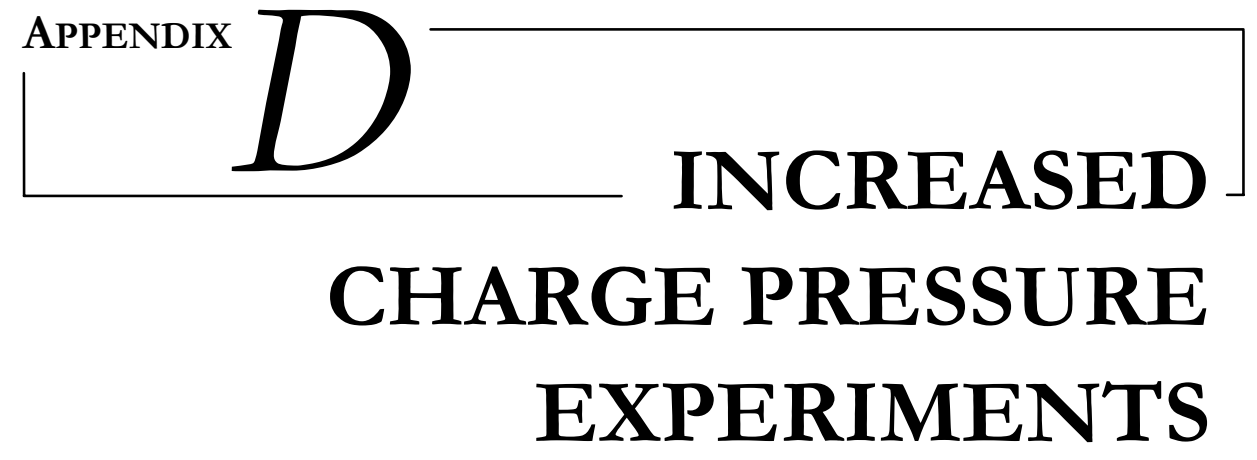

This Appendix presents the results of experiments featuring two bigher (than baseline) initial belium charge pressures. Results are plotted against the respective numerical simulations.

This section is an appendix of Chapter 4, with purpose of completing the report on the performed experiments and related simulations. The experimental setup was charged to absolute pressures higher than the baseline ( $\sim$ I.5 bar) and then the machine was operated. As explained in Chapter 4, the pressure that the setup is initially charged to, while at rest, does not equal the steady-state pressures at BDC that are measured and translated to the initial parameters for the numerical simulations. This is in relation to the displacement of the gas during continuous operation, which will typically result in the mean cycle pressure being close to the charge pressure before the start of the experiments, while the machine is at rest.

Results of these experiments are presented in Figure DI and D2, and show the sensitivity of the numerical models to the secondary motion of the piston and partial closing of the clearance gap between the compressing piston and the cylinder. Nevertheless, the error here does not exceed 0.38 bar, or $\sim 6.5 \%$ of the pressure amplitude. Experiments with higher charge pressures have not been performed for the compression ratio 8 , since it was considered that these would not have an added value to the results of this research at present stage. 

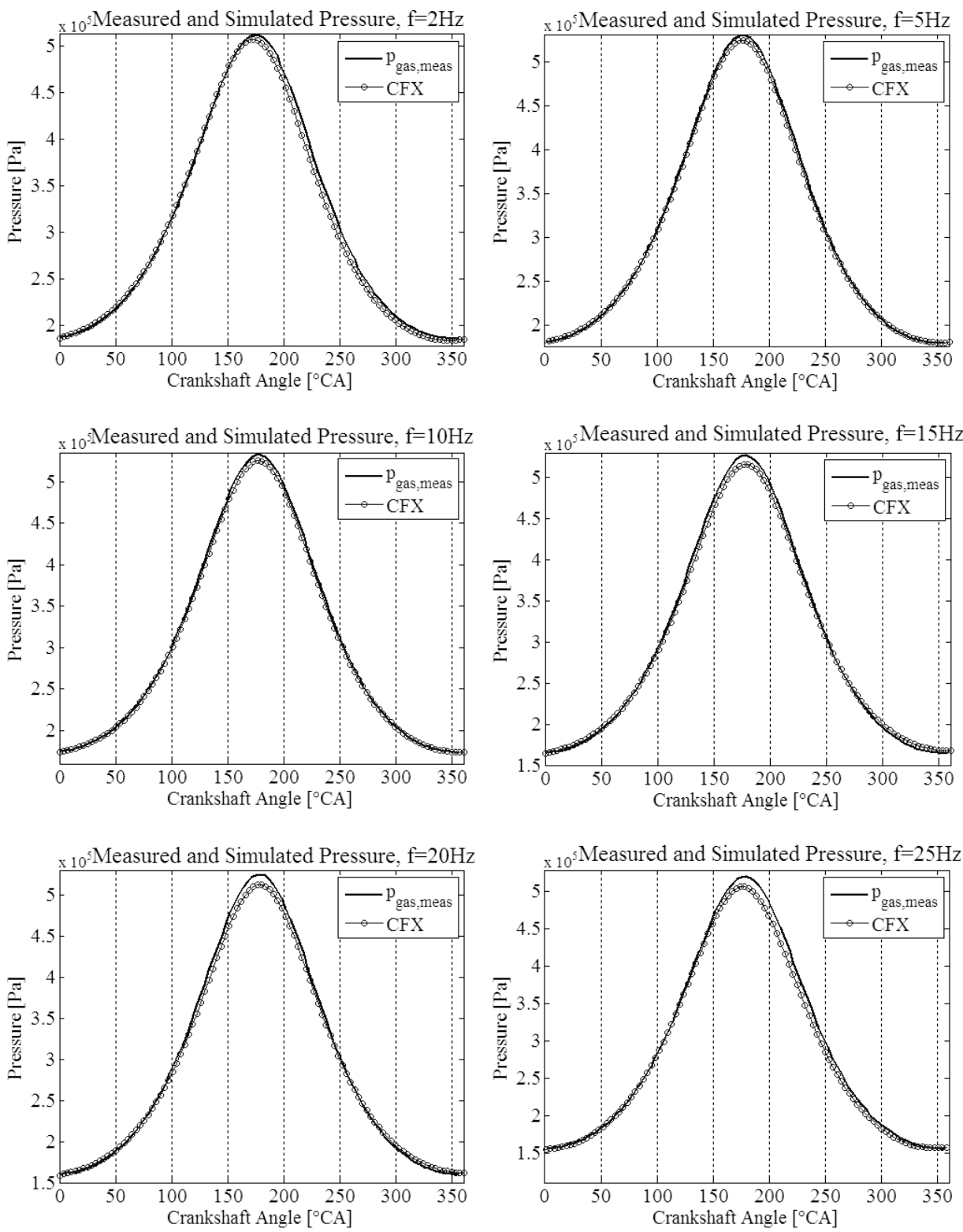

Figure D1: Experimentally measured and numerically simulated gas pressure with the modelled gas blow-by, $r=2$ UT setup, $p_{\text {charge }}=3$ bar 

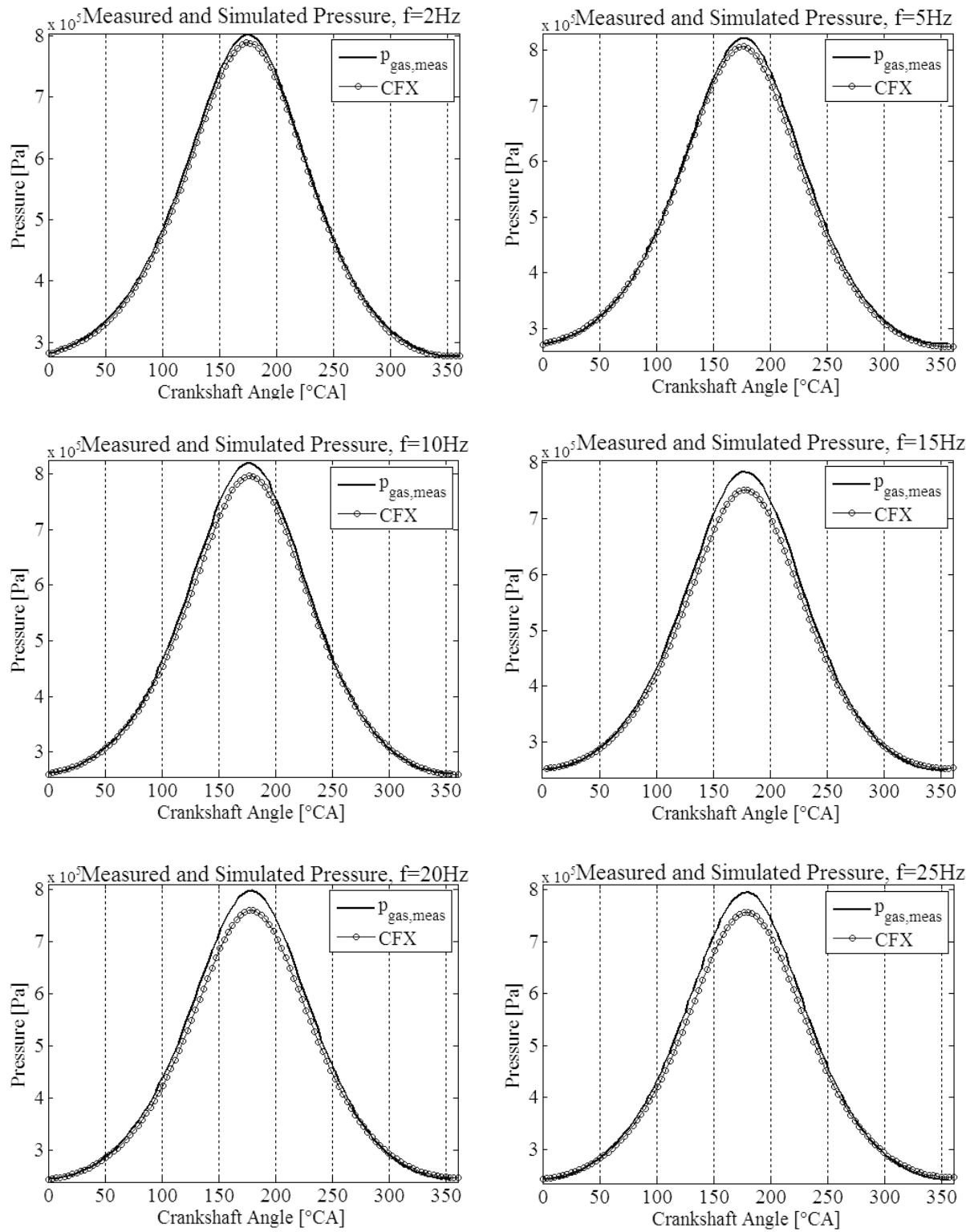

Figure D2: Experimentally measured and numerically simulated gas pressure with the modelled gas blow-by, $r=2$ UT setup, $p_{\text {charge }}=5$ bar 



\section{FINAL WORDS}

...And so we come to the end of it. Yes, the End. Let me ...summarize.

If one would ask me now: was I planning this when I was but a boy, to be doing research on thermodynamics and flows, dreaming to become a doctor of science? Mmm... honestly - no. Could I guess I would spend my twenties away from home, someplace I never knew before? Nope, I do not think so. Did I know what comes next? Well, once more, no. Was it long and difficult? Oohh... yes it was. But was it fun after all? ...Did I learn? Did I meet good people? Did I travel around? Do I have tales to tell? Am I happier? Yes, oh yes I am, and as I write this there is a big smile on my face to witness it.

In July-August 2002, I did my internship assisting the $\mathrm{PhD}$ project and under daily supervision of (now dr. ir.) Jaap van Kampen, at the very University of Twente and the Laboratory of Thermal Engineering. While shaking hands for goodbye, Jaap's mentor Jim Kok and the chair of department Theo van der Meer casually mentioned that I should return if I should ever consider postgraduate studies. We kept the contact alive and three years later I was behind my desk at the same department gripping on the heat transfer relations. You will thus be the first I will mention here: Jaap, thank you for all the assistance during that internship and the subsequent conversations that lead to my $\mathrm{PhD}$ position. Jim and Theo, many many thanks for the given chance, thank you for your guidance and sharing your knowledge and ideas with me, for your support and faith in me till the very end. I am very happy to walk out as another graduate from your department.

Knowledge, ideas, support... multiplied by tons of fun, coffee talks, good pranks, barbeques, cart races, soccer and an occasional drink, is what made even the Dutch rainy days light up. Artur, thank you for all the conversations and support when I needed it the most, and for agreeing to be my faithful paranymph next to Miladin. Timo!! my comrade, 
cheers for the brilliant office decorations, humour and the always bright sprit. Jan, Maarten, Ashiq, Marc, Rob, Anton, Marcel, Michiel, Sjoerd, Eddy... thanks guys, for your friendship and assistance along the way. Sally, for your help all the times when it was irreplaceable. Henk-Jan, Willie, Chris and Eise for all the technical support and help in the lab. Paweł, for having you as a partner on the project, and all the good work, ideas and views you gave. Huub, for being a great master graduate, and working so hard with me.

Big thanks to the STW foundation that sponsored this project and dr. ir. Corine Meuleman as its program officer, all the industrial partners mentioned in Appendix A, for their continuous valuable support, and Wim and Ten Heggeler Machinefabriek who have done wonderful work on our test rig. I wish to express special gratitude to Dr. Phil Stopford from ANSYS CFX, for welcoming me in Milton Park during my visit and all the precious assistance with my numerical work.

The 'Eva Sterk' group: Denis, Jon, Domokos, Paul, Yana, thanks so much for being my faithful companions in walking through cultural shocks and being there for laughs and tears. Much appreciated.

Milton my brother, Tatinho superstar, 'El Greco' Dimitrios... you know those days could not have passed if you were not next to me. I will keep reminding you of that, for the long years to come.

Paragraphs below will be addressed to my family and friends at home.

...I namerno ste izdvojeni jer vi koji ste u njima zaslužujete posebnu knjigu, kamoli malo odvojene pažnje.

Mama, tata i Maki, Vi ste zaslužni što postojim, što sam se školovao, što sam rastao zdrav i na kraju što sam gde jesam. Kada je bilo lepo i manje dobro, u uspesima i porazima, kad sam pri vrhu i kada se tek penjem, i sada kada potpisujem ovu knjigu, tu ste da me čujete, da me čuvate, gurate, radujete se i propatite sa mnom. Bili ste i bićete zauvek. Zahvalnost i ljubav prema Vama kao nikome drugom nosim uvek sa sobom, znajte. Draga familijo u Beogradu, Crnoj Gori i Kanadi, hvala vam na podršci od početka.

Moji najbolji: Luka, Nikola, Lazo, Marko, Jovice, Dačo, samo ja znam koliko ste mi nedostajali. I koliko mi je značilo što znam da imam vas tamo negde kući, da mi se radujete i čekate me kada se vratim. Ne bih sada bio ovde da nije bilo tako. Uki, majstori surčinci, Žare, vas ne odvajam od ove grupe. Olja, pratila si me i ohrabrivala od klinačkih školskih dana pa sve do danas. I davala svoju pozitivnu bajkovitu notu, bila najbolja drugarica koja se može poželeti. Uvek ću držati fige za tebe. Miladine, hvala za drugarstvo od fakultetskih dana pa preko ovih postdiplomskih i sve do danas. Čuvaj mi drugaricu. Mila A, hvala ti što si krenula sa mnom na ovakav put i bila mi podrška kada je bilo najteže. Mila M, za oslonac i sve osmehe koje si mi izvukla svojim besprekornim humorom. 
Gorice, što si ti, što si moja, i što sam tvoj. Neka tako ostane. Ova strana je samo tvoja.

Yours truly, iskreno vaš,

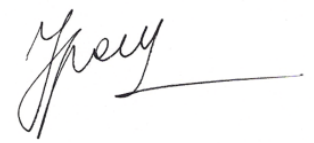

